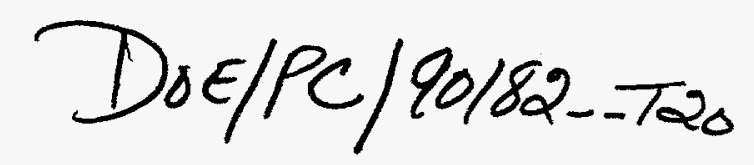

\title{
STUDIES OF GRANULAR FLOW DOWN AN INCLINED CHUTE
}

Work performed under PETC Contract \# DE-AC22-91PC90182

by

The University of Florida, Gainesville, Florida

Daniel M. Hanes, Principal Investigator

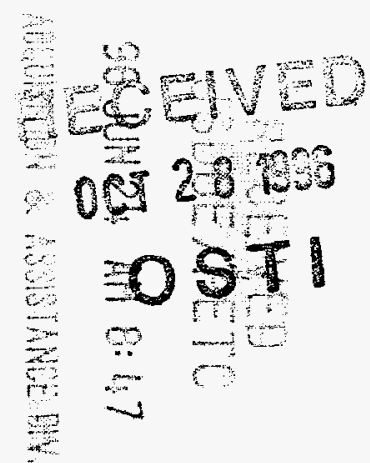

FINAL TECHNICAL REPORT

June, 1996

\begin{tabular}{|l|r|}
\hline Introduction & 2 \\
\hline Apparatus and Techniques & 3 \\
\hline Observations & 7 \\
\hline Conclusions & 160 \\
\hline References & 21 \\
\hline Appendix 1: Data Summary & $36 p$ \\
\hline Appendix 2: McCardle (1993) M.S. Thesis & $59 \mathrm{p}$ \\
\hline Appendix 3: Lee (1994) Ph.D. Thesis & $103 \mathrm{p}$ \\
\hline
\end{tabular}




\section{DISCLAIMER}

Portions of this document may be illegible in electronic image products. Images are produced from the best available original document. 


\section{STUDIES OF GRANULAR FLOW DOWN AN INCLINED CHUTE}

Work performed under PETC Contract \# DE-AC22-91PC90182

$$
\text { by }
$$

The University of Florida, Gainesville, Florida

Daniel M. Hanes, Principal Investigator

FINAL TECHNICAL REPORT

June, 1996

\begin{tabular}{|l|r|}
\hline Introduction & 2 \\
\hline Apparatus and Techniques & 3 \\
\hline Observations & 7 \\
\hline Conclusions & 16 \\
\hline References & 21 \\
\hline Appendix 1: Data Summary & $36 \mathrm{p}$ \\
\hline Appendix 2: McCardle (1993) M.S. Thesis & $59 \mathrm{p}$ \\
\hline Appendix 3: Lee (1994) Ph.D. Thesis & $103 \mathrm{p}$ \\
\hline
\end{tabular}




\section{Introduction}

When an assembly of discrete solid particles are placed upon an incline of sufficient slope they will move down-slope in any of a variety of possible flow regimes which generally exhibit different kinematics and dynamics. These regimes range from the uniform sliding motion of a bulk solid with little internal shear and essentially no particle velocity fluctuations, to a kinetic, granular flow with high internal shear and large particle velocity fluctuations. Flow regimes intermediate between these forms of motion are also possible, such as a layered flow with internal shear and small velocity fluctuations. The dynamics of any particular flow relate to the local mechanisms for momentum transfer and energy dissipation. These mechanisms are strongly influenced by the characteristics of the apparatus and its boundaries, such as the entrance conditions or the base friction and bumpiness. The connections between the apparatus characteristics and the local flow dynamics need to be determined in order to understand the basic behavior of the flow, as well as a variety of secondary phenomenon. For example, if segregation, mixing, or particle attrition are important in any particular application, then prediction and control of the flow regime would be of paramount importance.

We are therefore motivated to explore the flow regime behavior, and internal dynamics of a system of solid particles flowing down an incline. There have been many previous studies of this nature. These studies generally take one of two viewpoints in their general approach to this problem: 1) they emphasize the bulk flow behavior as it relates to the experimental apparatus, or 2) they emphasize the mechanics of the local flow behavior. From the first point of view, for example, Roberts (1969) or Ridgeway and Rump (1970) describe how a smooth base results in greater discharge by increasing the slip at the base. From the second point of view, Savage (1979) or Drake (1991) describe the relative importance of collisions and friction in determining the constitutive behavior of the observed shear flows. Some authors, such as Johnson, Nott, and Jackson (1990) discuss both viewpoints. They evaluated the constitutive behavior and its relation to the flow regime which was in turn related to the entrance conditions of the particular apparatus.

These previous investigations pose some challenging questions which provide the motivation for the present studies. For example, can any flows down an incline be accurately described by the kinetic theory for granular flow? Are any flows fully developed in space and steady in time? What is the significance of the interactions between the flow particles and the base? Are multiple flow regimes possible under similar conditions? In this work we will begin to answer some of these questions. In particular, we will provide measurements which address the issues of flow development and steadiness. We will also examine the effects of a bumpy base upon the local characteristics of the flow. 


\section{Apparatus and Techniques}

An inclined chute was constructed as shown in Figure 1. The flow region of the experimental apparatus consists of a rectangular aluminum chute with clear lexan sidewalls. The interior of the chute is four meters long, $46 \mathrm{~cm}$ tall, and the width is adjustable from zero to $25 \mathrm{~cm}$ in $2.5 \mathrm{~cm}$ increments. A drawing of the chute cross section appears in Figure 2. The $19 \mathrm{~mm}$ channel design of the chute base was designed to deflect less than $.25 \mathrm{~mm}$ for the entire chute when supported horizontally by its ends. The base of the chute is designed to be covered with interchangeable inserts, allowing different base characteristics to be studied.

The angular range which the chute is operable is $10^{\circ}$ to $30^{\circ}$. Additionally, the chute is adjustable to within the $0.25 \mathrm{~mm}$ tolerance at any angle. In order to achieve this, the chute is supported at six points and pivots at the top (entrance) in a single plane, as shown in Figure 3. The lower four supports consist of square telescoping tubing to allow for the various angles. At the base of each of these adjustable legs is a worm gear screwjack. One complete turn of the shaft on these jacks extends the leg 0.635 $\mathrm{mm}$. At the two top pivot points there are adjustable wedge jacks. These six jacks (one for each support point) allow the chute to have the micro-adjustability required. There are ten pivot points, each physically achieved by a pillow block bearing.

The driving force for the granular flow in the experimental region is gravity. The vehicle which re-circulates this flow is a $46 \mathrm{~cm}$ wide Corra-Trough belt conveyor manufactured by Buck-El, Inc. Entrance and exit chambers were designed to route the flow between the chute and the conveyor. Both devices had to be flexible because the position of the chute relative to the conveyor changes each time the angle of the chute is changed. A $15 \mathrm{~cm}$ iris valve controls the main flow between the top hopper and the entrance chamber.

An adjustable gate apparatus was constructed to accurately control the mass flux at the upper entrance of the chute. This mechanism, shown in Figure 4 positioned at the top of the chute, is adjustable for any width and can be positioned anywhere along the length of the chute. The aluminum plate which forms the gate itself is what allows the adjustability as it is completely interchangeable.

The first step in setting up the chute for a particular experiment is to set the angle of inclination. The chute is supported by a movable hoist and the pins are removed from the four telescopic leg supports. Pivoting about the two top support points, the chute is lowered to the proper angle. The angle is read by an Inogon inclinometer, which uses a vernier scale and two diffraction grating discs and is accurate to $0.2^{\circ}$. The pins are replaced and the hoist removed when the angle is close to the desired inclination. A somewhat iterative process is performed whereby an angle reading is taken at different points on the chute base and the six legs of the chute are adjusted until the angle is the same at all points. 


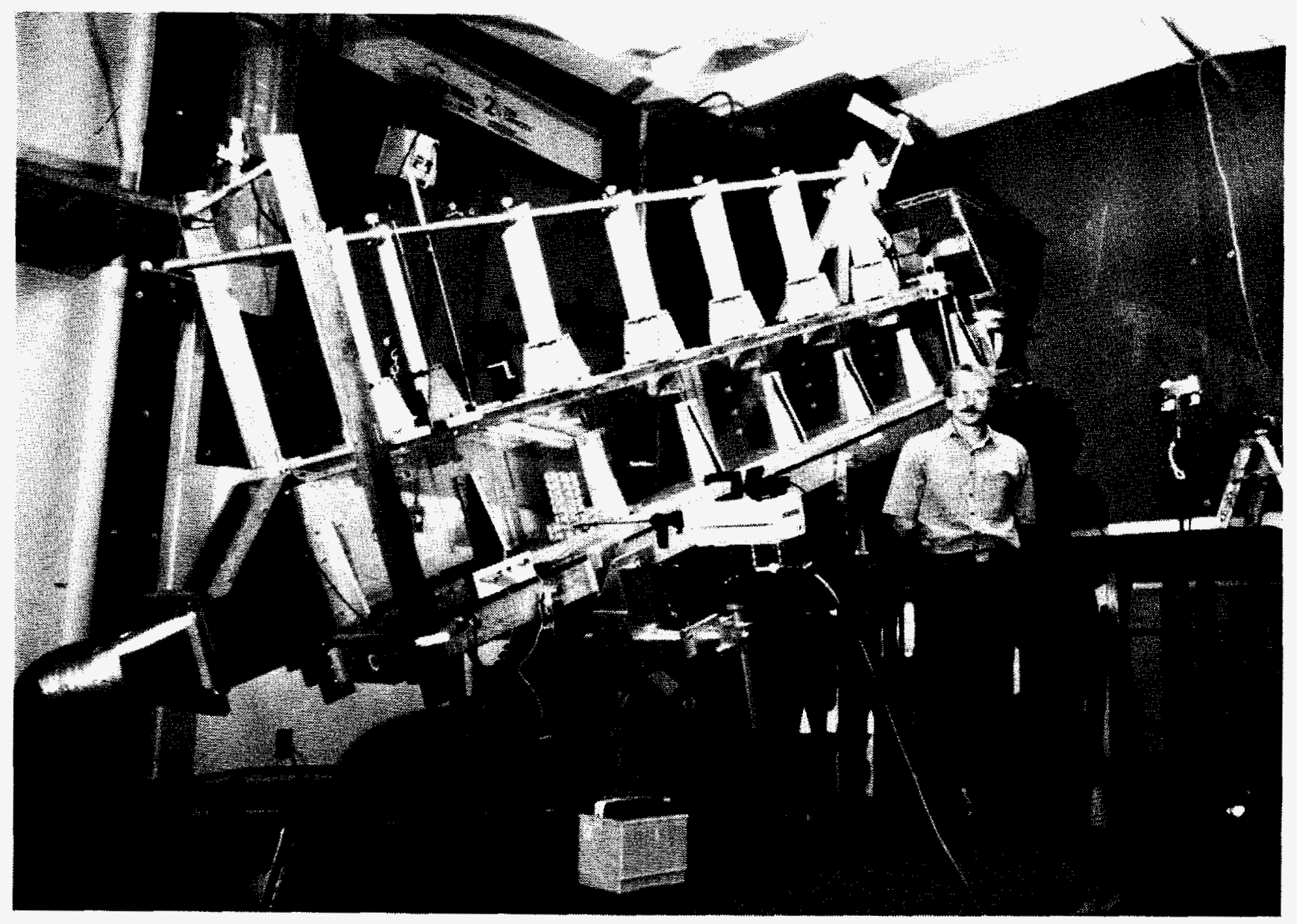

Fig. 1: Photograph of the inclined chute facility. 


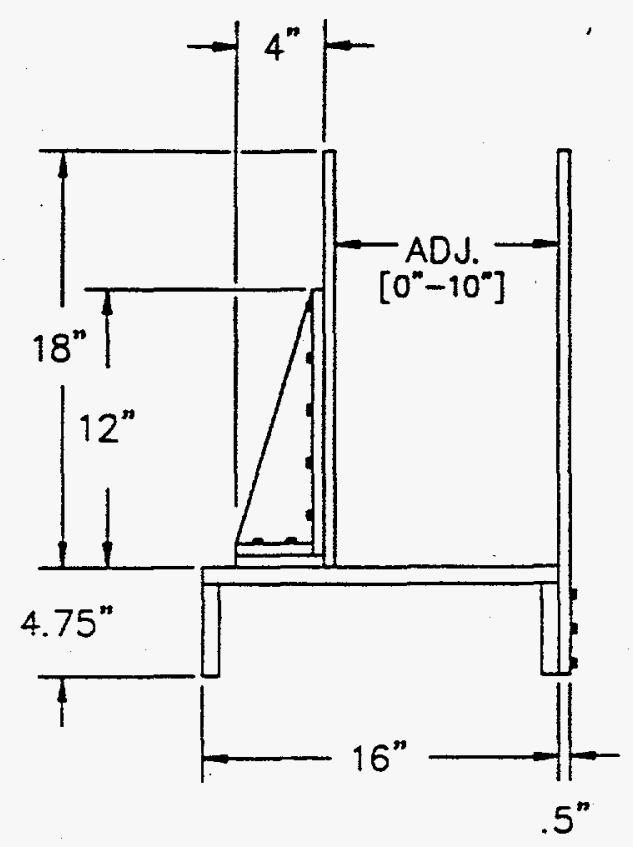

Fig. 2: Cross-section of chute.

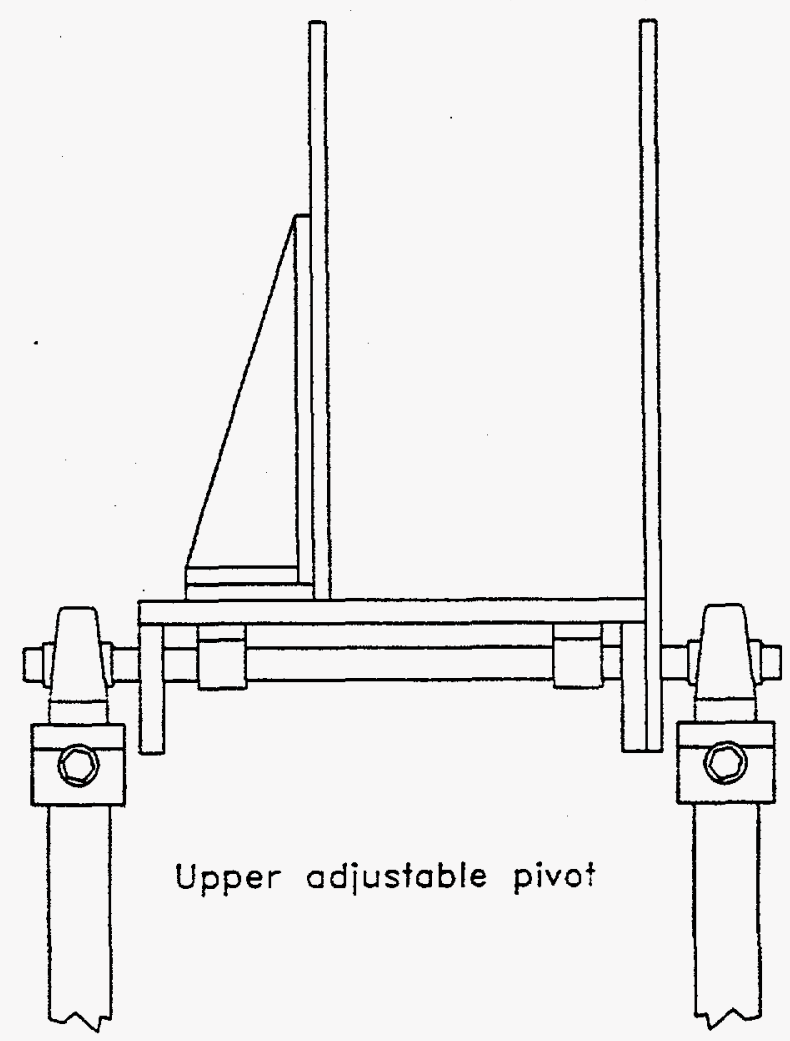

Fig. 3: Diagram of chute support member. 


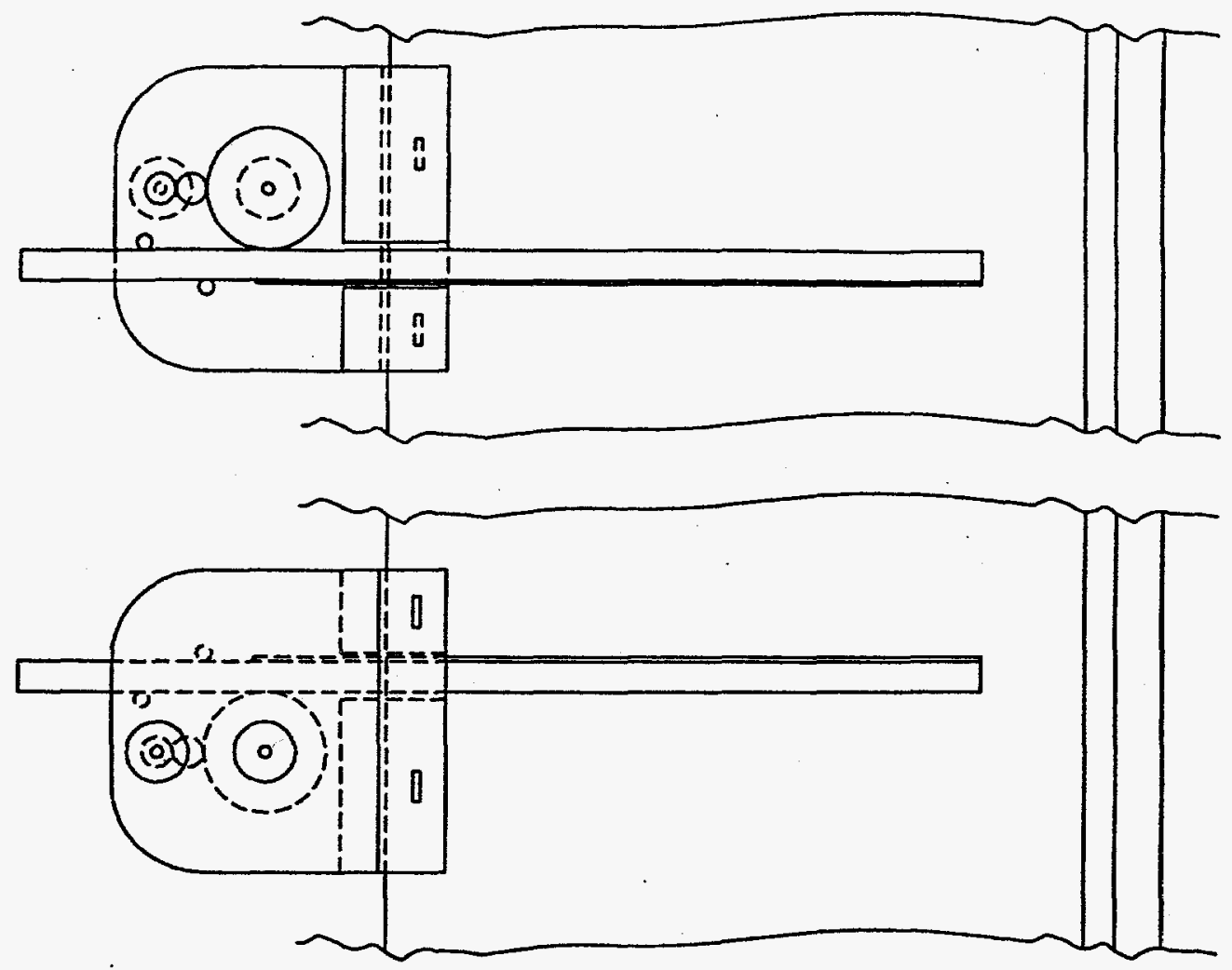

\begin{tabular}{l}
0 \\
0 \\
4 \\
\hline \\
0 \\
0
\end{tabular}

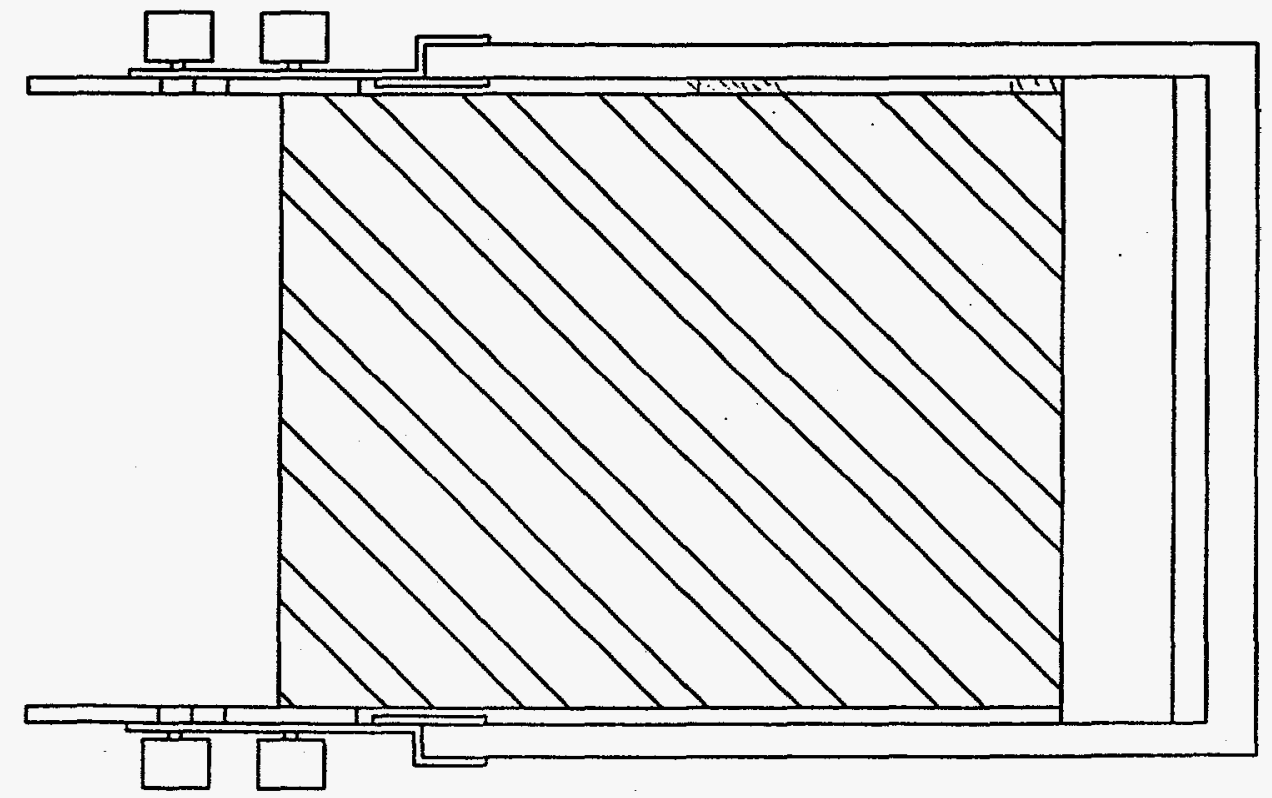

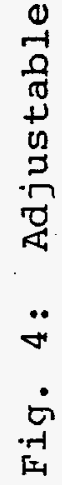


The granular material used in the experiments described in this report are technical quality glass spheres, three millimeters in diameter. These beads are produced by Cataphote, Inc. Cataphote lists the tolerances for the $3 \mathrm{~mm}$ spheres at $\pm 0.2 \mathrm{~mm}$. The average mass of a single bead was measured to be $0.034 \mathrm{~g}$ which gives an average measured specific gravity of the glass at $2.42 \mathrm{~g} / \mathrm{cm}^{3}$.

A variety of instruments and mechanical devices are utilized to obtain measurements of flow parameters. These consist of measurements of bulk properties of the flow as well as local properties. A summary of the range, precision, and accuracy of the measurements is given in Table 1.

Bulk flow measurements consist of the flow thickness and material mass in each of six sections along the chute, along with the total mass discharge rate. From these measurements we calculate bulk flow velocity, volume concentration, mass hold up, and thickness in each chute section.

Local diagnostics include fiber optic sensors and high speed digital imaging, generally in section 6 of the chute. These data were used to estimate the velocity profiles near the side wall and at the flow surface across the chute. The measurement techniques were developed during this project and are summarized in the theses of McCardle (1993) and Lee (1994), presented in Appendices 2 and 3, respectively. The different methods of determining velocity are compared to each other in Figure 5. The method referred to as manual particle tracking is labor intensive, and only used here to verify the accuracy of the automated fiber optic and high speed imaging techniques. In general, a DIV based correlation scheme was applied to generate velocity profiles from pairs of images. This technique proved reliable and accurate for most flow conditions.

\section{Observations}

A variety of experiments have been conducted in order to explore the issues presented earlier. A summary of these experiments is given in tabular form in Appendix 1. We will present results now in the following order: flow regime, examples of fully developed flow, effects of base bumpiness, and temporal fluctuations.

Different flow regimes can be achieved in the chute in a variety of ways. However, if the base, sidewalls, and the type of entrance/exit are kept the same, then the variables which can be varied are the slope and the entrance gate height. As an example of the flow regimes which are possible, we will present next some results for the flow of 3 $\mathrm{mm}$ glass spheres over a bumpy base. The base consists of $3 \mathrm{~mm}$ glass spheres rigidly fixed in a hexagonal pattern with $4 \mathrm{~mm}$ separation between bead centers.

When the slope is too low (less than approximately 16 degrees for the present application), then decelerating flow or no flow results. The decelerating flows are dense, slow, and layered, with very small velocity fluctuations. If the slope is high 
TABLE 1. Precision and accuracy of the measured, fixed, and calculated values associated with the chute.

\begin{tabular}{|c|c|c|c|c|c|}
\hline OHantis & Mpikal Values. & Rang & pression & Heringrym & "yrica! \\
\hline Flow Thickness & $3.3 \mathrm{~cm}$ & $0-30 \mathrm{~cm}$ & $0.1 \mathrm{~cm}$ & $0.1 \mathrm{~cm}$ & $3.03 \%$ \\
\hline $\begin{array}{c}\text { Mass in a Chute } \\
\text { Section } \\
\end{array}$ & $2340 \mathrm{~g}$ & $0-8000 \mathrm{~g}$ & $5.0 \mathrm{~g}$ & $10.0 \mathrm{~g}$ & $0.43 \%$ \\
\hline Discharged Mass & $4645 \mathrm{~g}$ & $0-8000 \mathrm{~g}$ & $5.0 \mathrm{~g}$ & $10.0 \mathrm{~g}$ & $0.22 \%$ \\
\hline Gate Height & $5.08 \mathrm{~cm}$ & $0-30 \mathrm{~cm}$ & $0.15 \mathrm{~cm}$ & $0.15 \mathrm{~cm}$ & $2.95 \%$ \\
\hline Humidity & $53 \%$ & $30-80 \%$ & $1 \%$ & $2 \%$ & $3.77 \%$ \\
\hline Air Temperature & $78 \mathrm{~F}$ & $60-90 \mathrm{~F}$ & $0.5 \mathrm{~F}$ & $1.0 \mathrm{~F}$ & $1.28 \%$ \\
\hline Chute Inclination & $\begin{array}{l}17.0 \mathrm{deg} \\
22.5 \mathrm{deg}\end{array}$ & $\begin{array}{c}0.00-19.99 \\
20.0-28.0\end{array}$ & $\begin{array}{c}0.01 \mathrm{deg} \\
0.1 \mathrm{deg}\end{array}$ & $\begin{array}{c}0.01 \mathrm{deg} \\
0.1 \mathrm{deg}\end{array}$ & $\begin{array}{l}0.06 \% \\
0.44 \%\end{array}$ \\
\hline $\begin{array}{c}\text { Mass of Beads in } \\
\text { System }\end{array}$ & $150 \mathrm{E} 3 \mathrm{~g}$ & $0-150 \mathrm{E} 3 \mathrm{~g}$ & $1 \mathrm{~g}$ & $4500 \mathrm{~g}$ & $3.00 \%$ \\
\hline Chute Bed Length & $365.8 \mathrm{~cm}$ & $365.8 \mathrm{~cm}$ & $0.15 \mathrm{~cm}$ & $0.15 \mathrm{~cm}$ & $0.04 \%$ \\
\hline Chute Bed Width & $15.24 \mathrm{~cm}$ & $15.24 \mathrm{~cm}$ & $0.15 \mathrm{~cm}$ & $0.15 \mathrm{~cm}$ & $0.98 \%$ \\
\hline Section Length & $50.2 \mathrm{~cm}$ & $50.2 \mathrm{~cm}$ & $0.15 \mathrm{~cm}$ & $0.30 \mathrm{~cm}$ & $0.60 \%$ \\
\hline Average Bulk Velocity & 3.9 & $0-10$ & & & $6.0 \%$ \\
\hline Mass Holdup & 4.0 & $0-10$ & & & $7.0 \%$ \\
\hline Volume Fraction & 0.38 & $0-.6$ & & . & $4.4 \%$ \\
\hline Mass Flow Rate & 15.8 & $0-30$ & & & $0.24 \%$ \\
\hline
\end{tabular}




\section{Particle Velocity Using Three Measurement Techniques}

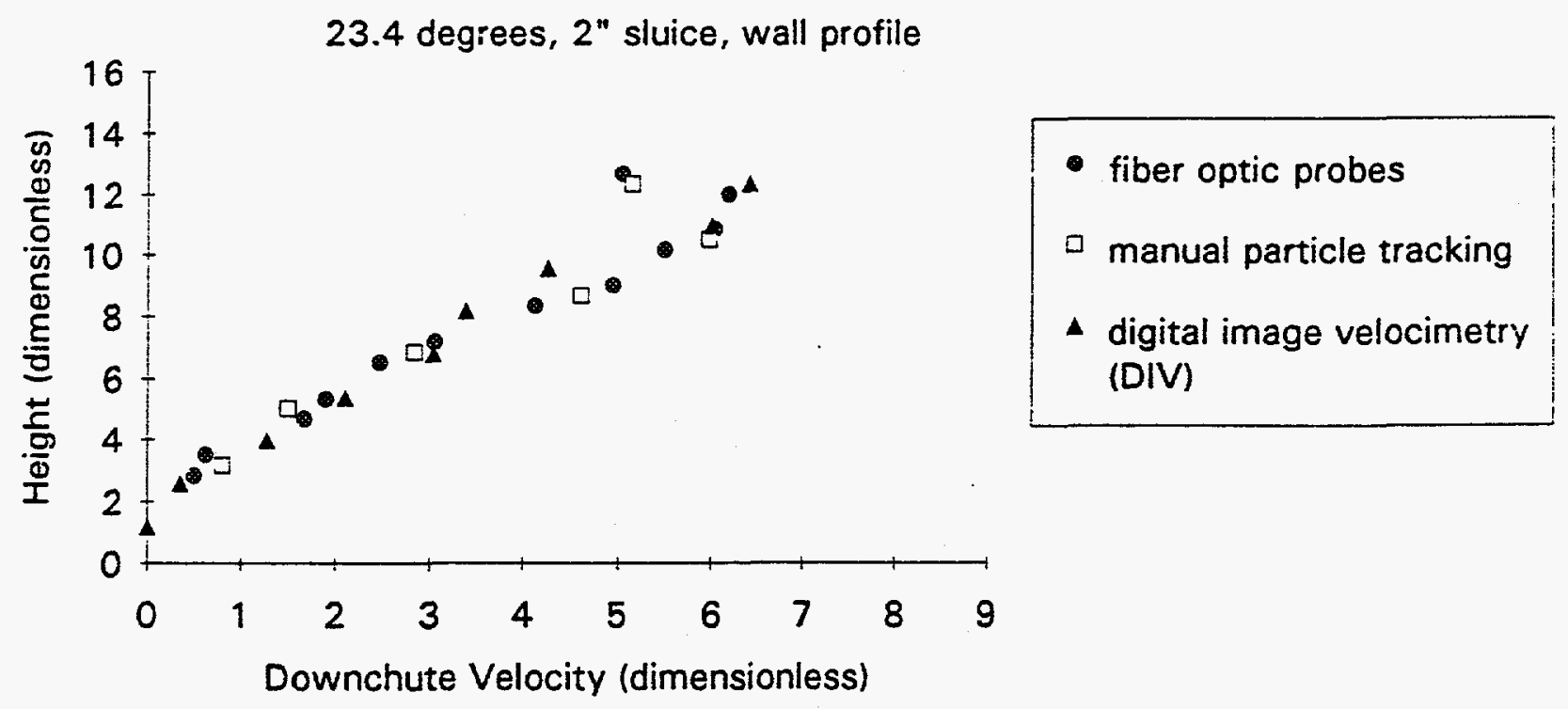

Fig. 5: Comparison of three methods used to measure velocity. 
(greater than approximately 24 degrees for the present application), then the flow accelerates and expands down-chute, exhibiting large velocity fluctuations and low volume concentration. In between these extremes, flows exhibit complex and varied behavior. We have classified these flows into several categories based upon their qualitative character. Surging flows are dense and slow, and intermittently surge like a wave. They resemble the decelerating flows described above. Sometimes between surges the material appears stationary. Frictional flows exhibit shear, layering, high volume concentration, and weak velocity fluctuations. Collisional flow exhibit shear, weak layering, intermediate volume concentration, and strong velocity fluctuations. The distinction ietween frictional and collisional flows is not always obvious; sometimes high speed imaging was used to delineate the flows based upon the length of contact between neighboring particles. Sometimes a flow was collisional throughout most of the depth, but frictional near the base. These flows will be discussed further in the section regarding the influence of the base. Finally, saltating flows are comprised of particles which bounce off the base and follow approximately ballistic trajectories. These flows are dilute, with rare interparticle collisions.

Fully developed flows were achieved in both the frictional and collisional regimes. For example, at a slope of 21.2 degrees, a fully developed frictional flow was observed. Figure 6 shows the flow depth, bulk volume fraction, and bulk velocity as a function of position in the chute. To within the accuracy of the measurements, the flow is fully developed. High speed images indicate a dense, layered flow, with enduring contacts between neighboring particles. The velocity profiles through the side wall and across the free surface (looking down on the flow) are shown in Figures 7 and 8. There is slip at both the base and the side walls, and shear throughout the flow. The slip at the bumpy base is much less than the slip at the sidewalls, and the corresponding shear in the base-normal direction is much greater than the shear in the base-parallel direction.

At a slope of 23.4 degrees, a fully developed collisional flow was achieved, as shown in Figure 9. High speed images indicate brief contact between neighboring particles, except at the base. There is enduring contact and small slip at the base, and large slip at the side walls. The velocity profiles at the side wall and across the free surface are shown in Figures 7 and 8. Again, the base-normal shear is much greater than the baseparallel shear. The measured and calculated data for 21.2 degrees and 23.4 degrees is summarized in Table 2.

The bumpy base, although composed of low friction glass spheres $(\mathrm{mu}=0.1)$, appears to nearly capture a layer of the flowing spheres. Even in the collisional flows, the lowest layer of flowing spheres moves intermittently, and remains in contact with the base much longer than the time required for a collision. In contrast to the kinetic theory for a bumpy base put forth by Hanes et. al (88), the base does not act as a source of fluctuation energy. Rather, the base acts as a sink for fluctuational energy, much like an internal erodible boundary. This can also be seen by examining the base- 
21.2 deg: 2" sluice

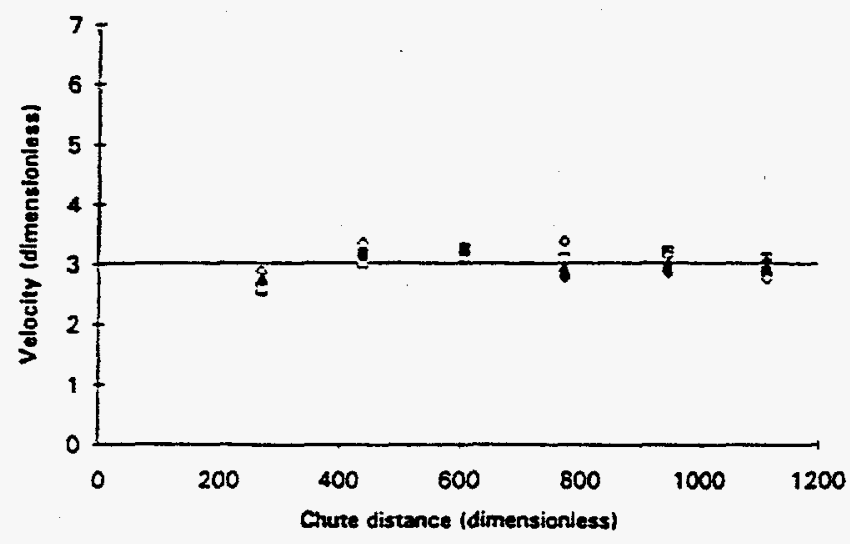

21.2 deg: 2" sluice

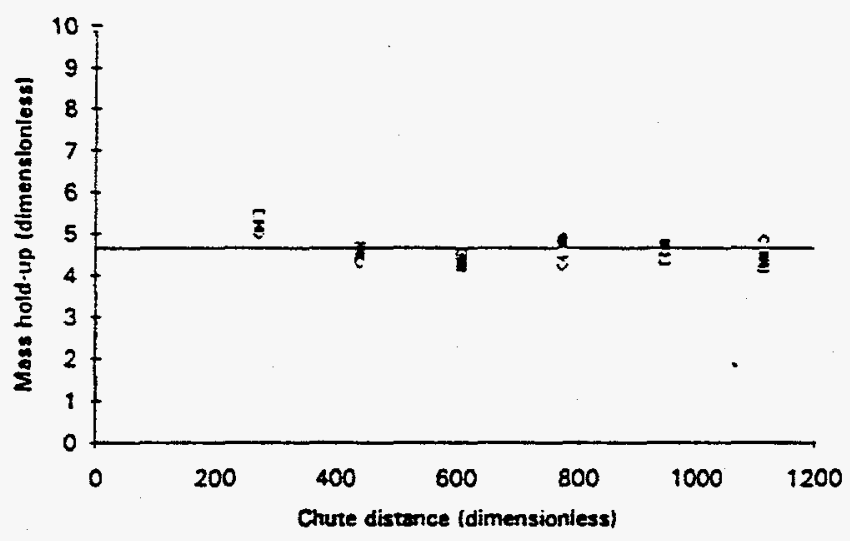

21.2 deg: $2^{n \prime}$ sluice

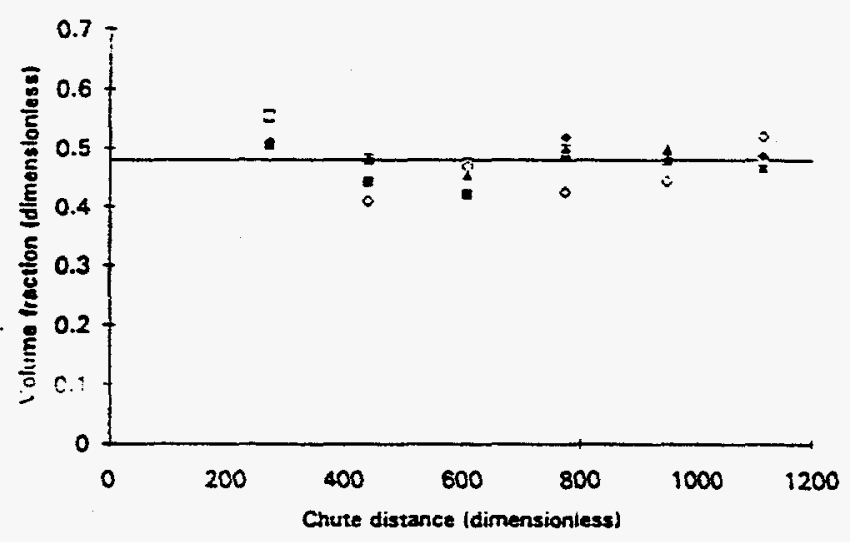

Fig. 6: Frictional flow at $21^{\circ}$. 


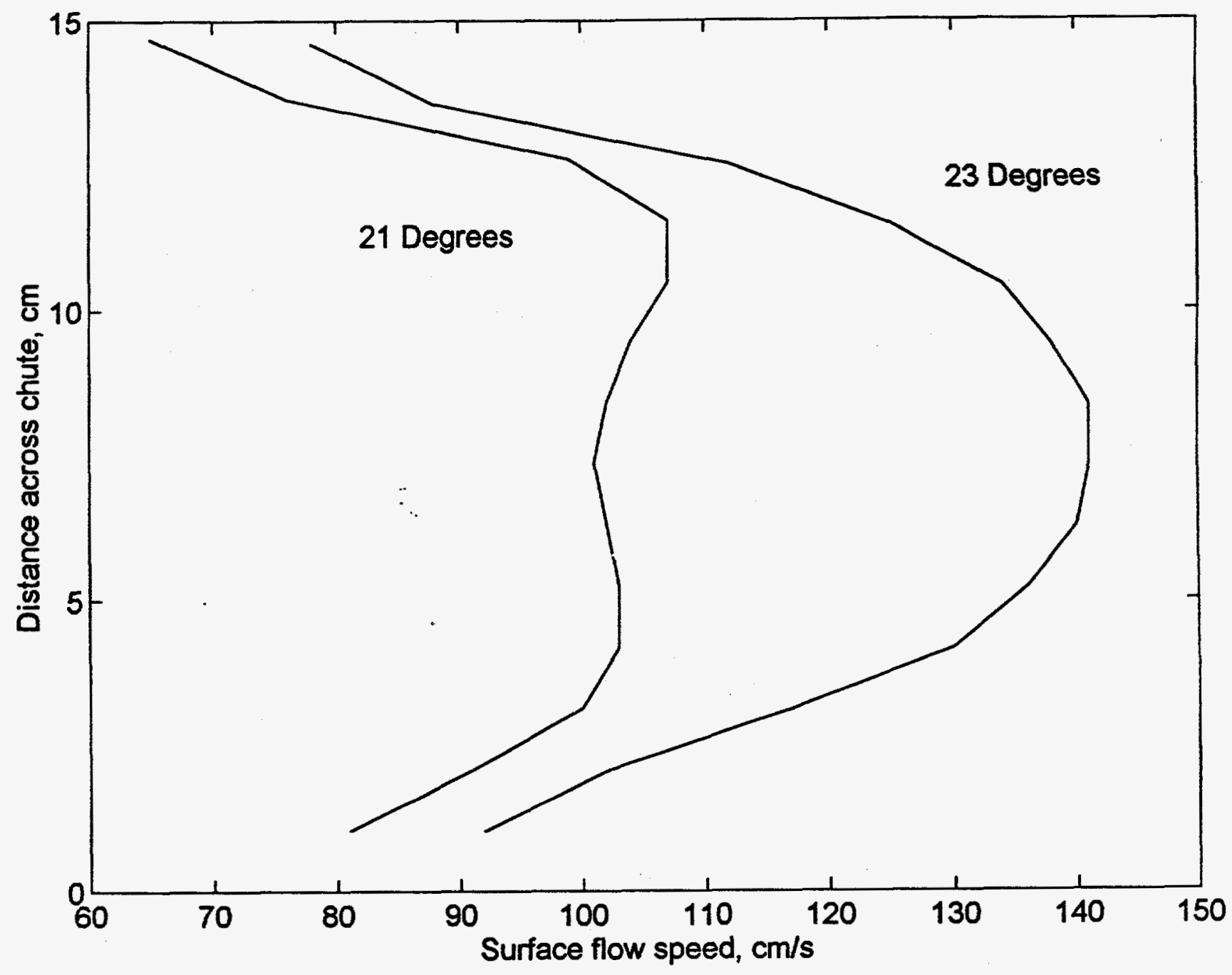

Fig. 7: Velocity profile at side wall for $21^{\circ}$ and $23^{\circ}$. 


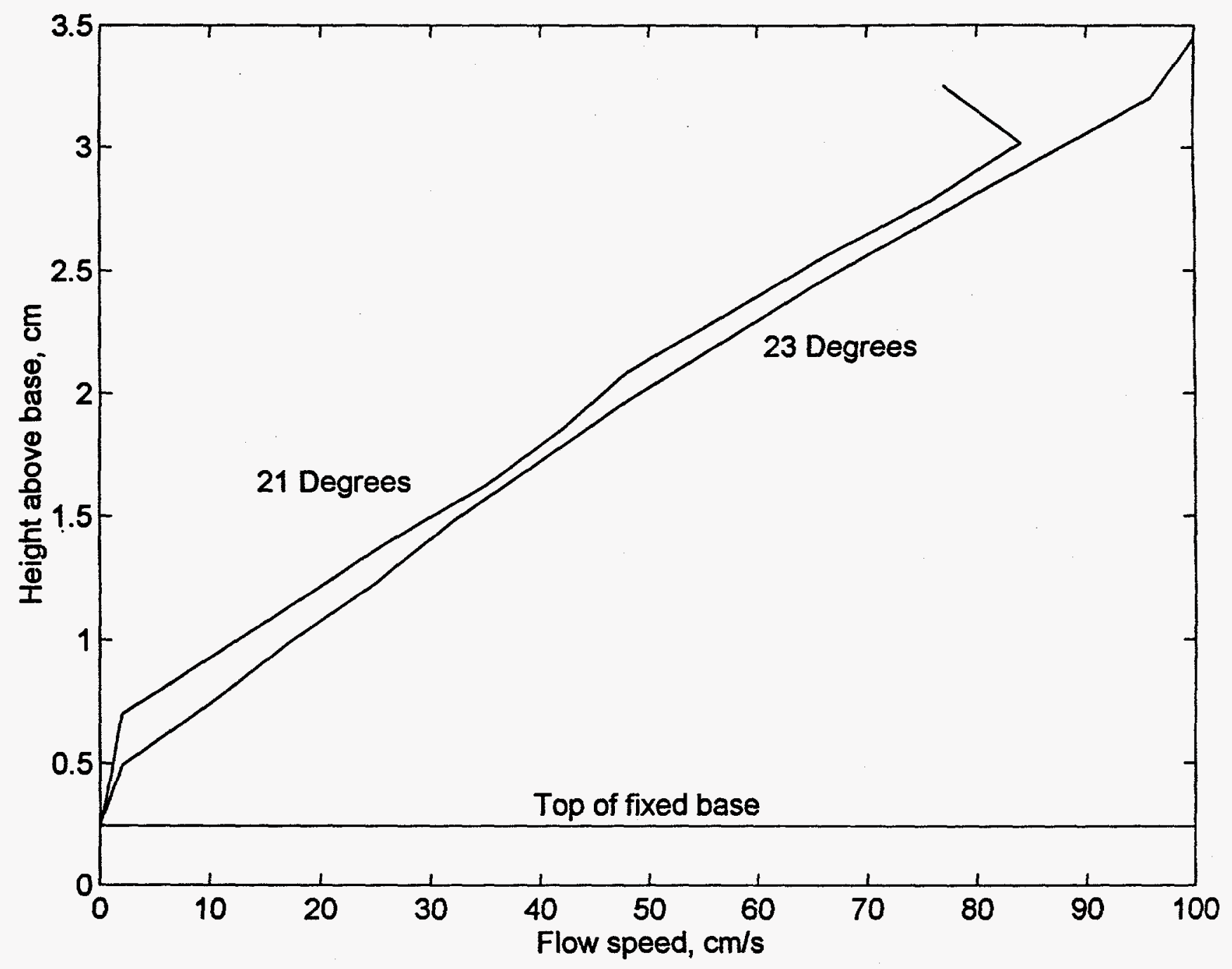

Fig. 8: Velocity profile at free surface for $21^{\circ}$ and $23^{\circ}$. 
23.4 deg: $2^{-}$sluice

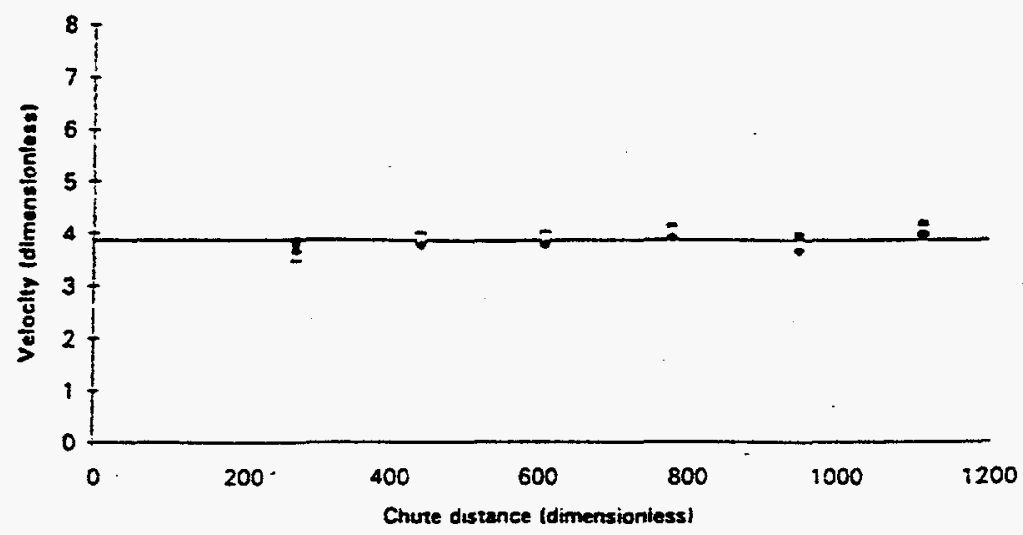

23.4 deg: 2" sluice

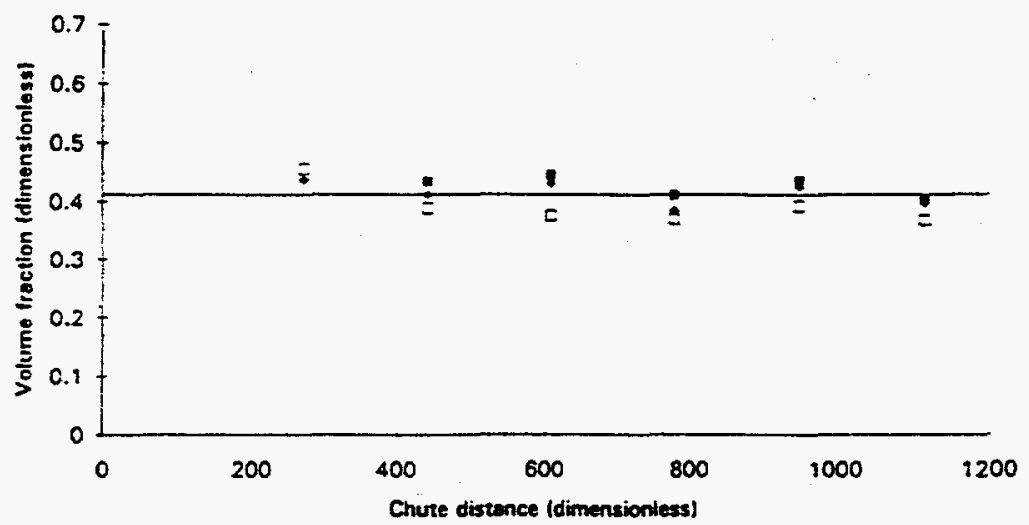

23.4 deg: $2 "$ sluice

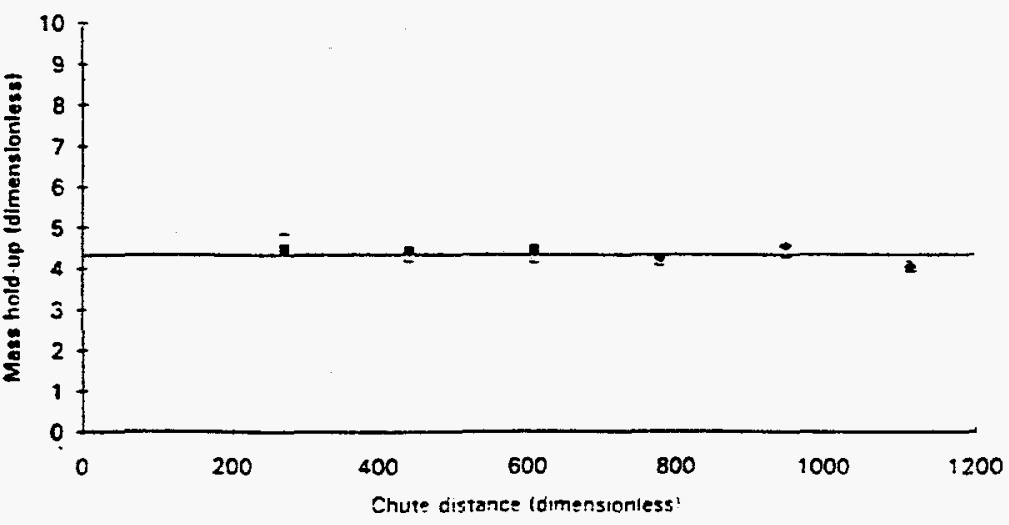

Fig. 9: Collisional flow at $23^{\circ}$. 
TABLE 2

\section{4 degrees, 2" sluice}

Values averaged over 3 runs, taken from section $6 *$ of the chute

$\begin{array}{llll} & & \text { dimensionless } & \text { factor } \\ \text { Flow thickness: } & 3.4 \mathrm{~cm} & 11.3 & \mathrm{~d} \\ \text { Discharge: } & 3.29 \mathrm{~kg} / \mathrm{sec} & 16.8 & \rho \mathrm{\rho dw}(\mathrm{gd}) .5 \\ \text { Solid Vol. fraction: } & 0.40 & 0.40 & 1 \\ \text { Mass hold-up: } & 3.3 \mathrm{e}-3 \mathrm{~kg} / \mathrm{cm}^{2} & 4.4 & \mathrm{\rho d} \\ \text { Velocity: } & 66 \mathrm{~cm} / \mathrm{sec} & 3.83 & (\mathrm{gd}) .5 \\ \text { Trapped mass: } & 2.67 \mathrm{~kg} & & \\ \text { Base area: } & 809.2 \mathrm{~cm}^{2} & & \\ \text { Base: hexagonal center to center }=0.4 \mathrm{~cm} & & \end{array}$

$$
\begin{array}{ll}
\text { Approx. } 94 \text { particles } / \mathrm{cm}^{2} & \mathrm{~d} \text { (particle diameter) }=0.3 \mathrm{~cm} \\
& \rho \text { (particle density) }=2.5 \mathrm{e}-3 \mathrm{~kg} / \mathrm{cm}^{3} \\
& \text { w(chute width) }=15.24 \mathrm{~cm} \\
& \mathrm{~g} \text { (gravity) }=9.81 \mathrm{~m} / \mathrm{s}^{2}
\end{array}
$$

\section{2 degrees, 2" sluice}

Values averaged over 5 runs, taken from section $6^{*}$ of the chute

$\begin{array}{llll} & & \text { dimensionless } & \text { factor } \\ \text { Flow thickness: } & 2.9 \mathrm{~cm} & 9.7 & \mathrm{~d} . \\ \text { Discharge: } & 2.76 \mathrm{~kg} / \mathrm{sec} & 14.1 & \rho \mathrm{\rho dw}(\mathrm{gd}) .5 \\ \text { Volume fraction: } & 0.48 & 0.48 & 1 \\ \text { Mass hold-up: } & 3.4 \mathrm{e}-3 \mathrm{~kg} / \mathrm{cm}^{2} & 4.6 & \rho \mathrm{\rho d} \\ \text { Velocity: } & 53 \mathrm{~cm} / \mathrm{sec} & 3.08 & (\mathrm{gd}) .5 \\ \text { Trapped mass: } & 2.79 \mathrm{~kg} & & \\ \text { Base area: } & 809.2 \mathrm{~cm}^{2} & & \\ \text { Base: hexagonal center to center }=0.4 \mathrm{~cm} & & \end{array}$

$$
\text { Approx. } 98 \text { particles } / \mathrm{cm}^{2}
$$

*section 6 is the second-to-last downchute section in which the boroscope and fiber optic sensors are used to make measurements. 
normal profile of the standard deviation of velocity, as shown in Figure 10. The fluctuations clearly decrease toward the base, and increase toward the free surface.

Experiments were repeated for a slightly more dense base element packing. The later base was essentially similar to the earlier one, except the center to center spacing was $3.18 \mathrm{~mm}$, rather than $3.97 \mathrm{~mm}$. A comparison of the flows obtained for the two bases, with all other variables remaining the same, is shown in Figure 11. The net effect of the closer spacing of base beads is greater slip and flow velocity.

Temporal fluctuations in velocity were observed on a variety of time scales for most flows. Naturally in collisional flows there is a distribution of velocities amongst the different grains, as well as changes in each grain's velocity in time. There were also fluctuations occurring on longer time scales. These are believed to be related to periodic waves in the chute. As an example, we will present the temporal variations in velocity as measured by the fiber optic probe, for the fully developed collisional flow achieved at an angle of 23 degrees. Recall that the probe measures velocity in a spatial voxel on the order of one grain volume, so the measured velocity variations correspond closely to the velocities of individual grains. A 68 second long time series of velocity is shown in Figure 12a. Fluctuations are evident on a variety of time scales. The FFT of this time series shows the frequency content of the temporal variations, as shown in Figure 13a. There is a peak near $\mathrm{Hz}$, which presumable corresponds to a resonant periodic wave. At this time the value of the peak frequency is not fully understood. A second example is presented for the fully developed frictional flow achieved at an inclination of 21.4 degrees. Figure $12 \mathrm{~b}$ and Figure $13 \mathrm{~b}$ show the time series of velocity and its FFT. For this flow, the peak is not as pronounced, and the spectrum is wider. The waves causing these fluctuations do not seem to have any preferred period or resonance, though they certainly still exist.

\section{Conclusions}

An inclined chute facility and its associated diagnostics has been developed and utilized to study the flow of granular materials. A variety of flow regimes and flow phenomena were observed. Fully developed flows were observed over a bumpy base for a range of slopes. Under some conditions, these flows were dominated by friction and under other conditions, collisions played a dominant role. A variety of unsteady flows were also observed. These include decelerating flows, accelerating flows, and wavy (periodic) flows. The characteristics of the base strongly influenced the flow regime and flow dynamics. 


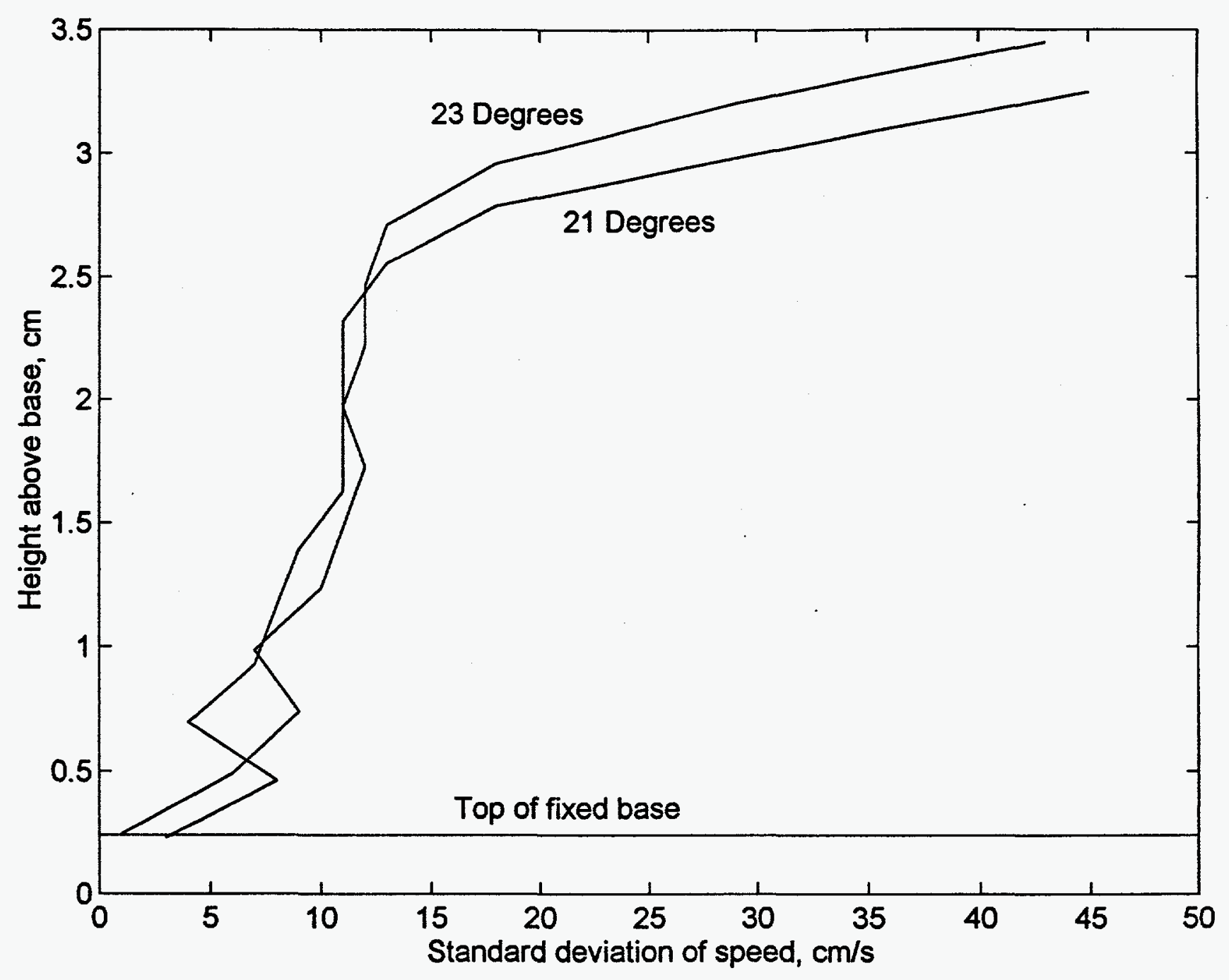

Fig. 10: Profile of velocity standard deviations at side wall for $21^{\circ}$ and $23^{\circ}$. 


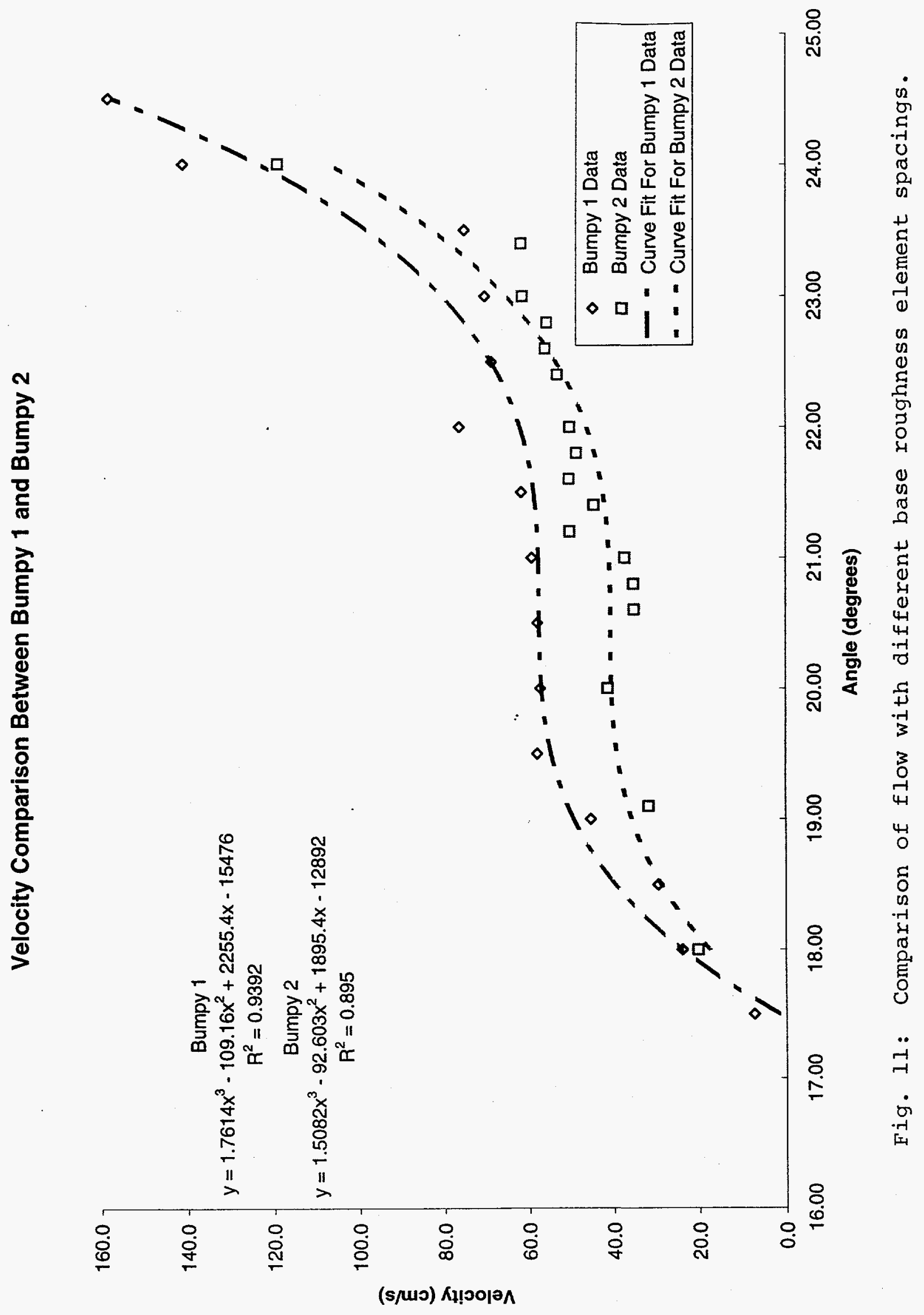




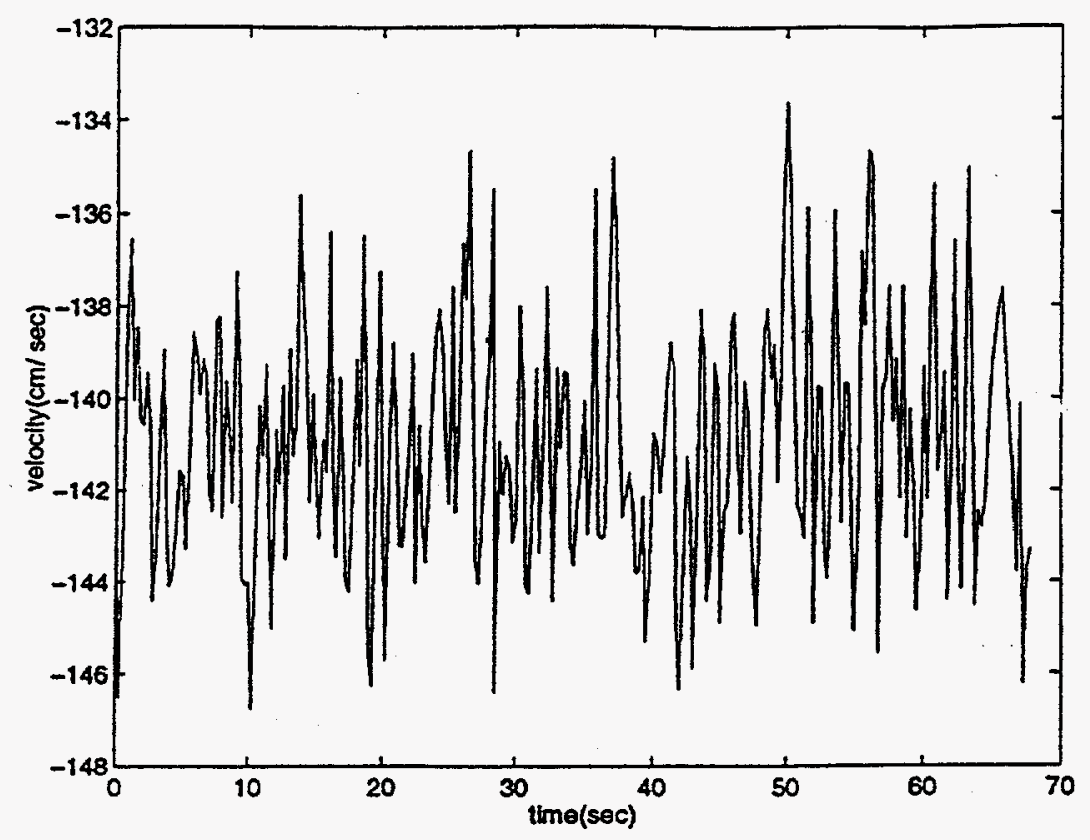

Fig. 12a: Time series of particle velocity for collisional flow.

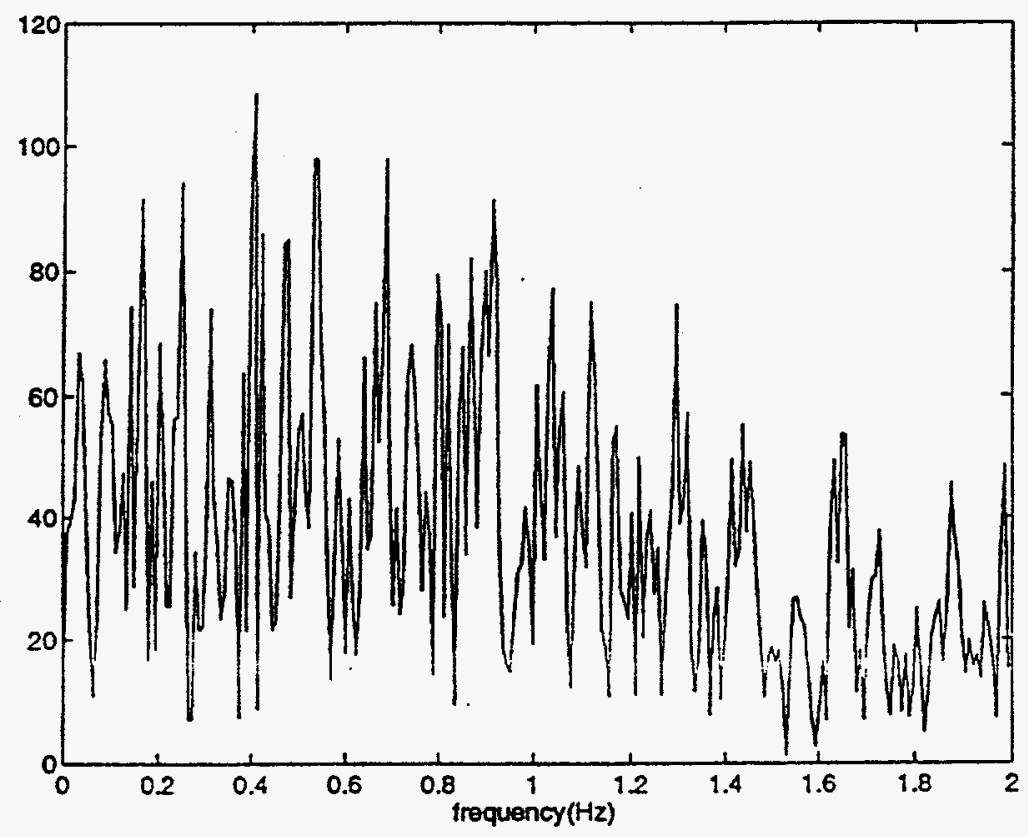

Fig. 12b: FFT of velocity. 


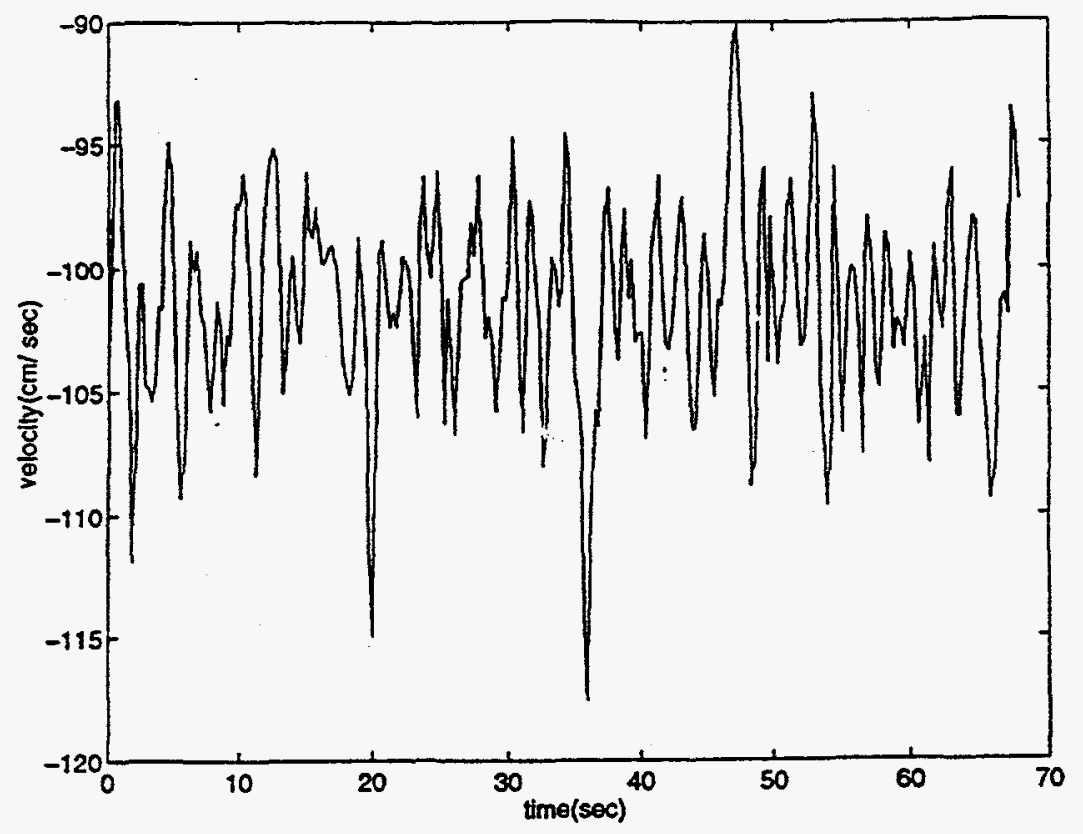

Fig. 13a: Time series of particle velocity for frictional flow.

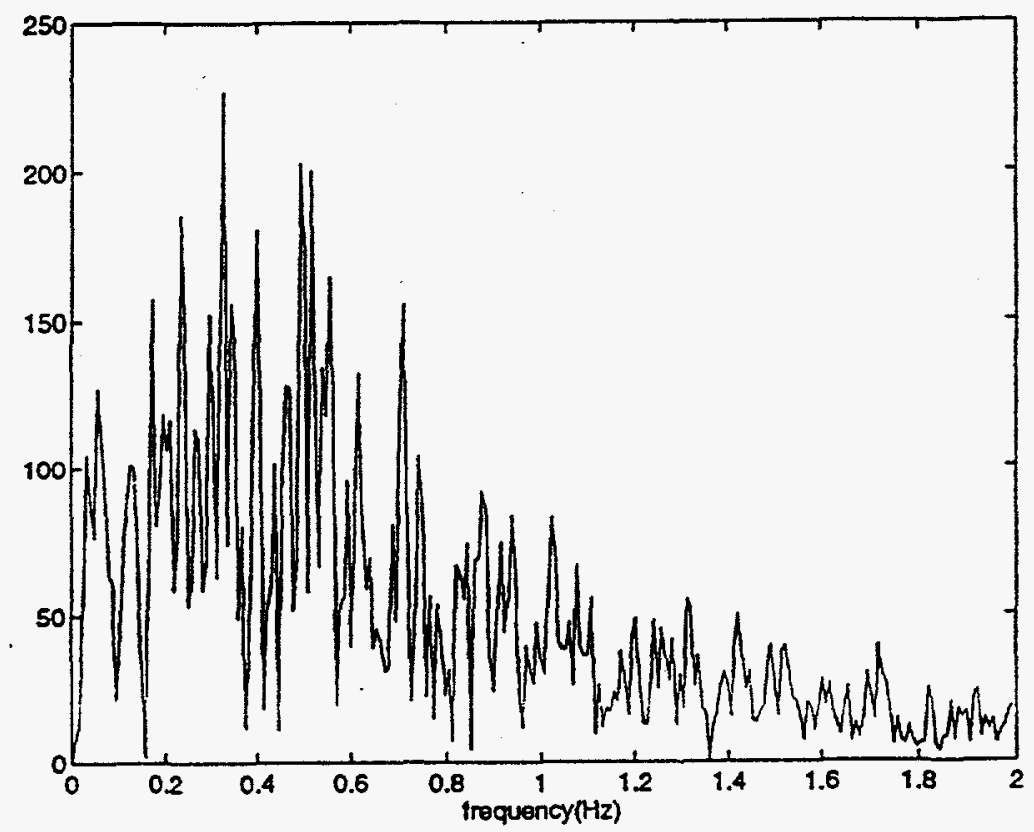

Fig. 13b: FFT of velocity. 


\section{References}

Ahn, H., C.E. Brennen, and R.H. Sabersky, "Measurements of the velocity, velocity fluctuation, density, and stresses for chute flows of granular material", Journal of Applied Mechanics, ASME, v. 58, 1991, 792.

Augenstein, D.A. and R. Hogg, "An experimental study of the flow of dry powders over inclined surfaces", Powder Technology, v. 19, 1978, 205-215.

Drake, T.G., "Granular flow: physical experiments and their implications for microstructural theories", Journal of Fluid Mechanics, v. 225, 1991, 121.

Hanes, D.M., J.T. Jenkins, and M.W. Richman, "The thickness of steady planes shear flows of circular disks between identical boundaries", Journal of Applied Mechanics, ASME, v. 55, No. 4, 1988, 969-974.

Hungr, O. and N.R. Morgenstern, "Experiments on the flow behaviour of granular materials at high velocity in an open channel", Geotechnique, v. 34, No. 3, $1984,405-413$.

Ishida, M. and T. Shirai, "Velocity distributions in the flow of solid particles in an inclined open channel", Journal of Chemical Engineering of Japan, v. 12, 1978, 46-50.

Johnson, P.C., P. Nott, and R. Jackson, "Frictional-collisional equations of motion for particulate flows and their application to chutes", Journal of Fluid Mechanics, v. $210,1990,501-535$.

Patton, J.S., C.E. Brennen, and R.H. Sabersky, "Shear flows of rapidly flowing granular materials", Journal of Applied Mechanics, American Society of Mechanical Engineers, Paper No. 87-WA/APM-27, 1987.

Ridgeway, K. and R. Rupp, "Flow of granular material down chutes", Chemical and Process Engineering, 1970, 82-85.

Roberts, A.W., "An investigation of the gravity flow of noncohesive granular materials through discharge chutes”, Journal of Engineering for Industry, ASME, 1969, 373-381.

Savage, S.B., "Gravity flow of cohesionless granular materials in chutes and channels", Journal of Fluid Mechanics, v. 92, 1979, 53-96.

Suzuki, A. and T. Tanaka, "Measurement of flow properties of powders along an inclined plane”, Ind. Eng. Chem. Fund., v. 10, 1971, 84-91. 
Appendix 1: Data Summary 
EXP: $\quad$ Experiment number identification

BASE: $\quad$ B1:bumpy1, B2:bumpy2; sm: smooth aluminum

GATE: $\quad$ Entrance sluice gate height, inches

MTOT: $\quad$ Total weight of flow material in system, pounds

ANGLE: $\quad$ Angle of chute inclination, degrees

TEMP: $\quad$ Room Temperature, ${ }^{\circ} \mathrm{F}$

HUMID: $\quad$ Room humidity, \%

CAM: $\quad$ High speed camera: $n$ (no) or $y$ (yes)

FIBER: $\quad$ Fiber optic sensors utilized: $n(n o)$ or $y(y e s)$

QUAL: Overall quality control index. 1:good; $0, \mathrm{~h}, \mathrm{t}:$ bad

STATE: Classification of flow regime: NF-no flow; US-unsteady flow, SC-steady collisional, SF-steady frictional, EA-expanding/accelerating

AH2-AH7: $\quad$ Average flow thickness in sections 2 through 7, cm

S1W-S7W: Weight of flow material in sections 2 through $7, \mathrm{~kg}$

AVF2-AVF7: Average volume fraction in sections 2 through 7

S2V-S7V: $\quad$ Average velocity in sections 7 through 7, nondimensional

S2MH-S7MH: Mass hold-up in section 2 through 7, nondimensional

MDOT: Discharge rate, nondimensional 
Data Summary

\begin{tabular}{|c|c|c|c|c|c|c|c|c|c|c|c|}
\hline DATE & EXP & BASE & GATE & MTOT & ANGLE & TEMP & HUMID & CAM & FIBER & QUAL & STATE \\
\hline $1 / 19 / 93$ & $00 \mathrm{~b}$ & $\mathrm{sm}$ & 1.00 & 250 & 14.20 & 77.9 & 43.0 & $n$ & \begin{tabular}{|l|}
$\mathbf{n}$ \\
\end{tabular} & 1 & \\
\hline $1 / 19 / 93$ & $00 c$ & sm & 1.50 & 250 & 14.20 & 77.9 & 50.0 & $n$ & $n$ & 1 & \\
\hline $1 / 19 / 93$ & $00 \mathrm{~d}$ & sm & 2.00 & 250 & 14.20 & 77.9 & 44.0 & $n$ & $n$ & 1 & \\
\hline $1 / 19 / 93$ & $00 \mathrm{e}$ & sm & 9.00 & 250 & 14.20 & 77.9 & 50.0 & $\mathrm{n}$ & $n$ & 0 & \\
\hline $1 / 19 / 93$ & oof & sm & 9.00 & 250 & & 0.0 & 45.0 & & & 1 & \\
\hline $1 / 21 / 93$ & $00 \mathrm{~g}$ & sm & 0.50 & 250 & 14.20 & 77.9 & 64.0 & $n$ & $n$ & 1 & \\
\hline $1 / 21 / 93$ & OOh & $\mathrm{sm}$ & 0.50 & 250 & 14.20 & 77.9 & 50.0 & $n$ & $n$ & 1 & \\
\hline $1 / 25 / 93$ & DOi & $\mathrm{sm}$ & 9.00 & 250 & 14.20 & 77.9 & 50.0 & $n$ & $n$ & 0 & \\
\hline $1 / 27 / 93$ & $00 j$ & $\mathrm{sm}$ & 2.00 & 250 & 14.20 & 77.9 & 50.0 & $n$ & $n$ & 1 & \\
\hline $1 / 29 / 93$ & DOK & $\mathrm{sm}$ & 1.50 & 250 & 14.20 & 77.9 & 50.0 & $n$ & $n$ & 1 & \\
\hline $1 / 31 / 93$ & 001 & sm & 0.50 & 250 & 14.20 & 77.9 & 50.0 & $y$ & $n$ & 1 & \\
\hline $1 / 31 / 93$ & $00 \mathrm{~m}$ & $\mathrm{sm}$ & 1.00 & 250 & 14.20 & 77.9 & 50.0 & $y$ & $n$ & 1 & \\
\hline $2 / 10 / 93$ & $00 n$ & sm & 0.50 & 250 & 11.70 & 77.9 & 65.0 & $n$ & $n$ & 1 & \\
\hline $2 / 10 / 93$ & 000 & sm & 0.50 & 250 & 11.70 & 77.9 & 65.0 & $n$ & $n$ & 1 & \\
\hline $2 / 11 / 93$ & $00 p$ & sm & 1.00 & 250 & 11.70 & 77.9 & 65.0 & $n$ & $n$ & 1 & \\
\hline $2 / 11 / 93$ & $00 \mathrm{q}$ & $\mathrm{sm}$ & 1.00 & 250 & 11.70 & 77.9 & 65.0 & $n$ & $n$ & 1 & \\
\hline $2 / 11 / 93$ & $00 r$ & $\mathrm{sm}$ & 1.50 & 250 & 11.70 & 77.9 & 65.0 & $n$ & $n$ & 1 & \\
\hline $2 / 12 / 93$ & $00 \mathrm{~s}$ & $\mathrm{sm}$ & 2.00 & 250 & 11.70 & 77.9 & 66.0 & $n$ & $n$ & 1 & \\
\hline $2 / 12 / 93$ & $00 t$ & sm & 9.00 & 250 & 11.70 & 77.9 & 65.0 & $n$ & $n$ & 0 & \\
\hline $2 / 12 / 93$ & $00 u$ & $\mathrm{sm}$ & 9.00 & 250 & 11.70 & 77.9 & 65.0 & $n$ & $n$ & 0 & \\
\hline $2 / 23 / 93$ & $00 v$ & b2 & 9.00 & 330 & 22.20 & 77.9 & 35.0 & $n$ & $n$ & $\bar{h}$ & \\
\hline $2 / 24 / 93$ & $00 w$ & b2 & 0.50 & 330 & 22.20 & 77.9 & 20.0 & $n$ & $n$ & 0 & NF \\
\hline $2 / 24 / 93$ & $00 x$ & b2 & 1.00 & 330 & 22.20 & 77.9 & 20.0 & $n$ & $n$ & $\bar{h}$ & US \\
\hline $2 / 24 / 93$ & $00 y$ & b2 & 1.50 & 330 & 22.20 & 77.9 & 20.0 & $n$ & $n$ & $\bar{h}$ & SC \\
\hline $2 / 24 / 93$ & $00 z$ & b2 & 2.00 & 330 & 22.20 & 77.9 & 20.0 & $n$ & $n$ & h & US \\
\hline $3 / 2 / 93$ & $01 a$ & b2 & 0.50 & 330 & 22.20 & 77.9 & 63.0 & $n$ & $n$ & 1 & NF \\
\hline $3 / 2 / 93$ & $01 b$ & b2 & 1.00 & 330 & 22.20 & 77.9 & 63.0 & $y$ & $n$ & 1 & US \\
\hline $3 / 2 / 93$ & $01 c$ & b2 & 1.50 & 330 & 22.20 & 77.9 & 63.0 & $n$ & $n$ & 0 & SC \\
\hline $3 / 2 / 93$ & 01d & b2 & 2.00 & 330 & 22.20 & 77.9 & 63.0 & $n$ & n & 1 & US \\
\hline $3 / 2 / 93$ & $01 e$ & b2 & 9.00 & 330 & 22.20 & 77.9 & 64.0 & $n$ & $n$ & 0 & \\
\hline $3 / 2 / 93$ & $01 \mathrm{~g}$ & b2 & 1.00 & 330 & 22.20 & 77.9 & 66.0 & $\bar{n}$ & $n$ & & US \\
\hline $3 / 3 / 93$ & $01 \mathrm{~h}$ & b2 & 1.50 & 330 & 22.20 & 77.9 & 66.0 & $n$ & $n$ & & SC \\
\hline $3 / 3 / 93$ & $01 f$ & b2 & 0.50 & 330 & 22.20 & 77.9 & 66.0 & $n$ & $n$ & & NF \\
\hline $3 / 4 / 93$ & $01 k$ & b2 & 2.00 & 330 & 20.00 & 77.9 & 65.0 & $n$ & $n$ & 0 & SF \\
\hline $3 / 9 / 93$ & $01 \mathrm{i}$ & b2 & 1.00 & 330 & 20.00 & 77.9 & 65.0 & $n$ & $n$ & 1 & SF \\
\hline $3 / 9 / 93$ & $01 j$ & b2 & 1.50 & 330 & 20.00 & 77.9 & 65.0 & $n$ & $n$ & 0 & SF \\
\hline $3 / 9 / 93$ & 011 & b2 & 9.00 & 330 & 20.00 & 77.9 & 64.0 & $n$ & $n$ & 0 & \\
\hline $3 / 9 / 93$ & $01 \mathrm{~m}$ & b2 & 0.50 & 330 & 20.00 & 77.9 & 63.0 & $n$ & $n$ & & NF \\
\hline $3 / 9 / 93$ & $01 n$ & b2 & 1.00 & 330 & 20.00 & 77.9 & 63.0 & $n$ & $n$ & & SF \\
\hline $3 / 9 / 93$ & 010 & b2 & 9.00 & 330 & 20.00 & 77.9 & 63.0 & $n$ & $n$ & & \\
\hline $3 / 15 / 93$ & $01 p$ & b2 & 0.50 & 330 & 24.00 & 77.9 & 60.0 & $n$ & $n$ & 1 & $E A$ \\
\hline $3 / 15 / 93$ & 019 & b2 & 1.00 & 330 & 24.00 & 77.9 & 59.0 & $n$ & $n$ & 1 & $E A$ \\
\hline $3 / 15 / 93$ & $01 r$ & b2 & 1.50 & 330 & 24.00 & 77.9 & 50.0 & $n$ & $n$ & 1 & EA \\
\hline $3 / 15 / 93$ & $01 \mathrm{~s}$ & b2 & 2.00 & 330 & 24.00 & 77.9 & 50.0 & $n$ & $n$ & 1 & $\overline{E A}$ \\
\hline $3 / 15 / 93$ & 011 & b2 & 9.00 & 330 & 24.00 & 77.9 & 61.0 & $n$ & $n$ & 0 & \\
\hline $3 / 15 / 93$ & 014 & b2 & 0.50 & 330 & 24.00 & 77.9 & & $n$ & $n$ & & $E A$ \\
\hline $3 / 15 / 93$ & $01 v$ & b2 & 1.00 & 330 & 24.00 & 77.9 & 63.0 & $n$ & $n$ & & EA \\
\hline $3 / 15 / 93$ & $01 w$ & b2 & 1.50 & 330 & 24.00 & 77.9 & 62.0 & $n$ & $n$ & & EA \\
\hline $3 / 18 / 93$ & $01 x$ & b2 & 0.50 & 330 & 18.00 & 77.9 & 66.0 & $n$ & $n$ & & \\
\hline $3 / 18 / 93$ & 01y & b2 & 1.00 & 330 & 18.00 & 77.9 & 62.0 & $n$ & $\mathrm{n}$ & 0 & \\
\hline
\end{tabular}




\begin{tabular}{|c|c|c|c|c|c|c|c|c|c|c|c|}
\hline DATE & EXP & BASE & GATE & MTOT & ANGLE & TEMP & HUMID & CAM & FIBER & QUAL & $\overline{\text { STATE }}$ \\
\hline $3 / 18 / 93$ & 012 & b2 & 1.00 & 330 & 18.00 & 77.9 & 66.0 & $n$ & $n$ & & \\
\hline $3 / 18 / 93$ & $02 a$ & b2 & 1.50 & 330 & 18.00 & 77.9 & 60.0 & $n$ & $n$ & 1 & \\
\hline $3 / 18 / 93$ & $02 b$ & b2 & 2.00 & 330 & 18.00 & 77.9 & 63.0 & $n$ & $n$ & 1 & \\
\hline $3 / 18 / 93$ & $02 c$ & b2 & 9.00 & 330 & 18.00 & 77.9 & 63.0 & $n$ & $n$ & 0 & \\
\hline $3 / 18 / 93$ & 02d & b2 & 9.00 & 330 & 18.00 & 77.9 & 68.0 & $n$ & $n$ & & \\
\hline $3 / 19 / 93$ & $02 e$ & b2 & 9.00 & 330 & 16.20 & 77.9 & 59.0 & $n$ & $n$ & 0 & \\
\hline $3 / 22 / 93$ & $02 f$ & b2 & 0.50 & 330 & 28.20 & 77.9 & 64.0 & $n$ & $n$ & & EA \\
\hline $3 / 22 / 93$ & $02 g$ & $b 2$ & 1.00 & 330 & 28.20 & 77.9 & 65.0 & $n$ & $n$ & & $\overline{E A}$ \\
\hline $3 / 22 / 93$ & $02 \mathrm{~h}$ & b2 & 1.50 & 330 & 28.20 & 77.9 & 66.0 & $n$ & $\bar{n}$ & & $\overline{E A}$ \\
\hline $3 / 22 / 93$ & $02 i$ & 62 & 0.50 & 330 & 28.20 & 77.9 & 67.0 & $n$ & $n$ & 1 & $E A$ \\
\hline $3 / 22 / 93$ & $09 z$ & b2 & 2.00 & 330 & 23.40 & 77.9 & 66.0 & $y$ & $y$ & 1 & SC \\
\hline $3 / 23 / 93$ & $02 j$ & b2 & 1.00 & 330 & 28.20 & 77.9 & 70.0 & $n$ & $n$ & 1 & $E A$ \\
\hline $3 / 23 / 93$ & $02 k$ & b2 & 1.50 & 330 & 28.20 & 77.9 & 70.0 & $n$ & $n$ & 1 & $\mathrm{EA}$ \\
\hline $3 / 23 / 93$ & 021 & $\mathrm{b2}$ & 2.00 & 330 & 28.20 & 77.9 & 70.0 & $n$ & $n$ & & $E A$ \\
\hline $3 / 23 / 93$ & $02 m$ & b2 & 9.00 & 330 & 28.20 & 77.9 & 70.0 & $n$ & $n$ & 0 & EA \\
\hline $3 / 24 / 93$ & $02 n$ & b2 & 0.50 & 330 & 26.20 & 77.9 & 71.0 & $n$ & $n$ & $\bar{w}$ & EA \\
\hline $3 / 24 / 93$ & 020 & b2 & 1.00 & 330 & 26.20 & 77.9 & 71.0 & $n$ & $n$ & & EA \\
\hline $3 / 24 / 93$ & $02 p$ & b2 & 9.00 & 330 & 26.20 & 77.9 & 71.0 & $n$ & $n$ & & EA \\
\hline $3 / 24 / 93$ & $02 q$ & b2 & 0.50 & 330 & 26.20 & 77.9 & 71.0 & $\bar{n}$ & $n$ & 1 & $\overline{E A}$ \\
\hline $3 / 24 / 93$ & $02 r$ & b2 & 1.00 & 330 & 26.20 & 77.9 & 71.0 & $n$ & $n$ & 1 & EA \\
\hline $3 / 24 / 93$ & $02 \mathrm{~s}$ & b2 & 1.50 & 330 & 26.20 & 77.9 & 71.0 & $n$ & $n$ & 1 & EA \\
\hline $3 / 24 / 93$ & $02 t$ & b2 & 2.00 & 330 & 26.20 & 77.9 & 71.0 & $n$ & $n$ & 1 & EA \\
\hline $3 / 24 / 93$ & $02 u$ & b2 & 9.00 & 330 & 26.20 & 77.9 & 71.0 & $n$ & $n$ & 0 & EA \\
\hline $3 / 24 / 93$ & $02 v$ & b2 & 0.50 & 330 & 27.20 & 77.9 & 68.0 & $n$ & $n$ & 1 & EA \\
\hline $3 / 24 / 93$ & $02 w$ & b2 & 1.00 & 330 & 27.20 & 77.9 & 68.0 & $n$ & $n$ & 1 & EA \\
\hline $3 / 25 / 93$ & $02 x$ & b2 & 1.50 & 330 & 27.20 & 77.9 & 68.0 & $\mathbf{n}$ & $n$ & 1 & EA \\
\hline $3 / 25 / 93$ & $02 y$ & $\mathrm{~b} 2$ & 2.00 & 330 & 27.20 & 77.9 & 68.0 & $\bar{n}$ & $n$ & 1 & $\overline{E A}$ \\
\hline $3 / 25 / 93$ & 022 & b2 & 9.00 & 330 & 27.20 & 77.9 & 68.0 & $n$ & $n$ & 0 & $\overline{E A}$ \\
\hline $3 / 25 / 93$ & $03 a$ & $\mathrm{b2}$ & 0.50 & 330 & 27.20 & 77.9 & 68.0 & $n$ & $\bar{n}$ & & EA \\
\hline $3 / 25 / 93$ & $03 b$ & b2 & 1.00 & 330 & 27.20 & 77.9 & 68.0 & $n$ & $n$ & 1 & $\overline{E A}$ \\
\hline $3 / 25 / 93$ & $03 c$ & b2 & 1.50 & 330 & 27.20 & 77.9 & 68.0 & $\bar{n}$ & $\mathbf{n}$ & 1 & EA \\
\hline $3 / 25 / 93$ & 03d & $\mathrm{b} 2$ & 0.50 & 330 & 25.30 & 77.9 & 67.0 & $n$ & $n$ & 1 & EA \\
\hline $3 / 25 / 93$ & $03 e$ & b2 & 1.00 & 330 & 25.30 & 77.9 & 67.0 & $n$ & $n$ & 1 & EA \\
\hline $3 / 25 / 93$ & $03 f$ & $\mathrm{b2}$ & 1.50 & 330 & 25.30 & 77.9 & 67.0 & $n$ & $\mathbf{n}$ & 1 & $\overline{E A}$ \\
\hline $3 / 25 / 93$ & $\overline{03 g}$ & b2 & 2.00 & 330 & 25.30 & 77.9 & 67.0 & $n$ & $n$ & 1 & EA \\
\hline $3 / 25 / 93$ & $03 \mathrm{~h}$ & b2 & 9.00 & 330 & 25.30 & 77.9 & 67.0 & $n$ & $n$ & 0 & $E A$ \\
\hline $3 / 26 / 93$ & $\overline{03 i}$ & b2 & 0.50 & 330 & 25.30 & 77.9 & 64.0 & $n$ & $n$ & $\overline{1}$ & EA \\
\hline $3 / 26 / 93$ & $03 j$ & b2 & 1.00 & 330 & 25.30 & 77.9 & 64.0 & $n$ & $n$ & 1 & EA \\
\hline $3 / 26 / 93$ & $03 k$ & b2 & 1.50 & 330 & 25.30 & 77.9 & 64.0 & $n$ & $n$ & 1 & $\overline{E A}$ \\
\hline $3 / 26 / 93$ & 031 & b2 & 0.50 & 330 & 23.10 & 77.9 & 65.0 & $n$ & $n$ & 1 & \\
\hline $3 / 26 / 93$ & $03 m$ & $\mathrm{b2}$ & 1.00 & 330 & 23.10 & 77.9 & 65.0 & $\bar{n}$ & $n$ & 1 & \\
\hline $3 / 26 / 93$ & $03 n$ & b2 & 1.50 & 330 & 23.10 & 77.9 & 65.0 & $n$ & $n$ & 1 & \\
\hline $3 / 26 / 93$ & 030 & b2 & 2.00 & 330 & 23.10 & 77.9 & 65.0 & $n$ & $n$ & 1 & \\
\hline $3 / 26 / 93$ & $03 p$ & b2 & 9.00 & 330 & 23.10 & 77.9 & 65.0 & $n$ & $n$ & 0 & \\
\hline $3 / 26 / 93$ & $03 q$ & b2 & 0.50 & 330 & 23.10 & 77.9 & 65.0 & $n$ & $\bar{n}$ & & \\
\hline $3 / 26 / 93$ & $03 r$ & b2 & 1.00 & 330 & 23.10 & 77.9 & 65.0 & $\bar{n}$ & $\bar{n}$ & & \\
\hline $3 / 26 / 93$ & $03 \mathrm{~s}$ & $\mathrm{b2}$ & 9.00 & 330 & 23.10 & 77.9 & 65.0 & $n$ & $n$ & & \\
\hline $3 / 29 / 93$ & $03 t$ & b2 & 0.50 & 330 & 21.20 & 77.9 & 65.0 & $n$ & $n$ & & NF \\
\hline $3 / 29 / 93$ & $03 u$ & b2 & 1.00 & 330 & 21.20 & 77.9 & 65.0 & $n$ & $n$ & & SF \\
\hline $3 / 29 / 93$ & $03 v$ & $\mathrm{b2}$ & 9.00 & 330 & 21.20 & 77.9 & 65.0 & $n$ & $n$ & & \\
\hline
\end{tabular}




\begin{tabular}{|c|c|c|c|c|c|c|c|c|c|c|c|}
\hline DATE & EXP & BASE & GATE & MTOT & ANGLE & TEMP & HUMID & CAM & FIBER & QUAL & STATE \\
\hline $3 / 29 / 93$ & $03 w$ & b2 & 0.50 & 330 & 21.20 & 77.9 & 66.0 & $n$ & $\mathbf{n}$ & 0 & NF \\
\hline $3 / 29 / 93$ & $03 x$ & b2 & 1.00 & 330 & 21.20 & 77.9 & 66.0 & $n$ & $n$ & 1 & SF \\
\hline $3 / 29 / 93$ & $03 y$ & b2 & 1.50 & 330 & 21.20 & 77.9 & 66.0 & $n$ & $n$ & 1 & \\
\hline $3 / 29 / 93$ & $03 z$ & $\mathrm{~b} 2$ & 2.00 & 330 & 21.20 & 77.9 & 66.0 & $n$ & $n$ & 1 & SF \\
\hline $3 / 29 / 93$ & $04 a$ & b2 & 9.00 & 330 & 21.20 & 77.9 & 65.0 & $n$ & $n$ & 0 & \\
\hline $3 / 30 / 93$ & $04 b$ & b2 & 1.00 & 330 & 19.10 & 77.9 & 62.0 & $n$ & $n$ & 1 & \\
\hline $3 / 30 / 93$ & $04 c$ & b2 & 1.50 & 330 & 19.10 & 77.9 & 62.0 & $n$ & $n$ & 1 & \\
\hline $3 / 30 / 93$ & $04 d$ & b2 & 2.00 & 330 & 19.10 & 77.9 & 62.0 & $n$ & $n$ & 1 & \\
\hline $3 / 30 / 93$ & $04 e$ & b2 & 9.00 & 330 & 19.10 & 77.9 & 62.0 & $n$ & $n$ & 0 & \\
\hline $3 / 30 / 93$ & $04 f$ & b2 & 0.50 & 3.30 & 19.10 & 77.9 & 61.0 & $\bar{n}$ & $n$ & 1 & \\
\hline $3 / 30 / 93$ & $04 \mathrm{~g}$ & b2 & 1.00 & 330 & 19.10 & 77.9 & 61.0 & $n$ & $n$ & 1 & \\
\hline $3 / 30 / 93$ & $04 h$ & b2 & 9.00 & 330 & 19.10 & 77.9 & 62.0 & $n$ & $n$ & 0 & \\
\hline $3 / 30 / 93$ & $04 i$ & b2 & 9.00 & 330 & 17.10 & 77.9 & 62.0 & $n$ & $n$ & 0 & \\
\hline $3 / 30 / 93$ & $04 j$ & b2 & 9.00 & 330 & 17.10 & 77.9 & 62.0 & $n$ & $n$ & 0 & \\
\hline $4 / 1 / 93$ & $04 n$ & b2 & 2.00 & 330 & 20.00 & 77.9 & 66.0 & & & 1 & SF \\
\hline $4 / 1 / 93$ & $04 k$ & b2 & 2.00 & 330 & 22.20 & 77.9 & 65.0 & $n$ & $n$ & 1 & US \\
\hline $4 / 1 / 93$ & 041 & b2 & 9.00 & 330 & 22.20 & 77.9 & 64.0 & $n$ & $n$ & 0 & \\
\hline $4 / 1 / 93$ & $04 \mathrm{~m}$ & b2 & 9.00 & 330 & 22.20 & 77.9 & 65.0 & $n$ & $n$ & & \\
\hline $4 / 1 / 93$ & 040 & b2 & 9.00 & 330 & 20.00 & 77.9 & 65.0 & $n$ & $n$ & 0 & \\
\hline $4 / 1 / 93$ & $04 p$ & b2 & 9.00 & 330 & 20.00 & 77.9 & 66.0 & $n$ & $n$ & 0 & \\
\hline $4 / 1 / 93$ & $04 q$ & b2 & 1.00 & 330 & 17.10 & 77.9 & 67.0 & $n$ & $n$ & 0 & \\
\hline $4 / 2 / 93$ & $04 r$ & b2 & 9.00 & 330 & 28.20 & 77.9 & 52.0 & $n$ & $n$ & 0 & EA \\
\hline $4 / 5 / 93$ & $04 \mathrm{~s}$ & b2 & 1.50 & 330 & 28.20 & 77.9 & 62.0 & $\mathbf{n}$ & $n$ & 1 & EA \\
\hline $4 / 5 / 93$ & $04 t$ & b2 & 1.00 & 330 & 28.20 & 77.9 & 62.0 & $n$ & $n$ & 1 & $E A$ \\
\hline $4 / 5 / 93$ & $04 u$ & b2 & 1.50 & 330 & 28.20 & 77.9 & 62.0 & $n$ & $n$ & 1 & $E A$ \\
\hline $4 / 5 / 93$ & $04 v$ & b2 & 0.50 & 330 & 22.20 & 77.9 & 67.0 & $n$ & $n$ & & NF \\
\hline $4 / 5 / 93$ & $04 w$ & b2 & 1.00 & 330 & 22.20 & 77.9 & 67.0 & $n$ & $n$ & & US \\
\hline $4 / 5 / 93$ & $04 x$ & b2 & 9.00 & 330 & 22.20 & 77.9 & 67.0 & & & 0 & \\
\hline $4 / 5 / 93$ & $04 y$ & b2 & 1.00 & 330 & 20.00 & 77.9 & 65.0 & $n$ & $n$ & 1 & SF \\
\hline $4 / 5 / 93$ & 042 & $b^{2} 2$ & 1.50 & 330 & 20.00 & 77.9 & 65.0 & $n$ & $n$ & 1 & SF \\
\hline $4 / 5 / 93$ & $05 a$ & b2 & 1.00 & 330 & 20.00 & 77.9 & 65.0 & $n$ & $n$ & & SF \\
\hline $4 / 5 / 93$ & $05 b$ & b2 & 2.00 & 330 & 20.00 & 77.9 & 65.0 & $n$ & $n$ & 1 & SF \\
\hline $4 / 7 / 93$ & 05c & b2 & 9.00 & 330 & 24.00 & 77.9 & 58.0 & $n$ & $n$ & 0 & \\
\hline $4 / 7 / 93$ & $05 d$ & b2 & 0.50 & 330 & 24.00 & 77.9 & 58.0 & $n$ & $n$ & & $\overline{E A}$ \\
\hline $4 / 7 / 93$ & $05 \mathrm{e}$ & b2 & 1.00 & 330 & 24.00 & 77.9 & 58.0 & $n$ & $n$ & & $\overline{E A}$ \\
\hline $4 / 7 / 93$ & $05 f$ & b2 & 9.00 & 330 & 24.00 & 77.9 & 58.0 & $n$ & $n$ & & \\
\hline $4 / 12 / 93$ & 050 & b2 & 2.00 & 330 & 20.00 & 77.9 & 54.0 & $n$ & $n$ & 1 & SF \\
\hline $4 / 12 / 93$ & $05 h$ & b2 & 9.00 & 330 & 20.00 & 77.9 & 55.0 & $n$ & $n$ & 0 & \\
\hline $6 / 14 / 93$ & $05 n$ & b2 & 9.00 & 330 & 20.00 & 77.9 & 72.0 & $\mathrm{n}$ & $n$ & 0 & \\
\hline $6 / 14 / 93$ & 050 & b2 & 2.00 & 330 & 20.00 & 77.9 & 73.0 & $n$ & $n$ & $v$ & SF \\
\hline $6 / 14 / 93$ & $05 p$ & b2 & 2.00 & 330 & 20.00 & 77.9 & 73.0 & $n$ & $n$ & $v$ & SF \\
\hline $6 / 14 / 93$ & $05 q$ & b2 & 2.00 & 330 & 20.00 & 77.9 & 72.0 & $n$ & $n$ & $v$ & SF \\
\hline $6 / 14 / 93$ & $05 r$ & b2 & 2.00 & 330 & 20.00 & 77.9 & 72.0 & $n$ & $n$ & 1 & SF \\
\hline $6 / 14 / 93$ & $05 s$ & b2 & 1.50 & 330 & 20.00 & 77.9 & 71.0 & $n$ & $n$ & 1 & SF \\
\hline $6 / 15 / 93$ & $05 t$ & b2 & 9.00 & 330 & 20.00 & 77.9 & 71.0 & $n$ & $n$ & 0 & \\
\hline $6 / 15 / 93$ & $05 u$ & b2 & 2.00 & 330 & 20.00 & 77.9 & 69.0 & $n$ & $n$ & 1 & SF \\
\hline $6 / 16 / 93$ & $05 v$ & $\mathrm{~b} 2$ & 3.00 & 330 & 20.00 & 77.9 & 71.0 & $n$ & $n$ & 1 & \\
\hline $6 / 17 / 93$ & $05 w$ & $\mathrm{~b} 2$ & 2.00 & 330 & 20.00 & 77.9 & 68.0 & $n$ & $n$ & 1 & SF \\
\hline $6 / 17 / 93$ & $05 x$ & $\mathrm{b2}$ & 1.50 & 330 & 20.00 & 77.9 & 68.0 & $n$ & $n$ & 1 & SF \\
\hline $6 / 17 / 93$ & $05 y$ & b2 & 9.00 & 330 & 20.00 & 77.9 & 65.0 & $n$ & $n$ & 0 & \\
\hline
\end{tabular}


Data Summary

\begin{tabular}{|c|c|c|c|c|c|c|c|c|c|c|c|}
\hline DATE & EXP & BASE & GATE & MTOT & ANGLE & TEMP & HUMID & CAM & FIBER & QUAL & STATE \\
\hline $6 / 17 / 93$ & $05 z$ & b2 & 9.00 & 330 & 20.00 & 77.9 & 65.0 & $n$ & $n$ & 0 & \\
\hline $6 / 28 / 93$ & $06 a$ & b2 & 9.00 & 330 & 24.10 & 77.9 & 78.0 & $y$ & $n$ & 0 & \\
\hline $7 / 1 / 93$ & $06 b$ & b2 & 2.50 & 330 & 24.10 & 77.9 & 72.0 & $n$ & $n$ & 1 & \\
\hline $7 / 1 / 93$ & $06 c$ & b2 & 9.00 & 330 & 24.10 & 77.9 & 71.0 & $n$ & $n$ & 0 & \\
\hline $7 / 1 / 93$ & 06d & b2 & 2.50 & 330 & 23.80 & 77.9 & 71.0 & $n$ & $\mathbf{n}$ & 1 & \\
\hline $7 / 1 / 93$ & $06 \mathrm{e}$ & b2 & 9.00 & 330 & 23.80 & 77.9 & 71.0 & $n$ & $n$ & 0 & \\
\hline $7 / 6 / 93$ & $06 f$ & b2 & 1.37 & 330 & 23.40 & 77.9 & 69.0 & $\mathbf{n}$ & $n$ & 1 & EA \\
\hline $7 / 6 / 93$ & $06 \mathrm{~h}$ & $\mathrm{~b} 2$ & 2.00 & 330 & 23.00 & 77.9 & 69.0 & $n$ & $n$ & 1 & \\
\hline $7 / 8 / 93$ & $06 \mathrm{~g}$ & b2 & 1.25 & 330 & 23.20 & 77.9 & 69.0 & $n$ & $n$ & 1 & \\
\hline $7 / 8 / 93$ & $06 i$ & b2 & 1.50 & 330 & 22.80 & 77.9 & 72.0 & $n$ & $n$ & 1 & SC \\
\hline $7 / 8 / 93$ & $06 j$ & b2 & 9.00 & 330 & $22 . \overline{80}$ & 77.9 & 72.0 & $n$ & $\mathbf{n}$ & 0 & \\
\hline $7 / 8 / 93$ & $06 k$ & b2 & 2.50 & 330 & 22.60 & 77.9 & 71.0 & $n$ & $n$ & 1 & \\
\hline $7 / 8 / 93$ & 061 & b2 & 9.00 & 330 & 22.60 & 77.9 & 71.0 & $n$ & $n$ & 0 & \\
\hline $7 / 8 / 93$ & $06 \mathrm{~m}$ & b2 & 2.50 & 330 & 22.40 & 77.9 & 71.0 & $n$ & $n$ & 1 & \\
\hline $7 / 8 / 93$ & $06 n$ & $\mathrm{~b} 2$ & 9.00 & 330 & 22.40 & 77.9 & 71.0 & $n$ & $n$ & 0 & \\
\hline $7 / 8 / 93$ & 060 & $\mathrm{~b} 2$ & 2.25 & 330 & 22.20 & 77.9 & 71.0 & $n$ & $n$ & 1 & \\
\hline $7 / 12 / 93$ & $06 p$ & b2 & 9.00 & 330 & 22.20 & 77.9 & 70.0 & $\mathrm{n}$ & $n$ & 0 & \\
\hline $7 / 12 / 93$ & $06 q$ & $\mathrm{~b} 2$ & 2.00 & 330 & 22.00 & 77.9 & 70.0 & $n$ & $n$ & 1 & US \\
\hline $7 / 12 / 93$ & $06 r$ & b2 & 9.00 & 330 & 22.00 & 77.9 & 70.0 & $\mathrm{n}$ & $n$ & 0 & \\
\hline $7 / 12 / 93$ & $06 s$ & b2 & 1.87 & 330 & 21.80 & 77.9 & 70.0 & $n$ & $n$ & 1 & \\
\hline $7 / 12 / 93$ & $06 t$ & b2 & 9.00 & 330 & 21.80 & 77.9 & 70.0 & $n$ & $n$ & 0 & \\
\hline $7 / 12 / 93$ & $06 u$ & b2 & 1.87 & 330 & 21.60 & 77.9 & 70.0 & $n$ & $n$ & 1 & SF \\
\hline $7 / 12 / 93$ & $06 v$ & b2 & 9.00 & 330 & 21.60 & 77.9 & 68.0 & $n$ & $n$ & 0 & \\
\hline $7 / 13 / 93$ & $06 w$ & b2 & 2.25 & 330 & 21.40 & 77.9 & 72.0 & $n$ & $n$ & 1 & \\
\hline $7 / 13 / 93$ & $06 x$ & b2 & 9.00 & 330 & 21.40 & 77.9 & 71.0 & $n$ & $n$ & 0 & \\
\hline $7 / 13 / 93$ & $06 y$ & b2 & 2.12 & 330 & 21.20 & 77.9 & 71.0 & $n$ & $n$ & 1 & \\
\hline $7 / 13 / 93$ & $06 z$ & b2 & 9.00 & 330 & 21.20 & 77.9 & 71.0 & $n$ & $n$ & 0 & \\
\hline $7 / 17 / 93$ & $07 a$ & b2 & 2.00 & 330 & 21.00 & 77.9 & 76.0 & $n$ & $n$ & 1 & SF \\
\hline $7 / 17 / 93$ & $07 b$ & b2 & 9.00 & 330 & 21.00 & 77.9 & 76.0 & $n$ & $n$ & 0 & \\
\hline $7 / 17 / 93$ & 07c & b2 & 1.75 & 330 & 20.80 & 77.9 & 76.0 & $n$ & $n$ & 1 & SF \\
\hline $7 / 19 / 93$ & 07d & b2 & 9.00 & 330 & 20.80 & 77.9 & 69.0 & $n$ & $n$ & 0 & \\
\hline $7 / 19 / 93$ & $07 e$ & b2 & 1.00 & 330 & 20.80 & 77.9 & 68.0 & $y$ & $n$ & 0 & SF \\
\hline $7 / 19 / 93$ & $07 f$ & b2 & 9.00 & 330 & 20.80 & 77.9 & 69.0 & $y$ & $n$ & 0 & \\
\hline $7 / 19 / 93$ & $07 g$ & b2 & 2.00 & 330 & 20.60 & 77.9 & 69.0 & $n$ & $n$ & 1 & SF \\
\hline $7 / 19 / 93$ & $07 \mathrm{~h}$ & b2 & 9.00 & 330 & 20.60 & 77.9 & 70.0 & $n$ & $n$ & 0 & \\
\hline $7 / 20 / 93$ & $07 i$ & b2 & 2.00 & 330 & 20.40 & 77.9 & 71.0 & $n$ & $n$ & 1 & SF \\
\hline $7 / 20 / 93$ & $07 j$ & b2 & 9.00 & 330 & 20.40 & 77.9 & 71.0 & $n$ & $n$ & 0 & \\
\hline $7 / 20 / 93$ & $07 k$ & b2 & 1.75 & 330 & 20.20 & 77.9 & 71.0 & $n$ & $n$ & 1 & SF \\
\hline $7 / 20 / 93$ & $07 \mid$ & b2 & 9.00 & 330 & 20.20 & 77.9 & 72.0 & $n$ & $n$ & 0 & \\
\hline $7 / 20 / 93$ & $07 \mathrm{~m}$ & b2 & 1.75 & 330 & 20.00 & 77.9 & 72.0 & $n$ & $n$ & 1 & SF \\
\hline $7 / 20 / 93$ & $07 n$ & b2 & 9.00 & 330 & 20.00 & 77.9 & 71.0 & $n$ & $n$ & 0 & \\
\hline $7 / 28 / 93$ & 070 & b2 & 9.00 & 330 & 24.10 & 77.9 & 69.0 & $n$ & $n$ & 0 & \\
\hline $7 / 28 / 93$ & $07 p$ & b2 & 2.50 & 330 & 24.10 & 77.9 & 68.0 & $n$ & $n$ & 1 & \\
\hline $7 / 28 / 93$ & $07 q$ & b2 & 9.00 & 330 & 23.20 & 77.9 & 67.0 & $n$ & $n$ & 0 & \\
\hline $7 / 29 / 93$ & $07 r$ & b2 & 1.50 & 330 & 23.20 & 77.9 & 67.0 & $n$ & $n$ & 0 & \\
\hline $7 / 29 / 93$ & $07 s$ & b2 & 9.00 & 330 & 22.40 & 77.9 & 66.0 & $n$ & $n$ & 0 & \\
\hline $7 / 29 / 93$ & $07 t$ & b2 & 2.50 & 330 & 22.40 & 77.9 & 66.0 & $n$ & $n$ & 1 & \\
\hline $8 / 4 / 93$ & $07 u$ & b2 & 1.50 & 330 & 21.20 & 77.9 & 71.0 & $n$ & $n$ & 1 & \\
\hline $8 / 4 / 93$ & $07 v$ & b2 & 9.00 & 330 & 21.20 & 77.9 & 71.0 & $n$ & $n$ & 0 & \\
\hline $8 / 5 / 93$ & $07 w$ & $\mathrm{~b} 2$ & 2.50 & 330 & 24.10 & 77.9 & 68.0 & $n$ & $n$ & 1 & \\
\hline
\end{tabular}


Data Summary

\begin{tabular}{|c|c|c|c|c|c|c|c|c|c|c|c|}
\hline DATE & EXP & BASE & GATE & MTOT & ANGLE & TEMP & HUMID & CAM & FIBER & QUAL & STATE \\
\hline $8 / 5 / 93$ & $07 x$ & b2 & 9.00 & 330 & 24.10 & 77.9 & 69.0 & $n$ & $n$ & 0 & \\
\hline $8 / 5 / 93$ & $07 y$ & b2 & 2.50 & 330 & 24.10 & 77.9 & 69.0 & $n$ & $n$ & 1 & \\
\hline $8 / 12 / 93$ & $07 z$ & b2 & 9.00 & 330 & 24.10 & 77.9 & 68.0 & $n$ & $n$ & 0 & \\
\hline $8 / 12 / 93$ & $08 a$ & b2 & 1.50 & 330 & 21.20 & 77.9 & 67.0 & $n$ & $n$ & 0 & \\
\hline $8 / 12 / 93$ & $08 b$ & b2 & 9.00 & 330 & 21.20 & 77.9 & 67.0 & $n$ & $n$ & 0 & \\
\hline $8 / 17 / 93$ & $\overline{08 c}$ & b2 & 2.00 & 330 & 21.00 & 77.9 & 69.0 & $y$ & $n$ & 0 & SF \\
\hline $8 / 17 / 93$ & 08d & b2 & 2.00 & 330 & 21.00 & 77.9 & 69.0 & $y$ & $n$ & 0 & SF \\
\hline $8 / 17 / 93$ & $08 \mathrm{e}$ & $\mathrm{b} 2$ & 2.00 & 330 & 21.00 & 77.9 & 69.0 & $y$ & $n$ & 0 & SF \\
\hline $8 / 17 / 93$ & $08 f$ & b2 & 2.00 & 330 & 21.00 & 77.9 & 69.0 & $y$ & $n$ & 0 & SF \\
\hline $8 / 17 / 93$ & $08 \mathrm{n}$ & b2 & 2.00 & 330 & 21.00 & 77.9 & 69.0 & $y$ & $n$ & 0 & SF \\
\hline $8 / 17 / 93$ & $08 \mathrm{~h}$ & b2 & 2.00 & 330 & 21.00 & 77.9 & 69.0 & $y$ & $n$ & 0 & SF \\
\hline $8 / 17 / 93$ & $08 \mathbf{i}$ & b2 & 2.00 & 330 & 21.00 & 77.9 & 69.0 & $y$ & $n$ & 0 & SF \\
\hline $8 / 17 / 93$ & $08 j$ & b2 & 2.00 & 330 & 21.00 & 77.9 & 69.0 & $y$ & $n$ & 0 & SF \\
\hline $8 / 31 / 93$ & $08 k$ & b2 & 1.75 & 330 & 21.00 & 77.9 & 67.0 & $y$ & $n$ & 1 & SF \\
\hline 9/1/93 & 081 & b2 & 2.00 & 330 & 21.00 & 77.9 & 72.0 & $y$ & $n$ & 1 & SF \\
\hline $9 / 1 / 93$ & $08 \mathrm{~m}$ & b2 & 1.75 & 330 & 21.20 & 77.9 & 67.0 & $y$ & $n$ & 1 & SF \\
\hline 9/1/93 & $08 n$ & b2 & 2.00 & 330 & 21.20 & 77.9 & 67.0 & $y$ & $n$ & 1 & SF \\
\hline $9 / 2 / 93$ & 080 & b2 & 2.00 & 330 & 21.40 & 77.9 & 71.0 & $y$ & $n$ & 1 & SF \\
\hline $9 / 2 / 93$ & $08 p$ & b2 & 1.75 & 330 & 21.40 & 77.9 & 67.0 & $y$ & $n$ & 1 & SF \\
\hline $9 / 2 / 93$ & $08 q$ & b2 & 2.00 & 330 & 21.60 & 77.9 & 66.0 & $y$ & $n$ & 1 & \\
\hline $9 / 2 / 93$ & $08 r$ & b2 & 1.75 & 330 & 21.60 & 77.9 & 65.0 & $y$ & $n$ & 1 & US \\
\hline $9 / 6 / 93$ & $08 \mathrm{~s}$ & b2 & 2.00 & 330 & 21.80 & 77.9 & 71.0 & $y$ & $n$ & 1 & US \\
\hline $9 / 6 / 93$ & $08 t$ & b2 & 1.75 & 330 & 21.80 & 77.9 & 69.0 & $y$ & $n$ & 1 & US \\
\hline $9 / 6 / 93$ & $08 u$ & b2 & 2.00 & 330 & 22.00 & 77.9 & 69.0 & $y$ & $\mathrm{n}$ & 1 & US \\
\hline $9 / 6 / 93$ & $08 \mathrm{v}$ & b2 & 1.75 & 330 & 22.00 & 77.9 & 68.0 & $y$ & $n$ & 1 & US \\
\hline $9 / 6 / 93$ & $08 w$ & b2 & 2.00 & 330 & 22.20 & 77.9 & 68.0 & $y$ & $n$ & 0 & US \\
\hline $9 / 6 / 93$ & $08 x$ & b2 & 1.75 & 330 & 22.20 & 77.9 & 68.0 & $y$ & $n$ & 0 & US \\
\hline $9 / 7 / 93$ & $08 y$ & b2 & 2.00 & 330 & 22.40 & 77.9 & 71.0 & $y$ & $n$ & 1 & US \\
\hline $9 / 7 / 93$ & $08 z$ & b2 & 1.75 & 330 & 22.40 & 77.9 & 69.0 & $y$ & $n$ & 1 & US \\
\hline $9 / 7 / 93$ & $09 a$ & b2 & 2.00 & 330 & 22.60 & 77.9 & 68.0 & $y$ & $n$ & 1 & US \\
\hline $9 / 7 / 93$ & $09 b$ & b2 & 1.75 & 330 & 22.60 & 77.9 & 66.0 & $y$ & $n$ & 1 & US \\
\hline $9 / 7 / 93$ & $09 c$ & b2 & 2.00 & 330 & 20.80 & 77.9 & 66.0 & $y$ & $n$ & 1 & SF \\
\hline $9 / 7 / 93$ & $09 d$ & b2 & 1.75 & 330 & 20.80 & 77.9 & 65.0 & $y$ & $n$ & 1 & SF \\
\hline $9 / 8 / 93$ & $09 \mathrm{e}$ & b2 & 2.00 & 330 & 20.60 & 77.9 & 70.0 & $y$ & $n$ & 1 & SF \\
\hline $9 / 8 / 93$ & $09 f$ & b2 & 1.75 & 330 & 20.60 & 77.9 & 67.0 & $y$ & $n$ & 1 & SF \\
\hline $9 / 13 / 93$ & $\overline{09 g}$ & b2 & 2.00 & 330 & 22.80 & 77.9 & 67.0 & $y$ & $n$ & 1 & US \\
\hline $9 / 13 / 93$ & $09 \mathrm{~g}$ & b2 & 2.00 & 330 & 22.80 & 77.9 & 67.0 & $y$ & $n$ & 1 & US \\
\hline $9 / 13 / 93$ & $09 \mathrm{~h}$ & b2 & 1.75 & 330 & 22.80 & 77.9 & 63.0 & $y$ & $n$ & 1 & \\
\hline $9 / 14 / 93$ & $09 i$ & b2 & 2.00 & 330 & 23.00 & 77.9 & 69.0 & $\bar{Y}$ & $\bar{N}$ & 1 & SC \\
\hline $9 / 14 / 93$ & $09 i$ & b2 & 2.00 & 330 & 23.00 & 77.9 & 69.0 & $y$ & $n$ & 1 & SC \\
\hline $9 / 14 / 93$ & $09 j$ & b2 & 1.75 & 330 & 23.00 & 77.9 & 64.0 & $\bar{Y}$ & $\bar{N}$ & 1 & SC \\
\hline $9 / 14 / 93$ & $09 k$ & b2 & 2.00 & 330 & 23.20 & 77.9 & 61.0 & $\bar{Y}$ & $\mathbf{N}$ & 1 & SC \\
\hline $9 / 14 / 93$ & 091 & b2 & 1.75 & 330 & 23.20 & 77.9 & 62.0 & $\bar{Y}$ & $\mathbf{N}$ & 1 & SC \\
\hline $9 / 14 / 93$ & $09 m$ & b2 & 2.00 & 330 & 23.40 & 77.9 & 63.0 & $\bar{Y}$ & $\mathbf{N}$ & 1 & SC \\
\hline $9 / 15 / 93$ & $09 n$ & b2 & 1.75 & 330 & 23.40 & 77.9 & 71.0 & $\bar{Y}$ & $\mathbf{N}$ & 1 & SC \\
\hline $9 / 17 / 93$ & 090 & b2 & 2.00 & 330 & 23.40 & 77.9 & 68.0 & $y$ & $n$ & 1 & SC \\
\hline 9/17/93 & $09 p$ & b2 & 2.00 & 330 & 23.40 & 77.9 & 56.0 & $y$ & $n$ & 1 & SC \\
\hline $9 / 17 / 93$ & $09 q$ & b2 & 2.00 & 330 & 23.40 & 77.9 & 66.0 & $y$ & $n$ & 1 & SC \\
\hline $9 / 20 / 93$ & $09 r$ & b2 & 2.00 & 330 & 23.40 & 77.9 & 68.0 & $y$ & $n$ & $\overline{1}$ & SC \\
\hline $9 / 20 / 93$ & $09 \mathrm{~s}$ & b2 & 2.00 & 330 & 23.40 & 77.9 & 64.0 & $y$ & $y$ & 1 & SC \\
\hline
\end{tabular}


Data Summary

\begin{tabular}{|c|c|c|c|c|c|c|c|c|c|c|c|}
\hline DATE & EXP & BASE & GATE & MTOT & ANGLE & TEMP & HUMID & CAM & FIBER & QUAL & STATE \\
\hline $9 / 20 / 93$ & $09 t$ & b2 & 2.00 & 330 & 23.40 & 77.9 & \begin{tabular}{|l|}
64.0 \\
\end{tabular} & $y$ & $n$ & 1 & SC \\
\hline $9 / 20 / 93$ & $09 u$ & b2 & 2.00 & 330 & 23.40 & 77.9 & 63.0 & $y$ & $n$ & 1 & SC \\
\hline $9 / 21 / 93$ & $09 v$ & b2 & 2.00 & 330 & 23.40 & 77.9 & 70.0 & $y$ & $\mathbf{n}$ & 1 & SC \\
\hline $9 / 21 / 93$ & $09 w$ & b2 & 2.00 & 330 & 23.40 & 77.9 & 66.0 & $y$ & $n$ & 1 & SC \\
\hline $9 / 21 / 93$ & $09 x$ & b2 & 2.00 & 330 & 23.40 & 77.9 & 66.0 & $y$ & $n$ & 1 & SC \\
\hline $9 / 22 / 93$ & $09 y$ & b2 & 2.00 & 330 & 23.40 & 77.9 & 66.0 & & & 1 & SC \\
\hline $9 / 22 / 93$ & $10 a$ & b2 & 2.00 & 330 & 23.40 & 77.9 & 66.0 & & & 1 & SC \\
\hline $9 / 22 / 93$ & $10 \mathrm{~b}$ & b2 & 2.00 & 330 & 23.40 & 77.9 & 65.0 & & & 1 & SC \\
\hline $9 / 22 / 93$ & $10 c$ & b2 & 2.00 & 330 & 23.40 & 77.9 & 65.0 & & & 1 & SC \\
\hline $9 / 23 / 93$ & $10 \mathrm{~d}$ & b2 & 2.00 & 330 & 23.40 & 77.9 & 64.0 & $y$ & $\mathbf{n}$ & 1 & SC \\
\hline $9 / 23 / 93$ & $10 \mathrm{e}$ & $\mathrm{b} 2$ & 2.00 & 330 & 23.40 & 77.9 & 64.0 & $y$ & $n$ & 1 & SC \\
\hline $9 / 23 / 93$ & $10 f$ & b2 & 2.00 & 330 & 23.40 & 77.9 & 64.0 & $y$ & $n$ & 1 & SC \\
\hline $9 / 23 / 93$ & $10 \mathrm{~g}$ & b2 & 2.00 & 330 & 23.40 & 77.9 & 64.0 & $y$ & $n$ & 1 & SC \\
\hline $9 / 23 / 93$ & $10 \mathrm{~h}$ & b2 & 2.00 & 330 & 23.40 & 77.9 & 64.0 & $y$ & $n$ & 1 & SC \\
\hline $9 / 23 / 93$ & $10 i$ & b2 & 2.00 & 330 & 23.40 & 77.9 & 64.0 & $y$ & $n$ & 1 & SC \\
\hline $9 / 24 / 93$ & $10 \mathrm{j}$ & b2 & 2.00 & 330 & 23.40 & 77.9 & 63.0 & $y$ & $y$ & 1 & SC \\
\hline $9 / 24 / 93$ & $10 \mathrm{~K}$ & b2 & 2.00 & 330 & 23.40 & 77.9 & 63.0 & $y$ & $y$ & 1 & SC \\
\hline $9 / 24 / 93$ & 101 & b2 & 2.00 & 330 & 23.40 & 77.9 & 63.0 & $y$ & $y$ & 1 & SC \\
\hline $9 / 24 / 93$ & $10 \mathrm{~m}$ & b2 & 2.00 & 330 & 23.40 & 77.9 & 63.0 & $y$ & $y$ & 1 & SC \\
\hline $10 / 6 / 93$ & $10 n$ & $\mathrm{~b} 2$ & 2.00 & 330 & 23.40 & 77.9 & 63.0 & $y$ & $y$ & 1 & SC \\
\hline $10 / 6 / 93$ & 100 & b2 & 2.00 & 330 & 23.40 & 77.9 & 62.0 & $y$ & $n$ & 1 & SC \\
\hline $10 / 7 / 93$ & $10 p$ & b2 & 2.00 & 330 & 23.40 & 77.9 & 59.0 & $y$ & $y$ & 1 & SC \\
\hline $10 / 11 / 93$ & $10 q$ & b2 & 2.00 & 330 & 23.40 & 77.9 & 58.0 & $y$ & $y$ & 1 & SC \\
\hline $10 / 11 / 93$ & $10 r$ & b2 & 2.00 & 330 & 23.40 & 77.9 & 54.0 & & & 1 & $\overline{\mathrm{sc}}$ \\
\hline $10 / 12 / 93$ & 10s & b2 & 2.00 & 330 & 23.40 & 77.9 & 54.0 & $y$ & $y$ & 1 & SC \\
\hline $10 / 12 / 93$ & $10 t$ & b2 & 2.00 & 330 & 23.40 & 77.9 & 52.0 & $y$ & $y$ & 1 & SC \\
\hline $10 / 12 / 93$ & $10 u$ & b2 & 2.00 & 330 & 23.40 & 77.9 & 49.0 & & & 1 & SC \\
\hline $10 / 13 / 93$ & $10 w$ & b2 & 2.00 & 330 & 23.40 & 77.9 & 48.0 & $y$ & $y$ & 1 & SC \\
\hline $10 / 14 / 93$ & $10 y$ & b2 & 2.00 & 330 & 23.40 & 77.9 & 57.0 & $y$ & $y$ & 1 & SC \\
\hline $10 / 18 / 93$ & $10 z$ & b2 & 2.00 & 330 & 21.20 & 77.9 & 52.0 & $y$ & $y$ & 1 & SF \\
\hline $10 / 18 / 93$ & $11 a$ & b2 & 2.00 & 330 & 21.20 & 77.9 & 56.0 & $y$ & $y$ & 1 & SF \\
\hline $10 / 18 / 93$ & $11 b$ & b2 & 2.00 & 330 & 21.20 & 77.9 & 54.0 & $y$ & $y$ & 1 & SF \\
\hline $10 / 19 / 93$ & $11 \mathrm{c}$ & b2 & 2.00 & 330 & 21.20 & 77.9 & 62.0 & $y$ & $y$ & 1 & SF \\
\hline $10 / 19 / 93$ & 11d & b2 & 2.00 & 330 & 21.20 & 77.9 & 56.0 & $y$ & $y$ & 1 & SF \\
\hline $10 / 19 / 93$ & $11 \mathrm{e}$ & b2 & 2.00 & 330 & 21.20 & 77.9 & 56.0 & $y$ & $y$ & 1 & SF \\
\hline $10 / 19 / 93$ & $11 f$ & b2 & 2.00 & 330 & 21.20 & 77.9 & 54.0 & $y$ & $y$ & 1 & SF \\
\hline $10 / 20 / 93$ & $11 \mathrm{~g}$ & b2 & 2.00 & 330 & 21.20 & 77.9 & 60.0 & $y$ & $y$ & 1 & SF \\
\hline $10 / 20 / 93$ & $11 \mathrm{~h}$ & b2 & 2.00 & 330 & 21.20 & 77.9 & 58.0 & $y$ & $y$ & 1 & SF \\
\hline $10 / 20 / 93$ & $11 i$ & b2 & 2.00 & 330 & 21.20 & 77.9 & 56.0 & $y$ & $y$ & 1 & SF \\
\hline $10 / 21 / 93$ & $11 \mathrm{j}$ & b2 & 2.00 & 330 & 21.20 & 77.9 & 60.0 & $y$ & $y$ & 1 & SF \\
\hline $10 / 21 / 93$ & $11 k$ & b2 & 2.00 & 330 & 21.20 & 77.9 & 59.0 & $y$ & $y$ & 1 & SF \\
\hline $10 / 21 / 93$ & 111 & b2 & 2.00 & 330 & 21.20 & 77.9 & 57.0 & $y$ & $y$ & 1 & SF \\
\hline $10 / 21 / 93$ & $11 \mathrm{~m}$ & b2 & 2.00 & 330 & 21.20 & 77.9 & 57.0 & $y$ & $y$ & 1 & SF \\
\hline $10 / 21 / 93$ & $11 n$ & b2 & 2.00 & 330 & 21.20 & 77.9 & 57.0 & $y$ & $y$ & 1 & SF \\
\hline $10 / 22 / 93$ & $11 p$ & b2 & 2.00 & 330 & 21.20 & 77.9 & 62.0 & $y$ & $y$ & 1 & SF \\
\hline $10 / 22 / 93$ & $11 q$ & b2 & 2.00 & 330 & 21.20 & 77.9 & 59.0 & $y$ & $y$ & 1 & SF \\
\hline $10 / 22 / 93$ & $11 \mathrm{r}$ & b2 & 2.00 & 330 & 21.20 & 77.9 & 58.0 & $y$ & $y$ & 1 & SF \\
\hline $10 / 22 / 93$ & $11 \mathrm{~s}$ & $\mathrm{~b} 2$ & 2.00 & 330 & 21.20 & 77.9 & 56.0 & $Y$ & $Y$ & 1 & SF \\
\hline $10 / 22 / 93$ & $11 t$ & b2 & 2.00 & 330 & 21.20 & 77.9 & 56.0 & $y$ & $y$ & 1 & SF \\
\hline $10 / 25 / 93$ & $11 u$ & b2 & 2.00 & 330 & 21.20 & 77.9 & 58.0 & $y$ & $y$ & 1 & SF \\
\hline
\end{tabular}


Data Summary

\begin{tabular}{|c|c|c|c|c|c|c|c|c|c|c|c|}
\hline DATE & EXP & BASE & GATE & MTOT & ANGLE & TEMP & HUMID & $\overline{C A M}$ & FIBER & QUAL & STATE \\
\hline $10 / 25 / 93$ & $11 \mathrm{v}$ & b2 & 2.00 & 330 & 21.20 & 77.9 & 56.0 & $y$ & $y$ & 1 & SF \\
\hline $10 / 25 / 93$ & $11 w$ & b2 & 2.00 & 330 & 21.20 & 77.9 & 56.0 & $y$ & $y$ & 1 & SF \\
\hline $10 / 25 / 93$ & $11 x$ & b2 & 2.00 & 330 & 21.20 & 77.9 & 55.0 & $y$ & $y$ & 1 & SF \\
\hline $11 / 14 / 93$ & $10 x$ & b2 & 2.00 & 330 & 23.40 & 77.9 & 59.0 & $y$ & $y$ & 1 & SC \\
\hline $11 / 21 / 93$ & 110 & b2 & 2.00 & 330 & 21.20 & 77.9 & 56.0 & $y$ & $y$ & 1 & SF \\
\hline $2 / 23 / 94$ & & b1 & 5.00 & 330 & 23.40 & 0.0 & 61.0 & & & & \\
\hline $2 / 23 / 94$ & & b1 & 5.00 & 330 & 23.40 & 78.0 & 68.0 & & & & \\
\hline $2 / 23 / 94$ & & b1 & 5.00 & 330 & 23.40 & 0.0 & 62.0 & & & & \\
\hline $2 / 24 / 94$ & & b1 & 5.00 & 330 & 23.40 & 0.0 & 62.0 & & & & \\
\hline $4 / 18 / 94$ & $12 a$ & b1 & 0.50 & 330 & 16.00 & 0.0 & 49.0 & $n$ & $n$ & 1 & SF \\
\hline $4 / 18 / 94$ & $12 b$ & b1 & 1.00 & 330 & 16.00 & 0.0 & 49.0 & $n$ & $n$ & 1 & \\
\hline $4 / 18 / 94$ & $12 c$ & b1 & 1.50 & 330 & 16.00 & 0.0 & 49.0 & $n$ & $n$ & 1 & \\
\hline $4 / 18 / 94$ & $12 d$ & b1 & 2.00 & 330 & 16.00 & 0.0 & 49.0 & $n$ & $n$ & 1 & \\
\hline $4 / 18 / 94$ & $12 e$ & b1 & 9.00 & 330 & 16.00 & 78.0 & 49.0 & $n$ & $n$ & 1 & \\
\hline $4 / 18 / 94$ & A12 & b1 & & 100 & 16.00 & 0.0 & 49.0 & & & & \\
\hline $4 / 21 / 94$ & $B 12$ & b1 & & 100 & 16.50 & 78.0 & 60.0 & & & & \\
\hline $5 / 2 / 94$ & $12 f$ & b1 & 0.50 & 330 & 16.50 & 78.0 & 76.0 & & & 1 & nf \\
\hline $5 / 2 / 94$ & $12 \mathrm{~g}$ & b1 & 1.00 & 330 & 16.50 & 78.0 & 76.0 & $n$ & $n$ & 1 & $n f$ \\
\hline $5 / 2 / 94$ & $12 \mathrm{~h}$ & b1 & 1.50 & 330 & 16.50 & 78.0 & 76.0 & $n$ & $n$ & 1 & nf \\
\hline $5 / 2 / 94$ & $12 i$ & b1 & 2.00 & 330 & 16.50 & 75.0 & 76.0 & $n$ & $n$ & 1 & nf \\
\hline $5 / 2 / 94$ & 12] & b1 & 9.00 & 330 & 16.50 & 75.0 & 76.0 & $y$ & $y$ & 1 & \\
\hline $5 / 3 / 94$ & $12 r$ & b1 & 1.50 & 330 & 17.50 & 78.0 & 74.0 & $y$ & $n$ & 1 & \\
\hline $5 / 3 / 94$ & $12 k$ & b1 & 0.50 & 330 & 17.00 & 75.0 & 76.0 & $n$ & $n$ & 1 & nf \\
\hline $5 / 3 / 94$ & 121 & b1 & 1.00 & 330 & 17.00 & 78.0 & 75.0 & $n$ & $n$ & 1 & nf \\
\hline $5 / 3 / 94$ & $12 m$ & b1 & 1.50 & 330 & 17.00 & 78.0 & 75.0 & $\bar{n}$ & $\mathbf{n}$ & 1 & $\overline{n f}$ \\
\hline $5 / 3 / 94$ & $12 n$ & b1 & 2.00 & 330 & 17.00 & 78.0 & 75.0 & $n$ & $\mathrm{n}$ & 1 & $n f$ \\
\hline $5 / 3 / 94$ & 120 & b1 & 9.00 & 330 & 17.00 & 78.0 & 75.0 & $y$ & $y$ & 1 & \\
\hline $5 / 3 / 94$ & $12 p$ & b1 & 0.50 & 330 & 17.50 & 78.0 & 74.0 & $n$ & n & 1 & $n f$ \\
\hline $5 / 3 / 94$ & $12 q$ & b1 & 1.00 & 330 & 17.50 & 78.0 & 74.0 & $n$ & $n$ & 1 & nf \\
\hline $5 / 3 / 94$ & $12 \mathrm{~s}$ & b1 & 2.00 & 330 & 17.50 & 78.0 & 74.0 & $y$ & $y$ & 1 & \\
\hline $5 / 3 / 94$ & $12 t$ & b1 & 9.00 & 330 & 17.50 & 78.0 & 74.0 & $y$ & $y$ & 1 & \\
\hline $5 / 5 / 94$ & $12 u$ & b1 & 0.50 & 330 & 18.00 & 77.0 & 79.0 & n & $n$ & 1 & nf \\
\hline $5 / 5 / 94$ & $12 v$ & b1 & 1.00 & 330 & 18.00 & 78.0 & 79.0 & $y$ & $y$ & 1 & \\
\hline $5 / 6 / 94$ & $12 w$ & b1 & 1.50 & 330 & 18.00 & 78.0 & 79.0 & $y$ & $y$ & 1 & \\
\hline $5 / 6 / 94$ & $12 x$ & $\overline{\mathrm{b} 1}$ & 2.00 & 330 & 18.00 & 77.0 & 57.0 & $y$ & $y$ & 1 & \\
\hline $5 / 6 / 94$ & $12 y$ & b1 & 9.00 & 330 & 18.00 & 77.0 & 57.0 & $y$ & $y$ & 1 & \\
\hline $5 / 6 / 94$ & $12 z$ & b1 & 0.50 & 330 & 18.50 & 77.0 & 52.0 & $y$ & $y$ & 1 & \\
\hline $5 / 6 / 94$ & $13 a$ & b1 & 1.50 & 330 & 18.50 & 78.0 & 51.0 & $y$ & $y$ & 1 & \\
\hline $5 / 6 / 94$ & $13 b$ & b1 & 2.00 & 330 & 18.50 & 77.0 & 51.0 & $y$ & $y$ & 1 & \\
\hline $5 / 6 / 94$ & $13 c$ & b1 & 9.00 & 330 & 18.50 & 77.0 & 51.0 & $y$ & $y$ & 1 & \\
\hline $5 / 9 / 94$ & $13 d$ & b1 & 0.50 & 330 & 19.00 & 75.0 & 67.0 & $n$ & $n$ & 1 & nf \\
\hline $5 / 9 / 94$ & $13 \mathrm{e}$ & b1 & 1.00 & 330 & 19.00 & 77.0 & 67.0 & $y$ & $y$ & 1 & \\
\hline $5 / 9 / 94$ & $13 f$ & b1 & 1.50 & 330 & 19.00 & 77.0 & 65.0 & $y$ & $y$ & 1 & \\
\hline $5 / 9 / 94$ & $13 g$ & b1 & 2.00 & 330 & 19.00 & 77.0 & 63.0 & $y$ & $y$ & 1 & \\
\hline $5 / 9 / 94$ & $13 \mathrm{~h}$ & b1 & 9.00 & 330 & 19.00 & 77.0 & 62.0 & $y$ & $y$ & 1 & \\
\hline $5 / 11 / 94$ & $13 i$ & b1 & 0.50 & 330 & 19.50 & 74.0 & 70.0 & $n$ & $n$ & $T$ & nf \\
\hline $5 / 11 / 94$ & $13 j$ & b1 & 1.00 & 330 & 19.50 & 78.0 & 71.0 & $y$ & $y$ & 1 & \\
\hline $5 / 11 / 94$ & $13 k$ & b1 & 1.50 & 330 & 19.50 & 78.0 & 71.0 & $y$ & $y$ & 1 & \\
\hline $5 / 11 / 94$ & 131 & b1 & 2.00 & 330 & 19.50 & $75: 0$ & 69.0 & $y$ & $y$ & 1 & \\
\hline $5 / 11 / 94$ & $13 m$ & b1 & 9.00 & 330 & 19.50 & 75.0 & 68.0 & $y$ & $y$ & 1 & \\
\hline
\end{tabular}


Data Summary

\begin{tabular}{|c|c|c|c|c|c|c|c|c|c|c|c|}
\hline DATE & EXP & BASE & GATE & MTOT & ANGLE & TEMP & HUMID & CAM & |FIBER & QUAL & STATE \\
\hline $5 / 11 / 94$ & $13 n$ & b1 & 0.50 & 330 & 20.00 & 78.0 & 68.0 & $y$ & \begin{tabular}{|l|}
$y$ \\
\end{tabular} & 1 & \\
\hline $5 / 11 / 94$ & 130 & b1 & 1.00 & 330 & 20.00 & 78.0 & 67.0 & $y$ & $y$ & 1 & \\
\hline $5 / 11 / 94$ & $13 p$ & b1 & 1.50 & 330 & 20.00 & 77.0 & 67.0 & $y$ & $y$ & 1 & \\
\hline $5 / 11 / 94$ & $13 q$ & b1 & 2.00 & 330 & 20.00 & 77.0 & 67.0 & $y$ & $y$ & 1 & \\
\hline $5 / 11 / 94$ & $13 r$ & b1 & 9.00 & 330 & 20.00 & 77.0 & 67.0 & $y$ & $y$ & 1 & \\
\hline $5 / 13 / 94$ & $13 \mathrm{~s}$ & b1 & 9.00 & 330 & 20.50 & 76.0 & 73.0 & $y$ & $y$ & 1 & \\
\hline $5 / 13 / 94$ & $13 t$ & b1 & 2.00 & 330 & 20.50 & 76.0 & 72.0 & $y$ & $y$ & 1 & \\
\hline $5 / 13 / 94$ & $13 u$ & b1 & 1.50 & 330 & 20.50 & 76.0 & 71.0 & $y$ & $y$ & 1 & \\
\hline $5 / 13 / 94$ & $13 v$ & b1 & 1.00 & 330 & 20.50 & 79.0 & 71.0 & $y$ & $y$ & 1 & sf \\
\hline $5 / 13 / 94$ & $13 w$ & b1 & 0.50 & 330 & 20.50 & 77.0 & 69.0 & $y$ & $y$ & 1 & \\
\hline $5 / 13 / 94$ & $13 x$ & b1 & 9.00 & 330 & 21.00 & 78.0 & 69.0 & $y$ & $y$ & 1 & \\
\hline $5 / 13 / 94$ & $13 y$ & b1 & 2.00 & 330 & 21.00 & 78.0 & 69.0 & $y$ & $y$ & 1 & \\
\hline $5 / 13 / 94$ & 132 & b1 & 1.50 & 330 & 21.00 & 78.0 & 64.0 & $y$ & $y$ & 1 & \\
\hline $5 / 16 / 94$ & $14 a$ & b1 & 1.00 & 330 & 21.00 & 78.0 & 74.0 & $y$ & $y$ & 1 & sf \\
\hline $5 / 16 / 94$ & $14 b$ & b1 & 0.50 & 330 & 21.00 & 78.0 & 74.0 & $y$ & $y$ & 1 & \\
\hline $5 / 16 / 94$ & $14 c$ & b1 & 9.00 & 330 & 21.50 & 78.0 & 71.0 & $y$ & $y$ & 1 & \\
\hline $5 / 16 / 94$ & 14d & b1 & 2.00 & 330 & 21.50 & 78.0 & 71.0 & $y$ & $n$ & 1 & sf \\
\hline $5 / 16 / 94$ & $14 e$ & b1 & 1.50 & 330 & 21.50 & 78.0 & 71.0 & $y$ & $n$ & 1 & \\
\hline $5 / 16 / 94$ & $14 f$ & b1 & 1.00 & 330 & 21.50 & 78.0 & 70.0 & $y$ & $n$ & 1 & sf \\
\hline $5 / 17 / 94$ & $14 g$ & b1 & 0.50 & 330 & 21.50 & 78.0 & 74.0 & $y$ & $n$ & 1 & \\
\hline $5 / 17 / 94$ & $14 \mathrm{~h}$ & b1 & 9.00 & 330 & 22.00 & 78.0 & 73.0 & $y$ & $n$ & 1 & \\
\hline $5 / 17 / 94$ & $14 i$ & b1 & 2.00 & 330 & 22.00 & 78.0 & 72.0 & $y$ & $n$ & 1 & \\
\hline $5 / 17 / 94$ & $14 j$ & b1 & 1.50 & 330 & 22.00 & 78.0 & 72.0 & $y$ & $\frac{\pi}{n}$ & 1 & \\
\hline $5 / 17 / 94$ & $14 k$ & b1 & 1.00 & 330 & 22.00 & 78.0 & 72.0 & $y$ & $n$ & 1 & \\
\hline $5 / 17 / 94$ & $14 !$ & b1 & 0.50 & 330 & 22.00 & 78.0 & 72.0 & $y$ & $n$ & 1 & ef \\
\hline $5 / 17 / 94$ & $14 m$ & b1 & 9.00 & 330 & 22.50 & 78.0 & 71.0 & $y$ & $n$ & 1 & \\
\hline $5 / 20 / 94$ & $14 n$ & b1 & 2.00 & 330 & 22.50 & 78.0 & 53.0 & $y$ & $n$ & $\overline{1}$ & sf \\
\hline $5 / 20 / 94$ & 140 & b1 & 1.50 & 330 & 22.50 & 78.0 & 53.0 & $y$ & $n$ & 1 & \\
\hline $5 / 20 / 94$ & $14 p$ & b1 & 1.00 & 330 & 22.50 & 78.0 & 54.0 & $y$ & $n$ & 1 & \\
\hline $5 / 20 / 94$ & $14 q$ & b1 & 0.50 & 330 & 22.50 & 78.0 & 54.0 & $y$ & in & 1 & \\
\hline $5 / 20 / 94$ & $14 r$ & b1 & 9.00 & 330 & 23.00 & 78.0 & 59.0 & $y$ & $n$ & 1 & \\
\hline $5 / 20 / 94$ & $14 \mathrm{~s}$ & b1 & 2.00 & 330 & 23.00 & 78.0 & 53.0 & $y$ & $n$ & 1 & sf \\
\hline $5 / 20 / 94$ & $14 t$ & b1 & 1.00 & 330 & 23.00 & 78.0 & 53.0 & $y$ & $n$ & 1 & sf \\
\hline $5 / 23 / 94$ & $14 u$ & b1 & 0.50 & 330 & 23.00 & 78.0 & 57.0 & $y$ & $n$ & 1 & ef \\
\hline $5 / 23 / 94$ & $14 \mathrm{~V}$ & b1 & 9.00 & 330 & 23.50 & 78.0 & 54.0 & $y$ & $n$ & 1 & \\
\hline $5 / 23 / 94$ & $14 w$ & b1 & 2.00 & 330 & 23.50 & 78.0 & 53.0 & $y$ & $n$ & 1 & \\
\hline $5 / 23 / 94$ & $14 x$ & b1 & 1.50 & 330 & 23.50 & 78.0 & 51.0 & $y$ & $n$ & 1 & ef \\
\hline $5 / 23 / 94$ & $14 y$ & b1 & 1.00 & 330 & 23.50 & 78.0 & 53.0 & $y$ & $n$ & 1 & ef \\
\hline $5 / 23 / 94$ & 142 & b1 & 0.50 & 330 & 23.50 & 78.0 & 53.0 & $y$ & $n$ & 1 & ef \\
\hline $5 / 27 / 94$ & $15 a$ & b1 & 9.00 & 330 & 24.00 & 78.0 & 63.0 & $y$ & $n$ & 1 & ef \\
\hline $5 / 27 / 94$ & $15 b$ & b1 & 2.00 & 330 & 24.00 & 78.0 & 70.0 & $y$ & $n$ & 1 & ef \\
\hline $5 / 30 / 94$ & $15 c$ & b1 & 1.50 & 330 & 24.00 & 78.0 & 75.0 & $y$ & $n$ & 1 & ef \\
\hline $5 / 30 / 94$ & $15 d$ & b1 & 1.00 & 330 & 24.00 & 78.0 & 75.0 & $y$ & $n$ & 1 & ef \\
\hline $5 / 30 / 94$ & $15 e$ & b1 & 0.50 & 330 & 24.00 & 78.0 & 74.0 & $y$ & $n$ & 1 & ef \\
\hline $5 / 30 / 94$ & $15 f$ & b1 & 9.00 & 330 & 24.50 & 78.0 & 74.0 & $y$ & $n$ & 1 & ef \\
\hline $5 / 30 / 94$ & $15 g$ & b1 & 2.00 & 330 & 24.50 & 78.0 & 74.0 & $y$ & $\ddot{n}$ & 1 & ef \\
\hline $5 / 30 / 94$ & $15 \mathrm{~h}$ & b1 & 1.50 & 330 & 24.50 & 78.0 & 74.0 & $y$ & $n$ & 1 & ef \\
\hline $5 / 30 / 94$ & $15 i$ & b1 & 1.00 & 330 & 24.50 & 78.0 & 74.0 & $y$ & $n$ & 1 & ef \\
\hline $8 / 16 / 94$ & $17 d$ & b1 & 5.00 & 330 & 22.50 & 73.0 & 71.0 & $y$ & & & \\
\hline $8 / 16 / 94$ & $17 \mathrm{e}$ & b1 & 5.00 & 330 & 22.50 & 73.0 & 71.0 & $y$ & & & \\
\hline
\end{tabular}


Data Summary

\begin{tabular}{|c|c|c|c|c|c|c|c|c|c|c|c|}
\hline DATE & EXP & BASE & GATE & MTOT & ANGLE & TEMP & HUMID & CAM & FIBER & QUAL & STATE \\
\hline $8 / 17 / 94$ & $17 f$ & b1 & 5.00 & 330 & 22.00 & 74.0 & 68.0 & $y$ & & & \\
\hline $8 / 17 / 94$ & $17 g$ & b1 & 5.00 & 330 & 22.00 & 74.0 & 68.0 & $y$ & & & \\
\hline $8 / 18 / 94$ & $17 \mathrm{~h}$ & b1 & 2.00 & 330 & 21.50 & 74.0 & 71.0 & $y$ & $\mathbf{n}$ & & $?$ \\
\hline $8 / 18 / 94$ & $17 j$ & b1 & 5.00 & 330 & 21.00 & 75.0 & 61.0 & $y$ & & & \\
\hline $8 / 18 / 94$ & $17 \mathrm{k}$ & b1 & 5.00 & 330 & 21.00 & 75.0 & 69.0 & $y$ & & & \\
\hline $9 / 8 / 94$ & 171 & b1 & 5.00 & 330 & 21.00 & 74.0 & 68.0 & & $y$ & & \\
\hline $9 / 9 / 94$ & $17 \mathrm{~m}$ & b1 & & 330 & 23.00 & 78.0 & 69.0 & $n$ & $n$ & & st \\
\hline $9 / 12 / 94$ & $17 n$ & b1 & & 330 & 23.00 & 74.0 & 70.0 & $n$ & $n$ & & st \\
\hline $9 / 12 / 94$ & 170 & b1 & & 330 & 23.00 & 74.0 & 67.0 & $n$ & $n$ & & st \\
\hline $9 / 12 / 94$ & $17 p$ & b1 & & 330 & 23.00 & 75.0 & 07.0 & $n$ & $n$ & & st \\
\hline $9 / 12 / 94$ & $17 q$ & b1 & & 330 & 23.00 & 75.0 & 67.0 & $\mathbf{n}$ & $n$ & & st \\
\hline $9 / 13 / 94$ & $17 r$ & b1 & & 330 & 23.00 & 74.0 & 68.0 & $n$ & $n$ & & st \\
\hline $9 / 13 / 94$ & $17 \mathrm{~s}$ & b1 & & 330 & 23.00 & 75.0 & 68.0 & $n$ & $n$ & & vi \\
\hline $9 / 13 / 94$ & $17 t$ & b1 & 0.00 & 330 & 23.00 & 75.0 & 67.0 & $n$ & $n$ & & st \\
\hline 9/14/94 & $17 u$ & b1 & & 330 & 23.00 & 74.0 & 68.0 & $n$ & $n$ & & st \\
\hline $9 / 14 / 94$ & $17 v$ & b1 & & 330 & 23.00 & 74.0 & 68.0 & $n$ & $n$ & & st \\
\hline $9 / 14 / 94$ & $17 w$ & b1 & & 330 & 23.00 & 74.0 & 68.0 & $n$ & $n$ & & st \\
\hline $9 / 14 / 94$ & $17 x$ & b1 & & 330 & 23.00 & 76.0 & 69.0 & $n$ & $n$ & & st \\
\hline $9 / 14 / 94$ & $17 y$ & b1 & & 330 & 23.00 & 76.0 & 69.0 & $n$ & $n$ & & st \\
\hline $9 / 14 / 94$ & 172 & b1 & & 330 & 23.00 & 76.0 & 68.0 & $n$ & $n$ & & st \\
\hline $9 / 14 / 94$ & $18 a$ & b1 & & 330 & 23.00 & 76.0 & 67.0 & $n$ & $n$ & & st \\
\hline $9 / 14 / 94$ & $18 b$ & b1 & & 330 & 23.00 & 76.0 & 67.0 & $n$ & $n$ & & st \\
\hline $9 / 14 / 94$ & $18 \mathrm{c}$ & b1 & & 330 & 23.00 & 76.0 & 67.0 & $n$ & $n$ & & st \\
\hline $9 / 14 / 94$ & $18 d$ & b1 & 0.00 & 330 & 23.00 & 76.0 & 67.0 & & & & \\
\hline $9 / 25 / 94$ & & b1 & 5.00 & 330 & 23.00 & 74.0 & 62.0 & $y$ & & & \\
\hline $10 / 6 / 94$ & 18k & b1 & 5.00 & 350 & 24.00 & 75.0 & 62.0 & $y$ & & & \\
\hline $10 / 6 / 94$ & 181 & b1 & 5.00 & 350 & 26.00 & 75.0 & 64.0 & & & & \\
\hline $10 / 7 / 94$ & $18 m$ & b1 & 5.00 & 350 & 27.40 & 76.0 & 67.0 & $y$ & & & \\
\hline $10 / 7 / 94$ & $18 n$ & b1 & 5.00 & 350 & 27.40 & 76.0 & 67.0 & $y$ & & & \\
\hline $10 / 10 / 94$ & 180 & b1 & & 350 & 27.10 & 78.0 & 72.0 & $y$ & & & \\
\hline $10 / 10 / 94$ & $18 p$ & b1 & & 350 & 27.10 & 78.0 & 72.0 & $y$ & & & \\
\hline $10 / 10 / 94$ & & b1 & 5.00 & 350 & 27.40 & 74.0 & 70.0 & $n$ & $\mathbf{n}$ & & \\
\hline $10 / 10 / 94$ & & b1 & 5.00 & 350 & 24.60 & 74.0 & 68.0 & $n$ & $n$ & & \\
\hline $10 / 11 / 94$ & & b1 & & 350 & 27.10 & 77.0 & 72.0 & & & & \\
\hline $10 / 14 / 94$ & $18 q$ & b1 & 5.00 & 350 & 27.40 & 76.0 & 72.0 & & $y$ & & \\
\hline $10 / 25 / 94$ & $18 \mathrm{~s}$ & b1 & 5.00 & 350 & 24.00 & 74.0 & 67.0 & & $y$ & & \\
\hline $10 / 26 / 94$ & $18 t$ & b1 & 5.00 & 350 & 27.10 & 75.0 & 64.0 & & $y$ & & \\
\hline
\end{tabular}


Data Summary

\begin{tabular}{|c|c|c|c|c|c|c|c|c|c|c|c|c|c|}
\hline DATE & EXP & $\overline{\mathrm{AH} 2}$ & $\overline{\mathrm{AH} 3}$ & $\overline{\mathrm{AH} 4}$ & $\overline{\mathrm{AH5}}$ & $\overline{\mathrm{AH}}$ & $\mathrm{AH7}$ & S2W & S3W & $S 4 W$ & S5W & S6W & S7W \\
\hline $1 / 19 / 93$ & $\mathrm{OOb}$ & 0.88 & 0.80 & 0.68 & 0.55 & 0.63 & 0.65 & 1.100 & 0.785 & 0.650 & 0.565 & 0.475 & 0.430 \\
\hline $1 / 19 / 93$ & $00 \mathrm{c}$ & 1.58 & 1.28 & 1.13 & 0.95 & 0.93 & 0.83 & 1.610 & 1.290 & 1.200 & 0.850 & 0.750 & 0.680 \\
\hline $1 / 19 / 93$ & ood & 2.15 & 1.83 & 1.60 & 1.38 & 1.23 & 1.18 & 2.625 & 2.125 & 1.773 & 1.565 & 1.315 & 1.242 \\
\hline $1 / 19 / 93$ & $\mathrm{OOe}$ & 2.75 & 2.15 & 2.08 & 1.93 & 1.85 & 1.70 & 2.705 & 2.435 & 2.070 & 1.905 & 1.725 & 1.680 \\
\hline $1 / 19 / 93$ & $00 f$ & 0.00 & 0.00 & 0.00 & 0.00 & 0.00 & 0.00 & 0.000 & 0.000 & 0.000 & 0.000 & 0.000 & 0.000 \\
\hline $1 / 21 / 93$ & $00 \mathrm{~g}$ & 0.50 & 0.50 & 0.58 & 0.60 & 0.70 & 0.80 & 0.304 & 0.239 & 0.192 & 0.179 & 0.154 & 0.152 \\
\hline $1 / 21 / 93$ & ooh & 0.44 & 0.47 & 0.70 & 0.72 & 0.71 & 0.77 & 0.285 & 0.190 & 0.160 & 0.137 & 0.077 & 0.167 \\
\hline $1 / 25 / 93$ & $00 \mathrm{i}$ & 2.66 & 2.29 & 2.20 & 2.07 & 1.99 & 1.86 & 2.705 & 2.435 & 2.070 & 1.905 & 1.725 & 1.680 \\
\hline $1 / 27 / 93$ & $00 \mathrm{j}$ & 2.18 & 1.83 & 1.57 & 1.38 & 1.24 & 1.15 & 2.318 & 1.887 & 1.610 & 1.408 & 1.312 & 1.162 \\
\hline $1 / 29 / 93$ & OOK & 1.57 & 1.28 & 1.09 & 0.97 & 0.90 & 0.80 & 1.610 & 1.290 & 1.200 & 0.850 & 0.750 & 0.680 \\
\hline $1 / 31 / 93$ & 001 & 0.56 & 0.56 & 0.63 & 0.67 & 0.75 & 0.79 & 0.303 & 0.226 & 0.181 & 0.169 & 0.151 & 0.149 \\
\hline $1 / 31 / 93$ & $00 \mathrm{~m}$ & 0.88 & 0.79 & 0.68 & 0.64 & 0.62 & 0.61 & 0.960 & 0.723 & 0.582 & 0.510 & 0.465 & 0.423 \\
\hline $2 / 10 / 93$ & $00 n$ & 0.50 & 0.55 & 0.58 & 0.67 & 0.73 & 0.75 & 0.325 & 0.272 & 0.221 & 0.207 & 0.179 & 0.176 \\
\hline $2 / 10 / 93$ & 000 & 0.50 & 0.54 & 0.59 & 0.64 & 0.72 & 0.74 & 0.324 & 0.271 & 0.220 & 0.201 & 0.176 & 0.170 \\
\hline $2 / 11 / 93$ & $00 p$ & 1.55 & 1.08 & 0.85 & 0.77 & 0.71 & 0.64 & 1.310 & 1.026 & 0.785 & 0.687 & 0.656 & 0.523 \\
\hline $2 / 11 / 93$ & $00 \mathrm{q}$ & 1.55 & 1.08 & 0.85 & 0.77 & 0.70 & 0.65 & 1.292 & 1.031 & 0.785 & 0.723 & 0.620 & 0.543 \\
\hline $2 / 11 / 93$ & $00 r$ & 2.53 & 1.61 & 0.89 & 0.70 & 0.69 & 0.63 & 2.430 & 2.230 & 1.759 & 1.595 & 1.387 & 1.228 \\
\hline $2 / 12 / 93$ & $00 \mathrm{~s}$ & 3.13 & 1.69 & 0.94 & 0.73 & 0.63 & 0.55 & 3.133 & 2.913 & 2.373 & 2.203 & 1.943 & 1.758 \\
\hline $2 / 12 / 93$ & $00 t$ & 2.02 & 1.94 & 1.80 & 1.72 & 1.65 & 1.52 & 3.080 & 3.130 & 2.773 & 2.728 & 2.618 & 2.460 \\
\hline $2 / 12 / 93$ & $00 u$ & 1.80 & 2.11 & 1.95 & 1.92 & 1.83 & 1.70 & 3.080 & 3.130 & 2.773 & 2.728 & 2.618 & 2.460 \\
\hline $2 / 23 / 93$ & $\overline{00 v}$ & 4.60 & 4.40 & 4.40 & 4.40 & 4.40 & 4.40 & 4.510 & 4.176 & 3.826 & 3.883 & 4.207 & 3.923 \\
\hline $2 / 24 / 93$ & $00 w$ & 1.50 & 1.45 & 1.40 & 1.40 & 1.40 & 1.40 & 1.075 & 1.060 & 1.000 & 1.095 & 1.140 & 1.037 \\
\hline $2 / 24 / 93$ & $00 x$ & 2.30 & 2.30 & 2.30 & 2.30 & 2.30 & 2.30 & 1.795 & 1.690 & 1.545 & 1.570 & 1.610 & 1.500 \\
\hline $2 / 24 / 93$ & $00 y$ & 2.95 & 3.00 & 3.00 & 3.00 & 3.00 & 3.00 & 2.465 & 2.375 & 2.260 & 2.240 & 2.325 & 2.170 \\
\hline $2 / 24 / 93$ & $00 z$ & 3.30 & 3.60 & 3.60 & 3.70 & 3.70 & 3.70 & 3.030 & 2.940 & 2.750 & 2.775 & 2.885 & 2.675 \\
\hline $3 / 2 / 93$ & $01 a$ & 4.75 & 9.00 & 13.00 & 18.50 & 23.00 & 23.50 & 0.338 & 0.235 & 0.187 & 0.176 & 0.174 & 0.159 \\
\hline $3 / 2 / 93$ & $01 \mathrm{~b}$ & 2.90 & 3.65 & 5.40 & 9.00 & 10.75 & 13.25 & 1.420 & 1.000 & 0.740 & 0.640 & 0.610 & 0.520 \\
\hline $3 / 2 / 93$ & $01 c$ & 3.30 & 3.50 & 3.65 & 3.90 & 5.10 & 6.90 & 2.250 & 1.960 & 1.580 & 1.320 & 1.200 & 0.980 \\
\hline $3 / 2 / 93$ & 01d & 3.45 & 3.80 & 4.00 & 4.70 & 4.85 & 4.85 & 2.900 & 2.640 & 2.260 & 2.100 & 1.980 & 1.670 \\
\hline $3 / 2 / 93$ & $\overline{01 e}$ & 4.20 & 4.80 & 4.80 & 4.80 & 4.90 & 5.00 & 4.560 & 4.380 & 3.900 & 3.820 & 3.980 & 3.600 \\
\hline $3 / 2 / 93$ & 019 & 3.55 & 4.70 & 5.60 & 7.75 & 8.80 & 10.50 & 1.580 & 1.280 & 1.050 & 0.940 & 0.910 & 0.800 \\
\hline $3 / 3 / 93$ & $01 \mathrm{~h}$ & 4.20 & 4.20 & 4.45 & 4.95 & 5.20 & 5.75 & 2.520 & 2.310 & 2.000 & 1.855 & 1.770 & 1.510 \\
\hline $3 / 3 / 93$ & $01 f$ & 7.00 & 9.75 & 13.00 & 15.50 & 18.75 & 20.50 & 0.420 & 0.385 & 0.328 & 0.318 & 0.329 & 0.280 \\
\hline $3 / 4 / 93$ & $01 k$ & 3.35 & 3.45 & 3.45 & 3.45 & 3.45 & 3.45 & 3.100 & 2.890 & 2.880 & 3.120 & 3.310 & 2.940 \\
\hline $3 / 9 / 93$ & $01 i$ & 2.25 & 2.25 & 2.35 & 2.35 & 2.35 & 2.35 & 1.940 & 1.870 & 1.975 & 1.770 & 1.680 & 2.050 \\
\hline $3 / 9 / 93$ & $01 j$ & 2.65 & 2.90 & 2.90 & 2.90 & 2.90 & 2.90 & 2.390 & 2.590 & 2.400 & 2.220 & 2.610 & 2.440 \\
\hline $3 / 9 / 93$ & 011 & 4.45 & 4.45 & 0.00 & 4.45 & 0.00 & 4.55 & 4.970 & 4.280 & 3.800 & 4.020 & 4.040 & 4.340 \\
\hline $3 / 9 / 93$ & $01 \mathrm{~m}$ & 6.30 & 7.50 & 8.05 & 8.65 & 9.90 & 11.40 & 0.490 & 0.420 & 0.380 & 0.380 & 0.410 & 0.340 \\
\hline $3 / 9 / 93$ & $01 n$ & 3.20 & 2.75 & 2.55 & 2.55 & 2.55 & 2.55 & 2.060 & 2.240 & 1.960 & 1.940 & 2.660 & 1.960 \\
\hline $3 / 9 / 93$ & 010 & 4.00 & 4.00 & 4.00 & 4.00 & 4.00 & 4.00 & 3.240 & 3.220 & 2.580 & 2.780 & 2.880 & 3.260 \\
\hline $3 / 15 / 93$ & $01 p$ & 6.20 & 10.25 & 16.50 & 24.50 & 26.00 & 27.50 & 0.254 & 0.186 & 0.164 & 0.140 & 0.146 & 0.135 \\
\hline $3 / 15 / 93$ & 019 & 2.75 & 4.50 & 7.00 & 9.00 & 12.25 & 15.50 & 1.120 & 0.710 & 0.550 & 0.510 & 0.480 & 0.440 \\
\hline $3 / 15 / 93$ & $01 r$ & 3.35 & 3.65 & 4.05 & 6.35 & 7.50 & 9.00 & 2.000 & 1.600 & 1.190 & 1.000 & 0.920 & 0.790 \\
\hline $3 / 15 / 93$ & 015 & 3.30 & 3.45 & 3.45 & 3.85 & 4.65 & 5.50 & 2.900 & 2.400 & 1.940 & 1.720 & 1.580 & 1.270 \\
\hline $3 / 15 / 93$ & $01 t$ & 4.30 & 4.30 & 4.30 & 4.30 & 4.40 & 4.40 & 3.940 & 3.620 & 3.220 & 3.180 & 3.140 & 2.880 \\
\hline $3 / 15 / 93$ & $01 \mathrm{u}$ & 6.85 & 9.40 & 15.10 & 16.30 & 18.00 & 21.00 & 0.440 & 0.387 & 0.326 & 0.313 & 0.316 & 0.260 \\
\hline $3 / 15 / 93$ & $01 \mathrm{v}$ & 3.70 & 4.30 & 5.15 & 6.65 & 8.25 & 10.25 & 1.520 & 1.180 & 0.900 & 0.800 & 0.760 & 0.680 \\
\hline $3 / 15 / 93$ & $01 w$ & 3.95 & 4.30 & 4.50 & 5.35 & 6.20 & 7.25 & 2.040 & 1.760 & 1.420 & 1.280 & 1.200 & 1.140 \\
\hline $3 / 18 / 93$ & $01 x$ & 3.10 & 3.00 & 2.80 & 3.00 & 3.00 & 2.90 & 3.500 & 3.140 & 2.890 & 2.880 & 3.150 & 2.650 \\
\hline $3 / 18 / 93$ & $01 y$ & 0.00 & 0.00 & 0.00 & 0.00 & 0.00 & 0.00 & 0.000 & 0.000 & 0.000 & 0.000 & 0.000 & 0.000 \\
\hline
\end{tabular}




\begin{tabular}{|c|c|c|c|c|c|c|c|c|c|c|c|c|c|}
\hline DATE & EXP & $\mathrm{AH} 2$ & $\mathrm{AH3}$ & $\mathrm{AH} 4$ & AH5 & $\mathrm{AH} 6$ & $\mathrm{AH7}$ & S2W & S3W & S4W & S5W & S6W & S7W \\
\hline $3 / 18 / 93$ & 012 & 4.50 & 3.40 & 3.20 & 3.40 & 3.40 & 3.30 & 4.920 & 3.900 & 3.510 & 3.480 & 3.660 & 3.140 \\
\hline $3 / 18 / 93$ & $02 a$ & 3.00 & 2.80 & 3.00 & 3.00 & 3.00 & 3.00 & 3.220 & 3.305 & 3.340 & 3.160 & 3.080 & 2.440 \\
\hline $3 / 18 / 93$ & $02 b$ & 3.20 & 3.10 & 3.00 & 3.30 & 3.30 & 3.00 & 3.810 & 3.420 & 3.160 & 3.290 & 3.600 & 3.210 \\
\hline $3 / 18 / 93$ & $02 c$ & 5.80 & 4.40 & 4.00 & 4.00 & 4.00 & 4.00 & 6.680 & 5.240 & 4.690 & 4.440 & 4.760 & 4.490 \\
\hline $3 / 18 / 93$ & 02d & 4.30 & 4.00 & 3.60 & 3.60 & 3.60 & 3.60 & 5.160 & 4.500 & 3.880 & 3.920 & 4.140 & 3.620 \\
\hline $3 / 19 / 93$ & $02 e$ & 5.80 & 4.50 & 4.30 & 4.30 & 4.20 & 4.00 & 6.400 & 5.100 & 4.520 & 4.420 & 4.840 & 4.300 \\
\hline $3 / 22 / 93$ & $02 f$ & 10.00 & 16.75 & 23.00 & 30.00 & 34.00 & 37.50 & 0.400 & 0.329 & 0.272 & 0.255 & 0.265 & 0.246 \\
\hline $3 / 22 / 93$ & 029 & 4.65 & 9.75 & 13.75 & 18.00 & 26.25 & 29.50 & 1.060 & 0.800 & 0.650 & 0.600 & 0.590 & 0.520 \\
\hline $3 / 22 / 93$ & $02 h$ & 4.40 & 5.10 & 6.35 & 8.10 & 13.00 & 16.00 & 1.740 & 1.400 & 1.130 & 1.025 & 1.020 & 0.910 \\
\hline $3 / 22 / 93$ & $02 i$ & 10.25 & 19.50 & 25.50 & 32.00 & 39.00 & 43.50 & 0.217 & 0.173 & 0.143 & 0.142 & 0.149 & 0.132 \\
\hline $3 / 22 / 93$ & $09 z$ & 0.00 & 0.00 & 0.00 & 0.00 & 0.00 & 0.00 & 0.000 & 0.000 & 0.000 & 0.000 & 0.000 & 0.000 \\
\hline $3 / 23 / 93$ & 02j & 6.00 & 11.50 & 13.75 & 24.00 & 29.50 & 32.50 & 0.620 & 0.490 & 0.390 & 0.370 & 0.370 & 0.340 \\
\hline $3 / 23 / 93$ & 02k & 3.45 & 6.85 & 9.25 & 13.50 & 16.50 & 26.50 & 1.180 & 0.910 & 0.740 & 0.670 & 0.680 & 0.600 \\
\hline $3 / 23 / 93$ & 021 & 3.65 & 4.40 & 5.55 & 9.60 & 12.80 & 18.15 & 1.870 & 1.450 & 1.120 & 1.010 & 1.010 & 0.910 \\
\hline $3 / 23 / 93$ & $02 m$ & 4.25 & 4.50 & 5.35 & 6.30 & 7.00 & 7.70 & 2.485 & 2.033 & 1.650 & 1.530 & 1.500 & 1.335 \\
\hline $3 / 24 / 93$ & $02 n$ & 10.25 & 15.25 & 22.25 & 28.75 & 37.50 & 38.00 & 0.350 & 0.287 & 0.238 & $\begin{array}{l}0.227 \\
\end{array}$ & 0.237 & 0.209 \\
\hline $3 / 24 / 93$ & 020 & 4.55 & 8.90 & 11.50 & 16.00 & 21.50 & 26.75 & 0.880 & 0.700 & 0.590 & 0.550 & 0.550 & 0.490 \\
\hline $3 / 24 / 93$ & $02 p$ & 4.15 & 4.65 & 5.95 & 7.15 & 9.70 & 13.75 & 1.890 & 1.510 & 1.220 & 1.130 & 1.120 & 0.960 \\
\hline $3 / 24 / 93$ & $02 q$ & 9.50 & 17.25 & 26.50 & 32.50 & 37.00 & 37.50 & 0.244 & 0.186 & 0.152 & 0.147 & 0.154 & 0.136 \\
\hline $3 / 24 / 93$ & $02 r$ & 5.15 & 8.80 & 15.10 & 21.00 & 30.75 & 28.75 & 0.760 & 0.550 & 0.450 & 0.410 & 0.420 & 0.400 \\
\hline $3 / 24 / 93$ & $02 \mathrm{~s}$ & 3.50 & 5.50 & 8.35 & 12.00 & 15.60 & 20.10 & 1.390 & 1.030 & 0.830 & 0.760 & 0.750 & 0.660 \\
\hline $3 / 24 / 93$ & $02 t$ & 3.55 & 4.05 & 4.65 & 7.25 & 9.90 & 12.50 & 2.140 & 1.620 & 1.280 & 1.130 & 1.120 & 0.960 \\
\hline $3 / 24 / 93$ & $02 u$ & 4.25 & 4.65 & 4.65 & 5.80 & 6.90 & 6.90 & 3.000 & 2.520 & 2.040 & 1.880 & 1.840 & 1.560 \\
\hline $3 / 24 / 93$ & $02 v$ & 7.80 & 19.15 & 24.00 & 30.50 & 38.50 & 40.00 & 0.239 & 0.185 & 0.152 & 0.145 & 0.143 & 0.128 \\
\hline $3 / 24 / 93$ & $02 w$ & 4.75 & 8.10 & 15.60 & 22.25 & 33.50 & 40.00 & 0.710 & 0.540 & 0.450 & 0.410 & 0.410 & 0.373 \\
\hline $3 / 25 / 93$ & $02 x$ & 3.65 & 5.90 & 8.90 & 11.35 & 14.50 & 18.75 & 1.340 & 1.000 & 0.820 & 0.740 & 0.740 & 0.620 \\
\hline $3 / 25 / 93$ & $02 y$ & 3.35 & 4.45 & 4.95 & 7.15 & 10.00 & 13.20 & 2.080 & 1.550 & 1.220 & 1.060 & 1.050 & 0.920 \\
\hline $3 / 25 / 93$ & $02 z$ & 4.15 & 4.55 & 5.05 & 6.40 & 6.65 & 7.00 & 2.840 & 2.340 & 1.910 & 1.740 & 1.690 & 1.440 \\
\hline $3 / 25 / 93$ & $\overline{03 a}$ & 10.20 & 16.25 & 23.20 & 31.00 & 36.00 & 39.50 & 0.364 & 0.278 & 0.237 & 0.220 & 0.224 & 0.220 \\
\hline $3 / 25 / 93$ & $03 b$ & 5.55 & 9.80 & 14.00 & 19.50 & 27.50 & 33.00 & 0.820 & 0.680 & 0.580 & 0.520 & 0.540 & 0.440 \\
\hline $3 / 25 / 93$ & $03 c$ & 3.95 & 4.80 & 5.95 & 8.40 & 11.15 & 12.15 & 1.950 & 1.530 & 1.240 & 1.140 & 1.140 & 1.000 \\
\hline $3 / 25 / 93$ & 03d & 7.75 & 13.45 & 16.60 & 24.75 & 34.25 & 34.75 & 0.205 & 0.150 & 0.115 & 0.110 & 0.105 & 0.095 \\
\hline $3 / 25 / 93$ & $03 \mathrm{e}$ & 3.80 & 8.30 & 10.70 & 15.15 & 19.25 & 24.40 & 0.807 & 0.545 & 0.440 & 0.405 & 0.400 & 0.353 \\
\hline $3 / 25 / 93$ & $03 f$ & 3.00 & 4.00 & 5.05 & 8.25 & 10.00 & 12.25 & 1.567 & 1.110 & 0.847 & 0.750 & 0.740 & 0.640 \\
\hline $3 / 25 / 93$ & $03 \mathrm{~g}$ & 3.30 & 3.85 & 4.00 & 5.60 & 6.80 & 7.90 & 2.340 & 1.817 & 1.371 & 1.193 & 1.130 & 0.967 \\
\hline $3 / 25 / 93$ & $03 \mathrm{~h}$ & 3.95 & 4.00 & 4.35 & 4.80 & 5.30 & 5.30 & 3.295 & 2.880 & 2.385 & 2.170 & 2.087 & 1.775 \\
\hline $3 / 26 / 93$ & $03 i$ & 8.75 & 13.90 & 17.80 & 23.50 & 30.50 & 36.00 & 0.340 & 0.280 & 0.233 & 0.230 & 0.235 & 0.215 \\
\hline $3 / 26 / 93$ & $03 j$ & 5.00 & 8.25 & 9.45 & 12.75 & 14.35 & 19.50 & 0.860 & 0.705 & 0.580 & 0.560 & 0.560 & 0.515 \\
\hline $3 / 26 / 93$ & $03 k$ & 3.85 & 4.40 & 4.70 & 6.30 & 8.45 & 10.00 & 1.970 & 1.595 & 1.285 & 1.170 & 1.155 & 1.025 \\
\hline $3 / 26 / 93$ & 031 & 5.60 & 9.80 & 14.90 & 18.50 & 22.00 & 27.00 & 0.245 & 0.177 & 0.143 & 0.130 & 0.125 & 0.115 \\
\hline $3 / 26 / 93$ & $03 m$ & 2.60 & 3.70 & 5.35 & 7.50 & 9.10 & 13.25 & 1.230 & 0.785 & 0.560 & 0.485 & 0.477 & 0.420 \\
\hline $3 / 26 / 93$ & $03 n$ & 3.00 & 3.25 & 3.55 & 4.10 & 4.50 & 5.80 & 2.065 & 1.750 & 1.345 & 1.120 & 0.990 & 0.835 \\
\hline $3 / 26 / 93$ & 030 & 3.10 & 3.25 & 3.63 & 3.95 & 4.25 & 4.35 & 2.645 & 2.420 & 2.105 & 2.005 & 1.880 & 1.555 \\
\hline $3 / 26 / 93$ & $03 p$ & 4.10 & 4.20 & 4.20 & 4.40 & 4.40 & 4.40 & 3.955 & 3.790 & 3.430 & 3.415 & 3.440 & 3.170 \\
\hline $3 / 26 / 93$ & $03 q$ & 6.80 & 11.45 & 14.25 & 19.00 & 19.00 & 22.00 & 0.325 & 0.280 & 0.230 & 0.225 & 0.225 & 0.205 \\
\hline $3 / 26 / 93$ & $03 r$ & 3.65 & 5.15 & 7.20 & 9.50 & 12.10 & 14.20 & 0.990 & 0.790 & 0.630 & 0.595 & 0.587 & 0.523 \\
\hline $3 / 26 / 93$ & $03 \mathrm{~s}$ & 3.55 & 3.60 & 3.60 & 3.80 & 4.30 & 4.85 & 2.255 & 2.075 & 1.755 & 1.630 & 1.640 & 1.285 \\
\hline $3 / 29 / 93$ & $03 t$ & 4.60 & 5.20 & 7.25 & 7.25 & 7.80 & 8.40 & 0.420 & 0.365 & 0.305 & 0.295 & 0.300 & 0.260 \\
\hline $3 / 29 / 93$ & $03 u$ & 2.50 & 2.30 & 2.30 & 2.30 & 2.30 & 2.30 & 2.165 & 2.135 & 2.095 & 2.560 & 1.825 & 2.210 \\
\hline $3 / 29 / 93$ & $03 \mathrm{v}$ & 3.30 & 3.00 & 3.00 & 3.00 & 3.00 & 3.00 & 2.715 & 2.670 & 2.710 & 2.360 & 2.730 & 3.150 \\
\hline
\end{tabular}


Data Summary

\begin{tabular}{|c|c|c|c|c|c|c|c|c|c|c|c|c|c|}
\hline DATE & EXP & $\mathrm{AH} 2$ & $\mathrm{AH} 3$ & $\mathrm{AH} 4$ & $\mathrm{AH} 5$ & $\mathrm{AH} 6$ & $\mathrm{AH7}$ & S2W & S3W & S4W & S5W & S6W & S7W \\
\hline $3 / 29 / 93$ & $03 w$ & 1.40 & 1.50 & 1.50 & 1.50 & 1.50 & 1.50 & 0.000 & 0.000 & 0.000 & 0.000 & 0.000 & 0.000 \\
\hline $3 / 29 / 93$ & $03 x$ & 2.15 & 2.15 & 2.15 & 2.15 & 2.15 & 2.15 & 1.785 & 1.860 & 1.675 & 2.180 & 1.780 & 1.505 \\
\hline $3 / 29 / 93$ & $03 y$ & 2.45 & 2.50 & 2.50 & 2.50 & 2.50 & 2.50 & 2.485 & 2.105 & 1.960 & 2.510 & 2.535 & 1.915 \\
\hline $3 / 29 / 93$ & 032 & 3.00 & 3.00 & 3.00 & 3.00 & 3.00 & 3.00 & 3.150 & 2.555 & 2.545 & 2.545 & 2.415 & 2.600 \\
\hline $3 / 29 / 93$ & $04 a$ & 4.20 & 4.20 & 4.20 & 4.20 & 4.20 & 4.20 & 4.780 & 4.070 & 3.420 & 3.675 & 4.095 & 3.517 \\
\hline $3 / 30 / 93$ & $04 \mathrm{~b}$ & 2.20 & 2.10 & 2.30 & 2.50 & 2.50 & 2.50 & 2.315 & 2.430 & 2.410 & 2.495 & 2.420 & 2.030 \\
\hline $3 / 30 / 93$ & $04 c$ & 2.50 & 2.60 & 2.60 & 2.70 & 2.70 & 2.70 & 2.840 & 2.860 & 2.695 & 2.825 & 2.660 & 2.590 \\
\hline $3 / 30 / 93$ & 04d & 3.30 & 2.90 & 2.90 & 3.10 & 3.10 & 3.10 & 3.570 & 3.160 & 3.030 & 3.060 & 3.375 & 3.060 \\
\hline $3 / 30 / 93$ & $04 e$ & 5.30 & 4.30 & 4.20 & 4.20 & 4.10 & 4.00 & 5.910 & 4.995 & 4.385 & 4.260 & 4.560 & 4.015 \\
\hline $3 / 30 / 93$ & $04 f$ & 2.50 & 2.10 & 2.40 & 2.50 & 2.50 & 2.50 & 2.525 & 2.510 & 2.405 & 2.505 & 2.705 & 2.390 \\
\hline $3 / 30 / 93$ & 049 & 2.80 & 2.70 & 2.70 & 2.90 & 2.90 & 2.90 & 3.050 & 2.995 & 2.790 & 2.950 & 3.255 & 2.800 \\
\hline $3 / 30 / 93$ & $04 h$ & 3.20 & 3.30 & 3.30 & 3.30 & 3.30 & 3.30 & 3.480 & 3.605 & 3.460 & 3.385 & 3.685 & 3.595 \\
\hline $3 / 30 / 93$ & $04 i$ & 5.50 & 4.60 & 4.10 & 4.20 & 4.30 & 4.30 & 6.970 & 5.420 & 4.515 & 4.480 & 4.915 & 4.675 \\
\hline $3 / 30 / 93$ & $04 j$ & 6.00 & 4.30 & 4.00 & 4.00 & 4.00 & 4.00 & 6.220 & 4.790 & 4.375 & 4.595 & 5.120 & 4.600 \\
\hline $4 / 1 / 93$ & $04 n$ & 3.00 & 3.00 & 3.00 & 3.00 & 3.00 & 3.00 & 3.290 & 2.940 & 2.605 & 3.020 & 3.345 & 2.870 \\
\hline $4 / 1 / 93$ & $04 k$ & 3.10 & 3.20 & 3.30 & 3.50 & 3.50 & 3.50 & 2.815 & 2.925 & 2.555 & 2.750 & 2.907 & 2.643 \\
\hline 4/1/93 & 041 & 4.10 & 4.10 & 4.20 & 4.40 & 4.60 & 4.60 & 3.225 & 3.925 & 3.475 & 3.735 & 4.130 & 3.770 \\
\hline 4/1/93 & $04 \mathrm{~m}$ & 3.50 & 3.50 & 3.50 & 3.50 & 3.50 & 3.50 & 3.630 & 2.683 & 2.485 & 2.615 & 2.765 & 2.585 \\
\hline 4/1/93 & 040 & 4.70 & 4.00 & 3.80 & 3.80 & 3.80 & 3.90 & 5.520 & 4.625 & 3.785 & 3.810 & 3.805 & 3.775 \\
\hline $4 / 1 / 93$ & $04 p$ & 3.20 & 3.20 & 3.20 & 3.20 & 3.20 & 3.20 & 2.940 & 3.005 & 2.885 & 3.185 & 3.470 & 3.305 \\
\hline $4 / 1 / 93$ & 049 & 4.50 & 3.30 & 3.30 & 3.30 & 3.30 & 3.30 & 4.910 & 4.330 & 4.190 & 4.217 & 4.285 & 4.165 \\
\hline $4 / 2 / 93$ & $04 r$ & 4.00 & 4.35 & 4.75 & 5.50 & 6.75 & 7.75 & 2.620 & 2.260 & 1.815 & 1.665 & 1.630 & 1.345 \\
\hline $4 / 5 / 93$ & $04 \mathrm{~s}$ & 0.00 & 0.00 & 0.00 & 0.00 & 0.00 & 0.00 & 1.320 & 0.900 & 0.720 & 0.000 & 0.000 & $\begin{array}{l}0.590 \\
\end{array}$ \\
\hline $4 / 5 / 93$ & $04 t$ & 0.00 & 0.00 & 0.00 & 0.00 & 0.00 & 0.00 & 0.765 & 0.570 & 0.465 & 0.440 & 0.445 & 0.390 \\
\hline $4 / 5 / 93$ & $04 u$ & 0.00 & 0.00 & 0.00 & 0.00 & 0.00 & 0.00 & 1.875 & 1.530 & 0.000 & 1.090 & 0.000 & 0.905 \\
\hline $4 / 5 / 93$ & $04 \mathrm{v}$ & 0.00 & 0.00 & 0.00 & 15.00 & 0.00 & 25.00 & 0.345 & 0.000 & 0.000 & 0.000 & 0.000 & 0.145 \\
\hline $4 / 5 / 93$ & $04 w$ & 0.00 & 0.00 & 3.90 & 0.00 & 0.00 & 6.75 & 1.160 & 0.995 & 0.780 & 0.705 & 0.690 & 0.575 \\
\hline $4 / 5 / 93$ & $04 x$ & 0.00 & 0.00 & 0.00 & 0.00 & 0.00 & 0.00 & 0.000 & 0.000 & 0.000 & 0.000 & 0.000 & 0.000 \\
\hline $4 / 5 / 93$ & $04 y$ & 2.00 & 0.00 & 2.00 & 0.00 & 2.10 & 2.10 & 2.060 & 2.225 & 2.150 & 2.130 & 2.355 & 2.130 \\
\hline $4 / 5 / 93$ & 042 & 2.50 & 0.00 & 2.60 & 2.70 & 2.70 & 2.80 & 2.615 & 2.605 & 2.395 & 2.375 & 2.585 & 2.350 \\
\hline $4 / 5 / 93$ & $05 a$ & 2.50 & 2.40 & 2.40 & 2.40 & 2.40 & 2.40 & 2.325 & 2.395 & 2.285 & 2.415 & 2.630 & 2.295 \\
\hline $4 / 5 / 93$ & $05 b$ & 0.00 & 0.00 & 0.00 & 0.00 & 0.00 & 0.00 & 3.510 & 2.990 & 2.435 & 2.700 & 3.255 & 3.005 \\
\hline $4 / 7 / 93$ & $05 c$ & 4.10 & 0.00 & 0.00 & 0.00 & 4.10 & 0.00 & 3.655 & 3.687 & 3.435 & 3.375 & 3.340 & 2.985 \\
\hline $4 / 7 / 93$ & $05 d$ & 6.90 & 0.00 & 10.00 & 0.00 & 14.00 & 22.50 & 0.310 & 0.250 & 0.200 & 0.200 & 0.200 & 0.215 \\
\hline $4 / 7 / 93$ & $05 \mathrm{e}$ & 3.80 & 4.30 & 5.90 & 6.50 & 9.25 & 10.75 & 0.880 & 0.735 & 0.623 & 0.585 & 0.595 & 0.520 \\
\hline $4 / 7 / 93$ & $05 f$ & 3.40 & 0.00 & 3.30 & 0.00 & 4.75 & 5.25 & 2.290 & 2.105 & 1.770 & 1.625 & 1.565 & 1.310 \\
\hline $4 / 12 / 93$ & $05 g$ & 3.15 & 2.80 & 3.00 & 3.00 & 3.00 & 3.00 & 3.355 & 3.030 & 2.805 & 2.895 & 3.115 & 2.900 \\
\hline $4 / 12 / 93$ & $05 \mathrm{~h}$ & 4.90 & 4.10 & 4.00 & 4.00 & 4.00 & 4.00 & 0.000 & 0.000 & 0.000 & 0.000 & 0.000 & 0.000 \\
\hline $6 / 14 / 93$ & $05 n$ & 5.25 & 4.45 & 3.90 & 3.90 & 3.95 & 4.05 & 5.620 & 4.570 & 3.770 & 3.700 & 3.980 & 3.585 \\
\hline $6 / 14 / 93$ & 050 & 3.20 & 3.80 & 3.80 & 2.90 & 2.90 & 3.15 & 3.505 & 2.925 & 2.610 & 2.950 & 3.315 & 3.020 \\
\hline $6 / 14 / 93$ & $05 p$ & 2.70 & 2.90 & 2.80 & 2.80 & 2.90 & 3.10 & 3.265 & 2.640 & 2.490 & 2.570 & 2.975 & 2.580 \\
\hline $6 / 14 / 93$ & $05 q$ & 0.00 & 0.00 & 0.00 & 0.00 & 0.00 & 0.00 & 3.340 & 2.673 & 2.420 & 2.605 & 2.710 & 2.525 \\
\hline $6 / 14 / 93$ & $05 r$ & 0.00 & 0.00 & 0.00 & 0.00 & 0.00 & 0.00 & 0.000 & 0.000 & 0.000 & 0.000 & 0.000 & 0.000 \\
\hline $6 / 14 / 93$ & $05 \mathrm{~s}$ & 0.00 & 0.00 & 0.00 & 0.00 & 0.00 & 0.00 & 0.000 & 0.000 & 0.000 & 0.000 & 0.000 & 0.000 \\
\hline $6 / 15 / 93$ & $05 t$ & 0.00 & 0.00 & 0.00 & 0.00 & 0.00 & 0.00 & 0.000 & 0.000 & 0.000 & 0.000 & 0.000 & 0.000 \\
\hline $6 / 15 / 93$ & $05 u$ & 0.00 & 0.00 & 0.00 & 0.00 & 0.00 & 0.00 & 0.000 & 0.000 & 0.000 & 0.000 & 0.000 & 0.000 \\
\hline $6 / 16 / 93$ & $05 v$ & 0.00 & 0.00 & 0.00 & 0.00 & 0.00 & 0.00 & 0.000 & 0.000 & 0.000 & 0.000 & 0.000 & 0.000 \\
\hline $6 / 17 / 93$ & $05 w$ & 0.00 & 0.00 & 0.00 & 0.00 & 0.00 & 0.00 & 0.000 & 0.000 & 0.000 & 0.000 & 0.000 & 0.000 \\
\hline $6 / 17 / 93$ & $05 x$ & 0.00 & 0.00 & 0.00 & 0.00 & 0.00 & 0.00 & 0.000 & 0.000 & 0.000 & 0.000 & 0.000 & 0.000 \\
\hline $6 / 17 / 93$ & $05 y$ & 0.00 & 0.00 & 0.00 & 0.00 & 0.00 & 0.00 & 0.000 & 0.000 & 0.000 & 0.000 & 0.000 & 0.000 \\
\hline
\end{tabular}




\begin{tabular}{|c|c|c|c|c|c|c|c|c|c|c|c|c|c|}
\hline DATE & EXP & $\mathrm{AH} 2$ & $\mathrm{AH} 3$ & $\mathrm{AH} 4$ & $\mathrm{AH} 5$ & $\mathrm{AH} 6$ & $\mathrm{AH}$ & S2W & S3W & S4W & S5W & S6W & S7W \\
\hline $6 / 17 / 93$ & $05 z$ & 0.00 & 0.00 & 0.00 & 0.00 & 0.00 & 0.00 & 0.000 & 0.000 & 0.000 & 0.000 & 0.000 & 0.000 \\
\hline $6 / 28 / 93$ & $06 a$ & 0.00 & 0.00 & 0.00 & 0.00 & 0.00 & 0.00 & 0.000 & 0.000 & 0.000 & 0.000 & 0.000 & 0.000 \\
\hline $7 / 1 / 93$ & $06 \mathrm{~b}$ & 0.00 & 0.00 & 0.00 & 4.25 & 4.25 & 4.25 & 0.000 & 0.000 & 0.000 & 0.000 & 0.000 & 0.000 \\
\hline $7 / 1 / 93$ & $06 \mathrm{c}$ & 0.00 & 0.00 & 0.00 & 4.35 & 4.35 & 4.35 & 0.000 & 0.000 & 0.000 & 0.000 & 0.000 & 0.000 \\
\hline $7 / 1 / 93$ & $06 d$ & 0.00 & 0.00 & 0.00 & 4.15 & 4.15 & 4.15 & 0.000 & 0.000 & 0.000 & 0.000 & 0.000 & 0.000 \\
\hline $7 / 1 / 93$ & $06 e$ & 0.00 & 0.00 & 0.00 & 4.30 & 4.30 & 4.30 & 0.000 & 0.000 & 0.000 & 0.000 & 0.000 & 0.000 \\
\hline $7 / 6 / 93$ & $06 f$ & 0.00 & 0.00 & 2.35 & 2.35 & 2.35 & 2.35 & 0.000 & 0.000 & 0.000 & 0.000 & 0.000 & 0.000 \\
\hline $7 / 6 / 93$ & $06 \mathrm{~h}$ & 0.00 & 0.00 & 0.00 & 3.40 & 3.40 & 3.40 & 0.000 & 0.000 & 0.000 & 0.000 & 0.000 & 0.000 \\
\hline $7 / 8 / 93$ & $06 \mathrm{~g}$ & 0.00 & 0.00 & 0.00 & 2.30 & 2.35 & 2.35 & 0.000 & 0.000 & 0.000 & 0.000 & 0.000 & 0.000 \\
\hline $7 / 8 / 93$ & $06 \mathrm{i}$ & 0.00 & 0.00 & 0.00 & 3.00 & 3.00 & 3.00 & 0.000 & 0.000 & 0.000 & 0.000 & 0.000 & 0.000 \\
\hline $7 / 8 / 93$ & $06 \mathrm{j}$ & 0.00 & 0.00 & 0.00 & 4.20 & 4.20 & 4.20 & 0.000 & 0.000 & 0.000 & 0.000 & 0.000 & 0.000 \\
\hline $7 / 8 / 93$ & 06k & 0.00 & 0.00 & 0.00 & 3.80 & 3.80 & 3.80 & 0.000 & 0.000 & 0.000 & 0.000 & 0.000 & 0.000 \\
\hline $7 / 8 / 93$ & 061 & 0.00 & 0.00 & 0.00 & 4.30 & 4.30 & 4.30 & 0.000 & 0.000 & 0.000 & 0.000 & 0.000 & 0.000 \\
\hline $7 / 8 / 93$ & $06 \mathrm{~m}$ & 0.00 & 0.00 & 0.00 & 3.70 & 3.70 & 3.70 & 0.000 & 0.000 & 0.000 & 0.000 & 0.000 & 0.000 \\
\hline $7 / 8 / 93$ & $06 n$ & 0.00 & 0.00 & 0.00 & 4.30 & 4.30 & 4.30 & 0.000 & 0.000 & 0.000 & 0.000 & 0.000 & 0.000 \\
\hline $7 / 8 / 93$ & 060 & 0.00 & 0.00 & 0.00 & 3.20 & 3.20 & 3.20 & 0.000 & 0.000 & 0.000 & 0.000 & 0.000 & 0.000 \\
\hline $7 / 12 / 93$ & $06 p$ & 0.00 & 0.00 & 0.00 & 4.00 & 4.00 & 4.00 & 0.000 & 0.000 & 0.000 & 0.000 & 0.000 & 0.000 \\
\hline $7 / 12 / 93$ & $06 q$ & 0.00 & 0.00 & 0.00 & 3.00 & 3.00 & 3.00 & 0.000 & 0.000 & 0.000 & 0.000 & 0.000 & 0.000 \\
\hline $7 / 12 / 93$ & $06 r$ & 0.00 & 0.00 & 0.00 & 4.00 & 4.00 & 4.00 & 0.000 & 0.000 & 0.000 & 0.000 & 0.000 & 0.000 \\
\hline $7 / 12 / 93$ & $06 s$ & 0.00 & 0.00 & 0.00 & 3.00 & 3.00 & 3.00 & 0.000 & 0.000 & 0.000 & 0.000 & 0.000 & 0.000 \\
\hline $7 / 12 / 93$ & $06 t$ & 0.00 & 0.00 & 0.00 & 4.00 & 4.00 & 4.00 & 0.000 & 0.000 & 0.000 & 0.000 & 0.000 & 0.000 \\
\hline $7 / 12 / 93$ & $06 u$ & 0.00 & 0.00 & 0.00 & 3.00 & 3.00 & 3.00 & 0.000 & 0.000 & 0.000 & 0.000 & 0.000 & 0.000 \\
\hline $7 / 12 / 93$ & $06 \mathrm{v}$ & 0.00 & 0.00 & 0.00 & 3.90 & 3.90 & 3.90 & 0.000 & 0.000 & 0.000 & 0.000 & 0.000 & 0.000 \\
\hline $7 / 13 / 93$ & $06 w$ & 0.00 & 0.00 & 0.00 & 3.10 & 3.10 & 3.10 & 0.000 & 0.000 & 0.000 & 0.000 & 0.000 & 0.000 \\
\hline $7 / 13 / 93$ & $06 x$ & 0.00 & 0.00 & 0.00 & 3.85 & 3.85 & 3.85 & 0.000 & 0.000 & 0.000 & 0.000 & 0.000 & 0.000 \\
\hline $7 / 13 / 93$ & $06 y$ & 0.00 & 0.00 & 0.00 & 3.25 & 3.25 & 3.20 & 0.000 & 0.000 & 0.000 & 0.000 & 0.000 & 0.000 \\
\hline $7 / 13 / 93$ & $06 z$ & 0.00 & 0.00 & 0.00 & 3.70 & 3.70 & 3.70 & 0.000 & 0.000 & 0.000 & 0.000 & 0.000 & 0.000 \\
\hline $7 / 17 / 93$ & $07 a$ & 0.00 & 0.00 & 0.00 & 3.20 & 3.20 & 3.20 & 0.000 & 0.000 & 0.000 & 0.000 & 0.000 & 0.000 \\
\hline $7 / 17 / 93$ & $07 \mathrm{~b}$ & 0.00 & 0.00 & 0.00 & 3.70 & 3.70 & 3.70 & 0.000 & 0.000 & 0.000 & 0.000 & 0.000 & 0.000 \\
\hline $7 / 17 / 93$ & $07 c$ & 0.00 & 0.00 & 0.00 & 2.90 & 2.90 & 2.90 & 0.000 & 0.000 & 0.000 & 0.000 & 0.000 & 0.000 \\
\hline $7 / 19 / 93$ & 07d & 0.00 & 0.00 & 0.00 & 3.70 & 3.70 & 3.70 & 0.000 & 0.000 & 0.000 & 0.000 & 0.000 & 0.000 \\
\hline $7 / 19 / 93$ & $07 e$ & 0.00 & 0.00 & 0.00 & 0.00 & 0.00 & 0.00 & 0.000 & 0.000 & 0.000 & 0.000 & 0.000 & 0.000 \\
\hline $7 / 19 / 93$ & $07 f$ & 0.00 & 0.00 & 0.00 & 0.00 & 0.00 & 0.00 & 0.000 & 0.000 & 0.000 & 0.000 & 0.000 & 0.000 \\
\hline $7 / 19 / 93$ & $07 \mathrm{~g}$ & 0.00 & 0.00 & 0.00 & 3.10 & 3.10 & 3.10 & 0.000 & 0.000 & 0.000 & 0.000 & 0.000 & 0.000 \\
\hline $7 / 19 / 93$ & $07 \mathrm{~h}$ & 0.00 & 0.00 & 0.00 & 3.90 & 3.90 & 3.90 & 0.000 & 0.000 & 0.000 & 0.000 & 0.000 & 0.000 \\
\hline $7 / 20 / 93$ & $07 i$ & 0.00 & 0.00 & 0.00 & 3.40 & 3.40 & 3.40 & 0.000 & 0.000 & 0.000 & 0.000 & 0.000 & 0.000 \\
\hline $7 / 20 / 93$ & 07j & 0.00 & 0.00 & 0.00 & 3.80 & 3.80 & 3.80 & 0.000 & 0.000 & 0.000 & 0.000 & 0.000 & 0.000 \\
\hline $7 / 20 / 93$ & $07 k$ & 0.00 & 0.00 & 0.00 & 3.00 & 3.00 & 3.00 & 0.000 & 0.000 & 0.000 & 0.000 & 0.000 & 0.000 \\
\hline $7 / 20 / 93$ & 071 & 0.00 & 0.00 & 0.00 & 4.20 & 4.00 & 4.20 & 0.000 & 0.000 & 0.000 & 0.000 & 0.000 & 0.000 \\
\hline $7 / 20 / 93$ & $07 \mathrm{~m}$ & 0.00 & 0.00 & 0.00 & 3.00 & 3.00 & 3.00 & 0.000 & 0.000 & 0.000 & 0.000 & 0.000 & 0.000 \\
\hline $7 / 20 / 93$ & $07 n$ & 0.00 & 0.00 & 0.00 & 3.80 & 3.80 & 3.80 & 0.000 & 0.000 & 0.000 & 0.000 & 0.000 & 0.000 \\
\hline $7 / 28 / 93$ & 070 & 0.00 & 0.00 & 0.00 & 4.30 & 4.30 & 4.30 & 0.000 & 0.000 & 0.000 & 0.000 & 0.000 & 0.000 \\
\hline $7 / 28 / 93$ & $07 p$ & 0.00 & 0.00 & 0.00 & 4.20 & 4.20 & 4.20 & 0.000 & 0.000 & 0.000 & 0.000 & 0.000 & 0.000 \\
\hline $7 / 28 / 93$ & $07 q$ & 0.00 & 0.00 & 0.00 & 4.40 & 4.40 & 4.40 & 0.000 & 0.000 & 0.000 & 0.000 & 0.000 & 0.000 \\
\hline $7 / 29 / 93$ & $07 r$ & 0.00 & 0.00 & 0.00 & 2.70 & 2.70 & 2.70 & 0.000 & 0.000 & 0.000 & 0.000 & 0.000 & 0.000 \\
\hline $7 / 29 / 93$ & $07 s$ & 0.00 & 0.00 & 0.00 & 4.10 & 4.10 & 4.10 & 0.000 & 0.000 & 0.000 & 0.000 & 0.000 & 0.000 \\
\hline $7 / 29 / 93$ & $07 t$ & 0.00 & 0.00 & 0.00 & 3.60 & 3.60 & 3.60 & 0.000 & 0.000 & 0.000 & 0.000 & 0.000 & 0.000 \\
\hline $8 / 4 / 93$ & $07 u$ & 0.00 & 0.00 & 0.00 & 2.80 & 2.80 & 2.80 & 0.000 & 0.000 & 0.000 & 0.000 & 0.000 & 0.000 \\
\hline $8 / 4 / 93$ & $07 v$ & 0.00 & 0.00 & 0.00 & 3.95 & 3.85 & 3.85 & 0.000 & 0.000 & 0.000 & 0.000 & 0.000 & 0.000 \\
\hline $8 / 5 / 93$ & $07 w$ & 0.00 & 0.00 & 0.00 & 3.90 & 3.90 & 3.90 & 0.000 & 0.000 & 0.000 & 0.000 & 0.000 & 0.000 \\
\hline
\end{tabular}


Data Summary

\begin{tabular}{|c|c|c|c|c|c|c|c|c|c|c|c|c|c|}
\hline DATE & EXP & $\mathrm{AH} 2$ & $\mathrm{AH3}$ & $\mathrm{AH} 4$ & $\mathrm{AH5}$ & $\mathrm{AH} 6$ & AH7 & S2W & S3W & S4W & S5W & S6W & S7W \\
\hline $8 / 5 / 93$ & $07 x$ & 0.00 & 0.00 & 0.00 & 4.20 & 4.20 & 4.20 & 0.000 & 0.000 & 0.000 & 0.000 & 0.000 & 0.000 \\
\hline $8 / 5 / 93$ & $07 y$ & 0.00 & 0.00 & 0.00 & 3.90 & 3.90 & 3.90 & 0.000 & 0.000 & 0.000 & 0.000 & 0.000 & 0.000 \\
\hline $8 / 12 / 93$ & $07 z$ & 0.00 & 0.00 & 0.00 & 4.20 & 4.30 & 4.30 & 0.000 & 0.000 & 0.000 & 0.000 & 0.000 & 0.000 \\
\hline $8 / 12 / 93$ & $08 a$ & 0.00 & 0.00 & 0.00 & 2.80 & 2.80 & 2.80 & 0.000 & 0.000 & 0.000 & 0.000 & 0.000 & 0.000 \\
\hline $8 / 12 / 93$ & $08 b$ & 0.00 & 0.00 & 0.00 & 3.70 & 3.70 & 3.70 & 0.000 & 0.000 & 0.000 & 0.000 & 0.000 & 0.000 \\
\hline $8 / 17 / 93$ & $08 \mathrm{C}$ & 3.40 & 3.05 & 2.95 & 3.40 & 3.15 & 3.00 & 3.635 & 3.180 & 2.920 & 3.270 & 3.420 & 2.945 \\
\hline $8 / 17 / 93$ & $08 \mathrm{~d}$ & 0.00 & 0.00 & 0.00 & 0.00 & 0.00 & 0.00 & 0.000 & 0.000 & 0.000 & 0.000 & 0.000 & 0.000 \\
\hline $8 / 17 / 93$ & $08 \mathrm{e}$ & 00 & 0.00 & 0.00 & 0.00 & 0.00 & 0.00 & 0.000 & 0.000 & 0.000 & 0.000 & 0.000 & 0.000 \\
\hline $8 / 17 / 93$ & $08 \mathrm{f}$ & 0.00 & 0.00 & 0.00 & 0.00 & 0.00 & 0.00 & 0.000 & 0.000 & 0.000 & 0.000 & 0.000 & 0.000 \\
\hline $8 / 17 / 93$ & $08 \mathrm{~g}$ & 0.00 & 0.00 & 0.00 & 0.00 & 0.00 & 0.00 & 0.000 & 0.000 & 0.000 & 0.000 & 0.000 & 0.000 \\
\hline $8 / 17 / 93$ & $08 \mathrm{~h}$ & 0.00 & 0.00 & 0.00 & 0.00 & 0.00 & 0.00 & 0.000 & 0.000 & 0.000 & 0.000 & 0.000 & 0.000 \\
\hline $8 / 17 / 93$ & $08 i$ & 0.00 & 0.00 & 0.00 & 0.00 & 0.00 & 0.00 & 0.000 & 0.000 & 0.000 & 0.000 & 0.000 & 0.000 \\
\hline $8 / 17 / 93$ & $08 \mathrm{j}$ & 0.00 & 0.00 & 0.00 & 0.00 & 0.00 & 0.00 & 0.000 & 0.000 & 0.000 & 0.000 & 0.000 & 0.000 \\
\hline $8 / 31 / 93$ & $08 \mathrm{k}$ & 2.90 & 2.80 & 2.80 & 2.80 & 2.80 & 2.80 & 3.170 & 2.710 & 2.540 & \begin{tabular}{|l}
2.855 \\
\end{tabular} & 3.195 & 2.835 \\
\hline $9 / 1 / 93$ & 081 & 3.35 & 2.90 & 2.95 & 3.05 & 3.05 & 3.05 & 3.607 & 2.710 & 2.805 & 2.767 & 3.015 & 3.045 \\
\hline $9 / 1 / 93$ & $08 \mathrm{~m}$ & 3.05 & 2.80 & 2.80 & 2.90 & 3.05 & 3.05 & 3.065 & 2.650 & 2.560 & 2.840 & 3.145 & 2.720 \\
\hline $9 / 1 / 93$ & $08 n$ & 3.30 & 2.90 & 2.90 & 3.05 & 3.05 & 3.05 & 3.590 & 2.840 & 2.570 & 2.870 & 3.300 & 2.810 \\
\hline $9 / 2 / 93$ & 080 & 3.20 & 2.85 & 2.85 & 2.90 & 3.00 & 3.05 & 3.335 & 2.700 & 2.505 & 2.900 & 977 & 660 \\
\hline $9 / 2 / 93$ & $08 p$ & 2.90 & 2.75 & 2.75 & 2.80 & 2.85 & 2.95 & 2.985 & 2.640 & 2.425 & 2.660 & 2.770 & 460 \\
\hline $9 / 2 / 93$ & $08 q$ & 3.10 & 2.85 & 2.95 & 3.05 & 3.05 & 2.95 & 3.560 & 2.845 & 2.460 & 2.495 & 2.790 & 2.525 \\
\hline $9 / 2 / 93$ & $08 r$ & 2.80 & 2.60 & 2.60 & 2.60 & 2.75 & 2.85 & 3.110 & 2.565 & 2.405 & 2.655 & 2.920 & 2.630 \\
\hline $9 / 6 / 93$ & 08s & 3.10 & 2.80 & 3.05 & 2.80 & 2.75 & 2.80 & 3.290 & 2.780 & 2.375 & 2.643 & 2.980 & 2.310 \\
\hline $9 / 6 / 93$ & $08 \mathrm{t}$ & 2.70 & 2.55 & 2.65 & 2.60 & 2.65 & 2.45 & 2.683 & 2.340 & 2.255 & 2.370 & 2.630 & 2.370 \\
\hline $9 / 6 / 93$ & $08 u$ & 3.10 & 2.75 & 2.85 & 2.75 & 2.70 & 2.70 & 3.000 & 2.595 & 2.535 & 2.655 & 3.085 & 2.745 \\
\hline $9 / 6 / 93$ & $08 \mathrm{~V}$ & 2.70 & 2.55 & 2.60 & 2.65 & 2.70 & 2.55 & 2.675 & 2.410 & 2.365 & 2.325 & 2.985 & 2.335 \\
\hline $9 / 6 / 93$ & $08 w$ & 2.95 & 2.80 & 2.85 & 2.75 & 2.80 & 2.80 & 3.050 & 2.650 & 2.450 & 2.880 & 2.600 & 2.490 \\
\hline $9 / 6 / 93$ & $08 x$ & 2.85 & 2.70 & 2.70 & 2.70 & 2.70 & 2.70 & 2.655 & 2.550 & 2.275 & 2.460 & 2.375 & 2.200 \\
\hline $9 / 7 / 93$ & $08 y$ & 2.90 & 2.95 & 2.80 & 2.90 & 2.80 & 3.15 & 3.125 & 2.600 & 2.380 & 2.640 & 2.970 & 2.685 \\
\hline $9 / 7 / 93$ & $08 z$ & 2.75 & 2.65 & 2.70 & 2.75 & 2.75 & 2.75 & 2.565 & 2.380 & 2.155 & 2.695 & 2.850 & 2.235 \\
\hline $9 / 7 / 93$ & $09 a$ & 2.85 & 2.95 & 3.00 & 3.00 & 3.20 & 3.20 & 2.915 & 2.535 & 2.510 & 2.525 & 2.885 & 2.640 \\
\hline $9 / 7 / 93$ & $09 b$ & 2.70 & 2.85 & 2.75 & 2.70 & 2.70 & 2.65 & 2.530 & 2.465 & 2.215 & 2.305 & 2.610 & 2.265 \\
\hline $9 / 7 / 93$ & $09 \mathrm{C}$ & 3.15 & 2.90 & 3.00 & 3.05 & 3.05 & 2.90 & 3.540 & 2.975 & 2.660 & 2.960 & 3.300 & 2.915 \\
\hline $9 / 7 / 93$ & 09d & 2.85 & 2.75 & 2.70 & 2.95 & 2.85 & 2.80 & 2.975 & 2.730 & 2.415 & 2.800 & 3.090 & 2.500 \\
\hline $9 / 8 / 93$ & $09 e$ & 3.15 & 2.90 & 2.85 & 2.90 & 2.95 & 2.90 & 3.380 & 2.645 & 2.705 & 3.000 & 3.200 & 2.680 \\
\hline $9 / 8 / 93$ & $09 f$ & 2.80 & 2.70 & 2.65 & 2.80 & 2.85 & 2.65 & 3.010 & 2.955 & 2.895 & 2.745 & 3.100 & 2.695 \\
\hline $9 / 13 / 93$ & $09 g$ & 2.95 & 3.00 & 3.10 & 3.35 & 3.40 & 3.25 & 2.960 & 2.635 & 2.665 & 2.695 & 2.945 & 2.700 \\
\hline 9/13/93 & 099 & 2.95 & 3.00 & 3.10 & 3.35 & 3.40 & 3.25 & 2.960 & 2.635 & 2.665 & \begin{tabular}{|l|}
2.695 \\
\end{tabular} & 2.945 & 2.700 \\
\hline $9 / 13 / 93$ & $09 \mathrm{~h}$ & 2.75 & 3.00 & 2.85 & 3.25 & 3.30 & 2.75 & 2.450 & 2.455 & 2.100 & 2.725 & 2.905 & 2.210 \\
\hline 9/14/93 & $09 i$ & 3.00 & 3.10 & 3.10 & 3.10 & 3.10 & 3.10 & 2.715 & 2.845 & 2.600 & 2.545 & 2.730 & 2.585 \\
\hline $9 / 14 / 93$ & $09 i$ & 3.00 & 3.20 & 3.20 & 3.25 & 3.25 & 3.30 & 2.740 & 2.690 & 2.500 & 2.565 & 2.795 & 2.655 \\
\hline $9 / 14 / 93$ & $09 j$ & 2.80 & 3.00 & 2.90 & 2.95 & 2.80 & 2.90 & 2.360 & 2.415 & 2.380 & 2.385 & 2.560 & 2.390 \\
\hline 9/14/93 & $09 k$ & 3.00 & 3.00 & 3.15 & 3.10 & 3.05 & 3.05 & 2.770 & 2.615 & 2.460 & 2.535 & 2.715 & 2.585 \\
\hline $9 / 14 / 93$ & 091 & 2.90 & 2.80 & 2.90 & 2.95 & 2.90 & 3.05 & 2.345 & 2.485 & 2.285 & 2.290 & 2.415 & 2.260 \\
\hline $9 / 14 / 93$ & $09 m$ & 2.95 & 3.05 & 3.00 & 3.05 & 3.00 & 3.05 & 2.620 & 2.595 & 2.490 & 2.490 & 2.525 & 2.320 \\
\hline $9 / 15 / 93$ & $09 n$ & 3.00 & 3.10 & 3.10 & 3.20 & 3.30 & 3.30 & 2.448 & 2.338 & 2.108 & 2.140 & 2.193 & 2.015 \\
\hline $9 / 17 / 93$ & 090 & 3.10 & 3.30 & 3.40 & 3.40 & 3.40 & 3.40 & 2.755 & 2.520 & 2.385 & 2.495 & 2.580 & 2.350 \\
\hline $9 / 17 / 93$ & $09 p$ & 3.10 & 3.30 & 3.40 & 3.40 & 3.40 & 3.40 & 2.670 & 2.700 & 2.445 & 2.490 & 2.605 & 2.310 \\
\hline $9 / 17 / 93$ & $09 q$ & 3.10 & 3.30 & 3.40 & 3.40 & 3.40 & 3.40 & 2.635 & 2.600 & 2.470 & 2.475 & 2.640 & 2.375 \\
\hline $9 / 20 / 93$ & $09 r$ & 3.00 & 3.15 & 3.20 & 3.05 & 3.10 & 3.35 & 2.760 & 2.570 & 2.420 & 2.455 & 2.765 & 2.415 \\
\hline $9 / 20 / 93$ & $09 \mathrm{~s}$ & 3.10 & 3.10 & 0.00 & 0.00 & 0.00 & 0.00 & 2.645 & 2.665 & 2.430 & 2.475 & 2.810 & 2.420 \\
\hline
\end{tabular}




\begin{tabular}{|c|c|c|c|c|c|c|c|c|c|c|c|c|c|}
\hline & EXP & $\mathrm{AH} 2$ & $\mathrm{AH} 3$ & $\mathrm{AH} 4$ & $\mathrm{H} 5$ & $\mathrm{H} 6$ & תוז & S2W & S3W & S4W & S5W & S6W & S7W \\
\hline $9 / 20 / 93$ & $09 t$ & 3.10 & 3.20 & & .30 & .20 & 3.15 & 2.645 & 2.590 & 2.450 & 2.525 & 2.625 & \\
\hline & $9 u$ & & & & 10 & .00 & 3.05 & .549 & 2.715 & 2.450 & 0.000 & 2.760 & 2.400 \\
\hline $9 / 21 / 93$ & $9 v$ & & 10 & 15 & 05 & 3.15 & 3.25 & 2.765 & 2.665 & 2.330 & .355 & .625 & 2.285 \\
\hline $21 / 93$ & $9 w$ & 15 & 25 & 15 & .25 & 3.20 & 3.10 & 2.635 & 2.580 & 2.365 & 2.480 & 8.875 & 2.375 \\
\hline $21 / 93$ & & .05 & & & .15 & 3.05 & 3.30 & 2.680 & 2.725 & 2.310 & 2.400 & 2.955 & 2.340 \\
\hline $22 / 93$ & & & & & 30 & & 3.25 & & 0.000 & & 0.000 & 0.000 & 0.000 \\
\hline & & & & & & & 0.00 & & & & 0.000 & 0.000 & .000 \\
\hline & & & & & 00 & .00 & 0.00 & & & & & & \\
\hline & & & & & & .00 & 0.00 & 0.000 & 0.000 & & 0.000 & 0.000 & .000 \\
\hline & & & & & .00 & .00 & 0.00 & 0.000 & 0.000 & 0.000 & 0.000 & 0.000 & 0.000 \\
\hline & & & & & & & 0.00 & 0.000 & 0.000 & & 0.000 & 0.000 & 0.000 \\
\hline & & & & & .00 & 0.00 & 0.00 & 0.000 & 0.000 & 0.000 & 0.000 & 0.000 & .000 \\
\hline $9 / 23 / 93$ & & .00 & & & .00 & 0.00 & 0.00 & 0.000 & 0.000 & 0.000 & 0.000 & 0.000 & .000 \\
\hline & & & & & .00 & 0.00 & 0.00 & 0.000 & 0.000 & 0.000 & 0.000 & 0.000 & .000 \\
\hline & & & & & .00 & 0.00 & 0.00 & 0.000 & 0.000 & 0.000 & 0.000 & 0.000 & .000 \\
\hline & & & & & & & 0.00 & & & & 0.000 & & \\
\hline & & & & & & & & & & & 0.000 & & \\
\hline & & & & & & & & & & & & & \\
\hline & & & & & & .0 & & & & & 0.000 & 000 & 300 \\
\hline & & & & & & & .35 & 25 & 60 & & 2.550 & .005 & 400 \\
\hline & & & & & & & & & & & 2.480 & 2.945 & 140 \\
\hline & & & & & & & .40 & 2.770 & 2.745 & & 2.460 & 2.775 & 305 \\
\hline & & & & & .45 & .45 & 3.45 & 2.760 & 2.770 & & 2.670 & 2.800 & \\
\hline & & & & & & & 3.20 & 2.670 & 2.670 & & 2.665 & 2.950 & 2.570 \\
\hline & & & & & & & 3.20 & 2.735 & & & 2.540 & 2.840 & 2.560 \\
\hline & & & & & & & 3.20 & 2.710 & 2.565 & 2.505 & 2.650 & 3.040 & 2.520 \\
\hline & & & & & & .45 & 3.35 & 2.825 & 2.565 & 2.490 & 3.120 & 3.695 & 2.735 \\
\hline & & & & & & & 3.35 & & 2.720 & & 2.615 & 3.095 & 2.625 \\
\hline & & & & & & & 3.40 & 2.3 & 2.635 & & 2.315 & 2.660 & 2.770 \\
\hline & & & & & & & & & & & 2.755 & 2.865 & \\
\hline & & & & & & & & & & & .825 & 575 & \\
\hline & & & & & & & 2.85 & 3.2 & 2.6 & 80 & 2.895 & 2.955 & 2.640 \\
\hline & & & & & & & 2.85 & 3.0 & 2.740 & 2.3 & 2.725 & 3.035 & 2.735 \\
\hline & & & & & & & 2.80 & 3.1 & 2.620 & 2.3 & 2.850 & 3.345 & 690 \\
\hline & & & & & & 2.75 & 2.85 & 3.1 & 2.735 & 2.520 & 2.585 & 2.660 & 2.555 \\
\hline & & & & & & & 2.75 & 3.070 & 2.660 & 2.495 & 2.805 & 2.795 & 2.570 \\
\hline & & & & & & & & & 2.5 & & 2.640 & 3.195 & \\
\hline & & & & & & & 2.85 & & 2.7 & 2.570 & 2.945 & 3.150 & 615 \\
\hline & & & & & & & & & 2.6 & 2.4 & 3.160 & 3.650 & \\
\hline & & & & & & & & & & & $\overline{00}$ & & $\overline{645}$ \\
\hline & & & & & & & 2.95 & & & & 2.873 & & \\
\hline & & & & & & & 2.85 & & & & 2.790 & & \\
\hline & & & & & & & & & & & 3.025 & & \\
\hline & & & & & & & 2.95 & & 2.720 & 2.585 & 3.480 & 3.640 & 2.725 \\
\hline & & & & & & & 2.95 & 3.0 & 2.575 & 2.490 & 2.975 & 3.130 & 2.665 \\
\hline & & & & & 3.0 & 2.7 & 3.05 & 10 & 2.705 & 2.580 & 2.770 & 3.035 & 755 \\
\hline & & & & & & 2.9 & 2.90 & 2.9 & 2.705 & 2.515 & 3.115 & 3.680 & 380 \\
\hline & & & & & & 3 & 3.00 & 65 & 2.555 & 2.610 & 3.360 & 3.980 & 2.815 \\
\hline & & & & & & & 3.00 & & & 2.5 & 2.750 & 2.870 & 2.620 \\
\hline $10 / 25 / 93$ & $11 \mathrm{u}$ & & & 3.00 & 2.90 & 2.90 & 2.90 & 3.065 & 2.645 & 2.490 & 2.905 & 3.120 & 2.440 \\
\hline
\end{tabular}




\begin{tabular}{|c|c|c|c|c|c|c|c|c|c|c|c|c|c|}
\hline DATE & EXP & $\mathrm{AH2}$ & $\mathrm{AH} 3$ & $\mathrm{AH} 4$ & $\mathrm{AH5}$ & $\mathrm{AH} 6$ & $\mathrm{AH7}$ & S2W & S3W & S4W & S5W & S6W & S7W \\
\hline $10 / 25 / 93$ & $11 \mathrm{v}$ & 3.00 & 2.90 & 2.90 & 2.80 & 2.80 & 2.85 & 3.180 & 2.610 & 2.615 & 2.775 & 3.065 & 2.820 \\
\hline $10 / 25 / 93$ & $11 w$ & 2.95 & 2.90 & 3.05 & 3.10 & 2.90 & 2.90 & 3.150 & 2.680 & 2.470 & 3.030 & 3.480 & 2.730 \\
\hline $10 / 25 / 93$ & $11 x$ & 3.05 & 2.95 & 2.90 & 3.05 & 3.05 & 2.90 & 2.975 & 2.580 & 2.630 & 3.975 & 3.655 & 2.910 \\
\hline $11 / 14 / 93$ & $10 x$ & 3.30 & 3.35 & 3.45 & 3.45 & 3.35 & 3.45 & 2.750 & 2.745 & 2.325 & 2.460 & 2.825 & 2.510 \\
\hline $11 / 21 / 93$ & 110 & 3.10 & 3.15 & 2.90 & 3.00 & 3.10 & 3.00 & 2.915 & 2.535 & 2.590 & 2.420 & 2.775 & 2.930 \\
\hline $2 / 23 / 94$ & & 3.35 & 3.45 & 3.60 & 3.70 & 3.90 & 3.95 & & & & & & \\
\hline $2 / 23 / 94$ & & 3.45 & 3.60 & 3.65 & 3.85 & 4.25 & 4.30 & & & & & & \\
\hline $2 / 23 / 94$ & & 3.35 & 3.55 & 3.60 & 3.75 & 4.10 & 4.30 & & & & & & \\
\hline $2 / 24 / 94$ & & 3.40 & 3.45 & 3.60 & 3.80 & 4.10 & 4.30 & & & & & & \\
\hline $4 / 18 / 94$ & $12 a$ & 0.00 & 0.00 & 0.00 & 0.00 & 0.00 & 0.00 & 0.000 & 0.000 & 0.000 & 0.000 & 0.000 & 0.000 \\
\hline $4 / 18 / 94$ & $12 b$ & 0.00 & 0.00 & 0.00 & 0.00 & 0.00 & 0.00 & 0.000 & 0.000 & 0.000 & 0.000 & 0.000 & 0.000 \\
\hline $4 / 18 / 94$ & $12 \mathrm{c}$ & 0.00 & 0.00 & 0.00 & 0.00 & 0.00 & 0.00 & 0.000 & 0.000 & 0.000 & 0.000 & 0.000 & 0.000 \\
\hline $4 / 18 / 94$ & $12 d$ & 0.00 & 0.00 & 0.00 & 0.00 & 0.00 & 0.00 & 0.000 & 0.000 & 0.000 & 0.000 & 0.000 & 0.000 \\
\hline $4 / 18 / 94$ & $12 e$ & 0.00 & 0.00 & 0.00 & 0.00 & 0.00 & 0.00 & 0.000 & 0.000 & 0.000 & 0.000 & 0.000 & 0.000 \\
\hline $4 / 18 / 94$ & $A 12$ & 0.00 & 0.00 & 0.00 & 0.00 & 0.00 & 0.00 & & & & & & \\
\hline $4 / 21 / 94$ & B12 & 0.00 & 0.00 & 0.00 & 0.00 & 0.00 & 0.00 & & & & & & \\
\hline $5 / 2 / 94$ & $12 f$ & 0.00 & 0.00 & 0.00 & 0.00 & 0.00 & 0.00 & 0.000 & 0.000 & 0.000 & 0.000 & 0.000 & 0.000 \\
\hline $5 / 2 / 94$ & $12 \mathrm{~g}$ & 0.00 & 0.00 & 0.00 & 0.00 & 0.00 & 0.00 & 0.000 & 0.000 & 0.000 & 0.000 & 0.000 & 0.000 \\
\hline $5 / 2 / 94$ & $12 h$ & 0.00 & 0.00 & 0.00 & 0.00 & 0.00 & 0.00 & 0.000 & 0.000 & 0.000 & 0.000 & 0.000 & 0.000 \\
\hline $5 / 2 / 94$ & $12 i$ & 0.00 & 0.00 & 0.00 & 0.00 & 0.00 & 0.00 & 0.000 & 0.000 & 0.000 & 0.000 & 0.000 & 0.000 \\
\hline $5 / 2 / 94$ & $12 j$ & 3.70 & 3.50 & 3.50 & 3.40 & 3.40 & 3.40 & 5.275 & 4.915 & 3.850 & 4.025 & 4.310 & 3.180 \\
\hline $5 / 3 / 94$ & $12 r$ & 3.05 & 3.10 & 3.10 & 3.20 & 3.00 & 2.60 & 3.510 & 3.700 & 3.275 & 3.175 & 3.140 & 2.440 \\
\hline $5 / 3 / 94$ & $12 k$ & 0.00 & 0.00 & 0.00 & 0.00 & 0.00 & 0.00 & 0.000 & 0.000 & 0.000 & 0.000 & 0.000 & 0.000 \\
\hline $5 / 3 / 94$ & 121 & 0.00 & 0.00 & 0.00 & 0.00 & 0.00 & 0.00 & 0.000 & 0.000 & 0.000 & 0.000 & 0.000 & 0.000 \\
\hline $5 / 3 / 94$ & $12 \mathrm{~m}$ & 0.00 & 0.00 & 0.00 & 0.00 & 0.00 & 0.00 & 0.000 & 0.000 & 0.000 & 0.000 & 0.000 & 0.000 \\
\hline $5 / 3 / 94$ & $12 n$ & 0.00 & 0.00 & 0.00 & 0.00 & 0.00 & 0.00 & 0.000 & 0.000 & 0.000 & 0.000 & 0.000 & 0.000 \\
\hline $5 / 3 / 94$ & 120 & 3.70 & 3.50 & 3.40 & 3.60 & 3.40 & 3.20 & 4.290 & 3.955 & 3.600 & 3.610 & 3.675 & 2.925 \\
\hline $5 / 3 / 94$ & $12 p$ & 0.00 & 0.00 & 0.00 & 0.00 & 0.00 & 0.00 & 0.000 & 0.000 & 0.000 & 0.000 & 0.000 & 0.000 \\
\hline $5 / 3 / 94$ & $12 q$ & 0.00 & 0.00 & 0.00 & 0.00 & 0.00 & 0.00 & 0.000 & 0.000 & 0.000 & 0.000 & 0.000 & 0.000 \\
\hline $5 / 3 / 94$ & 125 & 3.70 & 3.45 & 3.30 & 3.35 & 3.45 & 3.35 & 4.080 & 3.945 & 3.630 & 3.620 & 3.680 & 2.870 \\
\hline $5 / 3 / 94$ & $12 \mathrm{t}$ & 3.75 & 3.60 & 3.45 & 3.50 & 3.45 & 3.45 & 4.555 & 4.430 & 3.845 & 4.075 & 4.255 & 3.555 \\
\hline $5 / 5 / 94$ & $12 u$ & 0.00 & 0.00 & 0.00 & 0.00 & 0.00 & 0.00 & 0.000 & 0.000 & 0.000 & 0.000 & 0.000 & 0.000 \\
\hline $5 / 5 / 94$ & $12 v$ & 2.65 & 2.70 & 2.65 & 2.75 & 2.65 & 2.45 & 3.010 & 2.940 & 2.785 & 2.785 & 2.895 & 2.290 \\
\hline $5 / 6 / 94$ & $12 w$ & 2.60 & 2.50 & 2.40 & 2.45 & 2.40 & 2.55 & 2.710 & 2.505 & 2.295 & 2.355 & 2.593 & 2.405 \\
\hline $5 / 6 / 94$ & $12 x$ & 3.10 & 3.00 & 2.70 & 2.70 & 2.70 & 2.65 & 3.295 & 3.235 & 2.995 & \begin{tabular}{|l|}
2.995 \\
\end{tabular} & 3.170 & 2.855 \\
\hline $5 / 6 / 94$ & $12 y$ & 4.85 & 4.00 & 3.60 & 3.50 & 3.40 & 3.20 & 5.345 & 4.345 & 3.720 & 3.565 & 3.690 & 3.320 \\
\hline $5 / 6 / 94$ & $12 z$ & 2.10 & 2.25 & 2.15 & 2.25 & 2.25 & 2.25 & 2.045 & 1.995 & 2.025 & 2.070 & 1.990 & 1.800 \\
\hline $5 / 6 / 94$ & $13 a$ & 2.40 & 2.50 & 2.50 & 2.50 & 2.55 & 2.45 & 2.635 & 2.540 & 2.570 & 2.525 & 2.585 & 2.490 \\
\hline $5 / 6 / 94$ & $13 b$ & 3.15 & 2.95 & 2.85 & 2.95 & 2.85 & 2.85 & 3.240 & 2.935 & 2.825 & 2.865 & 2.965 & 2.765 \\
\hline $5 / 6 / 94$ & $13 c$ & 4.80 & 3.90 & 3.40 & 3.40 & 3.35 & 3.30 & 5.435 & 4.175 & 3.660 & 3.605 & 3.475 & 3.420 \\
\hline $5 / 9 / 94$ & $13 d$ & 0.00 & 0.00 & 0.00 & 0.00 & 0.00 & 0.00 & 0.000 & 0.000 & 0.000 & 0.000 & 0.000 & 0.000 \\
\hline $5 / 9 / 94$ & $13 e$ & 2.15 & 1.95 & 1.90 & 1.85 & 1.85 & 1.90 & 1.860 & 1.655 & 1.545 & 1.760 & 1.755 & 1.690 \\
\hline $5 / 9 / 94$ & $13 f$ & 2.50 & 2.40 & 2.35 & 2.45 & 2.35 & 2.35 & 2.370 & 2.185 & 2.115 & 2.095 & 2.080 & 2.080 \\
\hline $5 / 9 / 94$ & $\overline{13 g}$ & 2.90 & 2.80 & 2.65 & 2.65 & 2.60 & 2.60 & 3.135 & 2.760 & 2.580 & 2.565 & 2.525 & 2.485 \\
\hline $5 / 9 / 94$ & $13 \mathrm{~h}$ & 4.65 & 3.90 & 3.80 & 3.55 & 3.55 & 3.55 & 5.385 & \begin{tabular}{|l|}
4.255 \\
\end{tabular} & 3.735 & 3.780 & 3.525 & 3.425 \\
\hline $5 / 11 / 94$ & $13 i$ & 0.00 & 0.00 & 0.00 & 0.00 & 0.00 & 0.00 & 0.000 & 0.000 & 0.000 & 0.000 & 0.000 & 0.000 \\
\hline $5 / 11 / 94$ & $13 j$ & 1.75 & 1.90 & 1.75 & 1.75 & 1.70 & 1.70 & 1.635 & 1.500 & 1.375 & 1.325 & 1.575 & 1.475 \\
\hline $5 / 11 / 94$ & $13 k$ & 2.40 & 2.40 & 2.30 & 2.25 & 2.05 & 2.40 & 2.060 & 1.865 & 1.955 & 1.905 & 1.820 & 1.950 \\
\hline $5 / 11 / 94$ & 131 & 3.00 & 2.95 & 2.85 & 2.95 & 2.95 & 2.85 & 2.810 & 2.490 & 2.215 & 2.275 & 2.345 & 2.475 \\
\hline $5 / 11 / 94$ & $13 m$ & 4.05 & 3.90 & 3.60 & 3.65 & 3.70 & 3.70 & 4.275 & 3.915 & 3.495 & 3.445 & 3.435 & 3.315 \\
\hline
\end{tabular}


Data Summary

\begin{tabular}{|c|c|c|c|c|c|c|c|c|c|c|c|c|c|}
\hline DATE & EXP & $\mathrm{AH} 2$ & $\mathrm{AH3}$ & $\overline{\mathrm{AH} 4}$ & $\mathrm{AH5}$ & AH6 & $\mathrm{AH7}$ & $\mathrm{S} 2 \mathrm{~W}$ & S3W & S4W & S5W & S6W & STW \\
\hline $5 / 11 / 94$ & $13 n$ & 1.15 & 1.10 & 1.25 & 1.20 & 1.35 & 1.25 & 0.940 & 0.965 & 1.155 & 1.015 & 1.015 & 0.850 \\
\hline $5 / 11 / 94$ & 130 & 2.15 & 2.10 & 2.15 & 2.15 & 2.05 & 1.95 & 1.545 & 1.560 & 1.485 & 1.620 & 1.415 & 1.375 \\
\hline $5 / 11 / 94$ & $13 p$ & 2.50 & 2.30 & 2.30 & 2.30 & 2.35 & 2.45 & 2.080 & 2.090 & 1.825 & 2.055 & 1.860 & 2.210 \\
\hline $5 / 11 / 94$ & $13 q$ & 2.90 & 2.80 & 2.85 & 2.90 & 2.90 & 2.85 & 2.865 & 2.415 & 2.255 & 2.370 & 2.430 & 2.415 \\
\hline $5 / 11 / 94$ & $13 r$ & 3.90 & 3.65 & 3.60 & 3.65 & 3.75 & 3.65 & 4.180 & 3.710 & 3.435 & 3.400 & 3.320 & 3.245 \\
\hline $5 / 13 / 94$ & $13 \mathrm{~s}$ & 3.80 & 3.75 & 3.80 & 3.90 & 4.00 & 4.05 & 3.735 & 3.520 & 3.370 & 3.550 & 3.490 & 3.460 \\
\hline $5 / 13 / 94$ & $13 t$ & 3.00 & 3.15 & 3.10 & 3.10 & 3.20 & 3.10 & 2.665 & 2.665 & 2.435 & 2.530 & 2.830 & 2.745 \\
\hline $5 / 13 / 94$ & $13 u$ & 2.65 & 2.65 & 2.65 & 2.65 & 2.65 & 2.60 & 2.075 & 2.165 & 2.070 & 2.160 & 2.130 & 2.065 \\
\hline $5 / 13 / 94$ & $13 v$ & 2.25 & 2.25 & 2.25 & 2.25 & 2.25 & 2.15 & 1.580 & 1.565 & 1.650 & 1.595 & 1.535 & 1.555 \\
\hline $5 / 13 / 94$ & $13 w$ & 1.25 & 1.25 & 1.25 & 1.35 & 1.45 & 4.00 & 0.830 & 0.840 & 0.750 & 0.775 & 0.740 & 0.680 \\
\hline $5 / 13 / 94$ & $13 x$ & 3.95 & 3.95 & 4.05 & 4.05 & 4.05 & 4.15 & 3.295 & 3.135 & 3.055 & 3.115 & 3.270 & 3.135 \\
\hline $5 / 13 / 94$ & $13 y$ & 2.85 & 2.90 & 3.00 & 3.05 & 3.05 & 2.95 & 2.550 & 2.495 & 2.385 & 2.530 & 2.650 & 2.260 \\
\hline $5 / 13 / 94$ & $13 z$ & 2.55 & 2.50 & 2.50 & 2.45 & 2.50 & 2.50 & 2.005 & 2.110 & 1.885 & 1.945 & 2.170 & 1.990 \\
\hline $5 / 16 / 94$ & $14 a$ & 2.20 & 2.10 & 2.10 & 2.10 & 2.10 & 2.10 & 1.485 & 1.495 & 1.400 & 1.405 & 1.485 & 1.465 \\
\hline $5 / 16 / 94$ & $14 b$ & 1.15 & 1.25 & 1.35 & 1.65 & 4.50 & 6.25 & 0.760 & 0.780 & 0.635 & 0.480 & 0.165 & 0.110 \\
\hline $5 / 16 / 94$ & $14 c$ & 3.90 & 4.10 & 4.10 & 4.25 & 4.30 & 4.30 & 3.260 & 3.215 & 3.140 & 3.220 & 3.380 & 3.375 \\
\hline $5 / 16 / 94$ & $14 d$ & 2.80 & 3.00 & 3.05 & 3.05 & 3.10 & 3.00 & 2.505 & 2.400 & 2.365 & 2.490 & 2.415 & 2.365 \\
\hline $5 / 16 / 94$ & $14 \mathrm{e}$ & 3.05 & 3.15 & 3.25 & 3.25 & 2.95 & 2.95 & 1.945 & 2.025 & 1.895 & 1.970 & 1.958 & 1.920 \\
\hline $5 / 16 / 94$ & $14 \mathrm{f}$ & 2.10 & 2.20 & 2.20 & 2.20 & 2.20 & 2.15 & 1.440 & 1.415 & 1.315 & 1.360 & 1.335 & 1.345 \\
\hline $5 / 17 / 94$ & $14 \mathrm{~g}$ & 2.20 & $4: 50$ & 11.50 & 15.00 & 21.50 & 23.50 & 0.435 & 0.100 & 0.098 & 0.089 & 0.085 & 0.834 \\
\hline $5 / 17 / 94$ & $14 \mathrm{~h}$ & 3.85 & 4.10 & 4.10 & 4.20 & 4.20 & 4.20 & 3.065 & 3.125 & 2.870 & 3.065 & 3.100 & 3.045 \\
\hline $5 / 17 / 94$ & $14 i$ & 3.10 & 3.30 & 3.30 & 3.45 & 3.50 & 3.55 & 2.450 & 2.410 & 2.205 & 2.250 & 2.160 & 2.070 \\
\hline $5 / 17 / 94$ & $14 j$ & 3.55 & 3.55 & 3.50 & 3.55 & 3.60 & 3.70 & 1.925 & 1.865 & 1.560 & 1.480 & 1.295 & 1.125 \\
\hline $5 / 17 / 94$ & $14 \mathrm{k}$ & 2.40 & 2.90 & 4.25 & 5.15 & 6.50 & 7.25 & 1.275 & 0.985 & 0.690 & 0.565 & 0.495 & 0.460 \\
\hline $5 / 17 / 94$ & 141 & 2.00 & 5.50 & 9.50 & 11.50 & 15.75 & 17.00 & 0.390 & 0.201 & 0.158 & 0.141 & 0.126 & 0.121 \\
\hline $5 / 17 / 94$ & $14 m$ & 3.60 & 3.80 & 3.80 & 3.80 & 3.80 & 3.80 & 2.875 & 3.135 & 2.845 & 2.900 & 2.830 & 2.865 \\
\hline $5 / 20 / 94$ & $14 n$ & 3.15 & 3.25 & 3.20 & 3.20 & 3.15 & 3.15 & 2.535 & 2.505 & 2.340 & 2.230 & 2.270 & 2.440 \\
\hline $5 / 20 / 94$ & 140 & 3.05 & 3.15 & 2.65 & 2.70 & 2.65 & 2.60 & 1.885 & 1.965 & 1.845 & 1.860 & 1.910 & 1.970 \\
\hline $5 / 20 / 94$ & $14 p$ & 2.15 & 2.15 & 2.10 & 2.15 & 2.15 & 2.00 & 1.420 & 1.415 & 1.325 & 1.310 & 1.295 & 1.335 \\
\hline $5 / 20 / 94$ & $14 q$ & 1.15 & 1.20 & 1.25 & 1.25 & 1.25 & 1.25 & 0.785 & 0.895 & 0.825 & 0.695 & 0.935 & 0.775 \\
\hline $5 / 20 / 94$ & $14 r$ & 3.30 & 3.55 & 3.60 & 3.50 & 3.60 & 3.65 & 3.030 & 3.035 & 2.845 & 3.090 & 3.190 & 3.095 \\
\hline $5 / 20 / 94$ & $14 s$ & 2.90 & 3.00 & 3.00 & 3.05 & 3.05 & 3.00 & 2.510 & 2.440 & 2.295 & 2.330 & 2.310 & 2.290 \\
\hline $5 / 20 / 94$ & $14 t$ & 2.50 & 2.95 & 2.70 & 2.95 & 2.95 & 3.05 & 1.945 & 1.880 & 1.745 & 1.670 & 1.570 & 1.505 \\
\hline $5 / 23 / 94$ & $14 u$ & 5.25 & 8.50 & 12.25 & 17.50 & 20.00 & 20.75 & 0.255 & 0.145 & 0.115 & 0.110 & 0.095 & 0.085 \\
\hline $5 / 23 / 94$ & $14 v$ & 3.90 & 3.65 & 3.65 & 3.90 & 4.25 & 4.25 & 3.020 & 2.995 & 2.840 & 2.915 & 2.830 & 2.760 \\
\hline $5 / 23 / 94$ & $14 w$ & 2.90 & 3.20 & 3.30 & 3.50 & 3.60 & 3.85 & 1.790 & 1.920 & 2.095 & 2.140 & 2.270 & 2.460 \\
\hline $5 / 23 / 94$ & $14 x$ & 3.10 & 3.20 & 3.75 & 4.00 & 4.40 & 5.00 & 1.925 & 1.645 & 1.395 & 1.220 & 1.035 & 0.900 \\
\hline $5 / 23 / 94$ & $14 y$ & 2.25 & 3.00 & 4.25 & 5.75 & 6.00 & 7.25 & 1.150 & 0.800 & 0.580 & 0.505 & 0.460 & 0.425 \\
\hline $5 / 23 / 94$ & 142 & 4.00 & 5.25 & 6.50 & 10.00 & 20.00 & 21.50 & 0.205 & 0.135 & 0.100 & 0.095 & 0.085 & 0.075 \\
\hline $5 / 27 / 94$ & $15 a$ & 3.80 & 3.85 & 3.85 & 4.05 & 4.30 & 4.75 & 2.960 & 2.750 & 2.480 & 2.315 & 2.120 & 1.955 \\
\hline $5 / 27 / 94$ & $15 b$ & 3.20 & 3.70 & 3.90 & 4.35 & 5.15 & 5.50 & 2.300 & 1.990 & 1.640 & 1.410 & 1.245 & 1.130 \\
\hline $5 / 30 / 94$ & $15 c$ & 3.25 & 3.55 & 4.75 & 6.75 & 7.75 & 10.25 & 1.580 & 1.145 & 0.895 & 0.775 & 0.685 & 0.635 \\
\hline $5 / 30 / 94$ & $15 d$ & 3.50 & 4.75 & 6.50 & 9.50 & 16.50 & 19.50 & 0.775 & 0.520 & 0.420 & 0.375 & 0.345 & 0.325 \\
\hline $5 / 30 / 94$ & $15 \mathrm{e}$ & 5.50 & 9.00 & 14.50 & 16.00 & 17.00 & 26.00 & 0.145 & 0.100 & 0.075 & 0.070 & 0.055 & 0.055 \\
\hline $5 / 30 / 94$ & $15 f$ & 3.70 & 4.15 & 4.15 & 4.55 & 5.40 & 5.75 & 2.565 & 2.270 & 1.950 & 1.775 & 1.590 & 1.465 \\
\hline $5 / 30 / 94$ & $15 \mathrm{~g}$ & 3.35 & 3.85 & 4.25 & 5.15 & 6.75 & 7.00 & 2.255 & 1.805 & 1.440 & 1.255 & 1.105 & 1.010 \\
\hline $5 / 30 / 94$ & $15 \mathrm{~h}$ & 3.20 & 4.20 & 5.40 & 6.75 & 9.50 & 11.75 & 1.575 & 1.150 & 0.920 & 0.805 & 0.730 & 0.685 \\
\hline $5 / 30 / 94$ & $15 i$ & 3.75 & 6.50 & 8.25 & 14.00 & 16.00 & 22.00 & 0.815 & 0.575 & 0.475 & 0.425 & 0.395 & 0.370 \\
\hline $8 / 16 / 94$ & $17 d$ & 0.00 & 0.00 & 0.00 & 0.00 & 0.00 & 0.00 & & & & & & \\
\hline $8 / 16 / 94$ & $17 \mathrm{e}$ & 0.00 & 0.00 & 0.00 & 0.00 & 0.00 & 0.00 & & & & & & \\
\hline
\end{tabular}




\begin{tabular}{|c|c|c|c|c|c|c|c|c|c|c|c|c|c|}
\hline DATE & EXP & $\mathrm{AH} 2$ & $\mathrm{AH} 3$ & $\mathrm{AH} 4$ & AH5 & $\overline{\mathrm{AH}} 6$ & AH7 & S2W & S3W & S4W & S5W & S6W & S7W \\
\hline $8 / 17 / 94$ & $17 f$ & 0.00 & 0.00 & 0.00 & 0.00 & 0.00 & 0.00 & & & & & & \\
\hline $8 / 17 / 94$ & $17 g$ & 0.00 & 0.00 & 0.00 & 0.00 & 0.00 & 0.00 & & & & & & \\
\hline $8 / 18 / 94$ & $17 \mathrm{~h}$ & 6.50 & 8.00 & 13.25 & 21.00 & 25.00 & 28.00 & 0.000 & 0.000 & 0.000 & 0.000 & 0.000 & 0.000 \\
\hline $8 / 18 / 94$ & $17 j$ & 0.00 & 0.00 & 0.00 & \begin{tabular}{|l|}
0.00 \\
\end{tabular} & 0.00 & 0.00 & & & & & & \\
\hline $8 / 18 / 94$ & $17 k$ & 0.00 & 0.00 & 0.00 & 0.00 & 0.00 & 0.00 & & & & & & \\
\hline $9 / 8 / 94$ & 171 & 0.00 & 0.00 & 0.00 & 0.00 & 0.00 & 0.00 & & & & & & \\
\hline $9 / 9 / 94$ & $17 m$ & 3.25 & 3.35 & 3.35 & 3.25 & 3.35 & 3.35 & 2.570 & 2.605 & 2.490 & 2.575 & 2.540 & 2.535 \\
\hline $9 / 12 / 94$ & $17 n$ & 0.00 & 0.00 & 0.00 & 0.00 & 0.00 & 0.00 & 2.540 & 2.545 & 2.505 & 2.510 & 2.480 & 2.490 \\
\hline $9 / 12 / 94$ & 170 & 0.00 & 0.00 & 0.00 & 0.00 & 0.00 & 0.00 & 2.530 & 2.485 & 2.435 & 2.505 & 2.510 & 2.565 \\
\hline $9 / 12 / 94$ & $17 p$ & 3.45 & 3.35 & 3.35 & 3.25 & 3.35 & 3.35 & 2.505 & 2.515 & 2.465 & 2.455 & 0.000 & 2.525 \\
\hline $9 / 12 / 94$ & $17 q$ & 3.35 & 3.35 & 3.35 & 3.35 & 3.45 & 3.35 & 2.580 & 2.565 & 2.485 & 2.535 & 2.535 & 2.580 \\
\hline $9 / 13 / 94$ & $17 r$ & 3.35 & 3.45 & 3.35 & 3.45 & 3.45 & 3.45 & 2.505 & 2.530 & 2.460 & 2.470 & 2.570 & 2.500 \\
\hline $9 / 13 / 94$ & $17 \mathrm{~s}$ & 3.35 & 3.45 & 3.35 & 3.45 & 3.45 & 3.35 & 2.470 & 2.540 & 2.435 & 2.475 & 2.480 & 2.460 \\
\hline $9 / 13 / 94$ & $17 \mathrm{t}$ & 3.35 & 3.45 & 3.45 & 3.35 & 3.45 & 3.45 & 2.485 & 2.485 & 2.480 & 2.505 & 2.470 & 2.505 \\
\hline $9 / 14 / 94$ & $17 u$ & 3.45 & 3.45 & 3.45 & 3.45 & 3.45 & 3.45 & 2.435 & 2.500 & 2.450 & 2.435 & 2.460 & 2.430 \\
\hline $9 / 14 / 94$ & $17 v$ & 3.35 & 3.35 & 3.35 & 3.45 & 3.45 & 3.35 & 2.450 & 2.445 & 2.425 & 2.430 & 0.000 & 2.430 \\
\hline $9 / 14 / 94$ & $17 w$ & 3.45 & 3.55 & 3.45 & 3.45 & 3.45 & 3.45 & 2.410 & 2.410 & 2.380 & 2.386 & 2.335 & 2.405 \\
\hline $9 / 14 / 94$ & $17 x$ & 3.65 & 3.65 & 3.65 & 3.65 & 3.55 & 3.55 & 2.240 & 2.250 & 2.140 & 2.210 & 2.190 & 2.190 \\
\hline $9 / 14 / 94$ & $17 y$ & 4.05 & 3.90 & 3.90 & 3.90 & 3.95 & 3.85 & 2.165 & 2.100 & 2.070 & 2.065 & 2.015 & 2.045 \\
\hline $9 / 14 / 94$ & 172 & 3.75 & 3.75 & 3.75 & 3.65 & 3.75 & 3.75 & 2.285 & 2.280 & 2.230 & 2.245 & 2.215 & 2.275 \\
\hline $9 / 14 / 94$ & $18 a$ & 3.65 & 3.75 & 3.65 & 3.65 & 3.65 & 3.65 & 2.140 & 2.090 & 2.070 & 2.060 & 2.020 & 2.050 \\
\hline $9 / 14 / 94$ & $18 \mathrm{~b}$ & 3.75 & 3.75 & 3.85 & 3.75 & 3.75 & 3.75 & 2.180 & 2.160 & 2.130 & 2.145 & 2.120 & 2.175 \\
\hline $9 / 14 / 94$ & $18 \mathrm{c}$ & 3.85 & 3.85 & 3.85 & 3.75 & 3.75 & 3.75 & 2.130 & 2.090 & 2.030 & 2.055 & 2.000 & 2.030 \\
\hline $9 / 14 / 94$ & $18 d$ & 3.75 & 3.85 & 3.75 & 3.75 & 3.75 & 3.75 & 1.970 & 1.955 & 1.995 & 1.990 & 2.075 & 2.100 \\
\hline $9 / 25 / 94$ & & 0.00 & 0.00 & 0.00 & 0.00 & 0.00 & 0.00 & & & & & & \\
\hline $10 / 6 / 94$ & $18 \mathrm{k}$ & 0.00 & 0.00 & 0.00 & 0.00 & 0.00 & 0.00 & & & & & & \\
\hline $10 / 6 / 94$ & 181 & 0.00 & 0.00 & 0.00 & 0.00 & 0.00 & 0.00 & & & & & & \\
\hline $10 / 7 / 94$ & $18 \mathrm{~m}$ & 0.00 & 0.00 & 0.00 & 0.00 & 0.00 & 0.00 & & & & & & \\
\hline $10 / 7 / 94$ & $18 n$ & 0.00 & 0.00 & 0.00 & 0.00 & 0.00 & 0.00 & & & & & & \\
\hline $10 / 10 / 94$ & 180 & 0.00 & 0.00 & 0.00 & 0.00 & 0.00 & 0.00 & & & & & & \\
\hline $10 / 10 / 94$ & $18 p$ & 0.00 & 0.00 & 0.00 & 0.00 & 0.00 & 0.00 & & & & & & \\
\hline $10 / 10 / 94$ & & 3.05 & 2.95 & 2.75 & 2.80 & 2.80 & 2.75 & 3.625 & 3.335 & 3.160 & 3.185 & 2.905 & 3.070 \\
\hline $10 / 10 / 94$ & & 4.45 & 4.35 & 3.95 & 3.75 & 3.45 & 2.95 & 5.760 & 5.240 & 4.735 & 4.485 & 4.025 & 3.205 \\
\hline $10 / 11 / 94$ & & 3.55 & 4.05 & 4.05 & 4.15 & 4.25 & 4.15 & 4.590 & 4.755 & 4.715 & 4.985 & 4.970 & 4.605 \\
\hline $10 / 14 / 94$ & $18 q$ & 0.00 & 0.00 & 0.00 & 0.00 & 0.00 & 0.00 & & & & & & \\
\hline $10 / 25 / 94$ & $18 \mathrm{~s}$ & 0.00 & 0.00 & 0.00 & 0.00 & 0.00 & 0.00 & & & & & & \\
\hline $10 / 26 / 94$ & $18 t$ & 0.00 & 0.00 & 0.00 & 0.00 & 0.00 & 0.00 & & & & & & \\
\hline
\end{tabular}




\begin{tabular}{|c|c|c|c|c|c|c|c|c|c|c|c|c|c|}
\hline DATE & EXP & AVF2 & AVF3 & AVF4 & AVF5 & AVF6 & AVF7 & S2V & S3V & S4V & S5V & S6V & S7V \\
\hline $1 / 19 / 93$ & $00 b$ & 0.64 & 0.50 & 0.52 & 0.55 & 0.38 & 0.36 & 5.08 & 7.14 & 8.23 & 9.53 & 12.01 & 12.23 \\
\hline $1 / 19 / 93$ & $00 c$ & 0.52 & 0.51 & 0.57 & 0.47 & 0.40 & 0.44 & 5.01 & 6.29 & 6.44 & 9.22 & 11.13 & 11.42 \\
\hline $1 / 19 / 93$ & ood & 0.62 & 0.59 & 0.59 & 0.60 & 0.53 & 0.56 & 3.94 & 4.90 & 5.58 & 6.42 & 8.14 & 8.02 \\
\hline $1 / 19 / 93$ & $00 \mathrm{e}$ & 0.51 & 0.58 & 0.53 & 0.52 & 0.46 & 0.53 & 4.34 & 4.89 & 5.47 & 6.03 & 7.10 & 6.76 \\
\hline $1 / 19 / 93$ & oof & 0.00 & 0.00 & 0.00 & 0.00 & 0.00 & 0.00 & 0.00 & 0.00 & 0.00 & 0.00 & 0.00 & 0.00 \\
\hline $1 / 21 / 93$ & $00 \mathrm{~g}$ & 0.31 & 0.24 & 0.18 & 0.16 & 0.11 & 0.10 & 7.04 & 9.01 & 10.65 & 11.61 & 14.39 & 13.56 \\
\hline $1 / 21 / 93$ & OOh & 0.34 & 0.21 & 0.12 & 0.10 & 0.05 & 0.12 & 6.43 & 9.81 & 11.08 & 13.02 & 25.01 & 10.76 \\
\hline $1 / 25 / 93$ & $00 \mathrm{i}$ & 0.52 & 0.54 & 0.50 & 0.49 & 0.43 & 0.48 & 10.94 & 12.23 & 13.67 & 15.07 & 17.76 & 16.95 \\
\hline $1 / 27 / 93$ & $00 \mathrm{j}$ & 0.55 & 0.53 & 0.55 & 0.54 & 0.53 & 0.54 & 6.45 & 7.97 & 8.88 & 10.31 & 11.79 & 12.38 \\
\hline $1 / 29 / 93$ & 00k & 0.53 & 0.51 & 0.59 & 0.46 & 0.41 & 0.45 & 6.50 & 8.16 & 8.34 & 11.95 & 14.40 & 14.80 \\
\hline $1 / 31 / 93$ & 001 & 0.28 & 0.21 & 0.16 & 0.13 & 0.10 & 0.10 & 6.70 & 9.03 & 10.74 & 11.65 & 13.93 & $\overline{13.14}$ \\
\hline $1 / 31 / 93$ & $00 \mathrm{~m}$ & 0.56 & 0.47 & 0.46 & 0.42 & 0.37 & 0.37 & 6.54 & 8.72 & 10.31 & 11.95 & 13.89 & 14.23 \\
\hline $2 / 10 / 93$ & $00 n$ & 0.33 & 0.25 & 0.21 & 0.16 & 0.12 & 0.12 & 5.97 & 7.17 & 8.39 & 9.08 & 11.22 & 10.62 \\
\hline $2 / 10 / 93$ & 000 & 0.33 & 0.26 & 0.20 & 0.17 & 0.12 & 0.12 & 8.00 & 9.62 & 11.27 & 12.51 & 15.24 & 14.68 \\
\hline $2 / 11 / 93$ & $00 p$ & 0.43 & 0.49 & 0.50 & 0.47 & 0.46 & 0.44 & 4.55 & 5.84 & 7.26 & 8.41 & 9.38 & 10.94 \\
\hline $2 / 11 / 93$ & $00 \mathrm{q}$ & 0.43 & 0.49 & 0.49 & 0.50 & 0.44 & 0.44 & 4.62 & 5.83 & 7.29 & 8.01 & 9.97 & 10.60 \\
\hline $2 / 11 / 93$ & $00 r$ & 0.49 & 0.71 & 1.06 & 1.20 & 1.00 & 1.05 & 4.22 & 4.62 & 5.57 & 6.23 & 7.65 & 8.03 \\
\hline $2 / 12 / 93$ & $00 \mathrm{~s}$ & 0.51 & 0.88 & 1.36 & 1.60 & 1.54 & 1.70 & 4.89 & 5.29 & 6.17 & 6.75 & 8.15 & 8.39 \\
\hline $2 / 12 / 93$ & $00 \mathrm{t}$ & 0.78 & 0.82 & 0.82 & 0.84 & 0.78 & 0.86 & 8.56 & 8.48 & 9.12 & 9.40 & 10.44 & 10.34 \\
\hline $2 / 12 / 93$ & $00 u$ & 0.93 & 0.76 & 0.76 & 0.75 & 0.71 & 0.77 & 8.12 & 8.49 & 9.12 & 9.40 & 10.43 & 10.34 \\
\hline $2 / 23 / 93$ & $00 \mathrm{v}$ & 0.50 & 0.48 & 0.46 & 0.47 & 0.47 & 0.47 & 5.62 & 6.11 & 6.34 & 6.34 & 6.24 & 6.22 \\
\hline $2 / 24 / 93$ & 00w & 0.37 & 0.37 & 0.38 & 0.41 & 0.40 & 0.39 & 0.73 & 0.75 & 0.76 & 0.70 & 0.72 & 0.73 \\
\hline $2 / 24 / 93$ & $00 x$ & 0.40 & 0.37 & 0.36 & 0.36 & 0.35 & 0.35 & 1.83 & 1.96 & 2.04 & 2.03 & 2.11 & 2.11 \\
\hline $2 / 24 / 93$ & $00 y$ & 0.43 & 0.40 & 0.40 & 0.39 & 0.38 & 0.38 & 2.66 & 2.78 & 2.78 & 2.84 & 2.92 & 2.91 \\
\hline $2 / 24 / 93$ & $00 z$ & 0.47 & 0.42 & 0.41 & 0.40 & 0.39 & 0.38 & 3.57 & 3.70 & 3.76 & 3.78 & 3.88 & 3.89 \\
\hline $3 / 2 / 93$ & $01 a$ & 0.05 & 0.02 & 0.01 & 0.01 & 0.01 & 0.01 & 2.30 & 2.96 & 3.18 & 4.06 & 4.33 & 4.35 \\
\hline $3 / 2 / 93$ & $01 b$ & 0.25 & 0.14 & 0.09 & 0.05 & 0.04 & 0.03 & 2.63 & 3.65 & 4.11 & 4.56 & 4.84 & 5.14 \\
\hline $3 / 2 / 93$ & $01 c$ & 0.35 & 0.29 & 0.23 & 0.18 & 0.12 & 0.08 & 3.68 & 4.25 & 5.02 & 6.06 & 6.93 & 7.39 \\
\hline $3 / 2 / 93$ & $01 d$ & 0.43 & 0.35 & 0.30 & 0.24 & 0.21 & 0.19 & 3.88 & 4.28 & 4.75 & 5.11 & 5.77 & 6.37 \\
\hline $3 / 2 / 93$ & $01 \mathrm{e}$ & 0.56 & 0.46 & 0.43 & 0.42 & 0.40 & 0.38 & 5.79 & 6.06 & 6.48 & 6.71 & 6.86 & 7.06 \\
\hline $3 / 2 / 93$ & $01 \mathrm{~g}$ & 0.23 & 0.15 & 0.11 & 0.08 & 0.07 & 0.06 & 4.07 & 4.75 & 5.27 & 5.57 & 5.74 & 5.71 \\
\hline $3 / 3 / 93$ & $01 \mathrm{~h}$ & 0.31 & 0.28 & 0.24 & 0.20 & 0.17 & 0.15 & 4.93 & 5.41 & 5.92 & 6.43 & 7.10 & 7.56 \\
\hline $3 / 3 / 93$ & $01 f$ & 0.05 & 0.03 & 0.03 & 0.02 & 0.02 & 0.01 & 3.07 & 2.95 & 2.91 & 3.16 & 3.01 & 3.29 \\
\hline $3 / 4 / 93$ & $01 k$ & 0.47 & 0.43 & 0.45 & 0.48 & 0.47 & 0.45 & 4.06 & 4.38 & 4.19 & 3.92 & 3.94 & 4.13 \\
\hline $3 / 9 / 93$ & $01 i$ & 0.44 & 0.42 & 0.45 & 0.40 & 0.35 & 0.46 & 1.94 & 2.03 & 1.83 & 2.07 & 2.32 & 1.77 \\
\hline $3 / 9 / 93$ & 01j & 0.46 & 0.45 & 0.44 & 0.40 & 0.45 & 0.45 & 2.55 & 2.37 & 2.43 & 2.66 & 2.42 & 2.40 \\
\hline $3 / 9 / 93$ & 011 & 0.57 & 0.49 & 0.00 & 0.48 & 0.00 & 0.51 & 5.15 & 6.01 & 0.00 & 6.18 & 0.00 & 5.68 \\
\hline $3 / 9 / 93$ & $01 \mathrm{~m}$ & 0.06 & 0.04 & 0.04 & $\overline{0.04}$ & 0.03 & 0.03 & 2.27 & 2.60 & 2.65 & 2.67 & 2.65 & 2.67 \\
\hline $3 / 9 / 93$ & $01 n$ & 0.33 & 0.42 & 0.41 & 0.40 & 0.52 & 0.41 & 2.35 & 2.17 & 2.36 & 2.42 & 1.88 & 2.38 \\
\hline $3 / 9 / 93$ & 010 & 0.41 & 0.41 & 0.34 & 0.37 & 0.36 & 0.43 & 3.89 & 3.94 & 4.67 & 4.40 & 4.53 & 3.72 \\
\hline $3 / 15 / 93$ & $01 p$ & 0.03 & 0.02 & 0.01 & 0.00 & 0.00 & 0.00 & 2.60 & 2.54 & 2.06 & 4.25 & 4.63 & 4.69 \\
\hline $3 / 15 / 93$ & 019 & 0.21 & 0.09 & 0.06 & 0.04 & 0.03 & 0.02 & 3.06 & 4.32 & 4.02 & 4.51 & 4.43 & 4.69 \\
\hline $3 / 15 / 93$ & $01 \mathrm{r}$ & 0.31 & 0.23 & 0.16 & 0.10 & 0.08 & 0.06 & 3.61 & 4.52 & 5.64 & 5.82 & 6.39 & 7.10 \\
\hline $3 / 15 / 93$ & $01 \mathrm{~s}$ & 0.45 & 0.35 & 0.30 & 0.24 & 0.17 & 0.13 & 3.79 & 4.61 & 5.43 & 6.20 & 6.97 & 7.72 \\
\hline $3 / 15 / 93$ & $01 t$ & 0.47 & 0.43 & 0.40 & 0.39 & 0.35 & 0.35 & 5.58 & 6.10 & 6.53 & 6.71 & 7.24 & 7.35 \\
\hline $3 / 15 / 93$ & 014 & 0.04 & 0.03 & 0.02 & 0.02 & 0.02 & 0.01 & 4.14 & 3.88 & 3.25 & 3.48 & 4.21 & 4.76 \\
\hline $3 / 15 / 93$ & $01 v$ & 0.21 & 0.14 & 0.10 & 0.07 & 0.06 & 0.04 & 4.48 & 5.69 & 6.79 & 7.28 & 7.41 & 7.63 \\
\hline $3 / 15 / 93$ & $01 w$ & 0.27 & 0.21 & 0.17 & 0.13 & 0.10 & 0.10 & 5.22 & 6.02 & 7.03 & 7.73 & 8.44 & 7.73 \\
\hline $3 / 18 / 93$ & $01 x$ & 0.58 & 0.53 & 0.55 & 0.51 & 0.52 & 0.49 & 0.63 & 0.70 & 0.73 & 0.74 & 0.72 & 0.80 \\
\hline $3 / 18 / 93$ & $01 y$ & 0.00 & 0.00 & 0.00 & 0.00 & 0.00 & 0.00 & 0.00 & 0.00 & 0.00 & 0.00 & 0.00 & 0.00 \\
\hline
\end{tabular}




\begin{tabular}{|c|c|c|c|c|c|c|c|c|c|c|c|c|c|}
\hline DATE & EXP & AVF2 & AVF3 & AVF4 & AVF5 & AVF6 & AVF7 & $\mathrm{S} 2 \mathrm{~V}$ & S3V & $S 4 \mathrm{~V}$ & S5V & S6V & S7V \\
\hline $3 / 18 / 93$ & 012 & 0.56 & 0.58 & 0.59 & 0.54 & 0.53 & 0.51 & 1.25 & 1.58 & 1.67 & 1.71 & 1.74 & 1.88 \\
\hline $3 / 18 / 93$ & $02 a$ & 0.55 & 0.60 & 0.60 & 0.56 & 0.51 & 0.43 & 0.63 & 0.62 & 0.58 & 0.62 & 0.68 & 0.80 \\
\hline $3 / 18 / 93$ & $02 b$ & 0.61 & 0.56 & 0.56 & 0.53 & 0.54 & 0.57 & 1.08 & 1.21 & 1.24 & 1.21 & 1.18 & 1.23 \\
\hline $3 / 18 / 93$ & $02 c$ & 0.59 & 0.61 & 0.63 & 0.59 & 0.59 & 0.60 & 2.02 & 2.59 & 2.76 & 2.96 & 2.94 & 2.90 \\
\hline $3 / 18 / 93$ & $02 d$ & 0.61 & 0.57 & 0.58 & 0.57 & 0.57 & 0.53 & 1.68 & 1.94 & 2.14 & 2.15 & 2.17 & 2.31 \\
\hline $3 / 19 / 93$ & $02 e$ & 0.56 & 0.58 & 0.56 & 0.54 & 0.57 & 0.57 & 1.03 & 1.30 & 1.39 & 1.44 & 1.41 & 1.47 \\
\hline $3 / 22 / 93$ & $02 f$ & 0.04 & 0.03 & 0.01 & 0.01 & 0.01 & 0.01 & 3.05 & 2.74 & 3.82 & 5.06 & 5.78 & 5.99 \\
\hline $3 / 22 / 93$ & $02 \mathrm{~g}$ & 0.13 & 0.06 & 0.04 & 0.03 & 0.02 & 0.02 & 6.37 & 6.59 & 6.95 & 6.63 & 6.34 & 7.06 \\
\hline $3 / 22 / 93$ & $02 \mathrm{~h}$ & 0.21 & 0.14 & 0.10 & 0.08 & 0.05 & 0.04 & 7.98 & 9.85 & 11.18 & 11.69 & 10.20 & 10.24 \\
\hline $3 / 22 / 93$ & $02 i$ & 0.03 & 0.01 & 0.01 & 0.00 & 0.00 & 0.00 & 2.26 & 2.45 & 3.18 & 3.78 & 4.14 & 5.25 \\
\hline $3 / 22 / 93$ & $09 z$ & 0.00 & 0.00 & 0.00 & 0.00 & 0.00 & 0.00 & 0.00 & 0.00 & 0.00 & 0.00 & 0.00 & 0.00 \\
\hline $3 / 23 / 93$ & $02 j$ & 0.07 & 0.04 & 0.03 & 0.01 & 0.01 & 0.01 & 4.63 & 4.52 & 5.14 & 5.58 & 5.54 & 5.64 \\
\hline $3 / 23 / 93$ & $02 k$ & 0.18 & 0.09 & 0.06 & 0.04 & 0.03 & 0.02 & 6.25 & 6.74 & 7.37 & 7.75 & 7.87 & 6.36 \\
\hline $3 / 23 / 93$ & $02 !$ & 0.26 & 0.17 & 0.12 & 0.07 & 0.06 & 0.05 & 6.70 & 8.60 & 10.06 & 9.60 & 8.85 & 7.67 \\
\hline $3 / 23 / 93$ & $02 m$ & 0.30 & 0.23 & 0.17 & 0.14 & 0.12 & 0.10 & 8.03 & 9.79 & 11.08 & 11.79 & 12.70 & 13.16 \\
\hline $3 / 24 / 93$ & $02 n$ & 0.03 & 0.01 & 0.01 & 0.01 & 0.00 & 0.00 & 3.75 & 4.98 & 5.90 & 5.57 & 5.35 & 5.86 \\
\hline $3 / 24 / 93$ & 020 & 0.11 & 0.05 & 0.04 & 0.02 & 0.02 & 0.02 & 5.58 & 6.18 & 6.83 & 7.23 & 6.23 & 5.74 \\
\hline $3 / 24 / 93$ & $\overline{02 p}$ & 0.23 & 0.17 & 0.12 & 0.09 & 0.07 & 0.06 & 6.95 & 8.77 & 9.68 & 10.61 & 9.83 & 8.50 \\
\hline $3 / 24 / 93$ & $02 q$ & 0.02 & 0.01 & 0.01 & 0.00 & 0.00 & 0.00 & 2.38 & 2.68 & 3.53 & 3.76 & 4.51 & 4.95 \\
\hline $3 / 24 / 93$ & $02 r$ & 0.09 & 0.04 & 0.02 & 0.01 & 0.01 & 0.01 & 4.66 & 5.74 & 6.28 & 6.99 & 6.09 & 6.66 \\
\hline $3 / 24 / 93$ & $02 s$ & 0.21 & 0.11 & 0.07 & 0.05 & 0.04 & 0.04 & 5.34 & 6.42 & 6.58 & 6.38 & 5.81 & 4.98 \\
\hline $3 / 24 / 93$ & $02 t$ & 0.31 & 0.21 & 0.15 & 0.10 & 0.08 & 0.06 & 5.53 & 7.16 & 8.56 & 8.71 & 8.04 & 7.60 \\
\hline $3 / 24 / 93$ & $02 u$ & 0.36 & 0.28 & 0.24 & 0.18 & 0.15 & 0.13 & 7.23 & 8.61 & 10.12 & 10.72 & 11.07 & 12.15 \\
\hline $3 / 24 / 93$ & $02 v$ & 0.04 & 0.01 & 0.01 & 0.00 & 0.00 & 0.00 & 1.61 & 2.71 & 4.01 & 4.69 & 5.20 & 5.89 \\
\hline $3 / 24 / 93$ & $02 w$ & 0.10 & 0.05 & 0.03 & 0.01 & 0.01 & 0.01 & 4.92 & 5.32 & 4.47 & 7.16 & 6.69 & 7.09 \\
\hline $3 / 25 / 93$ & $02 x$ & 0.19 & 0.10 & 0.06 & 0.05 & 0.03 & 0.03 & 5.78 & 6.86 & 7.19 & 7.71 & 8.01 & 7.77 \\
\hline $3 / 25 / 93$ & $02 y$ & 0.32 & 0.18 & 0.14 & 0.09 & 0.07 & 0.06 & 5.92 & 7.78 & 9.32 & 10.16 & 9.20 & 8.47 \\
\hline $3 / 25 / 93$ & 022 & 0.35 & 0.26 & 0.21 & 0.15 & 0.14 & 0.12 & 7.69 & 9.30 & 10.56 & 11.43 & 12.35 & 13.42 \\
\hline $3 / 25 / 93$ & $03 a$ & 0.03 & 0.02 & 0.02 & 0.01 & 0.00 & 0.00 & 2.62 & 2.38 & 2.38 & 4.72 & 5.72 & 5.48 \\
\hline $3 / 25 / 93$ & $03 b$ & 0.09 & 0.05 & 0.04 & 0.02 & 0.02 & 0.01 & 5.44 & 5.89 & 5.37 & 7.35 & 6.32 & 6.74 \\
\hline $3 / 25 / 93$ & $\overline{03 c}$ & 0.26 & 0.17 & 0.12 & 0.09 & 0.07 & 0.06 & 7.38 & 9.23 & 10.56 & 10.20 & 9.66 & 9.64 \\
\hline $3 / 25 / 93$ & 03d & 0.03 & 0.01 & 0.01 & 0.01 & 0.00 & 0.00 & 2.26 & 2.21 & 2.49 & 2.50 & 3.67 & 4.10 \\
\hline $3 / 25 / 93$ & $03 e$ & 0.12 & 0.05 & 0.03 & 0.02 & 0.02 & 0.02 & 4.17 & 4.68 & 5.31 & 5.90 & 5.43 & 4.89 \\
\hline $3 / 25 / 93$ & $03 f$ & 0.27 & 0.14 & 0.10 & 0.06 & 0.05 & 0.04 & 4.65 & 6.51 & 7.57 & 7.49 & 7.65 & 7.65 \\
\hline $3 / 25 / 93$ & $03 g$ & 0.36 & 0.24 & 0.19 & 0.12 & 0.09 & 0.08 & 4.93 & 6.30 & 7.98 & 8.81 & 9.47 & 9.72 \\
\hline $3 / 25 / 93$ & $03 \mathrm{~h}$ & 0.43 & 0.37 & 0.29 & 0.24 & 0.20 & 0.18 & 6.59 & 7.59 & 8.71 & 9.70 & 10.59 & 11.58 \\
\hline $3 / 26 / 93$ & $03 i$ & 0.04 & 0.03 & 0.02 & 0.01 & 0.01 & 0.01 & 2.68 & 2.42 & 2.78 & 3.98 & 4.85 & 4.63 \\
\hline $3 / 26 / 93$ & $03 j$ & 0.10 & 0.07 & 0.05 & 0.03 & 0.03 & 0.03 & 5.16 & 5.04 & 5.71 & 6.20 & 6.22 & 5.14 \\
\hline $3 / 26 / 93$ & $03 k$ & 0.27 & 0.19 & 0.15 & 0.11 & 0.08 & 0.07 & 6.29 & 7.77 & 9.08 & 9.66 & 9.27 & 8.85 \\
\hline $3 / 26 / 93$ & 031 & 0.04 & 0.02 & 0.02 & 0.01 & 0.01 & 0.00 & 1.95 & 1.98 & 1.73 & 2.18 & 2.96 & 3.52 \\
\hline $3 / 26 / 93$ & $03 m$ & 0.25 & 0.12 & 0.07 & 0.05 & 0.04 & 0.03 & 2.63 & 3.72 & 4.27 & 4.24 & 5.10 & 4.28 \\
\hline $3 / 26 / 93$ & $03 n$ & 0.35 & 0.28 & 0.21 & 0.15 & 0.11 & 0.09 & 3.18 & 3.76 & 4.61 & 5.43 & 6.54 & 6.50 \\
\hline $3 / 26 / 93$ & 030 & 0.44 & 0.38 & 0.31 & 0.27 & 0.23 & 0.20 & 3.84 & 4.22 & 4.56 & 4.82 & 5.41 & 6.04 \\
\hline $3 / 26 / 93$ & $03 p$ & 0.49 & 0.46 & 0.44 & 0.41 & 0.39 & 0.38 & 5.56 & 5.84 & 6.14 & 6.25 & 6.62 & 6.68 \\
\hline $3 / 26 / 93$ & $03 q$ & 0.04 & 0.03 & 0.02 & 0.01 & 0.01 & 0.01 & 3.00 & 2.50 & 2.82 & 3.11 & 3.31 & 2.98 \\
\hline $3 / 26 / 93$ & $03 r$ & 0.15 & 0.09 & 0.06 & 0.05 & 0.04 & 0.04 & 4.53 & 5.26 & 5.20 & 5.15 & 4.80 & 4.58 \\
\hline $3 / 26 / 93$ & $03 \mathrm{~s}$ & 0.33 & 0.29 & 0.26 & 0.23 & 0.19 & 0.15 & 4.92 & 5.38 & 6.05 & 6.55 & 6.83 & 7.87 \\
\hline $3 / 29 / 93$ & $03 t$ & 0.06 & 0.05 & 0.04 & 0.04 & 0.03 & 0.03 & 2.59 & 2.93 & 2.68 & 2.81 & 2.87 & 3.08 \\
\hline $3 / 29 / 93$ & $03 u$ & 0.44 & 0.47 & 0.49 & 0.59 & 0.39 & 0.51 & 1.80 & 1.84 & 1.79 & 1.48 & 2.22 & 1.70 \\
\hline $3 / 29 / 93$ & $03 v$ & 0.42 & 0.45 & 0.48 & 0.41 & 0.45 & 0.56 & 3.51 & 3.59 & 3.36 & 3.91 & 3.61 & 2.91 \\
\hline
\end{tabular}




\begin{tabular}{|c|c|c|c|c|c|c|c|c|c|c|c|c|c|}
\hline DATE & EXP & AVF2 & AVF3 & AVF4 & AVF5 & AVF6 & AVF7 & S2V & S3V & S4V & S5V & S6V & S7V \\
\hline $3 / 29 / 93$ & $03 w$ & 0.00 & 0.00 & 0.00 & 0.00 & 0.00 & 0.00 & 0.00 & 0.00 & 0.00 & 0.00 & 0.00 & 0.00 \\
\hline $3 / 29 / 93$ & $03 x$ & 0.43 & 0.44 & 0.42 & 0.53 & 0.41 & 0.37 & 1.49 & 1.44 & 1.52 & 1.18 & 1.55 & 1.70 \\
\hline $3 / 29 / 93$ & $03 y$ & 0.52 & 0.43 & 0.42 & 0.53 & 0.50 & 0.41 & 2.09 & 2.48 & 2.53 & 2.00 & 2.12 & 2.61 \\
\hline $3 / 29 / 93$ & $03 z$ & 0.54 & 0.43 & 0.45 & 0.45 & 0.40 & 0.46 & 2.75 & 3.41 & 3.25 & 3.30 & 3.71 & 3.20 \\
\hline $3 / 29 / 93$ & $04 a$ & 0.58 & 0.49 & 0.44 & 0.46 & 0.48 & 0.44 & 4.37 & 5.16 & 5.84 & 5.51 & 5.28 & 5.72 \\
\hline $3 / 30 / 93$ & $04 b$ & 0.54 & 0.59 & 0.56 & 0.53 & 0.48 & 0.43 & 0.46 & 0.44 & 0.42 & 0.41 & 0.45 & 0.50 \\
\hline $3 / 30 / 93$ & $04 c$ & 0.58 & 0.56 & 0.55 & 0.55 & 0.49 & 0.51 & 1.28 & 1.28 & 1.29 & 1.25 & 1.41 & 1.35 \\
\hline $3 / 30 / 93$ & 04d & 0.55 & 0.55 & 0.56 & 0.52 & 0.54 & 0.52 & 1.69 & 1.92 & 1.90 & 1.91 & 1.85 & 1.89 \\
\hline $3 / 30 / 93$ & $04 e$ & 0.57 & 0.59 & 0.56 & 0.53 & 0.55 & 0.53 & 3.05 & 3.63 & 3.93 & 4.11 & 4.09 & 4.32 \\
\hline $3 / 30 / 93$ & $04 f$ & 0.52 & 0.61 & 0.54 & 0.53 & 0.53 & 0.51 & 0.59 & 0.60 & 0.60 & 0.58 & 0.57 & 0.60 \\
\hline $3 / 30 / 93$ & $04 \mathrm{~g}$ & 0.56 & 0.56 & 0.55 & 0.54 & 0.55 & $\overline{0.51}$ & 1.63 & 1.67 & 1.71 & 1.64 & 1.58 & 1.71 \\
\hline $3 / 30 / 93$ & $04 \mathrm{~h}$ & 0.56 & 0.56 & 0.56 & 0.54 & 0.55 & 0.58 & 2.27 & 2.21 & 2.19 & 2.27 & 2.22 & 2.12 \\
\hline $3 / 30 / 93$ & $04 \hat{i}$ & 0.65 & 0.60 & 0.59 & 0.56 & 0.56 & 0.58 & 0.85 & 1.10 & 1.26 & 1.29 & 1.25 & 1.22 \\
\hline $3 / 30 / 93$ & $04 j$ & 0.53 & 0.57 & 0.58 & 0.61 & 0.63 & 0.61 & 0.90 & 1.18 & 1.23 & 1.18 & 1.13 & 1.17 \\
\hline $4 / 1 / 93$ & $04 n$ & 0.56 & 0.50 & 0.46 & 0.53 & 0.55 & 0.51 & 2.53 & 2.85 & 3.06 & 2.68 & 2.58 & 2.80 \\
\hline $4 / 1 / 93$ & 04k & 0.46 & 0.46 & $\overline{0.41}$ & 0.41 & 0.41 & 0.40 & 3.63 & 3.52 & 3.83 & 3.61 & 3.64 & 3.73 \\
\hline $4 / 1 / 93$ & 041 & 0.40 & 0.49 & 0.44 & 0.45 & 0.44 & 0.44 & 6.73 & 5.56 & 5.98 & 5.64 & 5.44 & 5.55 \\
\hline $4 / 1 / 93$ & $04 \mathrm{~m}$ & 0.53 & 0.39 & 0.38 & 0.39 & 0.39 & 0.39 & 2.87 & 3.91 & 4.01 & 3.87 & 3.90 & 3.88 \\
\hline $4 / 1 / 93$ & 040 & 0.60 & 0.59 & 0.53 & 0.53 & 0.49 & 0.51 & 3.53 & 4.24 & 4.93 & 4.97 & 5.30 & 4.97 \\
\hline $4 / 1 / 93$ & $04 p$ & 0.47 & 0.48 & 0.48 & 0.52 & 0.54 & 0.55 & 2.98 & 2.94 & 2.91 & 2.67 & 2.62 & 2.56 \\
\hline $4 / 1 / 93$ & $04 q$ & 0.56 & 0.67 & 0.68 & 0.67 & 0.64 & 0.67 & 0.42 & 0.48 & 0.47 & 0.47 & 0.49 & 0.47 \\
\hline $4 / 2 / 93$ & $04 r$ & 0.34 & 0.26 & 0.20 & 0.16 & 0.12 & 0.09 & 8.10 & 9.43 & 11.15 & 12.33 & 13.30 & 14.79 \\
\hline $4 / 5 / 93$ & $04 s$ & 0.00 & 0.00 & 0.00 & 0.00 & 0.00 & 0.00 & 0.00 & 0.00 & 0.00 & 0.00 & 0.00 & 0.00 \\
\hline $4 / 5 / 93$ & $04 t$ & 0.00 & 0.00 & 0.00 & 0.00 & 0.00 & 0.00 & 0.00 & 0.00 & 0.00 & 0.00 & 0.00 & 0.00 \\
\hline $4 / 5 / 93$ & $04 u$ & 0.00 & 0.00 & 0.00 & 0.00 & 0.00 & 0.00 & 0.00 & 0.00 & 0.00 & 0.00 & 0.00 & 0.00 \\
\hline $4 / 5 / 93$ & $04 v$ & 0.00 & 0.00 & 0.00 & 0.00 & 0.00 & 0.00 & 0.00 & 0.00 & 0.00 & 0.00 & 0.00 & 9.27 \\
\hline $4 / 5 / 93$ & $04 w$ & 0.00 & 0.00 & 0.12 & 0.00 & 0.00 & 0.06 & 0.00 & 0.00 & 0.00 & 0.00 & 0.00 & 5.36 \\
\hline $4 / 5 / 93$ & $04 x$ & 0.00 & 0.00 & 0.00 & 0.00 & 0.00 & 0.00 & 0.00 & 0.00 & 0.00 & 0.00 & 0.00 & 0.00 \\
\hline $4 / 5 / 93$ & $04 y$ & 0.53 & 0.00 & 0.57 & 0.00 & 0.55 & 0.54 & 0.83 & 0.00 & 0.00 & 0.00 & 0.75 & 0.78 \\
\hline $4 / 5 / 93$ & $04 z$ & 0.54 & 0.00 & 0.49 & 0.46 & 0.47 & 0.45 & 1.79 & 0.00 & 0.00 & 1.91 & 1.88 & 1.92 \\
\hline $4 / 5 / 93$ & $05 a$ & 0.48 & 0.51 & 0.51 & 0.53 & 0.54 & 0.51 & 2.29 & 2.24 & 2.23 & 2.14 & 2.09 & 2.23 \\
\hline $4 / 5 / 93$ & $05 b$ & 0.00 & 0.00 & 0.00 & 0.00 & 0.00 & 0.00 & 0.00 & 0.00 & 0.00 & 0.00 & 0.00 & 0.00 \\
\hline $4 / 7 / 93$ & $05 c$ & 0.46 & 0.00 & 0.00 & 0.00 & 0.40 & 0.00 & 5.93 & 0.00 & 0.00 & 0.00 & 6.72 & 0.00 \\
\hline $4 / 7 / 93$ & $05 d$ & 0.05 & 0.00 & 0.03 & 0.00 & 0.01 & 0.01 & 2.13 & 0.00 & 0.00 & 0.00 & 3.69 & 2.58 \\
\hline $4 / 7 / 93$ & $05 e$ & 0.13 & 0.10 & 0.08 & 0.07 & 0.05 & 0.05 & 4.56 & 5.14 & 4.95 & 5.25 & 4.75 & 4.50 \\
\hline $4 / 7 / 93$ & $05 f$ & 0.34 & 0.00 & 0.29 & 0.00 & 0.17 & 0.15 & 5.02 & 0.00 & 0.00 & 0.00 & 7.08 & 7.52 \\
\hline $4 / 12 / 93$ & $05 \mathrm{~g}$ & 0.54 & 0.55 & 0.50 & 0.51 & 0.51 & 0.51 & 2.00 & 2.22 & 2.29 & 2.25 & 2.23 & 2.22 \\
\hline $4 / 12 / 93$ & $05 \mathrm{~h}$ & 0.00 & 0.00 & 0.00 & 0.00 & 0.00 & 0.00 & 0.00 & 0.00 & 0.00 & 0.00 & 0.00 & 0.00 \\
\hline $6 / 14 / 93$ & $05 n$ & 0.55 & 0.52 & 0.52 & 0.50 & 0.50 & 0.47 & 3.45 & 4.28 & 4.94 & 5.10 & 5.05 & 5.23 \\
\hline $6 / 14 / 93$ & 050 & 0.56 & 0.39 & 0.37 & 0.54 & 0.57 & 0.52 & 1.80 & 2.17 & 2.32 & 2.08 & 1.97 & 1.99 \\
\hline $6 / 14 / 93$ & 05p & 0.62 & 0.46 & 0.48 & 0.48 & 0.51 & 0.44 & 2.46 & 3.06 & 3.09 & 3.03 & 2.80 & 3.00 \\
\hline $6 / 14 / 93$ & $05 q$ & 0.00 & 0.00 & 0.00 & 0.00 & 0.00 & 0.00 & 0.00 & 0.00 & 0.00 & 0.00 & 0.00 & 0.00 \\
\hline $6 / 14 / 93$ & $05 r$ & 0.00 & 0.00 & 0.00 & 0.00 & 0.00 & 0.00 & 0.00 & 0.00 & 0.00 & 0.00 & 0.00 & 0.00 \\
\hline $6 / 14 / 93$ & $05 s$ & 0.00 & 0.00 & 0.00 & 0.00 & 0.00 & 0.00 & 0.00 & 0.00 & 0.00 & 0.00 & 0.00 & 0.00 \\
\hline $6 / 15 / 93$ & $05 t$ & 0.00 & 0.00 & 0.00 & 0.00 & 0.00 & 0.00 & 0.00 & 0.00 & 0.00 & 0.00 & 0.00 & 0.00 \\
\hline $6 / 15 / 93$ & $05 u$ & 0.00 & 0.00 & 0.00 & 0.00 & 0.00 & 0.00 & 0.00 & 0.00 & 0.00 & 0.00 & 0.00 & 0.00 \\
\hline $6 / 16 / 93$ & $05 v$ & 0.00 & 0.00 & 0.00 & 0.00 & 0.00 & 0.00 & 0.00 & 0.00 & 0.00 & 0.00 & 0.00 & 0.00 \\
\hline $6 / 17 / 93$ & $05 w$ & 0.00 & 0.00 & 0.00 & 0.00 & 0.00 & 0.00 & 0.00 & 0.00 & 0.00 & 0.00 & 0.00 & 0.00 \\
\hline $6 / 17 / 93$ & $05 x$ & 0.00 & 0.00 & 0.00 & 0.00 & 0.00 & 0.00 & 0.00 & 0.00 & 0.00 & 0.00 & 0.00 & 0.00 \\
\hline $6 / 17 / 93$ & $05 y$ & 0.00 & 0.00 & 0.00 & 0.00 & 0.00 & 0.00 & 0.00 & 0.00 & 0.00 & 0.00 & 0.00 & 0.00 \\
\hline
\end{tabular}




\begin{tabular}{|c|c|c|c|c|c|c|c|c|c|c|c|c|c|}
\hline DATE & EXP & AVF2 & AVF3 & AVF4 & AVF5 & AVF6 & AVF7 & S2V & S3V & S4V & S5V & S6V & S7V \\
\hline $6 / 17 / 93$ & $05 z$ & 0.00 & 0.00 & 0.00 & 0.00 & 0.00 & 0.00 & 0.00 & 0.00 & 0.00 & 0.00 & 0.00 & 0.00 \\
\hline $6 / 28 / 93$ & $06 a$ & 0.00 & 0.00 & 0.00 & 0.00 & 0.00 & 0.00 & 0.00 & 0.00 & 0.00 & 0.00 & 0.00 & 0.00 \\
\hline $7 / 1 / 93$ & $06 b$ & 0.00 & 0.00 & 0.00 & 0.00 & 0.00 & 0.00 & 0.00 & 0.00 & 0.00 & 0.00 & 0.00 & 0.00 \\
\hline $7 / 1 / 93$ & $06 c$ & 0.00 & 0.00 & 0.00 & 0.00 & 0.00 & 0.00 & 0.00 & 0.00 & 0.00 & 0.00 & 0.00 & 0.00 \\
\hline $7 / 1 / 93$ & $06 d$ & 0.00 & 0.00 & 0.00 & 0.00 & 0.00 & 0.00 & 0.00 & 0.00 & 0.00 & 0.00 & 0.00 & 0.00 \\
\hline $7 / 1 / 93$ & $06 \mathrm{e}$ & 0.00 & 0.00 & 0.00 & 0.00 & 0.00 & 0.00 & 0.00 & 0.00 & 0.00 & 0.00 & 0.00 & 0.00 \\
\hline $7 / 6 / 93$ & $06 f$ & 0.00 & 0.00 & 0.00 & 0.00 & 0.00 & 0.00 & 0.00 & 0.00 & 0.00 & 0.00 & 0.00 & 0.00 \\
\hline $7 / 6 / 93$ & $06 \mathrm{~h}$ & 0.00 & 0.00 & 0.00 & 0.00 & 0.00 & 0.00 & 0.00 & 0.00 & 0.00 & 0.00 & 0.00 & 0.00 \\
\hline $7 / 8 / 93$ & $06 \mathrm{~g}$ & 0.00 & 0.00 & 0.00 & 0.00 & 0.00 & 0.00 & 0.00 & 0.00 & 0.00 & 0.00 & 0.00 & 0.00 \\
\hline $7 / 8 / 93$ & $06 i$ & 0.00 & 0.00 & 0.00 & 0.00 & 0.00 & 0.00 & 0.00 & 0.00 & 0.00 & 0.00 & 0.00 & 0.00 \\
\hline $7 / 8 / 93$ & $06 \mathrm{j}$ & 0.00 & 0.00 & 0.00 & 0.00 & 0.00 & 0.00 & 0.00 & 0.00 & 0.00 & 0.00 & 0.00 & 0.00 \\
\hline $7 / 8 / 93$ & $06 k$ & 0.00 & 0.00 & 0.00 & 0.00 & 0.00 & 0.00 & 0.00 & 0.00 & 0.00 & 0.00 & 0.00 & 0.00 \\
\hline $7 / 8 / 93$ & 061 & 0.00 & 0.00 & 0.00 & 0.00 & 0.00 & 0.00 & 0.00 & 0.00 & 0.00 & 0.00 & 0.00 & 0.00 \\
\hline $7 / 8 / 93$ & $06 \mathrm{~m}$ & 0.00 & 0.00 & 0.00 & 0.00 & 0.00 & 0.00 & 0.00 & 0.00 & 0.00 & 0.00 & 0.00 & 0.00 \\
\hline $7 / 8 / 93$ & $06 n$ & 0.00 & 0.00 & 0.00 & 0.00 & 0.00 & 0.00 & 0.00 & 0.00 & 0.00 & 0.00 & 0.00 & 0.00 \\
\hline $7 / 8 / 93$ & 060 & 0.00 & 0.00 & 0.00 & 0.00 & 0.00 & 0.00 & 0.00 & 0.00 & 0.00 & 0.00 & 0.00 & 0.00 \\
\hline $7 / 12 / 93$ & $06 p$ & 0.00 & 0.00 & 0.00 & 0.00 & 0.00 & 0.00 & 0.00 & 0.00 & 0.00 & 0.00 & 0.00 & 0.00 \\
\hline $7 / 12 / 93$ & $06 q$ & 0.00 & 0.00 & 0.00 & 0.00 & 0.00 & 0.00 & 0.00 & 0.00 & 0.00 & 0.00 & 0.00 & 0.00 \\
\hline $7 / 12 / 93$ & $06 r$ & 0.00 & 0.00 & 0.00 & 0.00 & 0.00 & 0.00 & 0.00 & 0.00 & 0.00 & 0.00 & 0.00 & 0.00 \\
\hline $7 / 12 / 93$ & $06 s$ & 0.00 & 0.00 & 0.00 & 0.00 & 0.00 & 0.00 & 0.00 & 0.00 & 0.00 & 0.00 & 0.00 & 0.00 \\
\hline $7 / 12 / 93$ & $06 t$ & 0.00 & 0.00 & 0.00 & 0.00 & 0.00 & 0.00 & 0.00 & 0.00 & 0.00 & 0.00 & 0.00 & 0.00 \\
\hline $7 / 12 / 93$ & $06 u$ & 0.00 & 0.00 & 0.00 & 0.00 & 0.00 & 0.00 & 0.00 & 0.00 & 0.00 & 0.00 & 0.00 & 0.00 \\
\hline $7 / 12 / 93$ & $06 v$ & 0.00 & 0.00 & 0.00 & 0.00 & 0.00 & 0.00 & 0.00 & 0.00 & 0.00 & 0.00 & 0.00 & 0.00 \\
\hline $7 / 13 / 93$ & $06 w$ & 0.00 & 0.00 & 0.00 & 0.00 & 0.00 & 0.00 & 0.00 & 0.00 & 0.00 & 0.00 & 0.00 & $\overline{0.00}$ \\
\hline $7 / 13 / 93$ & $06 x$ & 0.00 & 0.00 & 0.00 & 0.00 & 0.00 & 0.00 & 0.00 & 0.00 & 0.00 & 0.00 & 0.00 & 0.00 \\
\hline $7 / 13 / 93$ & $06 y$ & 0.00 & 0.00 & 0.00 & 0.00 & 0.00 & 0.00 & 0.00 & 0.00 & 0.00 & 0.00 & 0.00 & 0.00 \\
\hline $7 / 13 / 93$ & $06 z$ & 0.00 & 0.00 & 0.00 & 0.00 & 0.00 & 0.00 & 0.00 & 0.00 & 0.00 & 0.00 & 0.00 & 0.00 \\
\hline $7 / 17 / 93$ & $07 a$ & 0.00 & 0.00 & 0.00 & 0.00 & 0.00 & 0.00 & 0.00 & 0.00 & 0.00 & 0.00 & 0.00 & 0.00 \\
\hline $7 / 17 / 93$ & $07 \mathrm{~b}$ & 0.00 & 0.00 & 0.00 & 0.00 & 0.00 & 0.00 & 0.00 & 0.00 & 0.00 & 0.00 & 0.00 & 0.00 \\
\hline $7 / 17 / 93$ & $07 \mathrm{c}$ & 0.00 & 0.00 & 0.00 & 0.00 & 0.00 & 0.00 & 0.00 & 0.00 & 0.00 & 0.00 & 0.00 & 0.00 \\
\hline $7 / 19 / 93$ & 07d & 0.00 & 0.00 & 0.00 & 0.00 & 0.00 & 0.00 & 0.00 & 0.00 & 0.00 & 0.00 & 0.00 & 0.00 \\
\hline $7 / 19 / 93$ & $07 e$ & 0.00 & 0.00 & 0.00 & 0.00 & 0.00 & 0.00 & 0.00 & 0.00 & 0.00 & 0.00 & 0.00 & 0.00 \\
\hline $7 / 19 / 93$ & $07 f$ & 0.00 & 0.00 & 0.00 & 0.00 & 0.00 & 0.00 & 0.00 & 0.00 & 0.00 & 0.00 & 0.00 & 0.00 \\
\hline $7 / 19 / 93$ & $07 \mathrm{~g}$ & 0.00 & 0.00 & 0.00 & 0.00 & 0.00 & 0.00 & 0.00 & 0.00 & 0.00 & 0.00 & 0.00 & 0.00 \\
\hline $7 / 19 / 93$ & $07 \mathrm{~h}$ & 0.00 & 0.00 & 0.00 & 0.00 & 0.00 & 0.00 & 0.00 & 0.00 & 0.00 & 0.00 & 0.00 & 0.00 \\
\hline $7 / 20 / 93$ & $07 i$ & 0.00 & 0.00 & 0.00 & 0.00 & 0.00 & 0.00 & 0.00 & 0.00 & 0.00 & 0.00 & 0.00 & 0.00 \\
\hline $7 / 20 / 93$ & $07 j$ & 0.00 & 0.00 & 0.00 & 0.00 & 0.00 & 0.00 & 0.00 & 0.00 & 0.00 & 0.00 & 0.00 & 0.00 \\
\hline $7 / 20 / 93$ & $07 k$ & 0.00 & 0.00 & 0.00 & 0.00 & 0.00 & 0.00 & 0.00 & 0.00 & 0.00 & 0.00 & 0.00 & 0.00 \\
\hline $7 / 20 / 93$ & 071 & 0.00 & 0.00 & 0.00 & 0.00 & 0.00 & 0.00 & 0.00 & 0.00 & 0.00 & 0.00 & 0.00 & 0.00 \\
\hline $7 / 20 / 93$ & $07 m$ & 0.00 & 0.00 & 0.00 & 0.00 & 0.00 & 0.00 & 0.00 & 0.00 & 0.00 & 0.00 & 0.00 & 0.00 \\
\hline $7 / 20 / 93$ & $07 n$ & 0.00 & 0.00 & 0.00 & 0.00 & 0.00 & 0.00 & 0.00 & 0.00 & 0.00 & 0.00 & 0.00 & 0.00 \\
\hline $7 / 28 / 93$ & 070 & 0.00 & 0.00 & 0.00 & 0.00 & 0.00 & 0.00 & 0.00 & 0.00 & 0.00 & 0.00 & 0.00 & 0.00 \\
\hline $7 / 28 / 93$ & $07 p$ & 0.00 & 0.00 & 0.00 & 0.00 & 0.00 & 0.00 & 0.00 & 0.00 & 0.00 & 0.00 & 0.00 & 0.00 \\
\hline $7 / 28 / 93$ & $07 q$ & 0.00 & 0.00 & 0.00 & 0.00 & 0.00 & 0.00 & 0.00 & 0.00 & 0.00 & 0.00 & 0.00 & 0.00 \\
\hline $7 / 29 / 93$ & $07 r$ & 0.00 & 0.00 & 0.00 & 0.00 & 0.00 & 0.00 & 0.00 & 0.00 & 0.00 & 0.00 & 0.00 & 0.00 \\
\hline $7 / 29 / 93$ & $07 s$ & 0.00 & 0.00 & 0.00 & 0.00 & 0.00 & 0.00 & 0.00 & 0.00 & 0.00 & 0.00 & 0.00 & 0.00 \\
\hline $7 / 29 / 93$ & $07 t$ & 0.00 & 0.00 & 0.00 & 0.00 & 0.00 & 0.00 & 0.00 & 0.00 & 0.00 & 0.00 & 0.00 & 0.00 \\
\hline $8 / 4 / 93$ & $07 u$ & 0.00 & 0.00 & 0.00 & 0.00 & 0.00 & 0.00 & 0.00 & 0.00 & 0.00 & 0.00 & 0.00 & 0.00 \\
\hline $8 / 4 / 93$ & $07 v$ & 0.00 & 0.00 & 0.00 & 0.00 & 0.00 & 0.00 & 0.00 & 0.00 & 0.00 & 0.00 & 0.00 & 0.00 \\
\hline $8 / 5 / 93$ & $07 w$ & 0.00 & 0.00 & 0.00 & 0.00 & 0.00 & 0.00 & 0.00 & 0.00 & 0.00 & 0.00 & 0.00 & 0.00 \\
\hline
\end{tabular}




\begin{tabular}{|c|c|c|c|c|c|c|c|c|c|c|c|c|c|}
\hline DATE & EXP & AVF2 & AVF3 & AVF4 & AVF5 & AVF6 & AVF7 & S2V & S3V & S4V & S5V & $\overline{S 6 V}$ & S7V \\
\hline $8 / 5 / 93$ & $07 x$ & 0.00 & 0.00 & 0.00 & 0.00 & 0.00 & 0.00 & 0.00 & 0.00 & 0.00 & 0.00 & 0.00 & 0.00 \\
\hline $8 / 5 / 93$ & $07 y$ & 0.00 & 0.00 & 0.00 & 0.00 & 0.00 & 0.00 & 0.00 & 0.00 & 0.00 & 0.00 & 0.00 & 0.00 \\
\hline $8 / 12 / 93$ & $07 z$ & 0.00 & 0.00 & 0.00 & 0.00 & 0.00 & 0.00 & 0.00 & 0.00 & 0.00 & 0.00 & 0.00 & 0.00 \\
\hline $8 / 12 / 93$ & $08 a$ & 0.00 & 0.00 & 0.00 & 0.00 & 0.00 & 0.00 & 0.00 & 0.00 & 0.00 & 0.00 & 0.00 & 0.00 \\
\hline $8 / 12 / 93$ & $08 b$ & 0.00 & 0.00 & 0.00 & 0.00 & 0.00 & 0.00 & 0.00 & 0.00 & 0.00 & 0.00 & 0.00 & 0.00 \\
\hline $8 / 17 / 93$ & 08c & 0.55 & 0.53 & 0.53 & 0.51 & 0.54 & 0.52 & 2.01 & 2.32 & 2.40 & 2.17 & 2.22 & 2.40 \\
\hline $8 / 17 / 93$ & 08d & 0.00 & 0.00 & 0.00 & 0.00 & 0.00 & 0.00 & 0.00 & 0.00 & 0.00 & 0.00 & 0.00 & 0.00 \\
\hline $8 / 17 / 93$ & $08 \mathrm{e}$ & 0.00 & 0.00 & 0.00 & 0.00 & 0.00 & 0.00 & 0.00 & 0.00 & 0.00 & 0.00 & 0.00 & 0.00 \\
\hline $8 / 17 / 93$ & $08 f$ & 0.00 & 0.00 & 0.00 & 0.00 & 0.00 & 0.00 & 0.00 & 0.00 & 0.00 & 0.00 & 0.00 & 0.00 \\
\hline $8 / 17 / 93$ & $08 \mathrm{~g}$ & 0.00 & 0.00 & 0.00 & 0.00 & 0.00 & 0.00 & 0.00 & 0.00 & 0.00 & 0.00 & 0.00 & 0.00 \\
\hline $8 / 17 / 93$ & $08 \mathrm{~h}$ & 0.00 & 0.00 & 0.00 & 0.00 & 0.00 & 0.00 & 0.00 & 0.00 & 0.00 & 0.00 & 0.00 & 0.00 \\
\hline $8 / 17 / 93$ & $08 i$ & 0.00 & 0.00 & 0.00 & 0.00 & 0.00 & 0.00 & 0.00 & 0.00 & 0.00 & 0.00 & 0.00 & 0.00 \\
\hline $8 / 17 / 93$ & $08 j$ & 0.00 & 0.00 & 0.00 & 0.00 & 0.00 & 0.00 & 0.00 & 0.00 & 0.00 & 0.00 & 0.00 & 0.00 \\
\hline $8 / 31 / 93$ & $08 k$ & 0.56 & 0.49 & 0.49 & 0.54 & 0.56 & 0.54 & 1.54 & 1.81 & 1.83 & 1.66 & 1.58 & 1.65 \\
\hline $9 / 1 / 93$ & 081 & 0.55 & 0.48 & 0.51 & 0.48 & 0.49 & 0.53 & 1.76 & 2.35 & 2.16 & 2.22 & 2.17 & 2.00 \\
\hline 9/1/93 & $08 \mathrm{~m}$ & 0.52 & 0.48 & 0.49 & 0.52 & 0.51 & 0.47 & 1.85 & 2.15 & 2.12 & 1.94 & 1.86 & 2.00 \\
\hline $9 / 1 / 93$ & $08 n$ & 0.56 & 0.50 & 0.47 & 0.50 & 0.54 & 0.49 & 2.04 & 2.59 & 2.72 & 2.47 & 2.29 & 2.50 \\
\hline $9 / 2 / 93$ & 080 & 0.54 & 0.48 & 0.47 & 0.53 & 0.49 & 0.46 & 2.24 & 2.78 & 2.86 & 2.50 & 2.59 & 2.71 \\
\hline $9 / 2 / 93$ & $08 p$ & 0.53 & 0.49 & 0.47 & 0.50 & 0.48 & 0.44 & 2.02 & 2.29 & 2.37 & 2.19 & 2.25 & 2.35 \\
\hline $9 / 2 / 93$ & $08 q$ & 0.59 & 0.51 & 0.45 & 0.43 & 0.45 & 0.46 & 2.22 & 2.80 & 3.08 & 3.08 & 2.94 & 3.02 \\
\hline $9 / 2 / 93$ & $08 \mathrm{r}$ & 0.57 & 0.50 & 0.50 & 0.54 & 0.53 & 0.49 & 2.04 & 2.48 & 2.52 & 2.31 & 2.24 & 2.31 \\
\hline $9 / 6 / 93$ & $08 \mathrm{~s}$ & 0.55 & 0.51 & 0.42 & 0.50 & 0.54 & 0.44 & 2.49 & 2.96 & 3.31 & 3.01 & 2.84 & 3.39 \\
\hline $9 / 6 / 93$ & $08 \mathrm{t}$ & 0.51 & 0.47 & 0.46 & 0.48 & 0.49 & 0.52 & 2.43 & 2.81 & 2.77 & 2.67 & 2.57 & 2.66 \\
\hline $9 / 6 / 93$ & $08 u$ & 0.50 & 0.48 & 0.48 & 0.51 & 0.57 & 0.55 & 2.93 & 3.42 & 3.33 & 3.21 & 2.93 & 3.06 \\
\hline $9 / 6 / 93$ & $08 \mathrm{v}$ & 0.51 & 0.48 & 0.49 & 0.47 & 0.55 & 0.49 & 2.54 & 2.84 & 2.75 & 2.82 & 2.34 & 2.79 \\
\hline $9 / 6 / 93$ & $08 w$ & 0.53 & 0.48 & 0.46 & 0.56 & 0.46 & 0.48 & 2.72 & 3.15 & 3.24 & 2.78 & 3.27 & 3.18 \\
\hline $9 / 6 / 93$ & $08 x$ & 0.48 & 0.48 & 0.45 & 0.49 & 0.44 & 0.44 & 2.51 & 2.62 & 2.79 & 2.60 & 2.87 & 2.89 \\
\hline $9 / 7 / 93$ & $08 y$ & 0.55 & 0.45 & 0.46 & 0.48 & 0.53 & 0.46 & 2.87 & 3.48 & 3.61 & 3.30 & 3.10 & 3.19 \\
\hline $9 / 7 / 93$ & $08 z$ & 0.48 & 0.46 & 0.43 & 0.52 & 0.52 & 0.44 & 2.81 & 3.05 & 3.20 & 2.59 & 2.61 & 3.07 \\
\hline $9 / 7 / 93$ & $09 a$ & 0.52 & 0.44 & 0.45 & 0.45 & 0.45 & 0.44 & 3.15 & 3.65 & 3.51 & 3.53 & 3.28 & 3.33 \\
\hline $9 / 7 / 93$ & $09 b$ & 0.48 & 0.44 & 0.43 & 0.45 & 0.48 & 0.46 & 2.95 & 3.04 & 3.22 & 3.13 & 2.92 & 3.12 \\
\hline $9 / 7 / 93$ & $09 c$ & 0.58 & 0.52 & 0.47 & 0.51 & 0.54 & 0.53 & 1.85 & 2.21 & 2.36 & 2.15 & 2.05 & 2.16 \\
\hline $9 / 7 / 93$ & $09 d$ & 0.54 & 0.51 & 0.48 & 0.50 & 0.54 & 0.47 & 1.94 & 2.13 & 2.29 & 2.00 & 1.93 & 2.23 \\
\hline $9 / 8 / 93$ & $09 \mathrm{e}$ & 0.55 & 0.46 & 0.51 & 0.55 & 0.54 & 0.49 & 1.88 & 2.42 & 2.25 & 2.05 & 2.05 & 2.28 \\
\hline $9 / 8 / 93$ & $09 f$ & 0.55 & 0.56 & 0.59 & 0.52 & 0.54 & 0.54 & 1.69 & 1.73 & 1.69 & 1.80 & 1.70 & 1.82 \\
\hline $9 / 13 / 93$ & $09 g$ & 0.51 & 0.45 & 0.46 & 0.43 & 0.43 & 0.44 & 3.23 & 3.66 & 3.44 & 3.44 & 3.36 & 3.40 \\
\hline $9 / 13 / 93$ & $09 \mathrm{~g}$ & 0.51 & 0.45 & 0.46 & 0.43 & 0.43 & 0.44 & 3.03 & 3.42 & 3.22 & 3.22 & 3.15 & 3.18 \\
\hline $9 / 13 / 93$ & $09 \mathrm{~h}$ & 0.46 & 0.42 & 0.39 & 0.44 & 0.44 & 0.43 & 2.97 & 2.98 & 3.31 & 2.58 & 2.59 & 3.15 \\
\hline $9 / 14 / 93$ & $09 i$ & 0.46 & 0.47 & 0.45 & 0.43 & 0.44 & 0.44 & 3.52 & 3.38 & 3.52 & 3.65 & 3.63 & 3.56 \\
\hline $9 / 14 / 93$ & 091 & 0.47 & 0.43 & 0.42 & 0.42 & 0.43 & 0.43 & 3.51 & 3.60 & 3.68 & 3.64 & 3.57 & 3.49 \\
\hline $9 / 14 / 93$ & $09 j$ & 0.43 & 0.41 & 0.44 & 0.43 & 0.45 & 0.44 & 3.33 & 3.28 & 3.16 & 3.20 & 3.18 & 3.17 \\
\hline $9 / 14 / 93$ & 09k & $\overline{0.47}$ & 0.44 & 0.42 & 0.43 & 0.44 & 0.45 & 3.54 & 3.78 & 3.82 & 3.76 & 3.75 & 3.66 \\
\hline $9 / 14 / 93$ & 091 & 0.41 & 0.45 & 0.42 & 0.41 & 0.41 & 0.39 & 3.31 & 3.14 & 3.25 & 3.29 & 3.33 & 3.31 \\
\hline $9 / 14 / 93$ & 09m & 0.45 & 0.43 & 0.44 & 0.43 & 0.42 & 0.40 & 3.81 & 3.87 & 3.83 & 3.89 & 4.09 & 4.14 \\
\hline $9 / 15 / 93$ & $09 n$ & 0.42 & 0.38 & 0.36 & 0.35 & 0.33 & 0.32 & 3.29 & 3.46 & 3.65 & 3.65 & 3.80 & 3.85 \\
\hline $9 / 17 / 93$ & 090 & 0.46 & 0.39 & 0.38 & 0.39 & 0.38 & 0.37 & 3.56 & 3.92 & 3.94 & 3.82 & 3.94 & 4.02 \\
\hline $9 / 17 / 93$ & $09 p$ & 0.44 & 0.42 & 0.38 & 0.39 & 0.38 & 0.36 & 3.74 & 3.72 & 3.91 & 3.90 & 3.97 & 4.17 \\
\hline $9 / 17 / 93$ & $09 q$ & 0.44 & 0.40 & 0.39 & 0.38 & 0.38 & 0.37 & 3.81 & 3.88 & 3.89 & 3.93 & 3.93 & 4.07 \\
\hline $9 / 20 / 93$ & $09 r$ & 0.47 & 0.42 & 0.40 & 0.42 & 0.44 & 0.38 & 3.59 & 3.88 & 3.92 & 3.92 & 3.71 & 3.95 \\
\hline $9 / 20 / 93$ & $09 \mathrm{~s}$ & 0.44 & 0.44 & 0.00 & 0.00 & 0.00 & 0.00 & 3.75 & 3.74 & 0.00 & 0.00 & 0.00 & 0.00 \\
\hline
\end{tabular}




\begin{tabular}{|c|c|c|c|c|c|c|c|c|c|c|c|c|c|}
\hline DATE & EXP & AVF2 & AVF3 & AVF4 & AVF5 & AVF6 & AVF7 & $\mathrm{S} 2 \mathrm{~V}$ & S3V & S4V & S5V & S6V & S7V \\
\hline $9 / 20 / 93$ & $09 t$ & 0.44 & 0.41 & 0.43 & 0.40 & 0.41 & 0.40 & 3.67 & 3.77 & 3.79 & 3.73 & 3.83 & 3.98 \\
\hline $9 / 20 / 93$ & $09 u$ & 0.44 & 0.46 & 0.44 & 0.00 & 0.46 & 0.42 & 3.94 & 3.72 & 3.92 & 0.00 & 3.77 & 4.03 \\
\hline $9 / 21 / 93$ & $09 v$ & 0.46 & 0.44 & 0.40 & 0.41 & 0.41 & 0.37 & 3.58 & 3.73 & 4.05 & 4.07 & 3.90 & 4.16 \\
\hline $9 / 21 / 93$ & $09 w$ & 0.43 & 0.40 & 0.40 & 0.40 & 0.44 & 0.41 & 3.82 & 3.94 & 4.09 & 3.95 & 3.63 & 4.09 \\
\hline $9 / 21 / 93$ & $09 x$ & 0.45 & 0.45 & 0.39 & 0.40 & 0.48 & 0.38 & 3.73 & 3.69 & 4.15 & 4.05 & 3.50 & 4.11 \\
\hline $9 / 22 / 93$ & $\overline{09 y}$ & 0.00 & 0.00 & 0.00 & 0.00 & 0.00 & 0.00 & 0.00 & 0.00 & 0.00 & 0.00 & 0.00 & 0.00 \\
\hline $9 / 22 / 93$ & $10 a$ & 0.00 & 0.00 & 0.00 & 0.00 & 0.00 & 0.00 & 0.00 & 0.00 & 0.00 & 0.00 & 0.00 & 0.00 \\
\hline $9 / 22 / 93$ & $10 b$ & 0.00 & 0.00 & 0.00 & 0.00 & 0.00 & 0.00 & 0.00 & 0.00 & 0.00 & 0.00 & 0.00 & 0.00 \\
\hline $9 / 22 / 93$ & $10 c$ & 0.00 & 0.00 & 0.00 & 0.00 & 0.00 & 0.00 & 0.00 & 0.00 & 0.00 & 0.00 & 0.00 & 0.00 \\
\hline $9 / 23 / 93$ & $10 d$ & 0.00 & $\overline{0.00}$ & 0.00 & 0.00 & 0.00 & 0.00 & 0.00 & 0.00 & 0.00 & 0.00 & 0.00 & 0.00 \\
\hline $9 / 23 / 93$ & $10 \mathrm{e}$ & 0.00 & 0.00 & 0.00 & 0.00 & 0.00 & 0.00 & 0.00 & 0.00 & 0.00 & 0.00 & 0.00 & 0.00 \\
\hline $9 / 23 / 93$ & $10 f$ & 0.00 & 0.00 & 0.00 & 0.00 & 0.00 & 0.00 & 0.00 & 0.00 & 0.00 & 0.00 & 0.00 & 0.00 \\
\hline $9 / 23 / 93$ & $10 \mathrm{~g}$ & 0.00 & 0.00 & 0.00 & 0.00 & 0.00 & 0.00 & 0.00 & 0.00 & 0.00 & 0.00 & 0.00 & 0.00 \\
\hline $9 / 23 / 93$ & $10 \mathrm{~h}$ & 0.00 & 0.00 & 0.00 & 0.00 & 0.00 & 0.00 & 0.00 & 0.00 & 0.00 & 0.00 & 0.00 & 0.00 \\
\hline $9 / 23 / 93$ & $10 i$ & 0.00 & 0.00 & 0.00 & 0.00 & 0.00 & 0.00 & 0.00 & 0.00 & 0.00 & 0.00 & 0.00 & 0.00 \\
\hline $9 / 24 / 93$ & 10j & 0.00 & 0.00 & 0.00 & 0.00 & 0.00 & 0.00 & 0.00 & 0.00 & 0.00 & 0.00 & 0.00 & 0.00 \\
\hline $9 / 24 / 93$ & $10 \mathrm{~K}$ & 0.00 & 0.00 & 0.00 & 0.00 & 0.00 & 0.00 & 0.00 & 0.00 & 0.00 & 0.00 & 0.00 & 0.00 \\
\hline $9 / 24 / 93$ & 101 & 0.00 & 0.00 & 0.00 & 0.00 & 0.00 & 0.00 & 0.00 & 0.00 & 0.00 & 0.00 & 0.00 & 0.00 \\
\hline $9 / 24 / 93$ & $10 \mathrm{~m}$ & 0.00 & 0.00 & 0.00 & 0.00 & 0.00 & 0.00 & 0.00 & 0.00 & 0.00 & 0.00 & 0.00 & 0.00 \\
\hline $10 / 6 / 93$ & $10 n$ & 0.44 & 0.40 & 0.39 & 0.40 & 0.44 & 0.38 & 3.64 & 3.74 & 3.90 & 3.77 & 3.41 & 3.97 \\
\hline $10 / 6 / 93$ & 100 & 0.40 & 0.41 & 0.38 & 0.39 & 0.44 & 0.41 & 3.79 & 3.66 & 3.92 & 3.84 & 3.45 & 3.87 \\
\hline $10 / 7 / 93$ & $10 p$ & 0.44 & 0.42 & 0.38 & 0.38 & 0.40 & 0.39 & 3.59 & 3.65 & 3.91 & 3.93 & 3.71 & 3.83 \\
\hline $10 / 11 / 93$ & $10 q$ & 0.43 & 0.43 & 0.40 & 0.41 & 0.40 & 0.38 & 3.65 & 3.66 & 3.79 & 3.66 & 3.72 & 3.91 \\
\hline $10 / 11 / 93$ & $10 r$ & 0.46 & 0.45 & 0.44 & 0.45 & 0.50 & 0.43 & 3.62 & 3.64 & 3.67 & 3.51 & 3.39 & 3.62 \\
\hline $10 / 12 / 93$ & $10 \mathrm{~s}$ & 0.48 & 0.41 & 0.42 & 0.41 & 0.44 & 0.43 & 3.71 & 3.87 & 3.97 & 3.87 & 3.69 & 3.81 \\
\hline $10 / 12 / 93$ & $10 t$ & 0.46 & 0.43 & 0.43 & 0.40 & 0.50 & 0.42 & 3.60 & 3.83 & 3.72 & 3.57 & 3.32 & 3.73 \\
\hline $10 / 12 / 93$ & $10 u$ & 0.45 & 0.38 & 0.39 & 0.48 & 0.53 & 0.43 & 3.49 & 3.87 & 3.80 & 3.07 & 2.77 & 3.48 \\
\hline $10 / 13 / 93$ & $10 w$ & 0.44 & 0.41 & 0.38 & 0.40 & 0.48 & 0.42 & 3.66 & 3.64 & 3.86 & 3.66 & 3.29 & 3.62 \\
\hline $10 / 14 / 93$ & $10 y$ & 0.38 & 0.38 & 0.39 & 0.36 & 0.38 & 0.43 & 4.21 & 3.85 & 3.97 & 4.23 & 3.92 & 3.50 \\
\hline $10 / 18 / 93$ & $10 z$ & 0.50 & 0.44 & 0.42 & 0.48 & 0.48 & 0.48 & 2.58 & 3.12 & 3.27 & 2.85 & 2.91 & 2.90 \\
\hline $10 / 18 / 93$ & $11 a$ & 0.56 & 0.52 & 0.47 & 0.50 & 0.44 & 0.46 & 2.51 & 2.93 & 3.14 & 2.85 & 3.34 & 3.14 \\
\hline $10 / 18 / 93$ & $11 b$ & 0.56 & 0.47 & 0.46 & 0.55 & 0.50 & 0.49 & 2.49 & 3.05 & 3.10 & 2.69 & 2.81 & 2.93 \\
\hline $10 / 19 / 93$ & $11 c$ & 0.51 & 0.48 & 0.46 & 0.49 & 0.53 & 0.51 & 2.67 & 3.00 & 3.27 & 2.91 & 2.79 & 2.88 \\
\hline $10 / 19 / 93$ & $11 d$ & 0.52 & 0.45 & 0.43 & 0.52 & 0.58 & 0.51 & 2.68 & 3.20 & 3.46 & 2.85 & 2.58 & 2.99 \\
\hline $10 / 19 / 93$ & $11 \mathrm{e}$ & 0.55 & 0.48 & 0.47 & 0.50 & 0.48 & 0.48 & 2.60 & 3.03 & 3.12 & 3.09 & 3.20 & 3.10 \\
\hline $10 / 19 / 93$ & $11 f$ & 0.53 & 0.47 & 0.47 & 0.50 & 0.49 & 0.50 & 2.74 & 3.19 & 3.23 & 2.91 & 3.12 & 3.15 \\
\hline $10 / 20 / 93$ & $11 \mathrm{~g}$ & 0.52 & 0.44 & 0.45 & 0.47 & 0.56 & 0.56 & 2.69 & 3.32 & 3.35 & 3.11 & 2.74 & 2.70 \\
\hline $10 / 20 / 93$ & $11 \mathrm{~h}$ & 0.52 & 0.46 & 0.47 & 0.53 & 0.54 & 0.49 & 2.65 & 3.05 & 3.08 & 2.73 & 2.72 & 3.05 \\
\hline $10 / 20 / 93$ & $11 j$ & 0.52 & 0.47 & 0.45 & 0.56 & 0.63 & 0.53 & 2.66 & 3.14 & 3.25 & 2.52 & 2.34 & 2.74 \\
\hline $10 / 21 / 93$ & $11 \mathrm{j}$ & 0.51 & 0.48 & 0.47 & 0.52 & 0.48 & 0.49 & 2.76 & 3.02 & 3.10 & 2.84 & 3.08 & 2.99 \\
\hline $10 / 21 / 93$ & $11 k$ & 0.50 & 0.43 & 0.44 & 0.50 & 0.51 & 0.47 & 2.73 & 3.25 & 3.20 & 2.77 & 2.84 & 3.05 \\
\hline $10 / 21 / 93$ & 111 & 0.52 & 0.45 & 0.46 & 0.50 & 0.49 & 0.49 & 2.66 & 3.24 & 3.13 & 2.87 & 3.02 & 3.03 \\
\hline $10 / 21 / 93$ & $11 \mathrm{~m}$ & 0.51 & 0.49 & 0.44 & 0.52 & 0.54 & 0.48 & 2.80 & 3.12 & 3.46 & 2.75 & 2.79 & 3.15 \\
\hline $10 / 21 / 93$ & $11 n$ & 0.51 & 0.48 & 0.46 & 0.58 & 0.58 & 0.49 & 2.67 & 3.07 & 3.08 & 2.31 & 2.36 & 2.93 \\
\hline $10 / 22 / 93$ & 110 & 0.51 & 0.44 & 0.46 & 0.53 & 0.53 & 0.48 & 2.75 & 3.31 & 3.26 & 2.76 & 2.80 & 3.06 \\
\hline $10 / 22 / 93$ & $11 a$ & 0.51 & 0.46 & 0.47 & 0.48 & 0.55 & 0.48 & 2.78 & 3.21 & 3.20 & 3.01 & 2.92 & 3.01 \\
\hline $10 / 22 / 93$ & $11 r$ & 0.49 & 0.48 & 0.46 & 0.53 & 0.63 & 0.49 & 2.79 & 3.05 & 3.13 & 2.55 & 2.31 & 2.95 \\
\hline $10 / 22 / 93$ & $11 \mathrm{~s}$ & 0.49 & 0.46 & 0.47 & 0.54 & 0.61 & 0.50 & 2.75 & 3.21 & 2.99 & 2.35 & 2.12 & 2.79 \\
\hline $10 / 22 / 93$ & $11 \mathrm{t}$ & 0.51 & 0.49 & 0.46 & 0.50 & 0.50 & 0.47 & 2.81 & 3.14 & 3.24 & 2.99 & 3.05 & 3.11 \\
\hline $10 / 25 / 93$ & $11 \mathrm{u}$ & 0.52 & 0.46 & 0.44 & 0.53 & 0.53 & 0.45 & 2.70 & 3.15 & 3.19 & 2.77 & 2.75 & 3.27 \\
\hline
\end{tabular}




\begin{tabular}{|c|c|c|c|c|c|c|c|c|c|c|c|c|c|}
\hline$\overline{\text { DATE }}$ & $\overline{X P}$ & AVF2 & AVF3 & $\widehat{\text { AVF4 }}$ & $\widehat{\text { AVF5 }}$ & AVF6 & AVF7 & $\mathrm{S} 2 \mathrm{~V}$ & S3V & S4V & S5V & S6V & STV \\
\hline $10 / 25 / 93$ & $11 v$ & 0.54 & 0.46 & 0.48 & 0.53 & 0.54 & 0.53 & 2.69 & 3.30 & 3.13 & 2.98 & 2.88 & 2.92 \\
\hline $10 / 25 / 93$ & $11 w$ & 55 & 0.47 & 0.43 & 0.52 & 0.59 & 0.50 & 2.72 & 3.22 & 3.32 & 2.74 & 2.55 & 3.03 \\
\hline $10 / 25 / 93$ & $11 x$ & .50 & 0.45 & 0.49 & 0.69 & 0.59 & 0.53 & 2.93 & 3.40 & 3.18 & 2.13 & 2.47 & 2.89 \\
\hline $11 / 14 / 93$ & $10 x$ & .43 & 0.42 & 0.36 & 0.38 & 0.42 & 0.39 & 3.64 & 3.67 & 4.12 & 3.95 & 3.67 & 3.84 \\
\hline $11 / 21 / 93$ & 110 & .48 & 0.41 & 0.48 & 0.43 & 0.44 & 0.52 & 2.89 & 3.35 & 3.12 & 3.38 & 3.15 & 2.77 \\
\hline $2 / 23 / 94$ & & 0.00 & 0.00 & 0.00 & 0.00 & 0.00 & 0.00 & & & & & & \\
\hline $2 / 23 / 94$ & & .00 & 0.00 & 0.00 & 0.00 & 0.00 & .00 & & & & & & \\
\hline $2 / 23 / 94$ & & .00 & 0.00 & 0.00 & 0.00 & 0.00 & .00 & & & & & & \\
\hline $2 / 24 / 94$ & & .00 & 0.00 & 0.00 & 0.00 & 0.00 & .00 & & & & & & \\
\hline $4 / 18 / 94$ & $12 a$ & 00 & 0.00 & 0.00 & 0.00 & 0.00 & 0.00 & 0.00 & 0.00 & 0.00 & 0.00 & 0.00 & 0.00 \\
\hline $4 / 18 / 94$ & $12 b$ & 0.00 & 0.00 & 0.00 & 0.00 & 0.00 & 0.00 & 0.00 & 0.00 & 0.00 & 0.00 & 0.00 & 0.00 \\
\hline $4 / 18 / 94$ & $12 c$ & 0.00 & 0.00 & 0.00 & 0.00 & 0.00 & 0.00 & 0.00 & 0.00 & 0.00 & 0.00 & 0.00 & 0.00 \\
\hline $4 / 18 / 94$ & $12 d$ & 0.00 & 0.00 & 0.00 & 0.00 & 0.00 & 0.00 & 0.00 & 0.00 & 0.00 & 0.00 & 0.00 & 0.00 \\
\hline $4 / 18 / 94$ & $12 e$ & 0.00 & 0.00 & 0.00 & 0.00 & 0.00 & 0.00 & 0.00 & .00 & .00 & 0.00 & 0.00 & 0.00 \\
\hline $4 / 18 / 94$ & A12 & 0.00 & 0.00 & 0.00 & 0.00 & 0.00 & 0.00 & & & & & & \\
\hline $4 / 21 / 94$ & $\mathrm{~B} 12$ & 0.00 & 0.00 & 0.00 & 0.00 & 0.00 & .00 & & & & & & \\
\hline $5 / 2 / 94$ & $12 \mathrm{f}$ & 0.00 & 0.00 & 0.00 & 0.00 & 0.00 & .00 & 0.00 & 0.00 & 0.00 & 0.00 & 0.00 & 0.00 \\
\hline $5 / 2 / 94$ & $12 \mathrm{~g}$ & 0.00 & 0.00 & 0.00 & 0.00 & 0.00 & .00 & 0.00 & 0.00 & 0.00 & 0.00 & 0.00 & 0.00 \\
\hline $5 / 2 / 94$ & $12 \mathrm{~h}$ & 0.00 & 0.00 & 0.00 & 0.00 & 0.00 & .00 & 0.00 & 0.00 & 0.00 & 0.00 & 0.00 & .00 \\
\hline $5 / 2 / 94$ & $12 i$ & 0.00 & 0.00 & 0.00 & 0.00 & 0.00 & & 0.00 & 0.00 & 0.00 & 0.00 & 0.00 & .00 \\
\hline $5 / 2 / 94$ & $12 \mathrm{j}$ & 0.73 & 0.71 & 0.59 & 0.62 & 0.63 & & 0.15 & 0.1 & 0.20 & 0.19 & 0.19 & 0.24 \\
\hline $5 / 3 / 94$ & $12 r$ & 0.59 & 0.61 & 0.57 & 0.52 & 0.52 & .50 & 0.09 & 0.08 & 0.09 & 0.09 & 0.10 & $\overline{0.12}$ \\
\hline $5 / 3 / 94$ & $12 \mathrm{k}$ & 0.00 & 0.00 & 0.00 & 0.00 & 0.00 & 0.00 & 0.00 & 0.00 & 0.00 & 0.00 & 0.00 & 0.00 \\
\hline $5 / 3 / 94$ & 121 & 0.00 & 0.00 & 0.00 & 0.00 & 0.00 & 0.00 & 0.00 & 0.00 & 0.00 & 0.00 & 0.00 & 0.00 \\
\hline $5 / 3 / 94$ & $12 \mathrm{~m}$ & 0.00 & 0.00 & 0.00 & 0.00 & 0.00 & 0.00 & 0.00 & 0.00 & 0.00 & 0.00 & 0.00 & 0.00 \\
\hline $5 / 3 / 94$ & $12 n$ & 0.00 & 0.00 & 0.00 & 0.00 & 0.00 & .00 & 0.00 & 0.00 & 0.00 & 0.00 & 0.00 & 0.00 \\
\hline $5 / 3 / 94$ & 120 & 0.59 & 0.57 & 0.57 & 0.53 & 0.53 & 0.49 & 0.15 & .16 & 0.17 & 0.17 & .18 & 0.21 \\
\hline $5 / 3 / 94$ & $12 p$ & 0.00 & 0.00 & 0.00 & 0.00 & 0.00 & 0.00 & 0.00 & 1.00 & 0.00 & 0.00 & 0.00 & 0.00 \\
\hline $5 / 3 / 94$ & $12 q$ & 0.00 & 0.00 & 0.00 & 0.00 & 0.00 & 0.00 & 0.00 & 0.00 & 0.00 & 0.00 & 0.00 & 0.00 \\
\hline $5 / 3 / 94$ & $12 \mathrm{~s}$ & 0.56 & 0.58 & 0.59 & 0.57 & 0.53 & 0.46 & 0.38 & 0.39 & 0.40 & 0.41 & 0.43 & 0.51 \\
\hline $5 / 3 / 94$ & $12 t$ & 0.62 & 0.63 & 0.60 & 0.61 & 0.61 & 0.55 & 0.40 & 0.41 & 0.45 & 0.43 & 0.44 & 0.49 \\
\hline $5 / 5 / 94$ & $12 u$ & 0.00 & 0.00 & 0.00 & 0.00 & 0.00 & 0 & 0.00 & 0.00 & 0.00 & 0.00 & 0.00 & 0.00 \\
\hline $5 / 5 / 94$ & $12 v$ & 0.58 & 0.55 & 0.56 & 0.53 & 0.54 & & 0.10 & $\overline{0.11}$ & 0.11 & 0.11 & 0.11 & 0.13 \\
\hline $5 / 6 / 94$ & $12 w$ & 0.54 & 0.51 & 0.51 & 0.51 & 0.53 & 0.50 & 1.00 & 1.09 & 1.13 & 1.12 & 1.08 & 1.09 \\
\hline $5 / 6 / 94$ & $12 x$ & 0.54 & 0.55 & 0.59 & 0.59 & .58 & & 1.30 & 1.33 & 1.36 & 1.38 & 1.39 & 1.44 \\
\hline $5 / 6 / 94$ & $12 y$ & 0.56 & 0.55 & 0.55 & 0.54 & 0.54 & 0.55 & 1.45 & 1.79 & 1.99 & 2.10 & 2.17 & 2.24 \\
\hline $5 / 6 / 94$ & $12 z$ & 0.50 & 0.46 & 0.49 & $\overline{0.47}$ & 0.46 & 0.42 & 0.75 & 0.76 & 0.75 & $\overline{0.74}$ & 0.76 & 0.83 \\
\hline $5 / 6 / 94$ & $\overline{13 a}$ & 0.57 & 0.53 & 0.53 & 0.52 & 0.53 & .53 & 1.20 & 1.23 & 1.23 & 1.25 & 1.21 & 1.25 \\
\hline $5 / 6 / 94$ & $13 b$ & 0.53 & 0.52 & 0.51 & 0.50 & .54 & .51 & 1.58 & 3 & 1.81 & 1.79 & 1.72 & 1.83 \\
\hline $5 / 6 / 94$ & $13 c$ & 0.58 & 0.56 & 0.56 & 0.55 & 0.54 & & 1.79 & $\overline{2.3}$ & 2.65 & 2.69 & 2.77 & 2.81 \\
\hline $5 / 9 / 94$ & $13 d$ & 0.00 & 0.00 & 0.00 & 0.00 & 0.00 & & 0.00 & 0.0 & 0.00 & 0.00 & 0.00 & 0.00 \\
\hline $5 / 9 / 94$ & $13 e$ & 0.45 & 0.44 & $0 . \overline{42}$ & 0.49 & 0.50 & & 1.00 & 1.12 & 1.20 & 1.05 & 1.05 & 1.08 \\
\hline $5 / 9 / 94$ & $13 f$ & 0.49 & 0.47 & 0.46 & 0.44 & 0.46 & 0.46 & 1.87 & 2.01 & 2.09 & 2.11 & 2.12 & 2.10 \\
\hline $5 / 9 / 94$ & $13 \mathrm{~g}$ & 0.56 & 0.51 & 0.50 & 0.50 & 0.50 & 50 & 2.13 & 2.41 & 2.59 & 2.60 & 2.63 & 2.65 \\
\hline $5 / 9 / 94$ & $13 \mathrm{~h}$ & 0.60 & 0.57 & 0.51 & 0.55 & 0.52 & & 2.64 & 3.32 & 3.80 & 3.75 & 4.00 & 4.09 \\
\hline $5 / 11 / 94$ & $13 i$ & 0.00 & 0.00 & 0.00 & 0.00 & 0.00 & 0.00 & 0.00 & 0.00 & 0.00 & 0.00 & 0.00 & 0.00 \\
\hline $5 / 11 / 94$ & $13 j$ & 0.48 & 0.41 & 0.41 & 0.40 & 0.50 & 0.47 & 1.76 & 1.89 & 2.05 & 2.13 & 1.76 & 1.86 \\
\hline $5 / 11 / 94$ & & & 0.40 & 0.44 & 0.44 & 0.47 & & 2.36 & 2.59 & 2.48 & 2.54 & 2.62 & 2.39 \\
\hline $5 / 11 / 94$ & 131 & 0.48 & 0.44 & 0.40 & 0.40 & 0.41 & 0.46 & 2.85 & 3.18 & 3.60 & 3.50 & 3.38 & 3.16 \\
\hline $5 / 11 / 94$ & $13 \mathrm{~m}$ & 0.54 & 0.52 & 0.50 & 0.49 & 0.48 & 0.47 & 4.38 & 4.75 & 5.36 & 5.44 & 5.42 & 5.57 \\
\hline
\end{tabular}




\begin{tabular}{|c|c|c|c|c|c|c|c|c|c|c|c|c|c|}
\hline DATE & EXP & AVF2 & AVF3 & AVF4 & AVF5 & $\overline{\text { AVF6 }}$ & AVF7 & S2V & S3V & S4V & S5V & S6V & S7V \\
\hline $5 / 11 / 94$ & $13 n$ & 0.43 & 0.46 & 0.52 & 0.47 & 0.40 & 0.37 & 0.73 & 0.71 & 0.55 & 0.64 & 0.65 & 0.77 \\
\hline $5 / 11 / 94$ & 130 & 0.37 & 0.39 & 0.36 & 0.39 & 0.36 & 0.38 & 1.80 & 1.77 & 1.87 & 1.71 & 1.93 & 1.94 \\
\hline $5 / 11 / 94$ & $13 p$ & 0.43 & 0.47 & 0.41 & 0.47 & 0.42 & 0.50 & 2.59 & 2.56 & 2.95 & 2.58 & 2.82 & 2.28 \\
\hline $5 / 11 / 94$ & $13 q$ & 0.51 & 0.45 & 0.41 & 0.42 & 0.44 & 0.44 & 2.85 & 3.36 & 3.61 & 3.44 & 3.34 & 3.33 \\
\hline $5 / 11 / 94$ & $13 r$ & 0.55 & 0.53 & 0.49 & 0.48 & 0.46 & 0.47 & 4.31 & 4.82 & 5.23 & 5.29 & 5.37 & 5.46 \\
\hline $5 / 13 / 94$ & $13 \mathrm{~s}$ & 0.51 & 0.49 & 0.46 & 0.47 & 0.45 & 0.45 & 5.08 & 5.34 & 5.62 & 5.33 & 5.39 & 5.39 \\
\hline $5 / 13 / 94$ & $13 t$ & 0.46 & 0.44 & 0.41 & 0.42 & 0.46 & 0.46 & 3.61 & 3.59 & 3.96 & 3.81 & 3.38 & 3.45 \\
\hline $5 / 13 / 94$ & $13 u$ & 0.40 & 0.42 & 0.40 & 0.42 & 0.42 & 0.42 & 3.01 & 2.86 & 3.01 & 2.88 & 2.91 & 2.97 \\
\hline $5 / 13 / 94$ & $13 v$ & 0.36 & 0.36 & 0.38 & 0.37 & 0.35 & 0.38 & 2.05 & 2.05 & 1.96 & 2.03 & 2.09 & 2.05 \\
\hline $5 / 13 / 94$ & $13 w$ & 0.34 & 0.35 & 0.31 & 0.30 & 0.27 & 0.09 & 0.74 & 0.72 & 0.82 & 0.79 & 0.82 & 0.83 \\
\hline $5 / 13 / 94$ & $13 x$ & 0.43 & $\overline{0.41}$ & 0.39 & 0.40 & 0.42 & 0.40 & 4.94 & 5.15 & 5.31 & 5.21 & 4.94 & 5.11 \\
\hline $5 / 13 / 94$ & $13 y$ & 0.46 & 0.45 & 0.41 & 0.43 & 0.45 & 0.40 & 3.63 & 3.68 & 3.87 & 3.65 & 3.46 & 4.01 \\
\hline $5 / 13 / 94$ & $13 z$ & 0.41 & 0.44 & 0.39 & 0.41 & 0.45 & 0.42 & 2.83 & 2.66 & 3.01 & 2.92 & 2.60 & 2.81 \\
\hline $5 / 16 / 94$ & $14 a$ & 0.35 & 0.37 & 0.34 & 0.35 & 0.37 & 0.37 & 1.85 & 1.82 & 1.96 & 1.95 & 1.84 & 1.85 \\
\hline $5 / 16 / 94$ & $14 b$ & 0.34 & 0.32 & 0.24 & 0.16 & 0.02 & 0.01 & 0.59 & 0.57 & 0.70 & 0.89 & 2.65 & 3.93 \\
\hline $5 / 16 / 94$ & $14 c$ & 0.43 & 0.41 & 0.40 & 0.39 & 0.41 & 0.41 & 5.18 & 5.21 & 5.37 & 5.24 & 4.96 & 4.93 \\
\hline $5 / 16 / 94$ & $14 d$ & 0.46 & 0.42 & 0.40 & 0.42 & 0.40 & 0.41 & 3.50 & 3.63 & 3.70 & 3.52 & 3.60 & 3.65 \\
\hline $5 / 16 / 94$ & $14 \mathrm{e}$ & 0.33 & 0.33 & 0.30 & 0.31 & 0.34 & 0.34 & 2.78 & 2.65 & 2.85 & 2.74 & 2.74 & 2.77 \\
\hline $5 / 16 / 94$ & $14 f$ & 0.35 & 0.33 & 0.31 & 0.32 & 0.32 & 0.33 & 1.93 & 1.95 & 2.11 & 2.04 & 2.07 & 2.04 \\
\hline $5 / 17 / 94$ & 149 & 0.12 & 0.01 & 0.00 & 0.00 & 0.00 & 0.02 & 0.81 & 3.58 & 3.75 & 4.48 & 4.53 & 0.47 \\
\hline $5 / 17 / 94$ & $14 \mathrm{~h}$ & 0.41 & 0.40 & 0.36 & 0.38 & 0.38 & 0.38 & 5.09 & 4.95 & 5.42 & 5.08 & 4.99 & 5.04 \\
\hline $5 / 17 / 94$ & $14 i$ & 0.41 & 0.38 & 0.34 & 0.34 & 0.32 & 0.31 & 3.97 & 4.00 & 4.40 & 4.32 & 4.47 & 4.62 \\
\hline $5 / 17 / 94$ & $14 \mathrm{j}$ & 0.28 & 0.27 & 0.23 & 0.22 & 0.19 & 0.16 & 3.27 & 3.35 & 4.02 & 4.24 & 4.82 & 5.49 \\
\hline $5 / 17 / 94$ & $14 k$ & 0.28 & 0.18 & 0.09 & 0.06 & 0.04 & 0.04 & 2.49 & 3.19 & 4.55 & 5.50 & 6.07 & 6.45 \\
\hline $5 / 17 / 94$ & $14 \mid$ & 0.10 & 0.02 & 0.01 & 0.01 & 0.00 & 0.00 & 1.81 & 3.26 & 4.19 & 4.58 & 4.73 & 4.82 \\
\hline $5 / 17 / 94$ & $14 \mathrm{~m}$ & 0.41 & 0.43 & 0.39 & 0.39 & 0.39 & 0.39 & 5.11 & 4.65 & 5.16 & 5.06 & 5.15 & 5.05 \\
\hline $5 / 20 / 94$ & $14 n$ & 0.42 & 0.40 & 0.38 & 0.36 & 0.37 & 0.41 & 3.62 & 3.64 & 3.92 & 4.12 & 4.02 & 3.71 \\
\hline $5 / 20 / 94$ & 140 & 0.32 & 0.32 & 0.36 & $\overline{0.36}$ & 0.38 & 0.40 & 2.97 & 2.83 & 3.02 & 3.01 & 2.90 & 2.79 \\
\hline $5 / 20 / 94$ & $14 p$ & 0.34 & 0.34 & 0.33 & 0.31 & 0.31 & 0.35 & 1.97 & 1.97 & 2.11 & 2.14 & 2.15 & 2.06 \\
\hline $5 / 20 / 94$ & $14 q$ & 0.35 & 0.39 & 0.35 & 0.29 & 0.39 & 0.33 & 0.68 & 0.59 & 0.64 & 0.76 & 0.56 & 0.67 \\
\hline $5 / 20 / 94$ & $14 r$ & 0.47 & 0.44 & 0.41 & 0.46 & 0.46 & 0.44 & 5.36 & 5.32 & 5.72 & 5.26 & 5.07 & 5.18 \\
\hline $5 / 20 / 94$ & $14 s$ & 0.45 & 0.42 & 0.39 & 0.39 & 0.39 & 0.40 & 3.82 & 3.90 & 4.17 & 4.11 & 4.12 & 4.12 \\
\hline $5 / 20 / 94$ & $14 t$ & 0.40 & 0.33 & 0.33 & 0.29 & 0.28 & 0.26 & 3.14 & 3.22 & 3.49 & 3.64 & 3.85 & 3.97 \\
\hline $5 / 23 / 94$ & $14 u$ & 0.03 & 0.01 & 0.01 & 0.00 & 0.00 & 0.00 & 2.52 & 4.11 & 5.33 & 6.13 & 6.88 & 7.49 \\
\hline $5 / 23 / 94$ & $14 v$ & 0.40 & 0.43 & 0.40 & 0.39 & 0.35 & 0.34 & 5.23 & 5.23 & 5.55 & 5.41 & 5.52 & 5.62 \\
\hline $5 / 23 / 94$ & $14 w$ & 0.32 & 0.31 & 0.33 & 0.32 & 0.33 & 0.33 & 5.63 & 5.21 & 4.80 & 4.70 & 4.41 & 4.03 \\
\hline $5 / 23 / 94$ & $14 x$ & 0.32 & 0.27 & 0.19 & 0.16 & 0.12 & 0.10 & 3.28 & 3.79 & 4.48 & 5.08 & 5.94 & 6.83 \\
\hline $5 / 23 / 94$ & $14 y$ & 0.27 & $\overline{0.14}$ & 0.07 & 0.05 & 0.04 & 0.03 & 2.82 & 3.95 & 5.47 & 6.18 & 6.63 & 7.37 \\
\hline $5 / 23 / 94$ & 142 & 0.03 & 0.01 & 0.01 & 0.01 & 0.00 & 0.00 & 3.40 & 5.15 & 6.32 & 7.11 & 7.61 & 8.23 \\
\hline $5 / 27 / 94$ & $15 a$ & 0.40 & 0.37 & 0.33 & 0.30 & 0.26 & 0.22 & 5.77 & 6.15 & 6.86 & 7.36 & 7.98 & 8.58 \\
\hline $5 / 27 / 94$ & $15 b$ & 0.37 & 0.28 & 0.22 & 0.17 & 0.13 & 0.11 & 4.52 & 5.19 & 6.33 & 7.36 & 8.26 & 9.00 \\
\hline $5 / 30 / 94$ & $15 c$ & 0.25 & 0.17 & 0.10 & 0.06 & 0.05 & 0.03 & 3.79 & 5.19 & 6.55 & 7.49 & 8.28 & 8.67 \\
\hline $5 / 30 / 94$ & $15 d$ & 0.12 & 0.06 & 0.04 & 0.02 & 0.01 & 0.01 & 3.90 & 5.73 & 6.93 & 7.38 & 8.46 & 8.84 \\
\hline $5 / 30 / 94$ & $15 \mathrm{e}$ & 0.01 & 0.01 & 0.00 & 0.00 & 0.00 & 0.00 & 4.11 & 5.68 & 8.07 & 8.61 & 11.26 & 11.25 \\
\hline $5 / 30 / 94$ & $15 f$ & 0.36 & 0.28 & 0.24 & 0.20 & 0.15 & 0.14 & 5.90 & 6.61 & 7.74 & 8.49 & 9.33 & 10.00 \\
\hline $5 / 30 / 94$ & $15 \mathrm{~g}$ & 0.35 & 0.24 & 0.18 & 0.13 & 0.09 & 0.08 & 4.73 & 5.85 & 7.39 & 8.46 & 9.27 & 10.21 \\
\hline $5 / 30 / 94$ & $15 \mathrm{~h}$ & 0.25 & 0.14 & 0.09 & 0.06 & 0.04 & 0.03 & 4.44 & 6.03 & 7.58 & 8.41 & 9.31 & 9.54 \\
\hline $5 / 30 / 94$ & $15 i$ & 0.11 & 0.05 & 0.03 & 0.02 & 0.01 & 0.01 & 4.35 & $\overline{6.10}$ & 7.14 & 8.35 & 8.94 & 9.46 \\
\hline $8 / 16 / 94$ & $17 d$ & 0.00 & 0.00 & 0.00 & 0.00 & 0.00 & 0.00 & & & & & & \\
\hline $8 / 16 / 94$ & $17 \mathrm{e}$ & 0.00 & 0.00 & 0.00 & 0.00 & 0.00 & 0.00 & & & & & & \\
\hline
\end{tabular}




\begin{tabular}{|c|c|c|c|c|c|c|c|c|c|c|c|c|c|}
\hline DATE & EXP & AVF2 & AVF3 & AVF4 & AVF5 & AVF6 & AVF7 & $\mathrm{S} 2 \mathrm{~V}$ & S3V & S4V & S5V & S6V & S7V \\
\hline $8 / 17 / 94$ & $17 \mathrm{f}$ & 0.00 & 0.00 & 0.00 & 0.00 & 0.00 & 0.00 & & & & & & \\
\hline $8 / 17 / 94$ & $17 g$ & 0.00 & 0.00 & 0.00 & 0.00 & 0.00 & 0.00 & & & & & & \\
\hline $8 / 18 / 94$ & $17 \mathrm{~h}$ & 0.02 & 0.01 & 0.01 & 0.00 & 0.00 & 0.00 & 4.27 & 4.96 & 6.77 & 7.83 & 8.13 & 8.07 \\
\hline $8 / 18 / 94$ & $17 j$ & 0.00 & 0.00 & 0.00 & 0.00 & 0.00 & 0.00 & & & & & & \\
\hline $8 / 18 / 94$ & $17 k$ & 0.00 & 0.00 & 0.00 & 0.00 & 0.00 & 0.00 & & & & & & \\
\hline $9 / 8 / 94$ & 171 & 0.00 & 0.00 & 0.00 & 0.00 & 0.00 & 0.00 & & & & & & \\
\hline $9 / 9 / 94$ & $17 \mathrm{~m}$ & 0.41 & 0.40 & 0.38 & 0.41 & 0.39 & 0.40 & 4.23 & 4.14 & 4.36 & 4.21 & 4.25 & 4.22 \\
\hline $9 / 12 / 94$ & $17 n$ & 0.00 & 0.00 & 0.00 & 0.00 & 0.00 & 0.00 & 0.00 & 0.00 & 0.00 & 0.00 & 0.00 & 0.00 \\
\hline $9 / 12 / 94$ & 170 & 0.00 & 0.00 & 0.00 & 0.00 & 0.00 & 0.00 & 0.00 & 0.00 & 0.00 & 0.00 & 0.00 & 0.00 \\
\hline $9 / 12 / 94$ & $17 p$ & 0.37 & 0.39 & 0.38 & 0.39 & 0.00 & 0.39 & 4.46 & 4.40 & 4.52 & 4.54 & 0.00 & 4.35 \\
\hline $9 / 12 / 94$ & $17 q$ & 0.40 & 0.40 & 0.38 & 0.39 & 0.38 & 0.40 & 4.34 & 4.33 & 4.49 & 4.40 & 4.38 & 4.27 \\
\hline $9 / 13 / 94$ & $17 r$ & 0.38 & 0.38 & 0.38 & 0.37 & 0.39 & 0.38 & 4.41 & 4.34 & 4.49 & 4.47 & 4.27 & 4.35 \\
\hline $9 / 13 / 94$ & $17 \mathrm{~s}$ & 0.38 & 0.38 & 0.37 & 0.37 & 0.37 & 0.38 & 4.40 & 4.25 & 4.46 & 4.39 & 4.35 & 4.35 \\
\hline $9 / 13 / 94$ & $17 t$ & 0.38 & 0.37 & 0.37 & 0.39 & 0.37 & 0.38 & 4.24 & 4.20 & 4.24 & 4.20 & 4.23 & 4.14 \\
\hline $9 / 14 / 94$ & $17 u$ & 0.36 & 0.38 & 0.37 & 0.36 & 0.37 & 0.37 & 4.61 & 4.45 & 4.57 & 4.60 & 4.52 & 4.54 \\
\hline $9 / 14 / 94$ & $17 v$ & 0.38 & 0.38 & 0.37 & 0.36 & 0.00 & 0.38 & 4.84 & 4.81 & 4.88 & 4.87 & 0.00 & 4.80 \\
\hline $9 / 14 / 94$ & $17 w$ & 0.36 & 0.35 & 0.36 & 0.36 & 0.35 & 0.36 & 4.53 & 4.50 & 4.58 & 4.57 & 4.64 & 4.47 \\
\hline $9 / 14 / 94$ & $17 x$ & 0.32 & 0.32 & 0.30 & $\overline{0.31}$ & 0.32 & 0.32 & 5.09 & 5.02 & 5.31 & 5.15 & 5.16 & 5.12 \\
\hline $9 / 14 / 94$ & $17 y$ & 0.28 & 0.28 & 0.27 & 0.27 & 0.26 & 0.28 & 4.92 & 5.03 & 5.14 & 5.15 & 5.24 & $\overline{5.12}$ \\
\hline $9 / 14 / 94$ & 172 & 0.31 & 0.32 & 0.31 & 0.32 & 0.31 & 0.32 & 4.83 & 4.80 & 4.94 & 4.91 & 4.94 & 4.78 \\
\hline $9 / 14 / 94$ & $18 a$ & 0.30 & 0.29 & 0.29 & 0.29 & 0.29 & 0.29 & 4.82 & 4.90 & 4.98 & 5.00 & 5.07 & 4.96 \\
\hline $9 / 14 / 94$ & $18 b$ & 0.30 & 0.30 & 0.29 & 0.30 & 0.29 & 0.30 & 4.80 & 4.81 & 4.90 & 4.87 & 4.90 & 4.73 \\
\hline $9 / 14 / 94$ & $18 c$ & 0.28 & 0.28 & 0.27 & 0.28 & 0.28 & 0.28 & 4.96 & 5.02 & 5.20 & 5.13 & 5.24 & 5.13 \\
\hline $9 / 14 / 94$ & $18 d$ & 0.27 & 0.26 & 0.27 & 0.27 & 0.29 & 0.29 & 10.73 & 10.73 & 10.58 & 10.60 & 10.11 & 9.91 \\
\hline $9 / 25 / 94$ & & 0.00 & 0.00 & 0.00 & 0.00 & 0.00 & 0.00 & & & & & & \\
\hline $10 / 6 / 94$ & $18 \mathrm{k}$ & 0.00 & 0.00 & 0.00 & 0.00 & 0.00 & 0.00 & & & & & & \\
\hline $10 / 6 / 94$ & 181 & 0.00 & 0.00 & 0.00 & 0.00 & 0.00 & 0.00 & & & & & & \\
\hline $10 / 7 / 94$ & $18 \mathrm{~m}$ & 0.00 & 0.00 & 0.00 & 0.00 & 0.00 & 0.00 & & & & & & \\
\hline $10 / 7 / 94$ & $18 n$ & 0.00 & 0.00 & 0.00 & 0.00 & 0.00 & 0.00 & & & & & & \\
\hline $10 / 10 / 94$ & 180 & 0.00 & 0.00 & 0.00 & 0.00 & 0.00 & 0.00 & & & & & & \\
\hline $10 / 10 / 94$ & $18 p$ & 0.00 & 0.00 & 0.00 & 0.00 & 0.00 & 0.00 & & & & & & \\
\hline $10 / 10 / 94$ & & 0.53 & 0.51 & 0.51 & 0.51 & 0.46 & 0.50 & 5.41 & 5.83 & 6.19 & 6.13 & 6.69 & 6.28 \\
\hline $10 / 10 / 94$ & & 0.57 & 0.54 & 0.53 & 0.53 & 0.52 & 0.49 & 0.35 & 0.39 & 0.43 & 0.45 & 0.50 & 0.63 \\
\hline $10 / 11 / 94$ & & 0.57 & 0.53 & 0.52 & 0.53 & 0.52 & 0.50 & 2.43 & 2.33 & 2.37 & 2.24 & 2.23 & 2.39 \\
\hline $10 / 14 / 94$ & $18 q$ & 0.00 & 0.00 & 0.00 & 0.00 & 0.00 & 0.00 & & & & & & \\
\hline $10 / 25 / 94$ & $18 \mathrm{~s}$ & 0.00 & 0.00 & 0.00 & 0.00 & 0.00 & 0.00 & & & & & & \\
\hline $10 / 26 / 94$ & $18 t$ & 0.00 & 0.00 & 0.00 & 0.00 & 0.00 & 0.00 & & & & & & \\
\hline
\end{tabular}


Data Summary

\begin{tabular}{|c|c|c|c|c|c|c|c|c|c|}
\hline DATE & EXP & S1MH & S2MH & S3MH & S4MH & S5MH & S6MH & S7MH & MDOT \\
\hline $1 / 19 / 93$ & $0 \mathrm{Ob}$ & 0.00 & 1.88 & 1.33 & 1.16 & 0.99 & 0.78 & 0.71 & 9.54 \\
\hline $1 / 19 / 93$ & $00 \mathrm{c}$ & 0.00 & 2.75 & 2.19 & 2.14 & 1.49 & 1.24 & 1.12 & 13.77 \\
\hline $1 / 19 / 93$ & $00 \mathrm{~d}$ & 0.00 & 4.48 & 3.60 & 3.16 & 2.75 & 2.17 & 2.05 & 17.65 \\
\hline $1 / 19 / 93$ & $00 \mathrm{e}$ & 0.00 & 4.61 & 4.13 & 3.69 & 3.35 & 2.84 & 2.77 & 20.20 \\
\hline $1 / 19 / 93$ & $00 f$ & 0.00 & 0.00 & 0.00 & 0.00 & 0.00 & 0.00 & 0.00 & 0.00 \\
\hline $1 / 21 / 93$ & $\overline{00 g}$ & 0.00 & 0.52 & 0.41 & 0.34 & 0.31 & 0.25 & 0.25 & 3.65 \\
\hline $1 / 21 / 93$ & OOh & .00 & 0.49 & 0.32 & 0.29 & 0.24 & 0.13 & 0.28 & 3.20 \\
\hline $1 / 25 / 93$ & $\overline{0 i}$ & 0.00 & 4.61 & 4.13 & 3.69 & 3.35 & 2.84 & 2.77 & 50.49 \\
\hline $1 / 27 / 93$ & $00 j$ & 0.00 & 3.95 & 3.20 & 2.87 & 2.47 & 2.16 & 1.91 & 25.50 \\
\hline $1 / 29 / 93$ & $00 \mathrm{~K}$ & 0.00 & 2.75 & 2.19 & 2.14 & 1.45 & 1.24 & 1.12 & 17.85 \\
\hline $1 / 31 / 93$ & 001 & 0.00 & 0.52 & 0.38 & 0.32 & 0.30 & 0.25 & 0.25 & 3.47 \\
\hline $1 / 31 / 93$ & $00 \mathrm{~m}$ & 0.00 & 1.64 & 1.23 & 1.04 & 0.90 & 0.77 & 0.70 & 10.71 \\
\hline $2 / 10 / 93$ & $00 n$ & 0.00 & 0.55 & 0.46 & 0.39 & 0.36 & 0.29 & 0.29 & 3.31 \\
\hline $2 / 10 / 93$ & 10 & 0.00 & 0.55 & 0.46 & 0.39 & 0.35 & 0.29 & 0.28 & 4.42 \\
\hline $1 / 93$ & & & 2.23 & 1.74 & .40 & 1.21 & 1.08 & 0.86 & 10.17 \\
\hline $2 / 11 / 93$ & $00 q$ & 0.00 & 2.20 & 1.75 & 1.40 & 1.27 & 1.02 & 0.89 & 10.20 \\
\hline 193 & $\overrightarrow{0 r}$ & 0.00 & 4.14 & 3.78 & 3.13 & 2.80 & 2.29 & 2.02 & 17.50 \\
\hline 193 & $00 \mathrm{~s}$ & 0.00 & 5.34 & 4.94 & 4.23 & 3.87 & 3.20 & 2.90 & 26.15 \\
\hline & & .00 & 5.25 & 5.31 & 4.94 & 4.79 & 4.31 & 4.05 & 45.08 \\
\hline$\sqrt{93}$ & $\overline{\mathrm{Ou}}$ & 0.00 & 5.25 & 5.31 & 4.94 & 4.79 & 4.31 & 4.05 & 45.08 \\
\hline $3 / 93$ & $00 \mathrm{~V}$ & 0.00 & 7.69 & 7.08 & 6.82 & 6.82 & 6.93 & 6.46 & 43.25 \\
\hline $2 / 24 / 93$ & $00 w$ & 0.00 & 1.83 & 1.80 & 1.78 & 1.92 & 1.88 & 1.71 & 1.35 \\
\hline $2 / 24 / 93$ & $00 x$ & 0.00 & 3.06 & 2.87 & 2.75 & 2.76 & 2.65 & 2.47 & 5.61 \\
\hline $2 / 24 / 93$ & $0 y$ & & 4.20 & 4.03 & 4.03 & 3.94 & 3.83 & 3.58 & 11.19 \\
\hline $4 / 93$ & & & 5.17 & 98 & 4.90 & 4.88 & 4.75 & 4.41 & 18.43 \\
\hline$\sqrt{93}$ & $01 a$ & 0.00 & 0.58 & 0.40 & 0.33 & 0.31 & 0.29 & 0.26 & 1.71 \\
\hline & $01 b$ & 0.00 & 2.42 & 1.70 & 1.32 & 1.12 & 1.01 & 0.86 & .39 \\
\hline & $01 c$ & 0.00 & 3.84 & 3.32 & 2.82 & 2.32 & 1.98 & 1.61 & 4.14 \\
\hline$\sqrt{93}$ & 01d & 0.00 & 4.95 & 4.48 & 4.03 & 3.69 & 3.26 & 2.75 & 19.18 \\
\hline 193 & $01 \mathrm{e}$ & 0.00 & 7.78 & 7.43 & 6.95 & 6.71 & 6.56 & 5.93 & 45.01 \\
\hline $2 / 93$ & $01 \mathrm{~g}$ & 0.00 & 2.69 & 2.17 & 1.87 & 1.65 & 1.50 & 1.32 & 11.15 \\
\hline$/ 193$ & $01 \mathrm{~h}$ & 0.00 & 4.30 & 3.92 & 3.56 & 3.26 & 2.92 & 2.49 & 21.22 \\
\hline 193 & $01 f$ & 0.00 & 0.72 & 0.65 & 0.58 & 0.56 & 0.54 & 0.46 & 3.26 \\
\hline t/93 & $01 k$ & & 5.29 & 4.90 & 5.13 & 5.48 & 5.45 & 4.84 & 21.49 \\
\hline $9 / 93$ & & & 3.31 & 3.17 & 3.52 & 3.11 & 2.77 & 3.38 & 6.43 \\
\hline & & & 4.08 & 4.39 & 4.28 & 3.90 & 4.30 & 4.02 & 10.40 \\
\hline 193 & & & 48 & 7.26 & 6.77 & 7.06 & 6.66 & 7.15 & 43.65 \\
\hline & & & 34 & & 68 & .67 & 0.68 & 0.56 & 2.89 \\
\hline & $n$ & & 3.51 & 80 & 3.49 & 3.41 & 4.38 & 3.23 & 8.26 \\
\hline & 10 & & 5.53 & 5.46 & 4.60 & 4.88 & 4.75 & 5.37 & 21.49 \\
\hline & & & 0.43 & 0.32 & 0.29 & 0.25 & 0.24 & 0.22 & 1.41 \\
\hline & $1 q$ & & 1.91 & 1.20 & 0.98 & 0.90 & 0.79 & 0.73 & 5.85 \\
\hline $3 / 15 / 93$ & $01 r$ & & 3.41 & 2.71 & 2.12 & 1.76 & 1.52 & 1.30 & 12.38 \\
\hline $3 / 15 / 93$ & $01 \mathrm{~s}$ & & 4.95 & 4.07 & 3.46 & 3.02 & 2.60 & 2.09 & 18.77 \\
\hline $3 / 15 / 93$ & $01 t$ & & 6.72 & 6.14 & 5.74 & 5.59 & 5.17 & 4.75 & 37.47 \\
\hline $3 / 15 / 93$ & $01 \mathrm{u}$ & & 0.75 & 0.66 & 0.58 & 0.55 & 0.52 & 0.43 & 3.94 \\
\hline $3 / 15 / 93$ & & & 2.59 & 2.00 & 1.60 & 1.41 & 1.25 & 1.12 & 11.70 \\
\hline $3 / 15 / 93$ & & & 3.48 & 2.98 & 2.53 & 2.25 & 1.98 & 1.88 & 18.22 \\
\hline & & & 5.97 & 5.32 & 5.15 & 5.06 & 5.19 & 4.37 & 3.74 \\
\hline $3 / 18 / 93$ & $01 y$ & 0.00 & 0.00 & 0.00 & 0.00 & 0.00 & 0.00 & 0.00 & 0.00 \\
\hline
\end{tabular}


Data Summary

\begin{tabular}{|c|c|c|c|c|c|c|c|c|c|}
\hline DATE & EXP & S1MH & S2MH & S3MH & S4MH & S5MH & S6MH & S7MH & MDOT \\
\hline $3 / 18 / 93$ & $01 z$ & 0.00 & 8.39 & 6.61 & 6.25 & 6.11 & 6.03 & 5.17 & 10.47 \\
\hline $3 / 18 / 93$ & $02 a$ & 0.00 & 5.49 & 5.60 & 5.95 & 5.55 & 5.07 & 4.02 & 3.47 \\
\hline $3 / 18 / 93$ & $02 b$ & 0.00 & 6.50 & 5.80 & 5.63 & 5.78 & 5.93 & 5.29 & 7.00 \\
\hline $3 / 18 / 93$ & $02 c$ & 0.00 & 11.39 & 8.88 & 8.36 & 7.80 & 7.84 & 7.40 & 23.05 \\
\hline $3 / 18 / 93$ & $02 d$ & 0.00 & 8.80 & 7.63 & 6.91 & 6.89 & 6.82 & 5.96 & 14.82 \\
\hline $3 / 19 / 93$ & $02 e$ & 0.00 & 10.91 & 8.65 & 8.05 & 7.77 & 7.97 & 7.08 & 11.22 \\
\hline $3 / 22 / 93$ & $02 f$ & 0.00 & 0.68 & 0.56 & 0.48 & 0.45 & 0.44 & 0.41 & 4.08 \\
\hline $3 / 22 / 93$ & $02 g$ & 0.00 & 1.81 & 1.36 & 1.16 & 1.05 & 0.97 & 0.86 & 12.58 \\
\hline $3 / 22 / 93$ & $02 \mathrm{~h}$ & 0.00 & 2.97 & 2.37 & 2.01 & 1.80 & 1.68 & 1.50 & 24.14 \\
\hline $3 / 22 / 93$ & $02 i$ & 0.00 & 0.37 & 0.29 & 0.25 & 0.25 & 0.25 & 0.22 & 1.95 \\
\hline $3 / 22 / 93$ & $09 z$ & 0.00 & 0.00 & 0.00 & 0.00 & 0.00 & 0.00 & 0.00 & 0.00 \\
\hline $3 / 23 / 93$ & $02 j$ & 0.00 & 1.06 & 0.83 & 0.69 & 0.65 & 0.61 & 0.56 & 6.53 \\
\hline $3 / 23 / 93$ & $02 k$ & 0.00 & 2.01 & 1.54 & 1.32 & 1.18 & 1.12 & 0.99 & 13.19 \\
\hline $3 / 23 / 93$ & 021 & 0.00 & 3.19 & 2.46 & 2.00 & 1.77 & 1.66 & 1.50 & 21.56 \\
\hline $3 / 23 / 93$ & $02 m$ & 0.00 & 4.24 & 3.45 & 2.94 & 2.69 & 2.47 & 2.20 & 34.17 \\
\hline $3 / 24 / 93$ & $02 n$ & 0.00 & 0.60 & 0.49 & 0.42 & 0.40 & 0.39 & 0.34 & 3.26 \\
\hline $3 / 24 / 93$ & 020 & 0.00 & 1.50 & 1.19 & 1.05 & 0.97 & 0.91 & 0.81 & 9.32 \\
\hline $3 / 24 / 93$ & $02 p$ & 0.00 & 3.22 & 2.56 & 2.17 & 1.99 & 1.85 & 1.58 & 22.58 \\
\hline $3 / 24 / 93$ & $02 q$ & 0.00 & 0.42 & 0.32 & 0.27 & 0.26 & 0.25 & 0.22 & 1.86 \\
\hline $3 / 24 / 93$ & $02 r$ & 0.00 & 1.30 & 0.93 & 0.80 & 0.72 & 0.69 & 0.66 & 6.94 \\
\hline $3 / 24 / 93$ & $02 \mathrm{~s}$ & 0.00 & 2.37 & 1.75 & 1.48 & 1.34 & 1.24 & 1.09 & 12.92 \\
\hline $3 / 24 / 93$ & $02 t$ & 0.00 & 3.65 & 2.75 & 2.28 & 1.99 & 1.85 & 1.58 & 20.20 \\
\hline $3 / 24 / 93$ & $02 u$ & 0.00 & 5.12 & 4.27 & 3.64 & 3.30 & 3.03 & 2.57 & 36.99 \\
\hline $3 / 24 / 93$ & $02 v$ & 0.00 & 0.41 & 0.31 & 0.27 & 0.25 & 0.24 & 0.21 & 1.78 \\
\hline $3 / 24 / 93$ & $02 w$ & 0.00 & 1.21 & 0.92 & 0.80 & 0.72 & 0.68 & 0.61 & 7.68 \\
\hline $3 / 25 / 93$ & $02 x$ & 0.00 & 2.29 & 1.70 & 1.46 & 1.30 & 1.22 & 1.02 & 13.33 \\
\hline $3 / 25 / 93$ & $02 y$ & 0.00 & 3.55 & 2.63 & 2.17 & 1.86 & 1.73 & 1.52 & 21.22 \\
\hline $3 / 25 / 93$ & $02 z$ & 0.00 & 4.84 & 3.97 & 3.40 & 3.06 & 2.78 & 2.37 & 37.26 \\
\hline $3 / 25 / 93$ & $\overline{03 a}$ & 0.00 & 0.62 & 0.47 & 0.42 & 0.39 & 0.37 & 0.36 & 2.92 \\
\hline $3 / 25 / 93$ & $03 \mathrm{~b}$ & 0.00 & 1.40 & 1.15 & 1.03 & 0.91 & 0.89 & 0.73 & 9.45 \\
\hline $3 / 25 / 93$ & $03 c$ & 0.00 & 3.33 & 2.59 & 2.21 & 2.00 & 1.88 & 1.65 & 25.02 \\
\hline $3 / 25 / 93$ & $03 d$ & 0.00 & 0.35 & 0.25 & 0.20 & 0.19 & 0.17 & 0.16 & 1.46 \\
\hline $3 / 25 / 93$ & $03 e$ & 0.00 & 1.38 & 0.92 & 0.78 & 0.71 & 0.66 & 0.58 & 6.37 \\
\hline $3 / 25 / 93$ & $03 \mathrm{f}$ & 0.00 & 2.67 & 1.88 & 1.51 & 1.32 & 1.22 & 1.05 & 12.44 \\
\hline $3 / 25 / 93$ & $03 \mathrm{~g}$ & 0.00 & 3.99 & 3.08 & 2.44 & 2.10 & 1.86 & 1.59 & 19.69 \\
\hline $3 / 25 / 93$ & $03 \mathrm{~h}$ & 0.00 & 5.62 & 4.88 & 4.25 & 3.81 & 3.44 & 2.92 & 37.06 \\
\hline $3 / 26 / 93$ & $03 i$ & 0.00 & 0.58 & 0.47 & 0.42 & 0.40 & 0.39 & 0.35 & 3.18 \\
\hline $3 / 26 / 93$ & 03j & 0.00 & 1.47 & 1.20 & 1.03 & 0.98 & 0.92 & 0.85 & 9.01 \\
\hline $3 / 26 / 93$ & $03 k$ & 0.00 & 3.36 & 2.70 & 2.29 & 2.06 & 1.90 & 1.69 & 21.42 \\
\hline $3 / 26 / 93$ & 031 & 0.00 & 0.42 & 0.30 & 0.25 & 0.23 & 0.21 & 0.19 & 1.29 \\
\hline $3 / 26 / 93$ & $03 \mathrm{~m}$ & 0.00 & 2.10 & 1.33 & 1.00 & 0.85 & 0.79 & 0.69 & 5.64 \\
\hline $3 / 26 / 93$ & $03 n$ & 0.00 & 3.52 & 2.97 & 2.40 & 1.97 & 1.63 & 1.38 & 11.22 \\
\hline $3 / 26 / 93$ & 030 & 0.00 & 4.51 & 4.10 & 3.75 & 3.52 & 3.10 & 2.56 & 17.31 \\
\hline $3 / 26 / 93$ & $03 p$ & 0.00 & 6.75 & 6.43 & 6.11 & 6.00 & 5.67 & 5.22 & 37.51 \\
\hline $3 / 26 / 93$ & $03 q$ & 0.00 & 0.55 & 0.47 & 0.41 & 0.40 & 0.37 & 0.34 & 2.69 \\
\hline $3 / 26 / 93$ & $03 r$ & 0.00 & 1.69 & 1.34 & 1.12 & 1.05 & 0.97 & 0.86 & 8.09 \\
\hline $3 / 26 / 93$ & $03 \mathrm{~s}$ & 0.00 & 3.85 & 3.52 & 3.13 & 2.86 & 2.70 & 2.12 & 18.97 \\
\hline $3 / 29 / 93$ & $03 t$ & 0.00 & 0.72 & 0.62 & 0.54 & 0.52 & 0.49 & 0.43 & 2.55 \\
\hline $3 / 29 / 93$ & $03 u$ & 0.00 & 3.69 & 3.62 & 3.73 & 4.50 & 3.01 & 3.64 & 6.66 \\
\hline $3 / 29 / 93$ & $03 v$ & 0.00 & 4.63 & 4.53 & 4.83 & 4.15 & 4.50 & 5.19 & 16.23 \\
\hline
\end{tabular}


Data Summary

\begin{tabular}{|c|c|c|c|c|c|c|c|c|c|}
\hline DATE & EXP & S1MH & $\mathrm{S} 2 \mathrm{MH}$ & S3MH & S4MH & S5MH & S6MH & S7MH & MDOT \\
\hline $3 / 29 / 93$ & $03 w$ & 0.00 & 0.00 & 0.00 & 0.00 & 0.00 & 0.00 & 0.00 & 0.00 \\
\hline $3 / 29 / 93$ & $03 x$ & 0.00 & 3.04 & 3.15 & 2.98 & 3.83 & 2.93 & 2.48 & 4.54 \\
\hline $3 / 29 / 93$ & O3y & 0.00 & 4.24 & 3.57 & 3.49 & $\overline{4.41}$ & 4.18 & 3.16 & 8.84 \\
\hline $3 / 29 / 93$ & $03 z$ & 0.00 & 5.37 & 4.33 & 4.53 & 4.47 & 3.98 & 4.28 & 14.76 \\
\hline $3 / 29 / 93$ & $04 a$ & 0.00 & 8.15 & 6.90 & 6.09 & 6.46 & 6.75 & 5.79 & 35.60 \\
\hline $3 / 30 / 93$ & $04 b$ & 0.00 & 3.95 & 4.12 & 4.29 & 4.38 & 3.99 & 3.34 & 1.80 \\
\hline $3 / 30 / 93$ & $04 c$ & 0.00 & 4.84 & 4.85 & 4.80 & 4.96 & 4.38 & 4.27 & 6.19 \\
\hline $3 / 30 / 93$ & 04d & 0.00 & 6.09 & 5.36 & 5.40 & 5.38 & 5.56 & 5.04 & 10.27 \\
\hline $3 / 30 / 93$ & $04 \mathrm{e}$ & 0.00 & 10.08 & 8.47 & 7.81 & 7.48 & 7.51 & 6.62 & 30.74 \\
\hline $3 / 30 / 93$ & $04 f$ & 0.00 & 4.31 & 4.26 & 4.29 & 4.40 & 4.46 & 3.94 & 2.55 \\
\hline $3 / 30 / 93$ & $04 \mathrm{~g}$ & 0.00 & 5.20 & 5.08 & 4.97 & 5.18 & 5.36 & 4.61 & 8.50 \\
\hline $3 / 30 / 93$ & $04 \mathrm{~h}$ & 0.00 & 5.93 & 6.11 & 6.17 & 5.95 & 6.07 & 5.92 & 13.50 \\
\hline $3 / 30 / 93$ & $04 i$ & 0.00 & 11.89 & 9.19 & 8.05 & 7.87 & 8.10 & 7.70 & 10.13 \\
\hline $3 / 30 / 93$ & $04 j$ & 0.00 & 10.61 & 8.12 & 7.80 & 8.07 & 8.44 & 7.58 & 9.55 \\
\hline $4 / 1 / 93$ & $04 n$ & 0.00 & 5.61 & 4.98 & 4.64 & 5.31 & 5.51 & 4.73 & 4.21 \\
\hline $4 / 1 / 93$ & $04 k$ & 0.00 & 4.80 & 4.96 & 4.55 & 4.83 & 4.79 & 4.35 & 7.44 \\
\hline $4 / 1 / 93$ & 041 & 0.00 & 5.50 & 6.65 & 6.19 & 6.56 & 6.80 & 6.21 & 37.03 \\
\hline $4 / 1 / 93$ & $04 \mathrm{~m}$ & 0.00 & 6.19 & 4.55 & 4.43 & 4.59 & 4.56 & 4.26 & 17.77 \\
\hline $4 / 1 / 93$ & 040 & 0.00 & 9.41 & 7.84 & 6.74 & 6.69 & 6.27 & 6.22 & 33.25 \\
\hline $4 / 1 / 93$ & $04 p$ & 0.00 & 5.01 & 5.10 & 5.14 & 5.60 & 5.72 & 5.45 & 14.96 \\
\hline $4 / 1 / 93$ & $04 q$ & 0.00 & 8.37 & 7.34 & 7.47 & 7.41 & 7.06 & 6.86 & 3.49 \\
\hline $4 / 2 / 93$ & $04 r$ & 0.00 & 4.47 & 3.83 & 3.23 & 2.93 & 2.69 & 2.22 & 36.18 \\
\hline $4 / 5 / 93$ & $04 \mathrm{~s}$ & 0.00 & 2.25 & 1.53 & 1.28 & 0.00 & 0.00 & 0.97 & 13.80 \\
\hline $4 / 5 / 93$ & $04 t$ & 0.00 & 1.30 & 0.97 & 0.83 & 0.77 & 0.73 & 0.64 & .25 \\
\hline $4 / 5 / 93$ & $04 u$ & 0.00 & 3.20 & 2.59 & 0.00 & 1.91 & 0.00 & 1.49 & 25.30 \\
\hline $4 / 5 / 93$ & $04 \mathrm{v}$ & 0.00 & 0.59 & 0.00 & 0.00 & 0.00 & 0.00 & 0.24 & 2.38 \\
\hline $4 / 5 / 93$ & $04 w$ & 0.00 & 1.98 & 1.69 & 1.39 & 1.24 & 1.14 & 0.95 & 7.11 \\
\hline $4 / 5 / 93$ & $04 x$ & 0.00 & 0.00 & 0.00 & 0.00 & 0.00 & 0.00 & 0.00 & 0.00 \\
\hline $4 / 5 / 93$ & $04 y$ & 0.00 & 3.51 & 3.77 & 3.83 & 3.74 & 3.88 & 3.51 & 2.92 \\
\hline $4 / 5 / 93$ & $04 z$ & 0.00 & 4.46 & 4.42 & 4.27 & 4.17 & 4.26 & 3.87 & 7.99 \\
\hline $4 / 5 / 93$ & $05 a$ & & 3.97 & 4.06 & 4.07 & 4.24 & 4.33 & .78 & 9.08 \\
\hline $4 / 5 / 93$ & $05 b$ & 0.00 & 5.99 & 5.07 & 4.34 & 4.74 & 5.36 & 4.95 & 12.07 \\
\hline $4 / 7 / 93$ & $05 c$ & 0.00 & 6.23 & 6.25 & 6.12 & 5.93 & 5.50 & 4.92 & 36.99 \\
\hline $4 / 7 / 93$ & $05 d$ & 0.00 & 0.53 & 0.42 & 0.36 & 0.35 & 0.33 & 0.35 & 2.48 \\
\hline $4 / 7 / 93$ & $05 \mathrm{e}$ & 0.00 & 1.50 & 1.25 & 1.11 & 1.03 & 0.98 & 0.86 & 7.60 \\
\hline $4 / 7 / 93$ & $05 f$ & 0.00 & 3.91 & 3.57 & 3.15 & 2.85 & 2.58 & 2.16 & 19.62 \\
\hline $4 / 12 / 93$ & $05 \mathrm{~g}$ & 0.00 & 5.72 & 5.14 & 5.00 & 5.09 & 5.13 & 4.78 & 11.42 \\
\hline $4 / 12 / 93$ & $05 \mathrm{~h}$ & 0.00 & 0.00 & 0.00 & 0.00 & 0.00 & 0.00 & 0.00 & 2.44 \\
\hline $6 / 14 / 93$ & $05 n$ & 0.00 & 9.58 & 7.75 & 6.72 & 6.50 & 6.56 & 5.91 & 33.18 \\
\hline $6 / 14 / 93$ & 050 & & 5.98 & 4.96 & 4.65 & 5.18 & 5.46 & 4.98 & 0.78 \\
\hline $6 / 14 / 93$ & $05 p$ & 0.00 & 5.57 & 4.48 & 4.44 & 4.52 & 4.90 & 4.25 & 3.70 \\
\hline $6 / 14 / 93$ & $05 q$ & 0.00 & 5.70 & 4.53 & 4.31 & 4.58 & 4.47 & 4.16 & 1.80 \\
\hline $6 / 14 / 93$ & $05 r$ & 0.00 & 0.00 & 0.00 & 0.00 & 0.00 & 0.00 & 0.00 & 13.19 \\
\hline $6 / 14 / 93$ & $05 \mathrm{~s}$ & 0.00 & 0.00 & 0.00 & 0.00 & 0.00 & 0.00 & 0.00 & 7.50 \\
\hline $6 / 15 / 93$ & $05 t$ & 0.00 & 0.00 & 0.00 & 0.00 & 0.00 & 0.00 & 0.00 & 49.00 \\
\hline $6 / 15 / 93$ & $05 u$ & 0.00 & 0.00 & 0.00 & 0.00 & 0.00 & 0.00 & 0.00 & 12.34 \\
\hline $6 / 16 / 93$ & $05 \mathrm{v}$ & 0.00 & 0.00 & 0.00 & 0.00 & 0.00 & 0.00 & 0.00 & 0.00 \\
\hline $6 / 17 / 93$ & $05 w$ & 0.00 & 0.00 & 0.00 & 0.00 & 0.00 & 0.00 & 0.00 & 12.38 \\
\hline $6 / 17 / 93$ & $05 x$ & 0.00 & 0.00 & 0.00 & 0.00 & 0.00 & 0.00 & 0.00 & 7.20 \\
\hline $6 / 17 / 93$ & $05 y$ & 0.00 & 0.00 & 0.00 & 0.00 & 0.00 & 0.00 & 0.00 & 43.47 \\
\hline
\end{tabular}




\begin{tabular}{|c|c|c|c|c|c|c|c|c|c|}
\hline DATE & EXP & S1MH & S2MH & S3MH & S4MH & S5MH & S6MH & S7MH & MDOT \\
\hline $6 / 17 / 93$ & $05 z$ & 0.00 & 0.00 & 0.00 & 0.00 & 0.00 & 0.00 & 0.00 & 34.97 \\
\hline $6 / 28 / 93$ & $06 a$ & 0.00 & 0.00 & 0.00 & 0.00 & 0.00 & 0.00 & 0.00 & 0.00 \\
\hline $7 / 1 / 93$ & $06 b$ & 0.00 & 0.00 & 0.00 & 0.00 & 0.00 & 0.00 & 0.00 & 26.69 \\
\hline $7 / 1 / 93$ & $06 c$ & 0.00 & 0.00 & 0.00 & 0.00 & 0.00 & 0.00 & 0.00 & 32.16 \\
\hline $7 / 1 / 93$ & 06d & 0.00 & 0.00 & 0.00 & 0.00 & 0.00 & 0.00 & 0.00 & 25.47 \\
\hline $7 / 1 / 93$ & $06 \mathrm{e}$ & 0.00 & 0.00 & 0.00 & 0.00 & 0.00 & 0.00 & 0.00 & 30.74 \\
\hline $7 / 6 / 93$ & $06 f$ & 0.00 & 0.00 & 0.00 & 0.00 & 0.00 & 0.00 & 0.00 & 6.87 \\
\hline $7 / 6 / 93$ & $06 \mathrm{~h}$ & 0.00 & 0.00 & 0.00 & 0.00 & 0.00 & 0.00 & 0.00 & 16.80 \\
\hline $7 / 8 / 93$ & $06 \mathrm{~g}$ & 0.00 & 0.00 & 0.00 & 0.00 & 0.00 & 0.00 & 0.00 & 7.00 \\
\hline $7 / 8 / 93$ & $06 i$ & 0.00 & 0.00 & 0.00 & 0.00 & 0.00 & 0.00 & 0.00 & 12.24 \\
\hline $7 / 8 / 93$ & $06 j$ & 0.00 & 0.00 & 0.00 & 0.00 & 0.00 & 0.00 & 0.00 & 30.33 \\
\hline $7 / 8 / 93$ & $06 k$ & 0.00 & 0.00 & 0.00 & 0.00 & 0.00 & 0.00 & 0.00 & 21.56 \\
\hline $7 / 8 / 93$ & 061 & 0.00 & 0.00 & 0.00 & 0.00 & 0.00 & 0.00 & 0.00 & 31.94 \\
\hline $7 / 8 / 93$ & $06 \mathrm{~m}$ & 0.00 & 0.00 & 0.00 & 0.00 & 0.00 & 0.00 & 0.00 & 26.04 \\
\hline $7 / 8 / 93$ & $06 n$ & 0.00 & 0.00 & 0.00 & 0.00 & 0.00 & 0.00 & 0.00 & 28.81 \\
\hline $7 / 8 / 93$ & 060 & 0.00 & 0.00 & 0.00 & 0.00 & 0.00 & 0.00 & 0.00 & 19.11 \\
\hline $7 / 12 / 93$ & $06 p$ & 0.00 & 0.00 & 0.00 & 0.00 & 0.00 & 0.00 & 0.00 & 31.46 \\
\hline $7 / 12 / 93$ & $06 q$ & 0.00 & 0.00 & 0.00 & 0.00 & 0.00 & 0.00 & 0.00 & 12.75 \\
\hline $7 / 12 / 93$ & $06 r$ & 0.00 & 0.00 & 0.00 & 0.00 & 0.00 & 0.00 & 0.00 & 31.21 \\
\hline $7 / 12 / 93$ & $06 s$ & 0.00 & 0.00 & 0.00 & 0.00 & 0.00 & 0.00 & 0.00 & 11.53 \\
\hline $7 / 12 / 93$ & $06 t$ & 0.00 & 0.00 & 0.00 & 0.00 & 0.00 & 0.00 & 0.00 & 30.57 \\
\hline $7 / 12 / 93$ & $06 u$ & 0.00 & 0.00 & 0.00 & 0.00 & 0.00 & 0.00 & 0.00 & 11.25 \\
\hline $7 / 12 / 93$ & $06 v$ & 0.00 & 0.00 & 0.00 & 0.00 & 0.00 & 0.00 & 0.00 & 29.38 \\
\hline $7 / 13 / 93$ & $06 w$ & 0.00 & 0.00 & 0.00 & 0.00 & 0.00 & 0.00 & 0.00 & 14.76 \\
\hline $7 / 13 / 93$ & $06 x$ & 0.00 & 0.00 & 0.00 & 0.00 & 0.00 & 0.00 & 0.00 & 30.23 \\
\hline $7 / 13 / 93$ & $06 y$ & 0.00 & 0.00 & 0.00 & 0.00 & 0.00 & 0.00 & 0.00 & 13.69 \\
\hline $7 / 13 / 93$ & $06 z$ & 0.00 & 0.00 & 0.00 & 0.00 & 0.00 & 0.00 & 0.00 & 30.84 \\
\hline $7 / 17 / 93$ & $\overline{07 a}$ & 0.00 & 0.00 & 0.00 & 0.00 & 0.00 & 0.00 & 0.00 & 9.66 \\
\hline $7 / 17 / 93$ & 07b & 0.00 & 0.00 & 0.00 & 0.00 & 0.00 & 0.00 & 0.00 & 27.20 \\
\hline $7 / 17 / 93$ & $07 c$ & 0.00 & 0.00 & 0.00 & 0.00 & 0.00 & 0.00 & 0.00 & 9.13 \\
\hline $7 / 19 / 93$ & 07d & 0.00 & 0.00 & 0.00 & 0.00 & 0.00 & 0.00 & 0.00 & 28.08 \\
\hline $7 / 19 / 93$ & $\overline{07 e}$ & 0.00 & 0.00 & 0.00 & 0.00 & 0.00 & 0.00 & 0.00 & 0.00 \\
\hline $7 / 19 / 93$ & $07 f$ & 0.00 & 0.00 & 0.00 & 0.00 & 0.00 & 0.00 & 0.00 & 0.00 \\
\hline $7 / 19 / 93$ & $07 \mathrm{~g}$ & 0.00 & 0.00 & 0.00 & 0.00 & 0.00 & 0.00 & 0.00 & 10.10 \\
\hline $7 / 19 / 93$ & $07 \mathrm{~h}$ & 0.00 & 0.00 & 0.00 & 0.00 & 0.00 & 0.00 & 0.00 & 26.83 \\
\hline $7 / 20 / 93$ & $07 i$ & 0.00 & 0.00 & 0.00 & 0.00 & 0.00 & 0.00 & 0.00 & 15.52 \\
\hline $7 / 20 / 93$ & $07 j$ & 0.00 & 0.00 & 0.00 & 0.00 & 0.00 & 0.00 & 0.00 & 27.10 \\
\hline $7 / 20 / 93$ & $07 k$ & 0.00 & 0.00 & 0.00 & 0.00 & 0.00 & 0.00 & 0.00 & 7.00 \\
\hline $7 / 20 / 93$ & 071 & 0.00 & 0.00 & 0.00 & 0.00 & 0.00 & 0.00 & 0.00 & 25.78 \\
\hline $7 / 20 / 93$ & $07 \mathrm{~m}$ & 0.00 & 0.00 & 0.00 & 0.00 & 0.00 & 0.00 & 0.00 & 6.12 \\
\hline $7 / 20 / 93$ & $07 n$ & 0.00 & 0.00 & 0.00 & 0.00 & 0.00 & 0.00 & 0.00 & 22.73 \\
\hline $7 / 28 / 93$ & 070 & 0.00 & 0.00 & 0.00 & 0.00 & 0.00 & 0.00 & 0.00 & 31.56 \\
\hline $7 / 28 / 93$ & $07 p$ & 0.00 & 0.00 & 0.00 & 0.00 & 0.00 & 0.00 & 0.00 & 25.50 \\
\hline $7 / 28 / 93$ & $07 q$ & 0.00 & 0.00 & 0.00 & 0.00 & 0.00 & 0.00 & 0.00 & 29.95 \\
\hline $7 / 29 / 93$ & $07 r$ & 0.00 & 0.00 & 0.00 & 0.00 & 0.00 & 0.00 & 0.00 & 29.35 \\
\hline $7 / 29 / 93$ & $07 \mathrm{~s}$ & 0.00 & 0.00 & 0.00 & 0.00 & 0.00 & 0.00 & 0.00 & 29.31 \\
\hline $7 / 29 / 93$ & $07 t$ & 0.00 & 0.00 & 0.00 & 0.00 & 0.00 & 0.00 & 0.00 & 20.57 \\
\hline $8 / 4 / 93$ & $07 u$ & 0.00 & 0.00 & 0.00 & 0.00 & 0.00 & 0.00 & 0.00 & 6.13 \\
\hline $8 / 4 / 93$ & $07 v$ & 0.00 & 0.00 & 0.00 & 0.00 & 0.00 & 0.00 & 0.00 & 25.08 \\
\hline $8 / 5 / 93$ & $07 w$ & 0.00 & 0.00 & 0.00 & 0.00 & 0.00 & 0.00 & 0.00 & 24.15 \\
\hline
\end{tabular}


Data Summary

\begin{tabular}{|c|c|c|c|c|c|c|c|c|c|}
\hline DATE & EXP & S1MH & S2MH & S3MH & S4MH & S5MH & S6MH & S7MH & MDOT \\
\hline $8 / 5 / 93$ & $07 x$ & 0.00 & 0.00 & 0.00 & 0.00 & 0.00 & 0.00 & 0.00 & 29.44 \\
\hline $8 / 5 / 93$ & $07 y$ & 0.00 & 0.00 & 0.00 & 0.00 & 0.00 & 0.00 & 0.00 & 24.24 \\
\hline $8 / 12 / 93$ & $07 z$ & 0.00 & 0.00 & 0.00 & 0.00 & 0.00 & 0.00 & 0.00 & 29.39 \\
\hline $8 / 12 / 93$ & $08 a$ & 0.00 & 0.00 & 0.00 & 0.00 & 0.00 & 0.00 & 0.00 & 3.35 \\
\hline $8 / 12 / 93$ & $08 b$ & 0.00 & 0.00 & 0.00 & 0.00 & 0.00 & 0.00 & 0.00 & 13.44 \\
\hline $8 / 17 / 93$ & $08 \mathrm{c}$ & 0.00 & 6.20 & 5.39 & 5.20 & 5.74 & 5.63 & 4.85 & 12.53 \\
\hline $8 / 17 / 93$ & $08 \mathrm{~d}$ & 0.00 & 0.00 & 0.00 & 0.00 & 0.00 & 0.00 & 0.00 & 0.00 \\
\hline $8 / 17 / 93$ & $08 e$ & 0.00 & 0.00 & 0.00 & 0.00 & 0.00 & 0.00 & 0.00 & 0.00 \\
\hline $8 / 17 / 93$ & $08 f$ & 0.00 & 0.00 & 0.00 & 0.00 & 0.00 & 0.00 & 0.00 & 0.00 \\
\hline $8 / 17 / 93$ & $08 \mathrm{~g}$ & 0.00 & 0.00 & 0.00 & 0.00 & 0.00 & 0.00 & 0.00 & 0.00 \\
\hline $8 / 17 / 93$ & $08 \mathrm{~h}$ & 0.00 & 0.00 & 0.00 & 0.00 & 0.00 & 0.00 & 0.00 & 0.00 \\
\hline $8 / 17 / 93$ & $08 i$ & 0.00 & 0.00 & 0.00 & 0.00 & 0.00 & 0.00 & 0.00 & 0.00 \\
\hline $8 / 17 / 93$ & $08 j$ & 0.00 & 0.00 & 0.00 & 0.00 & 0.00 & 0.00 & 0.00 & 0.00 \\
\hline $8 / 31 / 93$ & $08 \mathrm{k}$ & 0.00 & 5.41 & 4.59 & 4.53 & 5.02 & 5.26 & 4.67 & 8.31 \\
\hline $9 / 1 / 93$ & 081 & 0.00 & 6.15 & 4.59 & 5.00 & 4.86 & 4.97 & 5.02 & 10.83 \\
\hline $9 / 1 / 93$ & $08 \mathrm{~m}$ & 0.00 & 5.23 & 4.49 & 4.56 & 4.99 & 5.18 & 4.48 & 9.67 \\
\hline $9 / 1 / 93$ & $08 n$ & 0.00 & 6.12 & 4.82 & 4.58 & 5.04 & 5.44 & 4.63 & 12.48 \\
\hline $9 / 2 / 93$ & 080 & 0.00 & 5.69 & 4.58 & 4.46 & 5.09 & 4.91 & 4.38 & 12.78 \\
\hline $9 / 2 / 93$ & $08 p$ & 0.00 & 5.09 & 4.48 & 4.32 & 4.67 & 4.56 & 4.05 & 10.28 \\
\hline $9 / 2 / 93$ & $08 q$ & 0.00 & 6.07 & 4.82 & 4.38 & 4.38 & 4.60 & 4.16 & 13.53 \\
\hline $9 / 2 / 93$ & $08 r$ & 0.00 & 5.30 & 4.35 & 4.29 & 4.66 & 4.81 & 4.33 & 10.81 \\
\hline $9 / 6 / 93$ & 085 & 0.00 & 5.61 & 4.71 & 4.23 & 4.64 & 4.91 & 3.81 & 14.04 \\
\hline $9 / 6 / 93$ & $08 t$ & 0.00 & 4.58 & 3.97 & 4.02 & 4.16 & 4.33 & 3.90 & 11.19 \\
\hline 9/6/93 & $08 u$ & 0.00 & 5.12 & 4.40 & 4.52 & 4.66 & 5.08 & 4.52 & 15.08 \\
\hline $9 / 6 / 93$ & $08 \mathrm{v}$ & 0.00 & 4.56 & 4.09 & 4.21 & 4.08 & 4.92 & 3.85 & 11.64 \\
\hline $9 / 6 / 93$ & $08 w$ & 0.00 & 5.20 & 4.49 & 4.37 & 5.06 & 4.28 & 4.10 & 14.18 \\
\hline $9 / 6 / 93$ & $08 x$ & 0.00 & 4.53 & 4.32 & 4.05 & 4.32 & 3.91 & 3.62 & 11.39 \\
\hline $9 / 7 / 93$ & $08 y$ & 0.00 & 5.33 & 4.41 & 4.24 & 4.64 & 4.89 & 4.42 & 15.37 \\
\hline $9 / 7 / 93$ & $08 z$ & 0.00 & 4.37 & 4.04 & 3.84 & 4.73 & 4.70 & 3.68 & 12.34 \\
\hline $9 / 7 / 93$ & $09 a$ & 0.00 & 4.97 & 4.30 & 4.47 & 4.44 & 4.75 & 4.35 & 15.71 \\
\hline $9 / 7 / 93$ & $09 b$ & 0.00 & 4.31 & 4.18 & 3.95 & 4.05 & 4.30 & 3.73 & 12.73 \\
\hline $9 / 7 / 93$ & $09 c$ & 0.00 & 6.04 & 5.04 & 4.74 & 5.20 & 5.44 & 4.80 & 11.19 \\
\hline $9 / 7 / 93$ & 09d & 0.00 & 5.07 & 4.63 & 4.30 & 4.92 & 5.09 & 4.12 & 9.88 \\
\hline $9 / 8 / 93$ & $\overline{09 e}$ & 0.00 & 5.76 & 4.48 & 4.82 & 5.27 & 5.27 & 4.42 & 10.86 \\
\hline 9/8/93 & $09 f$ & 0.00 & 5.13 & 5.01 & 5.16 & 4.82 & 5.11 & 4.44 & 8.72 \\
\hline $9 / 13 / 93$ & $09 g$ & 0.00 & 5.05 & 4.47 & 4.75 & 4.73 & 4.85 & 4.45 & 16.37 \\
\hline $9 / 13 / 93$ & $09 \mathrm{~g}$ & 0.00 & 5.05 & 4.47 & 4.75 & 4.73 & 4.85 & 4.45 & 15.32 \\
\hline $9 / 13 / 93$ & $09 \mathrm{~h}$ & 0.00 & 4.18 & 4.16 & 3.74 & 4.79 & 4.79 & 3.64 & 12.43 \\
\hline $9 / 14 / 93$ & $09 i$ & 0.00 & 4.63 & 4.82 & 4.63 & 4.47 & 4.50 & 4.26 & 16.34 \\
\hline $9 / 14 / 93$ & $09 i$ & 0.00 & 4.67 & 4.56 & 4.45 & 4.51 & 4.61 & 4.37 & 16.42 \\
\hline $9 / 14 / 93$ & $09 j$ & 0.00 & 4.02 & 4.09 & 4.24 & 4.19 & 4.22 & 3.94 & 13.43 \\
\hline $9 / 14 / 93$ & $09 k$ & 0.00 & 4.72 & 4.43 & 4.38 & 4.45 & 4.47 & 4.26 & 16.76 \\
\hline $9 / 14 / 93$ & 091 & 0.00 & 4.00 & 4.21 & 4.07 & 4.02 & 3.98 & 3.72 & 13.26 \\
\hline $9 / 14 / 93$ & $09 m$ & 0.00 & 4.47 & 4.40 & 4.44 & 4.37 & 4.16 & 3.82 & 17.03 \\
\hline $9 / 15 / 93$ & $09 n$ & 0.00 & 4.17 & 3.96 & 3.76 & 3.76 & 3.61 & 3.32 & 13.74 \\
\hline $9 / 17 / 93$ & 090 & 0.00 & 4.70 & 4.27 & 4.25 & $4 . \overline{38}$ & 4.25 & 3.87 & 16.74 \\
\hline $9 / 17 / 93$ & $09 p$ & 0.00 & 4.55 & 4.58 & 4.36 & 4.37 & 4.29 & 3.81 & 17.07 \\
\hline $9 / 17 / 93$ & $09 q$ & 0.00 & 4.49 & 4.41 & 4.40 & 4.35 & 4.35 & 3.91 & 17.12 \\
\hline $9 / 20 / 93$ & $09 r$ & 0.00 & 4.71 & 4.36 & 4.31 & 4.31 & 4.56 & 3.98 & 16.93 \\
\hline $9 / 20 / 93$ & $09 \mathrm{~s}$ & 0.00 & 4.51 & 4.52 & 4.33 & 4.35 & 4.63 & 3.99 & 16.91 \\
\hline
\end{tabular}


Data Summary

\begin{tabular}{|c|c|c|c|c|c|c|c|c|c|}
\hline DATE & EXP & S1MH & $\mathrm{S} 2 \mathrm{MH}$ & S3MH & S4MH & S5MH & S6MH & S7MH & MDOT \\
\hline $9 / 20 / 93$ & $09 t$ & 0.00 & 4.51 & 4.39 & 4.37 & 4.44 & 4.33 & 3.87 & 16.57 \\
\hline 9/20/93 & $09 u$ & 0.00 & 4.35 & 4.60 & 4.37 & 0.00 & 4.55 & 3.95 & 17.15 \\
\hline $9 / 21 / 93$ & $09 v$ & 0.00 & 4.72 & 4.52 & 4.15 & 4.14 & 4.33 & 3.76 & 16.86 \\
\hline $9 / 21 / 93$ & $09 w$ & 0.00 & 4.49 & 4.37 & 4.21 & 4.36 & 4.74 & 3.91 & 17.22 \\
\hline $9 / 21 / 93$ & $09 x$ & 0.00 & 4.57 & 4.62 & 4.12 & 4.22 & 4.87 & 3.86 & 17.07 \\
\hline $9 / 22 / 93$ & $09 y$ & 0.00 & 0.00 & 0.00 & 0.00 & 0.00 & 0.00 & 0.00 & 0.00 \\
\hline $9 / 22 / 93$ & $10 a$ & 0.00 & 0.00 & 0.00 & 0.00 & 0.00 & 0.00 & 0.00 & 0.00 \\
\hline $9 / 22 / 93$ & $10 \mathrm{~b}$ & 0.00 & 0.00 & 0.00 & 0.00 & 0.00 & 0.00 & 0.00 & 0.00 \\
\hline $9 / 22 / 93$ & $10 \mathrm{c}$ & 0.00 & 0.00 & 0.00 & 0.00 & 0.00 & 0.00 & 0.00 & 0.00 \\
\hline $9 / 23 / 93$ & $10 \mathrm{~d}$ & 0.00 & 0.00 & 0.00 & 0.00 & 0.00 & 0.00 & 0.00 & 0.00 \\
\hline $9 / 23 / 93$ & $10 \mathrm{e}$ & 0.00 & 0.00 & 0.00 & 0.00 & 0.00 & 0.00 & 0.00 & 0.00 \\
\hline $9 / 23 / 93$ & $10 f$ & 0.00 & 0.00 & 0.00 & 0.00 & 0.00 & 0.00 & 0.00 & 0.00 \\
\hline $9 / 23 / 93$ & $10 \mathrm{~g}$ & 0.00 & 0.00 & 0.00 & 0.00 & 0.00 & 0.00 & 0.00 & 0.00 \\
\hline $9 / 23 / 93$ & $10 \mathrm{~h}$ & 0.00 & 0.00 & 0.00 & 0.00 & 0.00 & 0.00 & 0.00 & 0.00 \\
\hline $9 / 23 / 93$ & $10 i$ & 0.00 & 0.00 & 0.00 & 0.00 & 0.00 & 0.00 & 0.00 & 0.00 \\
\hline $9 / 24 / 93$ & $10 j$ & 0.00 & 0.00 & 0.00 & 0.00 & 0.00 & 0.00 & 0.00 & 0.00 \\
\hline $9 / 24 / 93$ & $10 \mathrm{k}$ & 0.00 & 0.00 & 0.00 & 0.00 & 0.00 & 0.00 & 0.00 & 0.00 \\
\hline $9 / 24 / 93$ & 101 & 0.00 & 0.00 & 0.00 & 0.00 & 0.00 & 0.00 & 0.00 & 0.00 \\
\hline $9 / 24 / 93$ & $10 \mathrm{~m}$ & 0.00 & 0.00 & 0.00 & 0.00 & 0.00 & 0.00 & 0.00 & 0.00 \\
\hline $10 / 6 / 93$ & $10 n$ & 0.00 & 4.65 & 4.51 & 4.33 & 4.48 & 4.95 & 3.95 & 16.91 \\
\hline $10 / 6 / 93$ & 100 & 0.00 & 4.42 & 4.58 & 4.27 & 4.36 & 4.85 & 4.02 & 16.74 \\
\hline $10 / 7 / 93$ & $10 p$ & 0.00 & 4.72 & 4.65 & 4.35 & 4.32 & 4.57 & 4.13 & 17.00 \\
\hline $10 / 11 / 93$ & $10 q$ & 0.00 & 4.71 & 4.70 & 4.53 & 4.69 & 4.61 & 4.09 & 17.19 \\
\hline $10 / 11 / 93$ & $10 r$ & 0.00 & 4.55 & 4.53 & 4.47 & 4.68 & 4.86 & 4.23 & 16.47 \\
\hline $10 / 12 / 93$ & $10 \mathrm{~s}$ & 0.00 & 4.66 & 4.46 & 4.35 & 4.46 & 4.68 & 4.22 & 17.31 \\
\hline $10 / 12 / 93$ & $10 t$ & 0.00 & 4.62 & 4.35 & 4.46 & 4.66 & 5.01 & 4.15 & 16.64 \\
\hline $10 / 12 / 93$ & $10 u$ & 0.00 & 4.82 & 4.35 & 4.44 & 5.48 & 6.09 & 4.51 & 16.85 \\
\hline $10 / 13 / 93$ & $10 w$ & 0.00 & 4.58 & 4.61 & 4.35 & 4.59 & 5.10 & 4.33 & 16.81 \\
\hline $10 / 14 / 93$ & $10 y$ & 0.00 & 4.08 & 4.47 & 4.33 & 4.07 & 4.38 & 4.56 & 17.20 \\
\hline $10 / 18 / 93$ & $10 z$ & 0.00 & 5.33 & 4.42 & 4.21 & 4.84 & 4.72 & 4.42 & 13.79 \\
\hline $10 / 18 / 93$ & $11 \mathrm{a}$ & 0.00 & 5.64 & 4.84 & 4.52 & 4.96 & 4.24 & 4.20 & 14.18 \\
\hline $10 / 18 / 93$ & $11 b$ & 0.00 & 5.50 & 4.49 & 4.42 & 5.09 & 4.87 & 4.35 & 13.72 \\
\hline $10 / 19 / 93$ & $11 c$ & 0.00 & 5.22 & 4.65 & 4.27 & 4.79 & 5.00 & 4.51 & 13.97 \\
\hline $10 / 19 / 93$ & $11 \mathrm{~d}$ & 0.00 & 5.31 & 4.44 & 4.11 & 5.01 & 5.51 & 4.43 & 14.26 \\
\hline $10 / 19 / 93$ & $11 \mathrm{e}$ & 0.00 & 5.39 & 4.64 & 4.49 & 4.54 & 4.38 & 4.21 & 14.06 \\
\hline $10 / 19 / 93$ & $11 f$ & 0.00 & 5.24 & 4.51 & 4.45 & 4.93 & 4.61 & 4.23 & 14.38 \\
\hline $10 / 20 / 93$ & $11 \mathrm{~g}$ & 0.00 & 5.37 & 4.36 & 4.31 & 4.64 & 5.26 & 4.97 & 14.45 \\
\hline $10 / 20 / 93$ & $11 \mathrm{~h}$ & 0.00 & 5.34 & 4.63 & 4.58 & 5.17 & 5.19 & 4.31 & 14.14 \\
\hline $10 / 20 / 93$ & $11 i$ & 0.00 & 5.31 & 4.49 & 4.35 & 5.55 & 6.01 & 4.79 & 14.13 \\
\hline $10 / 21 / 93$ & $11 \mathrm{j}$ & 0.00 & 5.07 & 4.64 & 4.52 & 4.92 & 4.54 & 4.36 & 14.01 \\
\hline $10 / 21 / 93$ & $11 \mathrm{k}$ & 0.00 & 5.12 & 4.31 & 4.37 & 5.05 & 4.92 & 4.25 & 13.99 \\
\hline $10 / 21 / 93$ & 111 & 0.00 & 5.29 & 4.35 & 4.49 & 4.90 & 4.66 & 4.32 & 14.11 \\
\hline $10 / 21 / 93$ & $11 \mathrm{~m}$ & 0.00 & 5.21 & 4.69 & 4.22 & 5.31 & 5.24 & 4.32 & 14.64 \\
\hline $10 / 21 / 93$ & $11 n$ & 0.00 & 5.29 & 4.61 & 4.61 & 6.11 & 6.00 & 4.49 & 14.19 \\
\hline $10 / 22 / 93$ & $11 p$ & 0.00 & 5.25 & 4.37 & 4.44 & 5.23 & 5.16 & 4.39 & 14.47 \\
\hline $10 / 22 / 93$ & $11 q$ & 0.00 & 5.30 & 4.59 & 4.60 & 4.87 & 5.00 & 4.54 & 14.74 \\
\hline $10 / 22 / 93$ & $11 \mathrm{r}$ & 0.00 & 5.01 & 4.59 & 4.48 & 5.47 & 6.06 & 4.42 & 14.02 \\
\hline $10 / 22 / 93$ & $11 \mathrm{~s}$ & 0.00 & 5.06 & 4.33 & 4.65 & 5.90 & 6.56 & 4.64 & 13.94 \\
\hline $10 / 22 / 93$ & $11 \mathrm{t}$ & 0.00 & 5.15 & 4.59 & 4.46 & 4.83 & 4.73 & 4.32 & 14.48 \\
\hline $10 / 25 / 93$ & $11 u$ & 0.00 & 5.23 & 4.48 & 4.44 & 5.10 & 5.14 & 4.02 & 14.16 \\
\hline
\end{tabular}


Data Summary

\begin{tabular}{|c|c|c|c|c|c|c|c|c|c|}
\hline DATE & EXP & S1MH & S2MH & S3MH & S4MH & S5MH & S6MH & S7MH & MDOT \\
\hline $10 / 25 / 93$ & $11 \mathrm{v}$ & 0.00 & 5.42 & 4.43 & 4.66 & 4.88 & 5.05 & 4.65 & 14.62 \\
\hline $10 / 25 / 93$ & $11 w$ & 0.00 & 5.37 & 4.54 & 4.40 & 5.32 & 5.73 & 4.50 & 14.65 \\
\hline $10 / 25 / 93$ & $11 x$ & 0.00 & 5.07 & 4.37 & 4.69 & 6.98 & 6.02 & 4.79 & 14.91 \\
\hline $11 / 14 / 93$ & $10 x$ & 0.00 & 4.69 & 4.65 & 4.14 & 4.32 & 4.65 & 4.14 & 17.07 \\
\hline $11 / 21 / 93$ & 110 & 0.00 & 4.97 & 4.30 & 4.62 & 4.25 & 4.57 & 4.83 & 14.40 \\
\hline $2 / 23 / 94$ & & 0.00 & 0.00 & 0.00 & 0.00 & 0.00 & 0.00 & 0.00 & 19.18 \\
\hline $2 / 23 / 94$ & & 0.00 & 0.00 & 0.00 & 0.00 & 0.00 & 0.00 & 0.00 & \\
\hline $2 / 23 / 94$ & & 0.00 & 0.00 & 0.00 & 0.00 & 0.00 & 0.00 & 0.00 & \\
\hline $2 / 24 / 94$ & & 0.00 & 0.00 & 0.00 & 0.00 & 0.00 & 0.00 & 0.00 & 19.11 \\
\hline $4 / 18 / 94$ & $12 a$ & 0.00 & 0.00 & 9.00 & 0.00 & 0.00 & 0.00 & 0.00 & 0.00 \\
\hline $4 / 18 / 94$ & $12 b$ & 0.00 & 0.00 & 0.00 & 0.00 & 0.00 & 0.00 & 0.00 & 0.00 \\
\hline $4 / 18 / 94$ & $12 c$ & 0.00 & 0.00 & 0.00 & 0.00 & 0.00 & 0.00 & 0.00 & 0.00 \\
\hline $4 / 18 / 94$ & $12 \mathrm{~d}$ & 0.00 & 0.00 & 0.00 & 0.00 & 0.00 & 0.00 & 0.00 & 0.00 \\
\hline $4 / 18 / 94$ & $12 e$ & 0.00 & 0.00 & 0.00 & 0.00 & 0.00 & 0.00 & 0.00 & 0.00 \\
\hline $4 / 18 / 94$ & A12 & 0.00 & 0.00 & 0.00 & 0.00 & 0.00 & 0.00 & 0.00 & \\
\hline $4 / 21 / 94$ & $\mathrm{~B} 12$ & 0.00 & 0.00 & 0.00 & 0.00 & 0.00 & 0.00 & 0.00 & \\
\hline $5 / 2 / 94$ & $12 f$ & 0.00 & 0.00 & 0.00 & 0.00 & 0.00 & 0.00 & 0.00 & 0.00 \\
\hline $5 / 2 / 94$ & $12 \mathrm{~g}$ & 0.00 & 0.00 & 0.00 & 0.00 & 0.00 & 0.00 & 0.00 & 0.00 \\
\hline $5 / 2 / 94$ & $12 \mathrm{~h}$ & 0.00 & 0.00 & 0.00 & 0.00 & 0.00 & 0.00 & 0.00 & 0.00 \\
\hline $5 / 2 / 94$ & $12 i$ & 0.00 & 0.00 & 0.00 & 0.00 & 0.00 & 0.00 & 0.00 & 0.00 \\
\hline $5 / 2 / 94$ & $12 j$ & 0.00 & 9.00 & 8.33 & 6.86 & 7.07 & 7.10 & 5.24 & 1.34 \\
\hline $5 / 3 / 94$ & $12 r$ & 0.00 & 5.99 & 6.27 & 5.84 & 5.58 & 5.17 & 4.02 & 0.53 \\
\hline $5 / 3 / 94$ & $12 \mathrm{k}$ & 0.00 & 0.00 & 0.00 & 0.00 & 0.00 & 0.00 & 0.00 & 0.00 \\
\hline $5 / 3 / 94$ & 121 & 0.00 & 0.00 & 0.00 & 0.00 & 0.00 & 0.00 & 0.00 & 0.00 \\
\hline $5 / 3 / 94$ & $12 \mathrm{~m}$ & 0.00 & 0.00 & 0.00 & 0.00 & 0.00 & 0.00 & 0.00 & 0.00 \\
\hline $5 / 3 / 94$ & $12 n$ & 0.00 & 0.00 & 0.00 & 0.00 & 0.00 & 0.00 & 0.00 & 0.00 \\
\hline $5 / 3 / 94$ & 120 & 0.00 & 7.32 & 6.71 & 6.41 & 6.34 & 6.06 & 4.82 & 1.10 \\
\hline $5 / 3 / 94$ & $12 p$ & 0.00 & 0.00 & 0.00 & 0.00 & 0.00 & 0.00 & 0.00 & 0.00 \\
\hline $5 / 3 / 94$ & $12 q$ & 0.00 & 0.00 & 0.00 & 0.00 & 0.00 & 0.00 & 0.00 & 0.00 \\
\hline $5 / 3 / 94$ & $12 \mathrm{~s}$ & 0.00 & 6.96 & 6.69 & 6.47 & 6.36 & 6.06 & 4.73 & 2.62 \\
\hline $5 / 3 / 94$ & $12 t$ & 0.00 & 7.77 & 7.51 & 6.85 & 7.16 & 7.01 & 5.86 & 3.11 \\
\hline $5 / 5 / 94$ & $12 u$ & 0.00 & 0.00 & 0.00 & 0.00 & 0.00 & 0.00 & 0.00 & 0.00 \\
\hline $5 / 5 / 94$ & $12 v$ & 0.00 & 5.13 & 4.98 & 4.96 & 4.89 & 4.77 & 3.77 & 0.53 \\
\hline $5 / 6 / 94$ & $12 w$ & 0.00 & 4.62 & 4.25 & 4.09 & 4.14 & 4.27 & 3.96 & 4.64 \\
\hline $5 / 6 / 94$ & $12 x$ & 0.00 & 5.62 & 5.49 & 5.34 & 5.26 & 5.22 & 4.70 & 7.29 \\
\hline $5 / 6 / 94$ & $12 y$ & 0.00 & 9.12 & 7.37 & 6.63 & 6.26 & 6.08 & 5.47 & 13.23 \\
\hline $5 / 6 / 94$ & $12 z$ & 0.00 & 3.51 & 3.45 & 3.48 & 3.56 & 3.44 & 3.11 & 2.62 \\
\hline $5 / 6 / 94$ & $13 a$ & 0.00 & 4.52 & 4.39 & 4.42 & 4.34 & 4.47 & 4.31 & 5.42 \\
\hline $5 / 6 / 94$ & $13 b$ & 0.00 & 5.56 & 5.07 & 4.86 & 4.92 & 5.13 & 4.78 & 8.82 \\
\hline $5 / 6 / 94$ & $13 c$ & 0.00 & 9.32 & 7.22 & 6.29 & 6.20 & 6.01 & 5.91 & 16.74 \\
\hline $5 / 9 / 94$ & $13 d$ & 0.00 & 0.00 & 0.00 & 0.00 & 0.00 & 0.00 & 0.00 & 0.00 \\
\hline $5 / 9 / 94$ & $13 e$ & 0.00 & 3.19 & 2.86 & 2.66 & 3.03 & 3.03 & 2.92 & 3.20 \\
\hline $5 / 9 / 94$ & $13 \mathrm{f}$ & 0.00 & 4.07 & 3.78 & 3.64 & 3.60 & 3.60 & 3.60 & 7.62 \\
\hline $5 / 9 / 94$ & $13 \mathrm{~g}$ & 0.00 & 5.38 & 4.77 & 4.43 & 4.41 & 4.37 & 4.30 & 11.51 \\
\hline $5 / 9 / 94$ & $13 \mathrm{~h}$ & 0.00 & 9.24 & 7.36 & 6.42 & 6.50 & 6.09 & 5.92 & 24.43 \\
\hline $5 / 11 / 94$ & $13 i$ & 0.00 & 0.00 & 0.00 & 0.00 & 0.00 & 0.00 & 0.00 & 0.00 \\
\hline $5 / 11 / 94$ & $13 j$ & 0.00 & 2.80 & 2.59 & 2.36 & 2.28 & 2.72 & 2.55 & 4.95 \\
\hline $5 / 11 / 94$ & $13 k$ & 0.00 & 3.53 & 3.22 & 3.36 & 3.27 & 3.15 & 3.37 & 8.36 \\
\hline $5 / 11 / 94$ & $13 !$ & 0.00 & 4.82 & 4.31 & 3.81 & 3.91 & 4.05 & 4.28 & 13.74 \\
\hline $5 / 11 / 94$ & $13 \mathrm{~m}$ & 0.00 & 7.33 & 6.77 & 6.01 & 5.92 & 5.94 & 5.73 & 32.20 \\
\hline
\end{tabular}


Data Summary

\begin{tabular}{|c|c|c|c|c|c|c|c|c|c|}
\hline DATE & EXP & SIMH & S2MH & S3MH & S4MH & S5MH & S6MH & S7MH & MDOT \\
\hline $5 / 11 / 94$ & $13 n$ & 0.00 & 1.61 & 1.67 & 1.99 & 1.74 & 1.76 & 1.47 & 1.19 \\
\hline $5 / 11 / 94$ & 130 & 0.00 & 2.65 & 2.70 & 2.55 & 2.78 & 2.45 & 2.38 & 4.79 \\
\hline $5 / 11 / 94$ & $13 p$ & 0.00 & 3.57 & 3.61 & 3.14 & 3.53 & 3.22 & 3.82 & 9.26 \\
\hline $5 / 11 / 94$ & $13 q$ & 0.00 & 4.91 & 4.18 & 3.88 & 4.07 & 4.20 & 4.18 & 14.04 \\
\hline $5 / 11 / 94$ & $13 r$ & 0.00 & 7.17 & 6.41 & 5.90 & 5.84 & 5.74 & 5.61 & 30.96 \\
\hline $5 / 13 / 94$ & $13 s$ & 0.00 & 6.41 & 6.09 & 5.79 & 6.10 & 6.03 & 5.98 & 32.55 \\
\hline $5 / 13 / 94$ & $13 \mathrm{t}$ & 0.00 & 4.57 & 4.61 & 4.19 & 4.35 & 4.89 & 4.75 & 16.59 \\
\hline $5 / 13 / 94$ & $13 u$ & 0.00 & 3.56 & 3.74 & 3.56 & 3.71 & 3.68 & 3.57 & 10.71 \\
\hline $5 / 13 / 94$ & $13 v$ & 0.00 & 2.71 & 2.71 & 2.84 & 2.74 & 2.65 & 2.69 & 5.56 \\
\hline $5 / 13 / 94$ & $13 w$ & 0.00 & 1.42 & 1.45 & 1.29 & 1.33 & 1.28 & 1.18 & 1.05 \\
\hline $5 / 13 / 94$ & $13 x$ & 0.00 & 5.65 & 5.42 & 5.25 & 5.35 & 5.65 & 5.42 & 27.95 \\
\hline $5 / 13 / 94$ & $13 y$ & 0.00 & 4.37 & 4.31 & 4.10 & 4.35 & 4.58 & 3.91 & 15.89 \\
\hline $5 / 13 / 94$ & $13 z$ & 0.00 & 3.44 & 3.65 & 3.24 & 3.34 & 3.75 & 3.44 & 9.76 \\
\hline $5 / 16 / 94$ & $14 a$ & 00 & 2.55 & 2.58 & 2.41 & 2.42 & 2.57 & 2.53 & 4.73 \\
\hline $5 / 16 / 94$ & $14 b$ & 0.00 & 1.30 & 1.35 & 1.09 & 0.83 & 0.29 & 0.19 & 0.76 \\
\hline $5 / 16 / 94$ & $14 c$ & 0.00 & 5.59 & 5.56 & 5.40 & 5.53 & 5.84 & 5.84 & 29.00 \\
\hline $3 / 94$ & $14 d$ & 0.00 & 4.30 & 4.15 & 4.07 & 4.28 & 4.18 & 4.09 & 15.06 \\
\hline 194 & $14 \mathrm{e}$ & 0.00 & 3.34 & 3.50 & 3.26 & 3.39 & 3.39 & 3.32 & 9.28 \\
\hline 194 & $14 f$ & .00 & 2.47 & 2.45 & 2.26 & 2.34 & 2.31 & 2.33 & 4.78 \\
\hline $5 / 17 / 94$ & $14 \mathrm{~g}$ & 0.00 & 0.75 & 0.17 & 0.17 & 0.15 & 0.15 & 1.44 & 0.70 \\
\hline $5 / 17 / 94$ & $14 \mathrm{~h}$ & 0.00 & 5.26 & 5.40 & 4.93 & 5.27 & 5.36 & 5.26 & 26.77 \\
\hline $5 / 17 / 94$ & $14 i$ & 0.00 & 4.20 & 4.17 & 3.79 & 3.87 & 3.73 & 3.58 & 16.69 \\
\hline $5 / 17 / 94$ & $14 j$ & 0.00 & 3.30 & 3.22 & 2.68 & 2.54 & 2.24 & 1.95 & 10.79 \\
\hline $5 / 17 / 94$ & $14 k$ & 00 & 2.19 & 1.70 & 1.19 & 0.97 & 0.86 & 0.80 & 5.49 \\
\hline $5 / 17 / 94$ & $14 !$ & 00 & 0.67 & 0.35 & 0.27 & 0.24 & 0.22 & 0.21 & 1.22 \\
\hline $5 / 17 / 94$ & $14 \mathrm{~m}$ & 00 & 4.93 & 5.42 & 4.89 & 4.98 & 4.89 & 4.95 & 25.23 \\
\hline $5 / 20 / 94$ & $14 n$ & 00 & 4.35 & 4.33 & 4.02 & 3.83 & 3.92 & 4.22 & 15.79 \\
\hline $5 / 20 / 94$ & 140 & 0.00 & 3.23 & 3.40 & 3.17 & 3.20 & 3.30 & 3.41 & 9.62 \\
\hline $5 / 20 / 94$ & $14 p$ & 0.00 & 2.44 & 2.45 & 2.28 & 2.25 & 2.24 & 2.31 & 4.81 \\
\hline $5 / 20 / 94$ & $14 q$ & 0.00 & 1.35 & 1.55 & 1.42 & 1.19 & 1.62 & 1.34 & 0.92 \\
\hline $5 / 20 / 94$ & $14 r$ & 0.00 & 5.20 & 5.25 & 4.89 & 5.31 & 5.52 & 5.35 & 27.98 \\
\hline $5 / 20 / 94$ & $14 \mathrm{~s}$ & 0.00 & 4.31 & 4.22 & 3.94 & 4.00 & 3.99 & 3.96 & 16.46 \\
\hline $5 / 20 / 94$ & $14 t$ & 00 & 3.34 & 3.25 & 3.00 & 2.87 & 2.71 & 2.60 & 10.49 \\
\hline $5 / 23 / 94$ & $14 u$ & & 0.44 & 0.25 & 0.20 & 0.19 & 0.16 & 0.15 & 1.24 \\
\hline 194 & $14 v$ & & 5.18 & 5.18 & 4.88 & 01 & 4.89 & 4.77 & 27.11 \\
\hline $5 / 23 / 94$ & $14 w$ & 0.00 & 3.07 & 3.32 & 3.60 & 3.68 & 3.92 & 4.25 & 17.31 \\
\hline $5 / 23 / 94$ & $14 x$ & 0.00 & 3.30 & 2.84 & 2.40 & 2.10 & 1.79 & 1.56 & 10.83 \\
\hline $5 / 23 / 94$ & $14 y$ & 0.00 & 1.97 & 1.38 & 1.00 & 0.87 & 0.80 & 0.73 & 5.63 \\
\hline $5 / 23 / 94$ & $14 z$ & 0.00 & 0.35 & 0.23 & 0.17 & 0.16 & 0.15 & 0.13 & 1.27 \\
\hline $5 / 27 / 94$ & $15 a$ & 0.00 & 5.08 & 4.75 & 4.26 & 3.98 & 3.67 & 3.38 & 29.31 \\
\hline $5 / 27 / 94$ & $15 b$ & 0.00 & 3.95 & 3.44 & 2.82 & 2.42 & 2.15 & 1.95 & 17.87 \\
\hline $5 / 30 / 94$ & $15 \mathrm{c}$ & 00 & 2.71 & 1.98 & 1.54 & 1.33 & 1.18 & 1.10 & 10.34 \\
\hline $5 / 30 / 94$ & & & 1.33 & 0.90 & 0.72 & 0.64 & 0.60 & 0.56 & 5.29 \\
\hline $5 / 30 / 94$ & $15 e$ & & 0.25 & 0.17 & 0.13 & 0.12 & 0.10 & 0.10 & 1.10 \\
\hline $5 / 30 / 94$ & $15 f$ & & 4.40 & 3.92 & 3.35 & 3.05 & 2.75 & 2.53 & 25.98 \\
\hline $5 / 30 / 94$ & $15 q$ & 0.00 & 3.87 & 3.12 & 2.48 & 2.16 & 1.91 & 1.75 & 18.34 \\
\hline $5 / 30 / 94$ & $15 \mathrm{~h}$ & 0.00 & 2.70 & 1.99 & 1.58 & 1.38 & 1.26 & 1.18 & 12.05 \\
\hline $5 / 30 / 94$ & $15 i$ & 0.00 & 1.40 & 0.99 & 0.82 & 0.73 & 0.68 & 0.64 & 6.10 \\
\hline & $17 d$ & 0.00 & 0.00 & 0.00 & 0.00 & 0.00 & 0.00 & 0.00 & \\
\hline $8 / 16 / 94$ & $17 e$ & 0.00 & 0.00 & 0.00 & 0.00 & 0.00 & 0.00 & 0.00 & \\
\hline
\end{tabular}




\begin{tabular}{|c|c|c|c|c|c|c|c|c|c|}
\hline DATE & EXP & S1MH & S2MH & S3MH & S4MH & S5MH & S6MH & S7MH & MDOT \\
\hline $8 / 17 / 94$ & $17 f$ & 0.00 & 0.00 & 0.00 & 0.00 & 0.00 & 0.00 & 0.00 & \\
\hline $8 / 17 / 94$ & $17 g$ & 0.00 & 0.00 & 0.00 & 0.00 & 0.00 & 0.00 & 0.00 & \\
\hline $8 / 18 / 94$ & $17 \mathrm{~h}$ & 0.00 & 0.34 & 0.27 & 0.21 & 0.20 & 0.19 & 0.19 & 1.55 \\
\hline $8 / 18 / 94$ & $17 j$ & 0.00 & 0.00 & 0.00 & 0.00 & 0.00 & 0.00 & 0.00 & \\
\hline $8 / 18 / 94$ & $17 k$ & 0.00 & 0.00 & 0.00 & 0.00 & 0.00 & 0.00 & 0.00 & \\
\hline $9 / 8 / 94$ & 171 & 0.00 & 0.00 & 0.00 & 0.00 & 0.00 & 0.00 & 0.00 & \\
\hline $9 / 9 / 94$ & $17 \mathrm{~m}$ & 0.00 & 4.41 & 4.50 & 4.28 & 4.43 & 4.39 & 4.38 & 18.65 \\
\hline $9 / 12 / 94$ & $17 n$ & 0.00 & 4.36 & 4.40 & 4.31 & 4.31 & 4.29 & 4.31 & 18.82 \\
\hline $9 / 12 / 94$ & 170 & 0.00 & 4.34 & 4.30 & 4.19 & 4.31 & 4.34 & 4.44 & 17.59 \\
\hline $9 / 12 / 94$ & $17 p$ & 0.00 & 4.30 & 4.35 & 4.24 & 4.22 & 0.00 & 4.37 & 19.16 \\
\hline $9 / 12 / 94$ & $17 q$ & 0.00 & 4.43 & 4.44 & 4.27 & 4.36 & 4.38 & 4.46 & 19.19 \\
\hline $9 / 13 / 94$ & $17 r$ & 0.00 & 4.30 & 4.37 & 4.23 & 4.25 & 4.44 & 4.32 & 18.97 \\
\hline $9 / 13 / 94$ & $17 \mathrm{~s}$ & 0.00 & 4.24 & 4.39 & 4.19 & 4.25 & 4.29 & 4.25 & 18.67 \\
\hline $9 / 13 / 94$ & $17 t$ & 0.00 & 4.26 & 4.30 & 4.26 & 4.31 & 4.27 & 4.33 & 18.07 \\
\hline $9 / 14 / 94$ & $17 u$ & 0.00 & 4.18 & 4.32 & 4.21 & 4.19 & 4.25 & 4.20 & 19.24 \\
\hline $9 / 14 / 94$ & $17 v$ & 0.00 & 4.20 & 4.23 & 4.17 & 4.18 & 0.00 & 4.20 & 20.35 \\
\hline $9 / 14 / 94$ & $17 w$ & 0.00 & 4.13 & 4.17 & 4.09 & 4.10 & 4.04 & 4.16 & 18.75 \\
\hline $9 / 14 / 94$ & $17 x$ & 0.00 & 3.84 & 3.89 & 3.68 & 3.80 & 3.79 & 3.79 & 19.55 \\
\hline $9 / 14 / 94$ & $17 y$ & 0.00 & 3.71 & 3.63 & 3.56 & 3.55 & 3.48 & 3.54 & 18.29 \\
\hline $9 / 14 / 94$ & 172 & 0.00 & 3.92 & 3.94 & 3.83 & 3.86 & 3.83 & 3.93 & 18.94 \\
\hline $9 / 14 / 94$ & $18 a$ & 0.00 & 3.67 & 3.61 & 3.56 & 3.54 & 3.49 & 3.54 & 17.71 \\
\hline $9 / 14 / 94$ & $18 b$ & 0.00 & 3.74 & 3.73 & 3.66 & 3.69 & 3.67 & 3.76 & 17.95 \\
\hline $9 / 14 / 94$ & $18 \mathrm{c}$ & 0.00 & 3.65 & 3.61 & 3.49 & 3.53 & 3.46 & 3.51 & 18.14 \\
\hline $9 / 14 / 94$ & $18 d$ & 0.00 & 3.38 & 3.38 & 3.43 & 3.42 & 3.59 & 3.66 & 36.28 \\
\hline $9 / 25 / 94$ & & 0.00 & 0.00 & 0.00 & 0.00 & 0.00 & 0.00 & 0.00 & \\
\hline $10 / 6 / 94$ & $18 \mathrm{k}$ & 0.00 & 0.00 & 0.00 & 0.00 & 0.00 & 0.00 & 0.00 & \\
\hline $10 / 6 / 94$ & 181 & 0.00 & 0.00 & 0.00 & 0.00 & 0.00 & 0.00 & 0.00 & \\
\hline $10 / 7 / 94$ & $18 \mathrm{~m}$ & 0.00 & 0.00 & 0.00 & 0.00 & 0.00 & 0.00 & 0.00 & \\
\hline $10 / 7 / 94$ & $18 n$ & 0.00 & 0.00 & 0.00 & 0.00 & 0.00 & 0.00 & 0.00 & \\
\hline $10 / 10 / 94$ & 180 & 0.00 & 0.00 & 0.00 & 0.00 & 0.00 & 0.00 & 0.00 & \\
\hline $10 / 10 / 94$ & $18 p$ & 0.00 & 0.00 & 0.00 & 0.00 & 0.00 & 0.00 & 0.00 & \\
\hline $10 / 10 / 94$ & & 0.00 & 5.36 & 4.97 & 4.68 & 4.72 & 4.33 & 4.61 & 28.99 \\
\hline $10 / 10 / 94$ & & 0.00 & 8.52 & 7.81 & 7.02 & 6.65 & 6.00 & 4.82 & 3.02 \\
\hline $10 / 11 / 94$ & & 0.00 & 6.79 & 7.09 & 6.99 & 7.39 & 7.41 & 6.92 & 16.53 \\
\hline $10 / 14 / 94$ & $18 q$ & 0.00 & 0.00 & 0.00 & 0.00 & 0.00 & 0.00 & 0.00 & \\
\hline $10 / 25 / 94$ & $18 \mathrm{~s}$ & 0.00 & 0.00 & 0.00 & 0.00 & 0.00 & 0.00 & 0.00 & \\
\hline $10 / 26 / 94$ & $18 t$ & 0.00 & 0.00 & 0.00 & 0.00 & 0.00 & 0.00 & 0.00 & \\
\hline
\end{tabular}

